\title{
WestVirginiaUniversity
}

THE RESEARCH REPOSITORY @ WVU

Graduate Theses, Dissertations, and Problem Reports

2015

\section{Short-Term Resource Allocation and Management}

Denis da Cruz Pinha

Follow this and additional works at: https://researchrepository.wvu.edu/etd

\section{Recommended Citation}

Pinha, Denis da Cruz, "Short-Term Resource Allocation and Management" (2015). Graduate Theses,

Dissertations, and Problem Reports. 6422.

https://researchrepository.wvu.edu/etd/6422

This Dissertation is protected by copyright and/or related rights. It has been brought to you by the The Research Repository @ WVU with permission from the rights-holder(s). You are free to use this Dissertation in any way that is permitted by the copyright and related rights legislation that applies to your use. For other uses you must obtain permission from the rights-holder(s) directly, unless additional rights are indicated by a Creative Commons license in the record and/ or on the work itself. This Dissertation has been accepted for inclusion in WVU Graduate Theses, Dissertations, and Problem Reports collection by an authorized administrator of The Research Repository @ WVU.

For more information, please contact researchrepository@mail.wvu.edu. 


\title{
Short-Term Resource Allocation and Management
}

\author{
Denis da Cruz Pinha
}

\author{
Dissertation submitted to the \\ Statler College of Engineering and Mineral Resources at \\ West Virginia University \\ in partial fulfillment of the requirements for the degree of
}

Doctor of Philosophy

in

Industrial Engineering
Rashpal Ahluwalia, Ph.D., Chair
Fei Dai, Ph.D.
V. Jagannathan, Ph.D
Majid Jaridi, Ph.D.
Feng Yang, Ph.D.
Department of Industrial and Management Systems Engineering
Morgantown, West Virginia
2015

Keywords: Resource Management, Resource Constrained Project Scheduling, Project Management, Short-Term, Discrete Event Simulation, Decision Support System

Copyright 2015 Denis da Cruz Pinha 


\section{Abstract Short-Term Resource Allocation and Management}

\section{Denis da Cruz Pinha}

Almost all sectors of the economy, such as, government, healthcare, education, ship repair, construction, and manufacturing require project management. A key component of project management deals with scheduling of tasks such that limited resources are utilized in an effective manner. Current research on resource constrained project-scheduling has been classified as: a) Single project with single mode for various tasks, b) Single project with multiple task modes, c) Multiple projects with single task mode, and d) Multiple projects with multiple task modes.

This work extends the current multi-project, multi-mode scheduling techniques. The resources can be renewable, and non-renewable. In addition, it focuses on short term scheduling, that is, scheduling on an hourly, daily, or weekly basis. Long term scheduling assumes a stable system, that is, resources, priorities, and other constraints do no change during the scheduling period. In this research, short term scheduling assumes a dynamic system, that is, resources, priorities, and other constraints change over time.

A hybrid approach is proposed to address the dynamic nature of the problem. It is based on discrete event simulation and a set of empirical rules provided by the project manager. The project manager is assumed to be highly knowledgeable about the project. He/she is regarded as an integral part of the system. Such an approach is better suited to deal with "real world" scheduling. The proposed approach does not seek to provide a single "optimum" solution, instead, it generates a series of feasible solutions, along with the impact of each solution on schedule and cost.

Two project case studies dealing with finding an optimum solution were selected from the literature. The proposed technique was applied to the data set in these studies. In both cases the proposed approach found the "optimum" solution. The model was then applied to two additional problems to test the features that could not be tested on the dataset from the literature.

As for practical implications, the proposed approach enhances the decision making process, by providing more resource allocation flexibility, and results in improved solutions in terms of total project duration and cost. From an academic viewpoint, this research enriches the existing literature, as it provides an extension of the resource constrained project scheduling problems, a discrete event simulation and four cases studies which highlights relevant issues to model properly the complexity of real-life projects. 


\section{Acknowledgements}

I would like to express my deepest gratitude to my advisor, Dr. Rashpal Ahluwalia or Dr. A, as I have kindly called him for 4 years. Dr. A supported me through good and tough times during my research period. His experience, his positive attitude for life, his financial support and his valuable advices helped me to overcome several research and life challenges. Dr.A's role was far beyond what I ever expected of an advisor. His guidance, his knowledge, and his kindness fulfilled a perfect combination for me. I finish this important step in my life to start a new one having Dr.A as an inspiration, as a good friend, and also knowing that several opportunities still there for us to work together. I feel so glad to have had Dr. A in my life.

Besides my advisor, I would like to thank my dissertation committee: Dr. Jaridi, Dr. Yang, Dr. Dai and Dr. Juggy for their insightful comments and encouragement, but also for the questions which improved my research from various perspectives. It is an honor for me to have you as my committee members. Special feeling of gratitude to Ms. Marie and Mr. Howard who have helped me infinite times during my time at West Virginia University.

I also would like to express my gratitude to Dr. Nimbarte, Dr. Currie, Dr. Creese, and Dr. Iskander for providing me a chance to work as Teaching Assistant with Dr. A and also for providing a friendly research environment in the Department of Industrial and Management System Engineering at West Virginia University. 


\section{Dedication}

I dedicate my dissertation to my wife, Carolina, and my son, Tiago. I would never have been able to finish my dissertation without their help. So much love!

A special feeling of gratitude to my loving parents, Cesar and Vera. My brother Daniel, his wife Paula, Vinicius, and Bernardo who have helped me infinite times. I also would like to dedicate this dissertation to Tia Helena and Tio Jose Lourenco who have supported me with encouragement words and optimism in tough times. My sister Andreia, brother Cesar, nephew Victor, my cousin Renato and my father in law Oscar who have never left my side, in spite of the distance. They have given me so much support and encouragement to finish my dissertation. I also dedicate this dissertation to my good friends Junior, Ana Candida, Bernardo, and little one Eduardo who have supported me throughout the process. I will always appreciate all they have done.

Special gratitude to Dr. Carvalho (Andrea) and Dr. Saisse (Manoel). They provided me several insights which improved my dissertation. I would also like to express my special feelings to Dr. Queiroz (Max) and Dr. Cury (Jose) of the Department of Automation and Systems Engineering at Federal University of Santa Catarina. They sparked my interest to pursue a higher education level and they are good friends.

I would like finally to thank God for supporting me spiritually throughout writing this dissertation and my life. 


\section{Table of Contents}

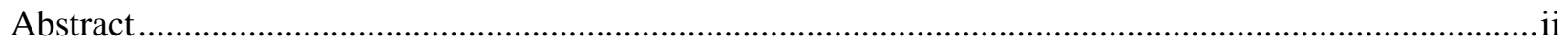

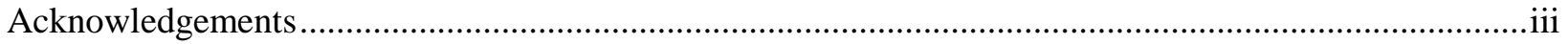

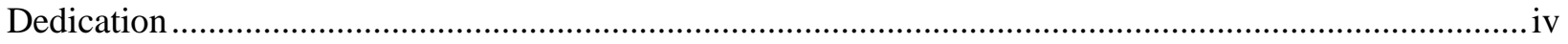

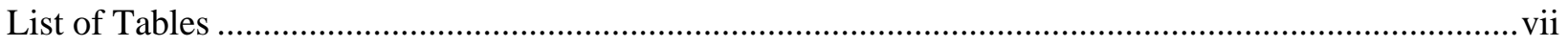

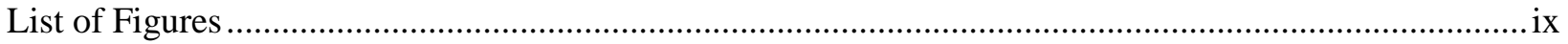

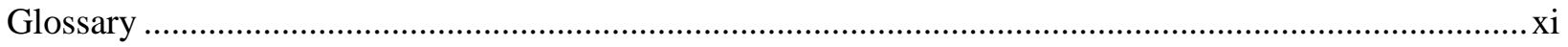

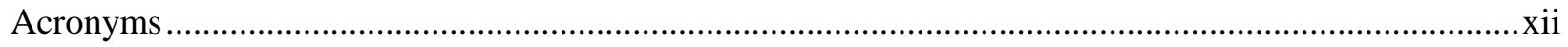

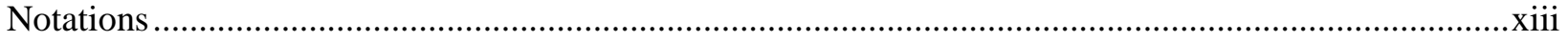

Chapter 1: Introduction and Literature Review .............................................................................. 1

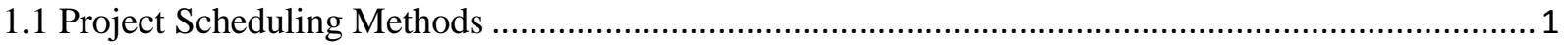

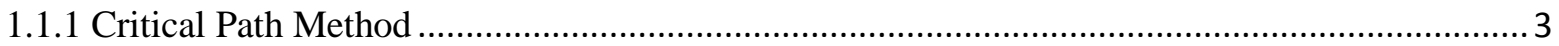

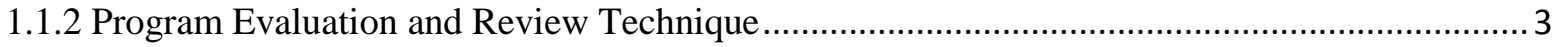

1.1.3 Resource Constrained Project Scheduling Problem (RCPSP) ............................................... 4

1.1.4 Multi-Mode Resource Constrained Project Scheduling Problem (MRCPSP) ........................... 6

1.1.5 Resource Constrained Multi-Project Scheduling Problem (RCMPSP) ................................... 7

1.1.6 Resource Constrained Project Scheduling Problem with Flexible Resource Profile (FRCPSP) 8

1.1.7 Multi-mode Resource Constrained Multi-Project Scheduling Problem (MRCMPSP)...............9

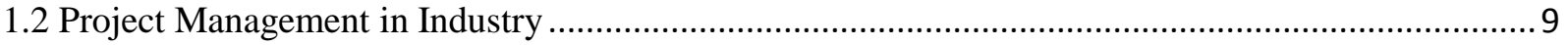

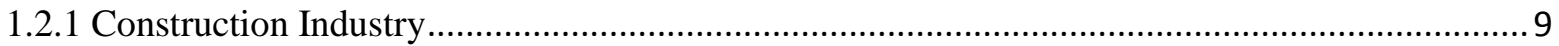

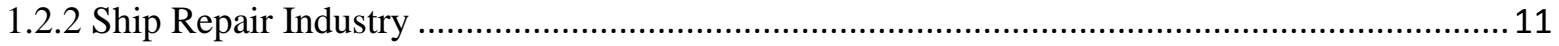

1.2.3 Limitations of the Commonly Used Tools ............................................................................ 13

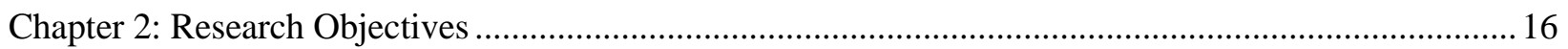

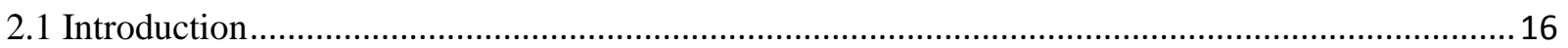

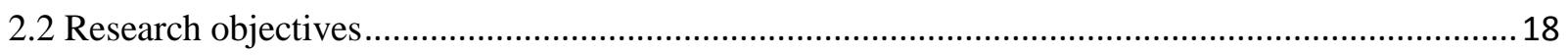

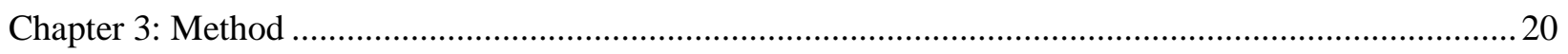

3.1 The Combinatorial Multi-Mode Resource Constrained Multi-Project Scheduling Problem............20

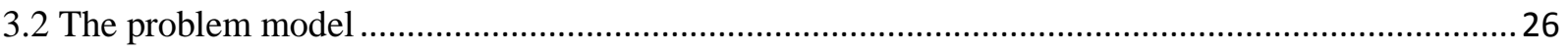

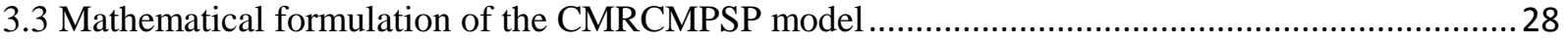

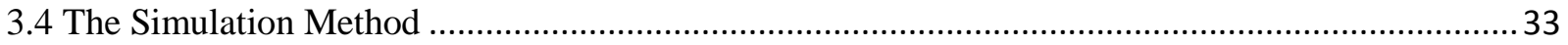

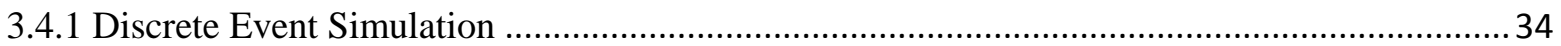

3.4.2 Multi-mode Scheduling and Queue Management.................................................................. 37

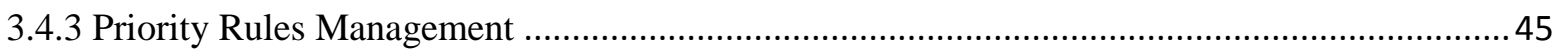

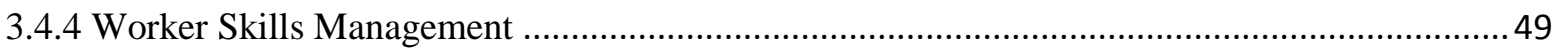




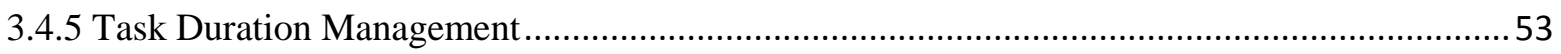

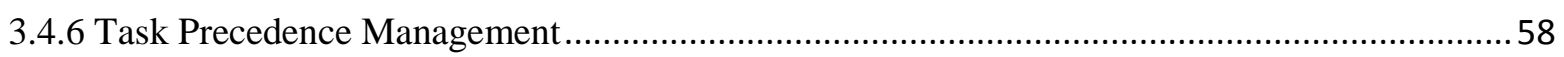

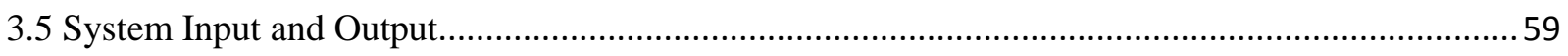

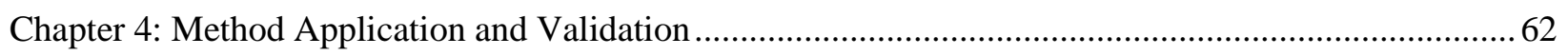

4.1 Case study 1: Construction of a Single Cell Box Culvert...........................................................62

4.2 Case study 2: Oil Refinery Maintenance ................................................................................... 79

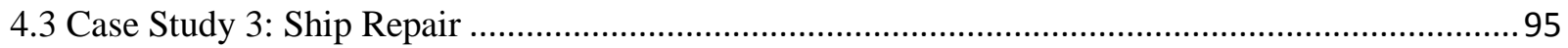

4.4 Case Study 4: Supply Chain Management of Motorcycle Assembly Line..................................104

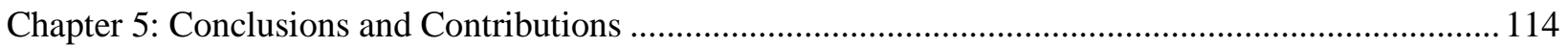

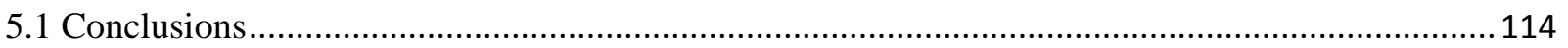

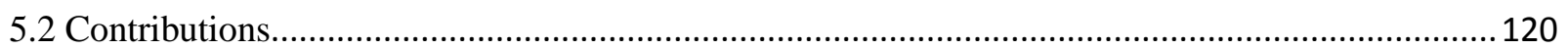

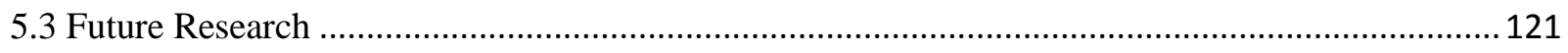

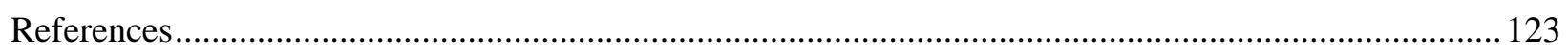

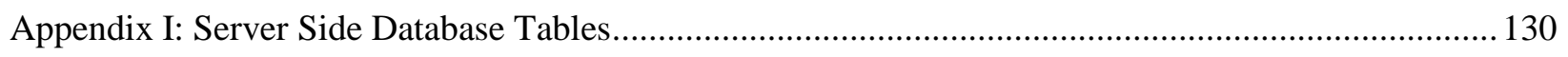

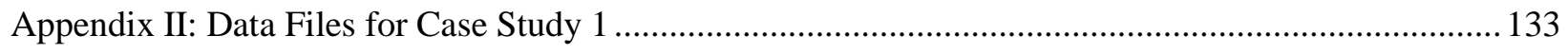

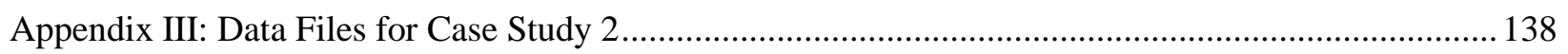

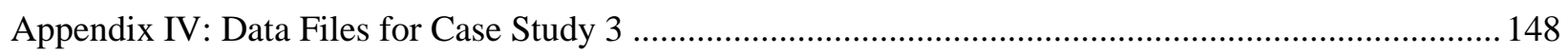




\section{List of Tables}

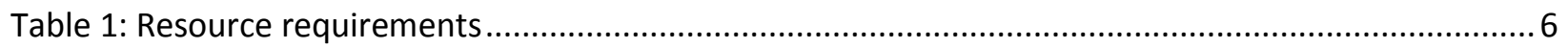

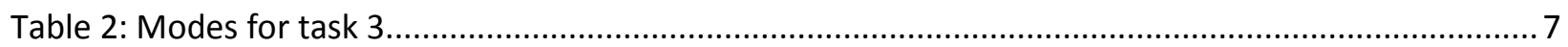

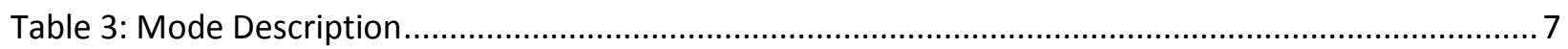

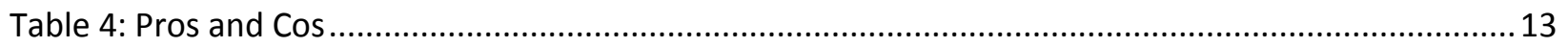

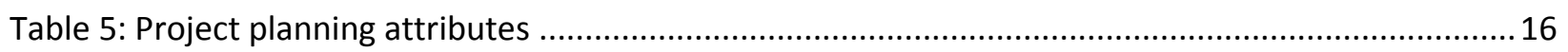

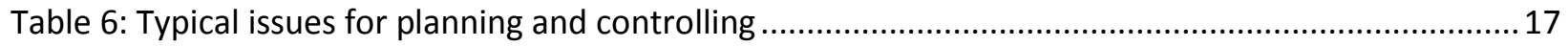

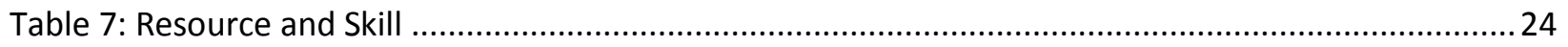

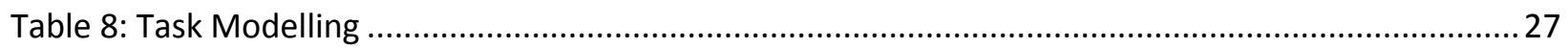

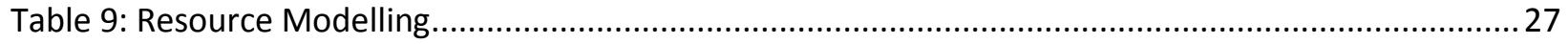

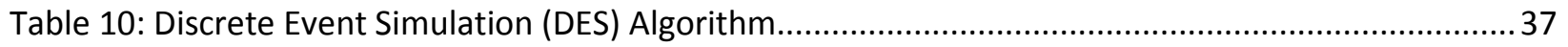

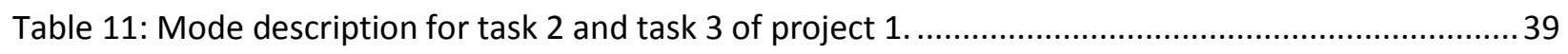

Table 12: Multi-mode Scheduling and Queue Management (MSQM) ..................................................4

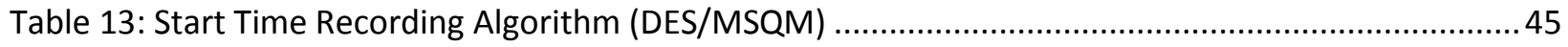

Table 14: Priority Rules Management (PRM) Algorithm.................................................................... 48

Table 15: Available Resource and Skills Management (ARSM) algorithm/WSM ...................................51

Table 16: Resource and Skills Requirement Management (RSRM) algorithm/WSM .............................52

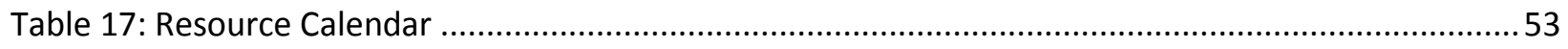

Table 18: Work Day Classification for Resource R1 on Monday.........................................................5

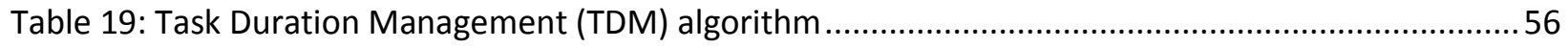

Table 20: Availability Verification Management (AVM) algorithm .......................................................5

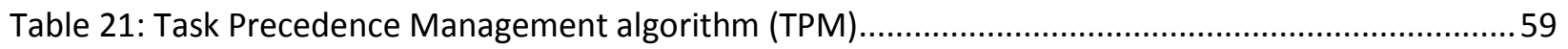

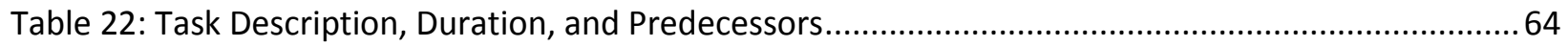

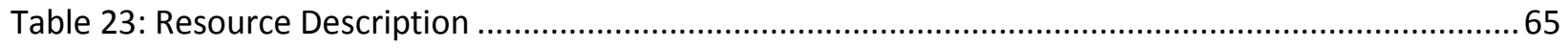

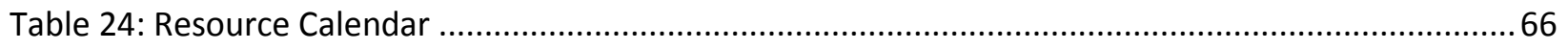

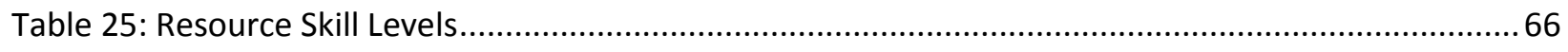

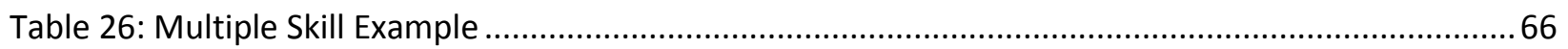

Table 27: Total Project Duration and Resource Utilization by STREAM for Scenario 1 and 2 ...................68

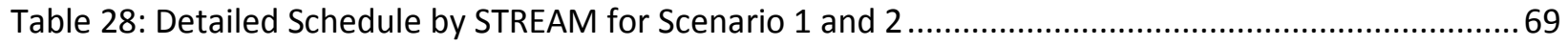

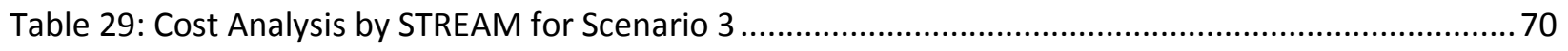

Table 30: Total Resource Duration and Resource Utilization by STREAM for Scenario 3 ........................71

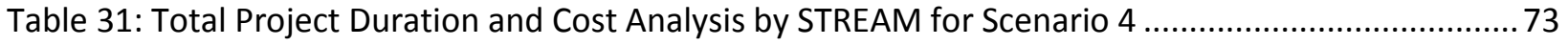

Table 32: Project Duration and Cost Analysis by STREAM for Scenario 5 ...............................................74

Table 33: Project Duration and Cost Analysis by STREAM for Scenario 6 ................................................. 75

Table 34: Task waiting for resources in scenario 7 (by STREAM) .....................................................76

Table 35: Total Project Duration and Total Cost for the Seven Scenarios ................................................78

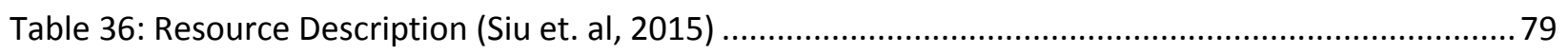

Table 37: Optimal total project duration and resource requirements (Siu et. al, 2015) ......................... 81

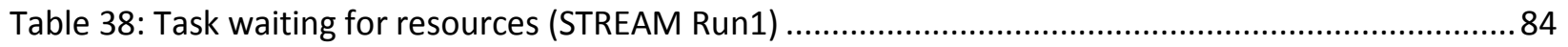

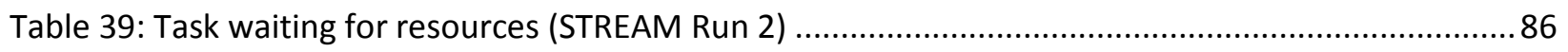

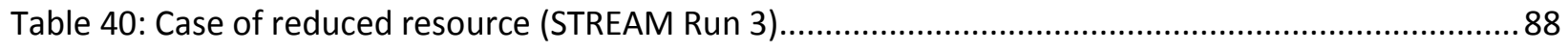

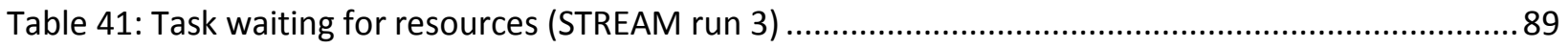




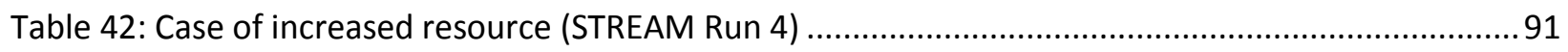

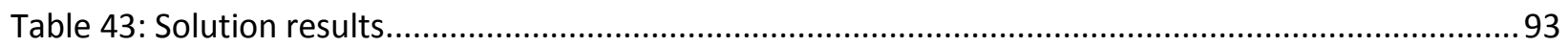

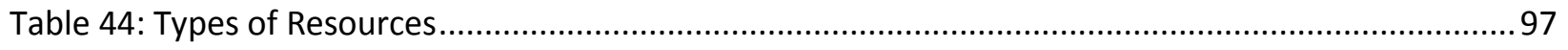

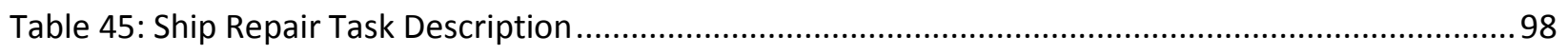

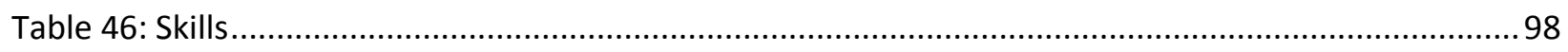

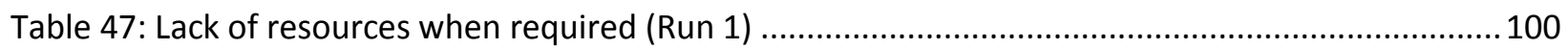

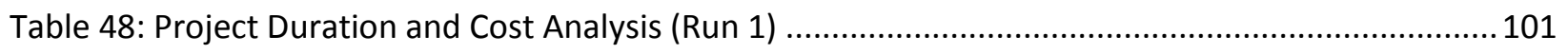

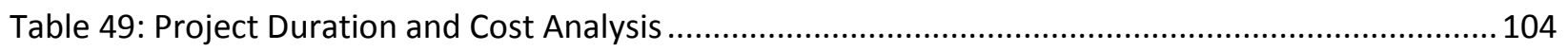

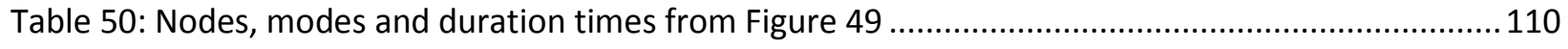

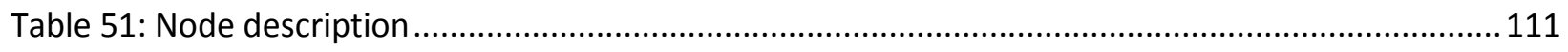

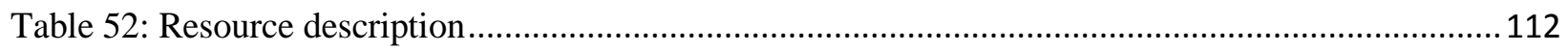




\section{List of Figures}

Figure 1: Task on Node Diagram for the Critical Path Method........................................................... 3

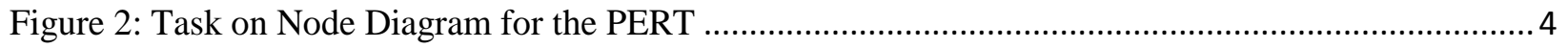

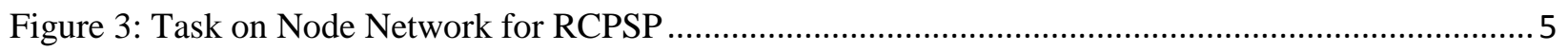

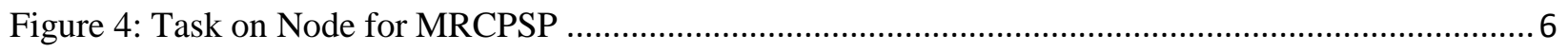

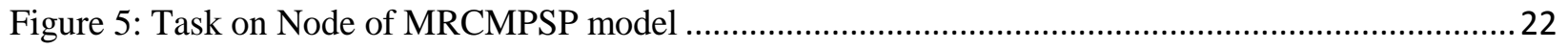

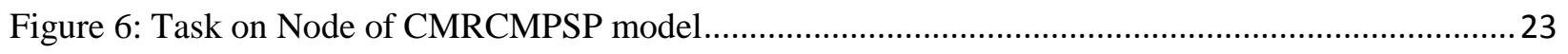

Figure 7: Task on node representation for multiple projects ..........................................................2

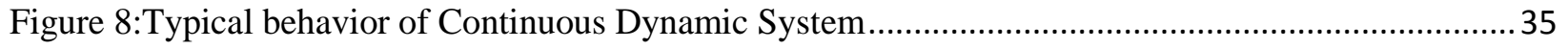

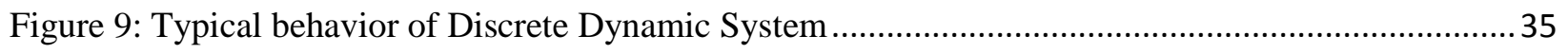

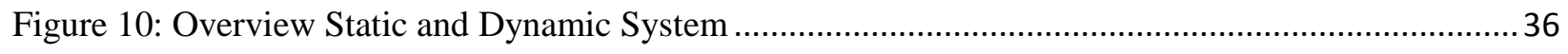

Figure 11: Selection of a queue to join and selection of a queue to serve ........................................... 38

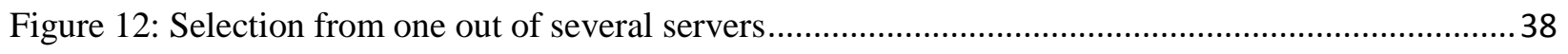

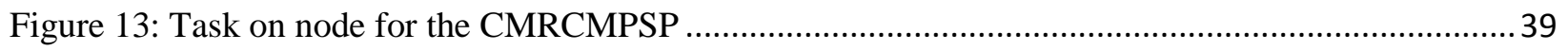

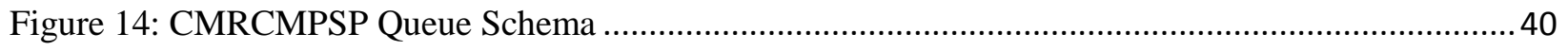

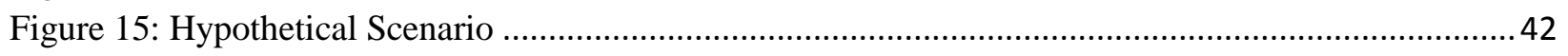

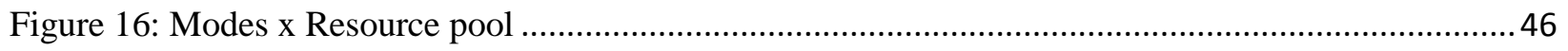

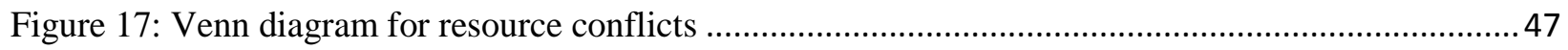

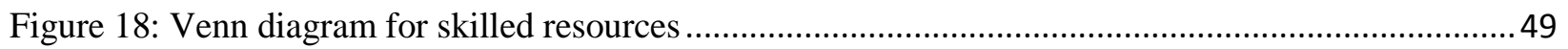

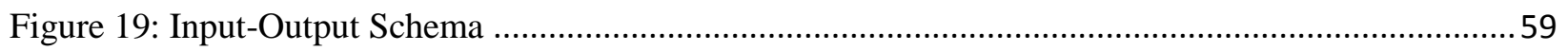

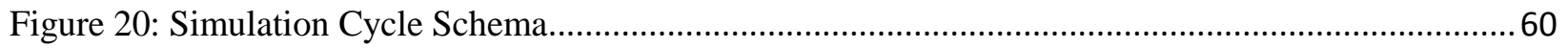

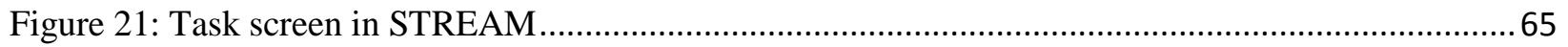

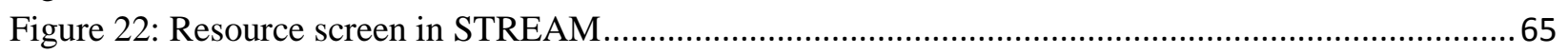

Figure 23: Resource Utilization Screen in STREAM ..................................................................... 70

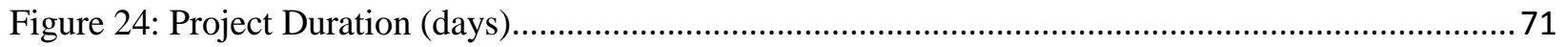

Figure 25: Project Cost (million USD) ……............................................................................... 71

Figure 26: Project Cost (million USD) for Skilled Labour between 15 and 38 ......................................72

Figure 27: Project Duration (days) for Four Simulation Runs............................................................ 73

Figure 28: Project Cost (million USD) for Four Simulation Runs .......................................................73

Figure 29: Task/Mode/Resources screen in STREAM .................................................................... 74

Figure 30: Project Duration (days) for Seven Scenarios........................................................................ 78

Figure 31: Project Cost (Millions USD) for Seven Scenarios ............................................................ 78

Figure 32: Task finish times for Siu et al., 2015 and STREAM Run 1 ................................................ 85

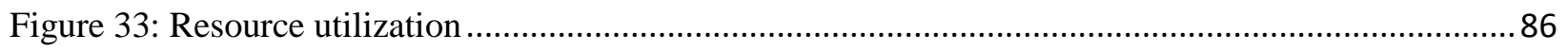

Figure 34: Task finish times for Siu et al., 2015 and STREAM Run 2 ................................................ 87

Figure 35: Task finish times for Siu et al., 2015 and STREAM Run 3 ...............................................90

Figure 36: Task finish times for Siu et al., 2015 and STREAM Run 4 ................................................92

Figure 37: Resource requirements for Siu et al., 2015, STREAM Run 1, STREAM Run 2, and STREAM

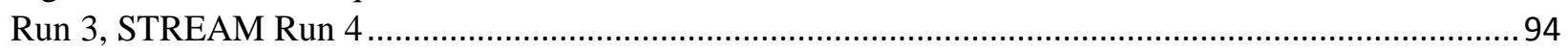

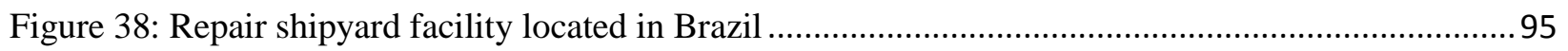

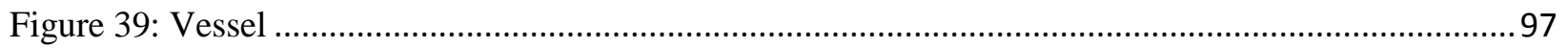

Figure 40: Multiple skills for a given resource in STREAM..............................................................99

Figure 41: Resource Utilization (Run 1) ................................................................................. 100

Figure 42: Accumulated Cost with nonrenewable issue in Task 61 ...................................................101 
Figure 43: Deviation of Due Dates with nonrenewable resource issue ..............................................102

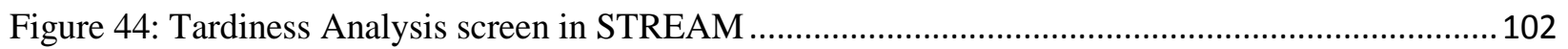

Figure 45: Accumulated cost with earlier deliver date for the nonrenewable resource ........................... 103

Figure 46: Deviation of Due Dates with Nonrenewable resource issue solved .....................................103

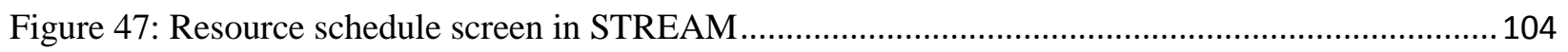

Figure 48: Supply chain structure from Ivanov et al (2014) .............................................................. 105

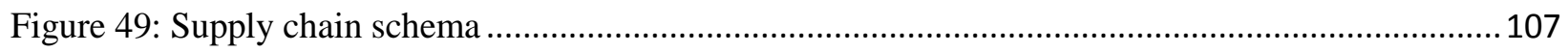

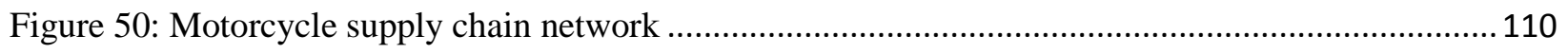

Figure 51: Gantt chart in STREAM for case study 4...................................................................... 113 


\section{Glossary}

\begin{tabular}{|c|c|}
\hline Term & Description \\
\hline Task & $\begin{array}{l}\text { Lowest level of work element (it cannot be divided further), that influences } \\
\text { project cost, duration, or resource utilization. }\end{array}$ \\
\hline Mode & Alternate ways of accomplishing the same task \\
\hline $\begin{array}{l}\text { Non-renewable } \\
\text { Resource }\end{array}$ & $\begin{array}{l}\text { Nonrenewable resource is a resource that is consumed when a task is carried out, } \\
\text { e.g. raw material, power, fuel, etc. }\end{array}$ \\
\hline Renewable Resources & Renewable resource are not consumed, e.g. facilities, equipment, workers, etc. \\
\hline $\begin{array}{l}\text { Doubly Constrained } \\
\text { Resource }\end{array}$ & $\begin{array}{l}\text { Doubly-constrained resources are a combination of the two previous categories } \\
\text { and are constrained per period. }\end{array}$ \\
\hline $\begin{array}{l}\text { Regular Measures of } \\
\text { Performance }\end{array}$ & $\begin{array}{l}\text { The minimization of the project duration is certainly the most common regular } \\
\text { measure of performance. Also, the minimization of lateness. }\end{array}$ \\
\hline $\begin{array}{l}\text { Non-regular } \\
\text { Measures of } \\
\text { Performance }\end{array}$ & $\begin{array}{l}\text { Non-regular measures of performance are those in which the regular measure } \\
\text { definition does not apply. This measure introduces financial aspects such as cost, } \\
\text { penalty costs, and quality cost. }\end{array}$ \\
\hline
\end{tabular}




\section{Acronyms}

\begin{tabular}{|l|l|}
\hline \multicolumn{1}{|c|}{ Acronyms } & \multicolumn{1}{c|}{ Description } \\
\hline RCPSP & Resource-Constrained Project Scheduling Problem \\
\hline MRCPSP & Multi-Mode Resource Constrained Project Scheduling Problem \\
\hline FRCPSP & RCPSP with Flexible resource profiles \\
\hline RCMPSP & Resource-Constrained Multi-Project Scheduling Problem \\
\hline MRCMPSP & Multi-mode Resource-Constrained Multi-Project Scheduling Problem \\
\hline CMRCMPSP & Combinatorial Multi-mode Resource-Constrained Multi-Project Scheduling Problem \\
\hline SSGS & Serial schedule generation scheme \\
\hline PSGS & Parallel schedule generation scheme \\
\hline PMSGS & Parallel mode schedule generation scheme \\
\hline ToN & Task-on-Node \\
\hline PERT & Program Evaluation Review Technique \\
\hline CPM & Critical Path Method \\
\hline JIT & Just-in-Time \\
\hline OPT & Optimized Production Technology \\
\hline
\end{tabular}




\section{Notations}

\begin{tabular}{|c|c|}
\hline Scalar & Description \\
\hline NP & Number of projects \\
\hline NS & Number of skills \\
\hline $\mathrm{NA}_{\mathrm{i}}$ & Number of tasks of project $\mathrm{i}$ \\
\hline $\mathrm{NM}_{\mathrm{i}, \mathrm{j}}$ & Number of modes for project $\mathrm{i}$, task $\mathrm{j}$ \\
\hline $\mathrm{NC}_{\mathrm{i}, \mathrm{j}, \mathrm{m}}$ & Number of subsets of resources for project $\mathrm{i}$, task $\mathrm{j}$, mode $\mathrm{m}$ \\
\hline $\mathrm{NR}_{\mathrm{i}, \mathrm{j}, \mathrm{m}, \mathrm{c}}$ & Number of resources for project $\mathrm{i}$, task $\mathrm{j}$, mode $\mathrm{m}$, subset of resource $\mathrm{c}$ \\
\hline NRP & Number of resource for all projects \\
\hline $\mathrm{NPR}_{\mathrm{r}}$ & Number of priority rules for resource $r$ \\
\hline $\mathrm{NRS}_{\mathrm{s}}$ & Number of resources with skill s \\
\hline$N M Q_{t}$ & Number of current modes in the queue at time $t$ \\
\hline $\mathrm{NIDR}_{\mathrm{z}}$ & Number of time interval partitions during a day for resource $\mathrm{z}$ \\
\hline $\mathrm{NSuc}_{\mathrm{i}, \mathrm{j}}$ & Number of successors for project $\mathrm{i}$, task $\mathrm{j}$ \\
\hline NPred $_{i, j}$ & Number of predecessors for project $\mathrm{i}$, task $\mathrm{j}$ \\
\hline $\mathrm{d}_{\mathrm{i}, \mathrm{j}, \mathrm{m}}$ & Duration time for project $\mathrm{i}$, task $\mathrm{j}$, mode $\mathrm{m}$ \\
\hline$E_{i, j}$ & Eligible date to start for project $\mathrm{i}$, task $\mathrm{j}$ \\
\hline $\mathrm{M}_{\mathrm{i}, \mathrm{j}}$ & Mode chosen for project $\mathrm{i}$, task $\mathrm{j}$ \\
\hline q & Index for current modes in the queue $1<\mathrm{q}<\mathrm{NMQ}_{\mathrm{t}}$ \\
\hline $\mathrm{MQ}_{\mathrm{q}, \mathrm{t}}$ & Mode with index $q$ in the queue at time $t$ \\
\hline $\mathbf{T}$ & interval time, $0 \leq \mathrm{t} \leq \mathrm{T}$ \\
\hline$r n_{i, j, m, c}$ & $\begin{array}{l}\text { Required quantity of renewable resources with skill/capability } \mathrm{c} \text { operating on mode } \mathrm{m} \text { for task } \mathrm{j} \text { of } \\
\text { project } \mathrm{i}\end{array}$ \\
\hline$w_{i, j, m, c}$ & $\begin{array}{l}\text { Required quantity of nonrenewable resource with capability } \mathrm{c} \text { operating on mode } \mathrm{m} \text { for task } \mathrm{j} \text { of } \\
\text { project } \mathrm{i}\end{array}$ \\
\hline$d_{i, j, m}$ & Duration of task $\mathrm{j}$ of project $\mathrm{v}$ operating on mode $\mathrm{m}$ \\
\hline$(i, j, m, c, r)$ & The 5 -tuple $(i, j, m, c, r)$ provides an index re $\in \mathrm{RE}$ \\
\hline$(i, j, m, c, y)$ & The 5 -tuple $(i, j, m, c, y)$ provides an index $\mathrm{nr} \in \mathrm{NR}$ \\
\hline$z_{\left(\operatorname{Re}_{(i, j, m, c, r)}, t\right)}$ & $\begin{array}{l}=1 \text {, if the renewable resource } \mathrm{r} \text { with skill } \mathrm{c} \text { required, operating on mode } \mathrm{m} \text { for task } \mathrm{j} \text { of project } \mathrm{i} \text { is } \\
\text { available at time } \mathrm{t} \text { based on its own calendar } \\
=0 \text {, otherwise }\end{array}$ \\
\hline$z_{\left(N r_{(i, j, m, c, y)}, t\right)}$ & $\begin{array}{l}=1, \text { if the nonrenewable resource } \mathrm{y} \text { with capability } \mathrm{c} \text { required, operating on mode for task } \mathrm{j} \text { of } \\
\text { project } \mathrm{i} \text { is available at time } \mathrm{t} \\
=0 \text {, otherwise }\end{array}$ \\
\hline$d d_{i, i}$ & Assigned due date for task $\mathrm{j}$ of project $\mathrm{i}$ \\
\hline$d d_{i}$ & Assigned due date for project $\mathrm{i}$ \\
\hline$c_{i, i}$ & Tardiness cost task j of project i per time unit \\
\hline$c_{i}$ & Tardiness cost of project i per time unit \\
\hline $\boldsymbol{c s}_{\left(r e_{s r}\right)}$ & Cost associated by using a given skill out of multiples of renewable resource re \\
\hline$c r_{r e}$ & Cost/unit time of renewable resource re in regular time \\
\hline cro $_{\text {re }}$ & Cost/unit time of renewable resource re in over time \\
\hline $\mathrm{crm}_{\text {re }}$ & Cost/unit time of renewable resource re in maintenance \\
\hline$c w_{n r}$ & Cost/unit of nonrenewable resource $\mathrm{nr}$ \\
\hline $\operatorname{car}_{s}$ & Cost for adding a renewable resource with skill s \\
\hline $\operatorname{caw}_{n r}$ & Cost for purchasing one unit of nonrenewable resource $\mathrm{nr}$ \\
\hline$A R_{s, t}$ & Quantity of renewable resources available with skill/capability s at time t \\
\hline$A W_{s, t}$ & Quantity of nonrenewable resource with capability s available (on hand) at time t \\
\hline$(i, j, m, c)$ & The 4-tuple $(i, j, m, c)$ provides an index $\mathrm{s} \in \mathrm{S}$ \\
\hline$A R D_{s, t}$ & Quantity of renewable resources added with skill/capability s at time t \\
\hline$A W D_{n r, t}$ & Total amount of nonrenewable resource $\mathrm{nr}$ delivered at time $\mathrm{t}$ \\
\hline$M R D_{s t}$ & Quantity of renewable resources with skill/capability $s$ in maintenance at time $t$ \\
\hline
\end{tabular}




\begin{tabular}{|c|c|}
\hline$T A W D_{n r}$ & Total amount of nonrenewable resource nr ordered \\
\hline$T A R D_{s}$ & Total quantity of renewable resources added with skill/capability s \\
\hline$T R_{r e}$ & Total amount of time used of renewable resource re in regular time \\
\hline$T W_{n r}$ & Total quantity used of nonrenewable resource nr \\
\hline$T_{R O} O_{r e}$ & Total amount of time used of renewable resource re in over time \\
\hline$T_{R M} M_{r e}$ & Total amount of time in maintenance of renewable resource re \\
\hline$T C_{i, i}$ & Tardiness cost for task $\mathrm{j}$ of project $\mathrm{i}$ \\
\hline$T C_{i}$ & Tardiness cost of project $\mathrm{i}$ \\
\hline $\operatorname{TCS}_{(i, i, m, c, r)}$ & Cost calculated for choosing a given skill out of multiples of renewable resource re \\
\hline TCS & $\begin{array}{l}\text { Total cost associated of choosing a skill out of multiples for all renewable resources, in all modes, } \\
\text { tasks, projects and time. }\end{array}$ \\
\hline TCR & Total cost associated of resources, in all modes, tasks, projects and time. \\
\hline
\end{tabular}

\begin{tabular}{|l|l|}
\hline \multicolumn{1}{|c|}{ Index } & \multicolumn{1}{c|}{ Description } \\
\hline $\mathrm{i}$ & Project index, $1 \leq \mathrm{i} \leq \mathrm{NP}$ \\
\hline $\mathrm{j}$ & Task index, $1 \leq \mathrm{j} \leq \mathrm{NA}_{\mathrm{i}}$ \\
\hline $\mathrm{m}$ & Mode index, $1 \leq \mathrm{m} \leq \mathrm{NM}_{\mathrm{i}, \mathrm{j}}$ \\
\hline $\mathrm{c}$ & Subset index, $1 \leq \mathrm{c} \leq \mathrm{NC}_{\mathrm{i}, \mathrm{j}, \mathrm{m}}$ \\
\hline $\mathrm{r}$ & Resource index, $1 \leq \mathrm{r} \leq \mathrm{NR}_{\mathrm{i}, \mathrm{m}, \mathrm{c}}$ \\
\hline $\mathrm{pr}$ & Rule index, $1 \leq \mathrm{pr} \leq \mathrm{NPR} \mathrm{r}_{\mathrm{r}}$ \\
\hline $\mathrm{s}$ & Skill index, $1 \leq \mathrm{s} \leq \mathrm{NS}$ \\
\hline pre & Predecessor index, $1 \leq$ pre $\leq \mathrm{NPre}_{\mathrm{ij}}$ \\
\hline suc & Successor index, $1 \leq$ suc $\leq$ NSuc $_{\mathrm{ij}}$ \\
\hline $\mathrm{iN}$ & Last task (indexed with $\mathrm{N}$ ) of project $\mathrm{i}$ \\
\hline
\end{tabular}

\begin{tabular}{|c|c|}
\hline Set & Description \\
\hline RE & set of renewable resources, re $\in \mathrm{RE}$ \\
\hline NR & set of nonrenewable resources, $\mathrm{nr} \in \mathrm{NR}$ \\
\hline $\mathrm{RN}$ & set of resources, $r n \in(R E \cup N R)$ \\
\hline I & set of projects, $\mathrm{i} \in \mathrm{I}$ \\
\hline $\mathbf{J}_{\mathbf{i}}$ & set of tasks of project $i, j \in J_{i}$ \\
\hline $\mathbf{P}$ & set of all precedence relationships \\
\hline $\mathbf{M}_{\mathbf{i}, \mathbf{j}}$ & set of modes for task $\mathrm{j}$ of project $\mathrm{i}, \mathrm{m} \in \mathrm{M}_{\mathrm{i}, \mathrm{j}}$ \\
\hline $\mathrm{C}_{\mathrm{i}, \mathrm{j}, \mathrm{m}}$ & set of skills/capabilities operating on mode $m$ of task $\mathrm{j}$ and project $\mathrm{i}, \mathrm{c} \in \mathrm{C}_{\mathrm{i}, \mathrm{j}, \mathrm{m}}$ \\
\hline $\mathbf{R}_{\mathbf{i}, \mathbf{j}, \mathbf{m}, \mathbf{c}}$ & $\begin{array}{l}\text { Set of renewable resources with skill/capability c operating on mode } m \text { of task } j \text {, and project } i \text {, } \\
r \in \mathrm{R}_{\mathrm{i}, \mathrm{j}, \mathrm{m}, \mathrm{c}} \subseteq \mathrm{RE}\end{array}$ \\
\hline $\mathbf{Y}_{\mathrm{i}, \mathrm{j}, \mathrm{m}, \mathbf{c}}$ & $\begin{array}{l}\text { set of nonrenewable resources with capability c operating on mode } \mathrm{m} \text {, for task } \mathrm{j} \text { of project i y } \in \mathrm{Y}_{\mathrm{i}, \mathrm{j}, \mathrm{m}, \mathrm{c}} \\
\mathrm{Y}_{\mathrm{i}, \mathrm{j}, \mathrm{m}, \mathrm{c}} \subseteq \mathrm{NR}\end{array}$ \\
\hline $\mathbf{S}$ & Set of subsets of renewable resources with same skills or capabilities, $s \in S$ \\
\hline $\mathbf{S R e}_{\text {re }}$ & Set of skills for renewable resource re, sr $\in \mathrm{SRE}_{\mathrm{re}}$ \\
\hline
\end{tabular}

\begin{tabular}{|c|c|}
\hline Variable & Description \\
\hline$s t_{i, j}$ & Start time of task $\mathrm{j}$, project $\mathrm{i}$ \\
\hline$f t_{i, j}$ & Finish time of task $\mathrm{j}$, project $\mathrm{i}$ \\
\hline$x_{i, j, m, t}$ & $\begin{array}{l}=1, \text { if task } \mathrm{j} \text { of project } \mathrm{i} \text {, operating on mode } \mathrm{m} \text {, is started at time } \mathrm{t} \\
=0 . \text { otherwise }\end{array}$ \\
\hline $\boldsymbol{h}_{i, j, m}$ & $\begin{array}{l}=1, \text { if task } \mathrm{j} \text { of project } \mathrm{i} \text { is operating on mode } \mathrm{m} \\
=0, \text { otherwise }\end{array}$ \\
\hline $\boldsymbol{u}_{\left(\boldsymbol{R e}_{(i, j, m, c, r)}, t\right)}$ & $\begin{array}{l}=1 \text {, if the renewable resource re with skill } \mathrm{c} \text { required, operating on mode for task } \mathrm{j} \text { of project } \mathrm{i} \text { is } \\
\text { available at time } \mathrm{t} \text { because it is not being used for any other mode among all the projects } \\
=0 \text {, otherwise }\end{array}$ \\
\hline
\end{tabular}




\section{Chapter 1: Introduction and Literature Review}

\subsection{Project Scheduling Methods}

The Project Management Institute defines project as a "temporary endeavor designed to produce a unique product, service, or result" [PMI, 2013]. Temporary implies that projects have a specific start and end dates. Project management is "the process of planning, organizing, motivating, controlling resources, procedures and protocols to achieve specific goals of a project" [PMI, 2013]. Successful conclusion of a project can result in a product, service, or an improved process to deliver a product or a service. Projects exist in every sector of the economy, including manufacturing, healthcare, education, and government. Project Scheduling is a complex task, largely due to multiple tasks, that compete for limited resources. Resources can be skilled labor, capital, equipment, facilities, etc. Significant amount of research has been conducted on allocating limited resources among several competing tasks. Bulk of this research falls under the general area of Operations Research (OR) and Simulation, and the models are either analytical or heuristic.

Project scheduling research has its origins in job shop and flow shop scheduling methods. In order to deal with the complexity of the scheduling problems, authors have suggested a wide variety of techniques over the years. Among these are mathematical programming by Cheng and Hall [Cheng and Hall, 2008], queue rules by Lu et al. [Lu et al., 2010], expert systems by Pesch and Tetzlaff [Pesch and Tetzlaff, 1996], neural networks by Chaudhuri and Kaja [Chaudhuri and Kaja, 2010], multi-agent system by Zhou et al [Zhou et al, 2010], optimized production scheduling by Goldratt [Goldratt, 1988] and supervisory control theory by Pinha et al. [Pinha et al. , 2011].

The scheduling policies and the concept of robustness were addressed by [1- Feng et al., 2012], and [2- Feng et al, 2012]. Briefly, their approach covered buffer capacity, arrival rate based on a Poisson process, operation time and setup time based on the exponential distribution. Their approach provided good scheduling performance for the developed criterion. They utilized seven heuristic dispatching rules 
to determine the overall "best" performance. However, basic production issues, such as, operation precedence and multiple resources required to complete a task were not covered. They demonstrated their approach on a single machine producing multiple products.

Recently, the concept of Transient State (TS) and Steady State (SS), from systems theory point of view, has been applied to production and project planning. Machine failures and repairs cause system perturbations, resulting in transition from SS to TS [Yang and Jingang, 2012]. The TS affects output dynamics, therefore studies have been performed to measure or estimate the time to reach SS from TS. Statistical approaches have been applied to model jobs arrival rates, services rates, the time to machine failure, time to machine repair (gamma distribution) and work in process. Yang [Yang and Jingang, 2012] compared the true dynamic response to the predicted response. Their approach provides average values of outputs with respect to the TS.

Numerous challenges exist when modelling "real world" situations. Several researcher are actively engaged in addressing these challenges [Naber and Kolisch, 2014], [Xu and Feng, 2014]. The research on project scheduling is well ahead of practice. The most common tool that is utilized in industry is Microsoft Project [MS Project, 2015]. This tool has several limitations. It is unable to efficiently handle resource constraints and multiple projects, resulting cost over runs and schedule slippage.

This chapter provides a review of scheduling methods as follows:

1. Program Evaluation and Review Technique - Critical Path Method

2. Resource Constrained Project Scheduling Problem (RCPSP)

3. Multi-mode Resource Constrained Project Scheduling Problem (MRCPSP)

4. Resource Constrained Multi-Project Scheduling Problem (RCMPSP)

5. Resource Constrained Project Scheduling Problem with flexible resource profile (FRCPSP)

6. Multi-mode Resource Constrained Multi-Project Scheduling Problem (MRCMPSP)

7. Project scheduling in the ship repair industry

8. Project scheduling in the construction industry 


\subsubsection{Critical Path Method}

Critical Path Method (CPM) was introduced in the late 1950's [Moder and Phillips, 1964]. This technique represents project as a network using graph theory [Hazewinkel, 2001]. CPM is based on deterministic task time. The Task-on-Node $(\mathrm{ToN})$ diagram is used to describe project tasks. The nodes represent tasks and arrows define the precedence relationship. Figure 1 shows six tasks, with node ST and node EN being dummy start and end tasks, respectively. The number above each node represents task duration. Task 6 in the Figure 1 has 2 incoming arrows while it has only one outgoing arrow. Task 6 has two predecessors (tasks 4 and 5) and only one successor (task EN) [PMI, 2013]. The network is a directed graph and no loop cycles are allowed. CPM assumes no constraints on resources. In spite of the severe assumption this method is widely used in practice, mainly due to its simplicity.

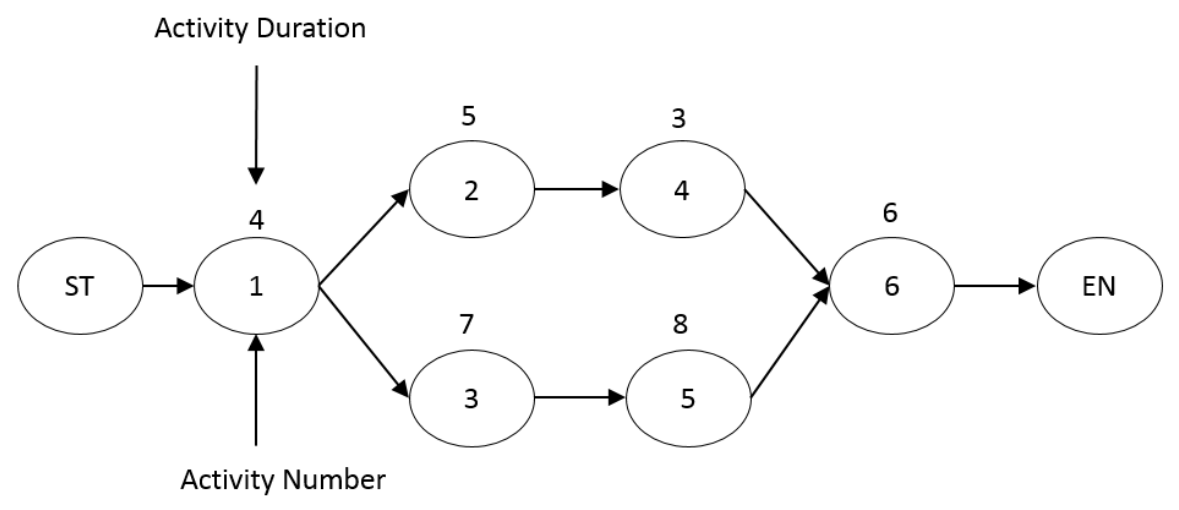

Figure 1: Task on Node Diagram for the Critical Path Method

\subsubsection{Program Evaluation and Review Technique}

Program Evaluation and Review Technique (PERT) was also introduced in the late 1950's [Fazar, 1959]. The Navy's Special Projects Office was charged with the Polaris-Submarine weapon system and the Fleet Ballistic Missile capability. PERT was then developed by a partnership between Booz Allen Hamilton and the U.S. Navy. This technique represents project as a network using graph theory 
[Hazewinkel, 2001]. PERT also utilizes the Task-on-Node (ToN) diagram to describe project tasks. However, PERT allows probabilistic values for task duration time and it assumes that each task duration is a random variable between two extreme values: an optimistic time (a), pessimistic time (b). Also, a realistic time $(\mathrm{m})$ is taken into account. It usually follows a beta probability distribution [Vanhoucke, 2013]. The expected time t of a beta distribution can be approximated by the weighted average shown in equation 1:

$$
t=(a+4 m+b) / 6
$$

Nodes and arrows follow precedence relationship as in CPM. PERT also assume no constraints on resources. In Figure 2, task 3's parameters are $\mathrm{a}=4, \mathrm{~m}=7$ and $\mathrm{b}=9$.

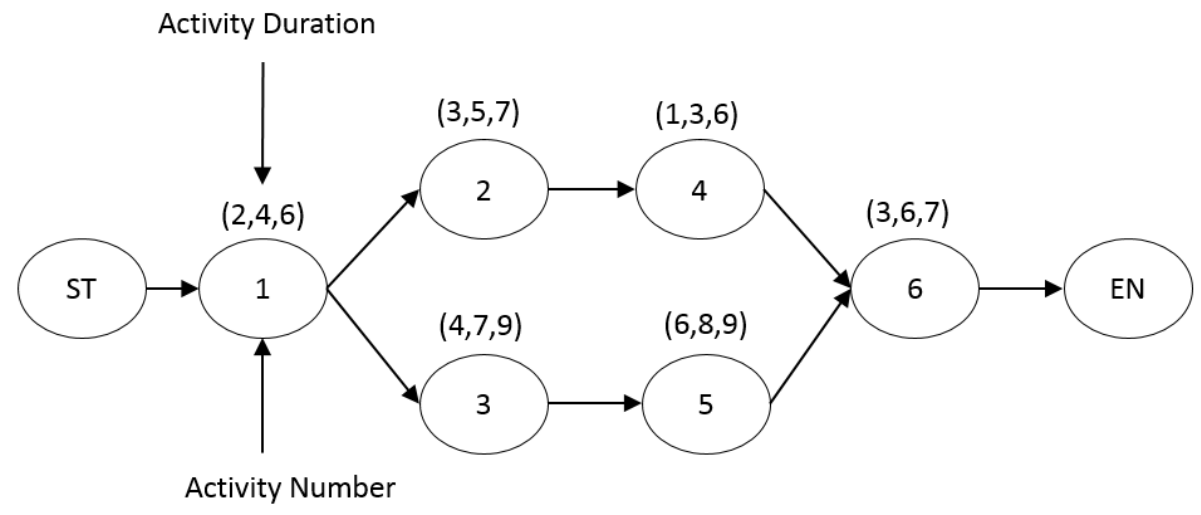

Figure 2: Task on Node Diagram for the PERT

\subsubsection{Resource Constrained Project Scheduling Problem (RCPSP)}

Resource Constrained Project Scheduling Problem (RCPSP) was introduced by Pritsker, Watters, and Wolfe [Pritsker et. al, 1969]. They assumed a single task duration and resource requirement pair. Several variations of the RCPSP method have been proposed. Brucker [Brucker et al, 1999] introduced the notion of limited renewable and non-renewable resources and conflicts between multiple resources. Hartmann and Briskorn [Hartmann and Briskorn, 2010] provided an extensive survey of variants and extensions of the RCPSP. They described two distinct classes of solutions, one for regular measures of 
performance, and the other for non-regular measures of performance. Bianco and Caramia [Bianco, L. and Caramia, 2013] developed an exact formulation for RCPSP.

Vanhoucke [Vanhoucke, 2013] described the above problem mathematically. Its objective function was to minimize the total duration of a project by minimizing the start time of the last task, subjected to precedence relationships and limited resources. Maenhout and Vanhoucke (2015) proposed an exact algorithm to solve the RCPSP integrated with project staffing problem considering different rules depending upon some labour regulations.

Figure 3 shows the Task-on-Node (ToN) network for RCPSP. The numbers and letters above each node represent task duration and resource requirement pair. Table 1 shows hypothetical task duration and resource requirements for three tasks. Each node has only one pair of task duration and resource requirement. This pair is predetermined and fixed, e.g. in Table 1, duration of task 2 is 5 time units and it requires $12 \%$ of resource 1 and $50 \%$ of resource 2. Details of this model are provided in [Vanhoucke, 2013].

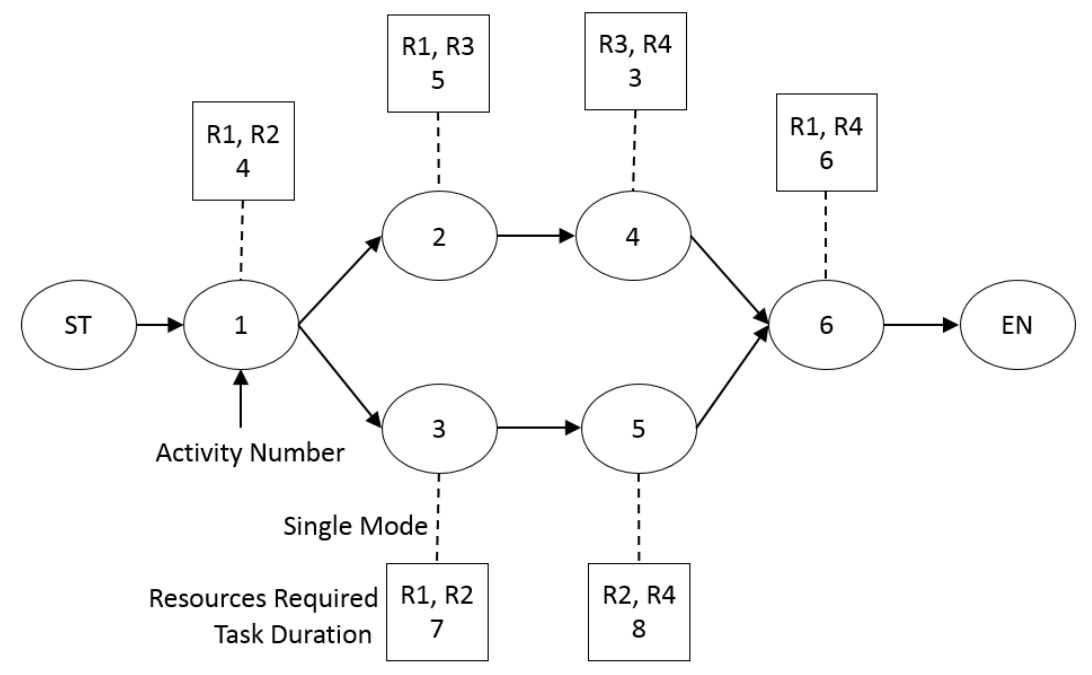

Figure 3: Task on Node Network for RCPSP 
Table 1: Resource requirements

\begin{tabular}{|c|c|c|c|}
\hline Task & Duration & Resource 1 & Resource 2 \\
\hline ST & 0 & 0 & 0 \\
\hline 2 & 5 & 12 & 50 \\
\hline 3 & 7 & 35 & 54 \\
\hline
\end{tabular}

\subsubsection{Multi-Mode Resource Constrained Project Scheduling Problem (MRCPSP)}

Multi-mode Resource Constrained Project Scheduling Problem (MRCPSP) is an extension of the RCPSP method. It was first introduced in 1977 by Elmaghraby [Elmaghraby, 1977]. Difference between RCPSP and MRCPSP is that RCPSP had only one pair of task duration-resource requirements to perform a task, whereas in MRCPSP each task can be performed by selecting one out of several different combinations of task duration-resource requirements. The various combinations are called modes. Naber and Kolisch [Naber and Kolisch, 2014] described mode as "a non-preemptive, constant resource usage of a task over its entire predetermined fixed duration." Figure 4 shows the ToN network for the MRCPSP. E.g. in Figure 4, task 3 has three modes with durations 7, 8, and 10 unit of time, respectively. Table 2 shows task duration-resource requirements and modes for task 3. Each node has task duration-resource requirements pair. The pairs are predetermined and fixed, e.g. task 3 has one mode of seven units of time and it requires resource $\mathrm{R} 1$ and $\mathrm{R} 2$. Table 3 describes additional details.

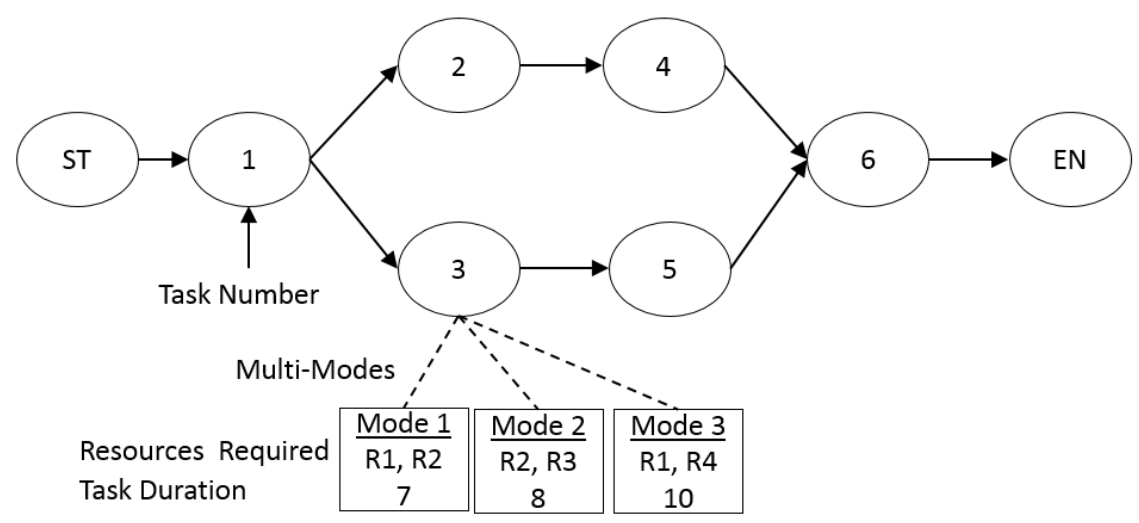

Figure 4: Task on Node for MRCPSP 
Table 2: Modes for task 3

Task

Modes

3

(7, R1, R2), (8, R2, R3), (10, R1, R4)

Table 3: Mode Description

\begin{tabular}{|c|c|c|}
\hline Modes & Resource 1 & Resource 2 \\
\hline$(7, \mathbf{R} 1, \mathbf{R 2})$ & 12 & 50 \\
\hline$(8, \mathrm{R} 2, \mathrm{R3})$ & 35 & 54 \\
\hline$(10$, R1, R4) & 10 & 40 \\
\hline
\end{tabular}

Various authors [Alcaraz and Maroto, 2003], [Bouleimen and Lecocq, 2003], [Hartmann, 2001], [Jarboui et. al, 2008], [Jozefowska et. al, 2001], [Ozdamar, 1999], [Varma et. al, 2007], [Brucker and Knust, 2001], [Calhoun et al, 2002], [De Reyck and Herroelen, 1999], [Drexl et al, 2000], [Heilmann, 2001], [Heilmann, 2003], [Zhu et. al, 2006], [Sabzehparvar and Seyed-Hosseini, 2008], [Peteghem and Vanhoucke, 2010], [Bouleimen and Lecocq, 2003], [Jarboui et al, 2008], [Kolisch and Drexl, 1997] have proposed both heuristic and exact solutions for the above problem.

\subsubsection{Resource Constrained Multi-Project Scheduling Problem (RCMPSP)}

Further extension of RCPSP is Resource Constrained Multi-Project Scheduling Problem (RCMPSP). RCMPSP can handle situations where multiple projects compete for the same resource. In RCMPSP, a task has only one possible combination of duration-resource requirement (one mode), as in RCPSP. However, RCMPSP can handle several projects, and project tasks need to be scheduled simultaneously, under precedence and resources constrains. Browning and Yassine handled RCMPSP by revising priority rules [Browning and Yassine, 2010]. Xue [Xue et al, 2010] applied neural network approach to solve RCMPSP. Zhang and Sun [Zhang and Sun, 2011] used priority-rule based heuristics. Laslo [Laslo and Goldberg, 2008] identified uncertainty in the multi-project environment. Chen and Shahandashti [Chen and Shahandashti, 2009] used the genetic algorithm. Bouleimen and Lecocq [Bouleimen and Lecocq, 2003] utilized the simulated annealing approach. Araúzo [Araúzo et al, 2010] applied Multi-Agent System to the RCMPSP problem. 


\subsubsection{Resource Constrained Project Scheduling Problem with Flexible Resource Profile (FRCPSP)}

The MRCPSP uses a multi-mode approach to perform each task, compared to RCPSP, which has

only one mode. However, both approaches use a fixed pair of task duration-resource requirements. Kolisch [Kolisch et al, 2003] proposed that task duration-resource requirement pair must be determined by the method and should not be fixed. Naber and Kolisch [Naber and Kolisch, 2014] proposed using Mixed Integer Programming (MIP) model to solve what they termed as Resource Constrained Project Scheduling Problem with flexible resource profile (FRCPSP).

Formulation of FRCPSP was also described by Naber and Kolisch [Naber and Kolisch, 2014]. They explained flexible resource profile by an example. That is, if a given task requires 10 person-days, it may be allocated as a constant profile of 2 persons for 5 days (as in the RCPSP), or as a flexible profile of 3 persons for 2 days and 2 persons for 2 days. The authors stated that one can derive FRCPSP as being MRCPSP by building several modes for each task. The issue with this approach is that it only works for discrete resources. For continuous resources, such translation is extremely cumbersome. The authors compared their solution with other exact methods and concluded that efficient solution methods, both exact and heuristic, for real-world applications need to be studied further. The authors also cited an interesting project scheduling problem in the healthcare industry, where FRCPSP can be applied. They regarded fellowship training program as a project, that consists of a number of different surgical type tasks, requiring a certain number of surgical sessions (resources) to be completed over the training period, subject to the pre-requisite requirements (precedence) and limited number of surgeries (resource availability) that the medical fellow can conduct in each period. Other work on FRCPSPs can be found in Fundeling (2006), Fundeling and Trautmann (2010), Ranjbar and Kianfar (2010) and Baumann and Trautmann (2013). 


\subsubsection{Multi-mode Resource Constrained Multi-Project Scheduling Problem (MRCMPSP)}

Many researchers have worked on several variations of the RCPSP, RCMPSP and MRCPSP. However, the case of Multi-mode Resource Constrained Multi-Project Scheduling Problem (MRCMPSP) has not been addressed adequately. The MRCMPSP approach allows several project tasks to be handled simultaneously, under precedence and resources constraints, and each task can have several modes. Thus far, MRCMPSP is the most complex scenario cited in the literature. Only few papers addressed the MRCMPSP. It referred to the studies by Speranza and Vercellis (1993) and by Xu and Feng (2014). Xu and Feng applied a modified particle swarm optimization algorithm as a heuristic method to manage construction of a large-scale hydropower plant. They affirmed that exact methods are not able to solve complex real world problems.

The RCPSP and its more complex extensions are regarded as a standard approach to project scheduling. They belong to the class of strongly NP-hard problems [Kolisch, 1996]. Majority of resource-constrained scheduling methods can broadly be classified as exact or heuristic. Goal of the exact procedures is to find the optimal solution. These methods however, are only suitable for small projects with several assumptions to simplify the problem. Many authors have attempted to incorporate additional capabilities by simulation [Araúzo et al, 2010]. The heuristic methods aim to find a good, and not necessarily the best schedule, using more realistic assumptions [Vanhoucke, 2013].

\subsection{Project Management in Industry}

\subsubsection{Construction Industry}

According to AlSehaimi et al. (2013), the construction industry has lower productivity compared to other industries. Low productivity is largely due to poor resource management, availability of skilled labor, and reliability of the material supply chain. A study by the U.S. National Research Council (NRC 2009) also came to a similar conclusion. They indicated that in order to remain competitive the 
construction industry needs to manage project schedules, labor, material, and energy costs more effectively.

The construction industry, like other industries, has lacked a valid resource-constrained approach to plan their tasks [Lu et. al, 2008]. Popular project scheduling software systems such as Primavera [Primavera, 2015] and Microsoft Project [MS Project, 2015] have been applied in this sector, largely due to absence of a more suitable tool. When resources are scarce and shared along different tasks in complex projects, resource levelling is cumbersome under traditional approaches. Lu [Lu, 2003], Lu et al. [Lu et. al, 2008] utilized on simulation based methods to schedule tasks in the construction industry.

The goal of simulation based modelling is to provide an understanding of problems by building a computational logic of complex real-life problem [Law and Kelton, 2000]. There are two major categories with respect how the system changes overtime, discrete event simulation and continuous simulation [Pritsker and O’Reilly, 1999]. In general, a discrete event simulation is concerned with stimuli named events. The occurrence of an event at discrete points in time modifies the system, thus altering its current state at that time [Pinha et al, 2011]. Majority of simulation application in the construction engineering utilize on the discrete event simulation, a review of discrete event simulation methods in the constructions can be found in [Lau et al, 2014]. Further details of discrete event simulation approach are described in chapter 4.

Other work dealing with real-life projects in construction is described by $\mathrm{Lu}[\mathrm{Lu}, 2003]$. $\mathrm{He}$ developed a simplified discrete-event simulation approach (SDESA) by extracting features from existing simulation methods to plan the dynamic and interactive construction systems. [Lu and Chan Lu, 2004] present an extension of SDESA to deal with resource availability and multiple calendars having only one shift; [Lu et. al, 2008] describe an approach called Simplified Simulation-based Scheduling system (S3) based on SDESA, a new float determination method for CPM and Particle Swarm Optimizer (PSO), and [Lau et al, 2014] presented an approach to discretize continuous resources. Recent work of Siu et al [Siu et al, 2014] propose a bi-level project simulation methodology to first determine the optimal resource 
quantities and the shortest time duration as needed for accomplishing each work package; and second to estimate total project duration and budget through Monte Carlo simulation.

\subsubsection{Ship Repair Industry}

According to [Van Dijk, 2002], the traditional time-driven approaches such as the Critical Path Method (CPM), has several shortfalls for the ship repair industry. Roelof van Dijk [Van Dijk, 2002] worked with The Royal Netherlands Navy Dockyard (RNND) on a ship repair scheduling. RNND is responsible for maintenance, repair, and modifications of the Dutch naval vessels. They claim that production planning and scheduling in shipyards is unreliable. They further state that traditional timedriven approaches such as CPM have several shortcomings. For instance, resource capacity requirements are consequence of plans, but they should have been taken into consideration simultaneously as input with time and costs. Therefore, according to the author, plans performed with CPM are not reliable even for a single project. This situation is worse for the multi-project environment where resources must be shared among multiple projects. The integration of different stakeholders with different levels of responsibility was also mentioned as a critical need.

[Van Dijk, 2002] approach is an extension of the approach proposed by [De Boer,1998], [De Boer et al., 1997]. Wullink's work [Wullink, 2005], [Wullink et al., 2004]. It deals with resource loading under uncertainties. He utilized a scenario based approach and the concept of robustness to deal with demand and capacity uncertainties. He did not consider precedence relationships, release dates, and rush orders. Dlugokecki [Dlugokecki et al., 2010] proposed a project management approach inspired in Ballard [Ballard, 2000]. His work showed improvement in cost savings and higher level of productivity only for building new ships, not for ship repair tasks. Ballard and Choo [Ballard, 2000], [Choo, 2003] presented a resource model to manage construction projects. Their model lacked complexities of the ship repair industry. 
Works of [Mourtzis, 2005], [Chyrssolouris et al., 2004], and [Chyrssolouris, 1999] have integrated different stakeholders in their planning process. They took a systems approach to planning and utilized state of the art information technology tools, such as heuristics and event-driven simulation, to allocate resources. They identified major differences between production planning and scheduling for the ship building industry vs. the ship repair industry. Some of the differences are types of facilities, types of equipment, worker skill levels, work flow patterns, shifting priorities, cost and delivery schedule [Chabane, 2004]. Authors [Charris and Arboleda, 2013], [Mello and Strandhage, 2011] worked on supply chain management for shipyards. [Pinha et al, 2011] presented an application of the supervisory control theory to schedule ship repair tasks. Preliminary work was described in [Pinha and Ahluwalia, 2014] and [Pinha and Ahluwalia, 2013]. The authors designed a database schema for the ship repair industry and proposed Discrete-Event-Simulation model. Zhou [Zhou et al., 2013] proposed solutions for repairing war ships; however, their work lacked several real constraints. Papakosta's approach [Papakostas et al., 2010] was based on [Chryssolouris and Dicke, 1992], [Chryssolouris et al., 1992, 1991], [Chryssolouri, 2005] for scheduling maintenance of airplanes whereas [Framinan and Ruiz, 2012, 2010], [Moghaddam and Usher, 2011], and [Yamashita et al, 2007] proposed alternate approaches.

Numerous gaps exist with respect to modeling of ship repair facilities. Currently, majority of production planning and scheduling task are considered static in nature and utilize Microsoft Excel or Microsoft Project software to scheduling, often resulting in cost over runs, schedule slippage, and low throughput. Traditional project management approach uses a pre-determined approach to resource allocate. The flexibility in resource allocation based on worker skills can reduce costs and increase throughput. This is an urgent need in the ship repair industry [DoN, 2013], [NSRP, 2013], [Leadership, 2013], [MARAD, 2013]. Also, there is a need for an integrated tool for resource allocation dealing with multi-modes, multi-projects, and resource constraints.

Application of project scheduling techniques in this industry has been very limited. US and European governments have identified project scheduling in shipyards as a major area of concern [NSRP, 
2013], [Leadership, 2013], [MARAD, 2013]. Scheduling in the ship repair industry is further complicated due to unexpected demand, changing priorities, lack of skilled workers, high capital investment in specialized equipment, and environmental issues [Dlugokecki et. al., 2010], [Wullink, 2005], [Wullink et. al., 2004].

\subsubsection{Limitations of the Commonly Used Tools}

Table 4 summarizes pros and cons of some the scheduling approaches described in literature.

\begin{tabular}{|c|c|c|c|}
\hline \multicolumn{4}{|c|}{ Table 4: Pros and Cos } \\
\hline Class & Pros & Cos & Authors \\
\hline $\begin{array}{l}\text { Mathematical } \\
\text { Formulations }\end{array}$ & 1-Exact solutions & $\begin{array}{l}\text { 1. Applied to small problems } \\
\text { constraints } \\
\text { 2. Many assumptions }\end{array}$ & $\begin{array}{l}\text { [Vanhoucke, 2013] } \\
\text { [Naber and Kolisch, } \\
\text { 2014] }\end{array}$ \\
\hline $\begin{array}{l}\text { Optimization } \\
\text { with Heuristics }\end{array}$ & $\begin{array}{l}\text { 1-Fuzzy } \\
\text { operation times }\end{array}$ & $\begin{array}{l}\text { 1. Lack of daily calendar } \\
\text { 2. Only Finish-to-Start, no lag- } \\
\text { times } \\
\text { 3. Limited few tasks and resources } \\
\text { 4. Limited to single skill or } \\
\text { operation }\end{array}$ & [Xu and Feng, 2014] \\
\hline $\begin{array}{l}\text { Discrete Event } \\
\text { Simulation }\end{array}$ & $\begin{array}{ll}\text { 1. } & \begin{array}{l}\text { Determine } \\
\text { resource } \\
\text { requirement }\end{array} \\
\text { 2. } & \begin{array}{l}\text { Estimate total } \\
\text { project } \\
\text { duration }\end{array} \\
\text { 3. } & \text { Probability of } \\
\text { scenarios to } \\
\text { estimate } \\
\text { durations }\end{array}$ & $\begin{array}{l}\text { 1. No modes nor multi-projects } \\
\text { 2. No dynamic queues (first in - } \\
\text { first out) } \\
\text { 3. No multiple priority rules } \\
\text { 4. No multiple-skills for resources }\end{array}$ & $\begin{array}{l}\text { [Lu, 2003] } \\
\text { [Lu and Chan Lu, 2004] } \\
\text { [Lu, Lam, and Dai, } \\
\text { 2008] } \\
\text { [Lau et al, 2014] } \\
\text { [Siu et al, 2014] } \\
\text { [Mourtzis, 2005] } \\
\text { [Chyrssolouris et al., } \\
\text { 2004] } \\
\text { [Chyrssolouris, 1999] }\end{array}$ \\
\hline $\begin{array}{l}\text { Microsoft Project } \\
\text { and Primavera }\end{array}$ & $\begin{array}{l}\text { 4. Friendly user } \\
\text { interface } \\
\text { 5. Levelling } \\
\text { Resources } \\
\text { based on } \\
\text { rules } \\
\text { 6. Central } \\
\text { database for } \\
\text { managing } \\
\text { multiple } \\
\text { projects }\end{array}$ & $\begin{array}{l}\text { 5. No modes } \\
\text { 6. No multiple priority rules for } \\
\text { resources } \\
\text { 7. No multiple-skills for resources } \\
\text { 8. Faulty interpretation in handling } \\
\text { multiple calendars for resources } \\
\text { 9. Limited quantity of time } \\
\text { intervals per day (5) } \\
\text { 10. Does not allow task duration } \\
\text { with less than one minute. } \\
\text { 11. Only one rule applied to all } \\
\text { resources. }\end{array}$ & $\begin{array}{l}\text { [MS Project, 2015] } \\
{[\text { Primavera, 2015] }}\end{array}$ \\
\hline
\end{tabular}


A recent work of Coughlan et al. (2015) utilized the Branch-Price-and-Cut algorithm for Multimode resource levelling to solve instance of problems with 50 jobs. The authors stated that even today instances with only 30 jobs are hard to solve to optimality. Also, a survey performed by Rehm and Thiede (2012) at Technische Universität Dresden about project scheduling methods presents interesting results. Rehm and Thiede state that since 1981, several solutions methods have been proposed. Since 1993, an average of 3 new solutions a year have been published. A relevant finding of their survey is that majority of solutions methods proposed are able to handle up to 51 tasks, and they utilize heuristics and use genetic algorithm. Regards to their objectives, most of the solutions are time-oriented and do not consider several resource constraints. No solution was found that covered all real-life production concerns. Rehm and Thiede state that due to innumerous existing gaps, further research is necessary. The authors conclude that due to the importance of this field, study will persist and new methods are likely to continue in the coming years.

Microsoft Project [MS Project, 2015] is certainly one of the most common software tool used in the industry. It is relative easy to use. However, resource allocation for competing tasks (levelling resources) is limited with only three general rules. Primavera [Primavera, 2015] is another software tool widely used in the industry. It can handle large data sets. It utilizes Oracle Database [Oracle, 2015], resource allocation for competing tasks is limited and cumbersome in Primavera.

Within the context of project scheduling, modelling of real world situations is a challenging task. Araúzo et al (2010) stated that classical methods, based on mathematical programming, could help decision making when problem complexity is low and the system is somewhat static. On the other hand, these characteristics are seldom true in real world projects. Microsoft and Primavera Project Planner (P3 and P6 versions), which are the most common tools used for project management, present shortcomings on relevant issues in some contexts. For instance, they do not guarantee accuracy in results when tasks require multiple resources that have different calendars associated. Faulty interpretations in calculating finish time for tasks exist. They do not support multiple task modes (i.e., only one set of resources for 
each task). They lack an efficient and automatic resource allocation procedure; they simply adopt fixed standard rules for levelling all resources in a context where rules may differ among the organization departments. These shortcomings reinforce the need for techniques to properly assist project management.

ProChain [ProChain, 2015] and ProTrack [ProTrack, 2015] are also used tools. ProChain has been applied more often in US projects whereas ProTrack is used in European countries. ProTrack simulates time and cost trade-offs, however neither multi-mode nor multi-project is offered. ProChain has been developed by a consulting company in US and technical support and/or customizations to attend customer needs are offered. ProChain allows lag times, but no resource allocation is done for competing tasks.

In a recent action research, Abrantes and Figueiredo (2015) worked closely with industrial partners and brought strong empirical evidence that resource management is indeed a concern in organizations driven by projects. They presented the challenges in resource management for real-life multiple-projects contexts, characterized by high dynamism and dependency complexity among projects. Resource management methods have received large attention in the academic literature, however resource management approaches to support organizations are still not established [Abrantes and Figueiredo , 2015].

Maenhout and Vanhoucke (2015) stated as future research in their work: "We aim to develop solution techniques that are able to tackle real-life problems. This means that on the one hand, we should increase the problem size (in terms of number of activities) scheduling one or multiple projects. On the other hand, we should expand the problem definition and incorporate additional personnel and task characteristics such as personnel skills, multiple modes of operation for a task, task pre-emption, etc. Exact optimization techniques will not be able to cope with the further extension of the problem definition and size. For this reason, we will focus on (hybrid) heuristic optimization techniques that combine the advantages of both mathematical programming techniques and meta-heuristics optimization”. 


\section{Chapter 2: Research Objectives}

\subsection{Introduction}

All sectors of the economy require project management. In spite of differences in industries such as constructions, ship repair, manufacturing, healthcare, and education, there are many similarities with respect to project management, e.g. how to manage and how to allocate scarce resources is an issue found in all sector. Maintenance/Repair of ships, civil constructions, production of specialized equipment for industries such as coal, petroleum, power plants, and wind plants are examples of production environment driven by projects. Typically, project delays result in huge financial losses for the customer.

Project lead times depend not only on its own task times, but also due to waiting time in queues due to lack of resource. Thus, managers need to account for the current status of projects, and the current availability of resources to come up with project duration. In multi-project environment, this assessment is difficult because the number of tasks is very high and resource availability is unstable. Current resource availability requires a detailed assessment which is difficult to perform with traditional project management approaches. Tables 5 and 6 lists typical project planning attributes [Costa and Jardim, 2010].

Table 5: Project planning attributes

\begin{tabular}{|l|l|}
\hline \multicolumn{1}{|c|}{ Attributes } & \multicolumn{1}{c|}{ Values } \\
\hline Demand Pattern & Customer orders \\
\hline Production volume & Low. Usually as a unit \\
\hline Production frequency & None or low repetitive \\
\hline Services & Opened/including innumerous services \\
\hline Production mix & High unstable \\
\hline Lead times & High \\
\hline Resources layout & Functional, Fixed-Position \\
\hline
\end{tabular}




\section{Table 6: Typical issues for planning and controlling}

\begin{tabular}{|l|l|}
\hline \multicolumn{1}{|c|}{ Typical } & \multicolumn{1}{c|}{ Obstacles } \\
\hline Scheduling & Search for an appropriate solution in a virtually infinite universe of possibilities \\
\hline Short-term & Complex assessment of cost-benefit being made under intense pressure on a daily basis \\
\hline Deadline & Lack of previous data and lead times depends on the task mix \\
\hline Budget & Lack of previous data and profitability dependent on capacity adjustments \\
\hline Re-planning & Instability are facts intrinsic in the project environment \\
\hline Production & Need for controlling specific task dealing with large volume of data \\
\hline
\end{tabular}

An approach which supports multiple combinatorial modes and multiple projects is needed. In several projects, tasks can be performed by selecting one out of several different set of resources. Usually, a project manager is in charge of one project, when multi-projects must be run simultaneously, different plans (projects) exist. These sub-plans frequently results in sub-optimization of overall plan, resulting in lower productivity. Required resources such as workers, tools, materials are not adequately shared among projects, resulting in conflicts along managers and schedule slippage.

There is a need to support large scale, real-life projects, where people have multiple skills and equipment can perform a variety of tasks with varying efficiency levels. Methodologies suitable for dealing with multiple projects on a daily basis (differing in terms of size, resource constrained, and in considering real status of each task) remains the main challenge for scheduling. Also, one of the gaps identified from the literature is how to model production capacity of projects. If the capacity is not well described, the results might not represent accurately the production reality.

Assuming tasks require multiple skills, the model must be able to find from the pool of resource those which are able to perform tasks having the skills required. By not allowing multi-skills for resources, increases cost and reduces productivity. In addition, if no relationship exists between a resource and its skills, it is not possible to have resource allocation based on skills. This gap exists mainly due to the traditional project management approach to individually deal with time, cost and capacity. It results in complicating the planning process and ultimately it has a negative impact on productivity. 
Why project scheduling is hard? Wall (1996) identified four different project scheduling issues that make it difficult.

1) The Size of the Problem: The size of the problem scheduling can be approximated by what-wherewhen matrix. Tasks (What) require resources (Where) for certain period of times (When).

2) Modelling issues: An optimum solution under incorrect assumptions is not very useful. A feasible solution under realistic constraints is what practitioners seek.

3) The dynamic nature of problems: Real world situations are unpredictable (machine breakdowns, human absence, inclement weather, etc.). In such cases the pre-planned project schedule are of little value.

4) Infeasible Solutions: If required renewable resources are available during the entire project duration, some delays still occur, but feasible solutions are certainly found. However, if nonrenewable resources exist, and they are scarce, feasible solution cannot be guaranteed.

\subsection{Research objectives}

The overall research objective of this study was to reduce total project duration and cost by providing various alternatives to resource allocation. This approach is based on the notion that project constraints are not constant. The project manager is provided several feasible solutions, which can include the “optimal" solution. Given the dynamic nature of projects, project managers are better equipped to choose one of the several solutions to attend their current needs. The details of specific research objectives are provided below:

Objective 1: Extension of the RCPSP model: The existing RCPSP approach to resource allocation in project scheduling was extended to deal with practical aspects of project scheduling. The concept of mode was extended to include multiple skills of varying levels of competence. A mode is no longer only a set of required resources capable to execute a task. Mode is also a set of combinatorial subsets of required 
resources capable to execute a task. The revised extension model is called Combinatorial Multi-Mode Resource Constrained Multi-Project Scheduling Problem (CMRCMPSP).

Objective 2: Focus on short-term project management: Most existing project management systems take a long term view of the problem. They assume a static system. This work focused on the short-term scheduling problem. Short-term is significantly more complex than long term scheduling, primarily due to need to respond to perturbation in near real time. It allows project manager decide on timely manner which solution among several is the good one to attend his/her current needs.

\section{Objective 3: Provide a capability to support multi-modes, multiple skills, and multiple calendars:}

Short term needs such as multiple calendars, and constraints on resources are addressed. Project scheduling in industry is mainly carried out by applying traditional approaches, using tools such as Microsoft Project or Primavera. In construction industry, project managers commonly add resources constraints such as resource limitations and resource calendars. When projects are subjected to this kind of resource constraints, the correct project duration value cannot be determined by using mainstream software such as Primavera and MS Project.

The above objectives will assist project managers in dealing with unexpected events, queue lengths, priority rules, and user inputs, in near real time. Project scheduling strategies on a daily basis are volatile and unpredictable. They depend upon changing customer requirements and resource availability. Unexpected events always exist, and therefore, handling these events is critical to effectively running dayto-day operations. Decisions made yesterday may not be feasible today. The difficulty of exact solutions is in part due in articulating objective function or functions that represent the overall objectives and are dynamic in nature. Classical methods, based on mathematical programming, can help to make decisions when problem complexity is low and the system is static. These characteristics seldom represent project reality. 


\section{Chapter 3: Method}

This chapter describes the solution method in four parts: (1) the Combinatorial Multi-Mode Resource Constrained Project Scheduling Problem (CMRCMPSP) as an extension of the RCPSP, (2) the problem model in the form of assumptions about tasks and resources with their associated constraints, (3) the simulation method, and (4) a description of the overall system architecture.

\subsection{The Combinatorial Multi-Mode Resource Constrained Multi-Project Scheduling}

\section{Problem}

The Combinatorial Multi-mode Resource Constrained Multi-project Scheduling Problem (CMRCMPSP) is general and its covers the RCPSP, and its main extensions such as MRCPSP, RCMPSP, and MRCMPS. The choice of representation of a problem impacts its complexity and the search space (schedule options). A very specific representation significantly reduces the size of the search (options), and works on only a single problem instance. Nonetheless, a general representation, allows for more types of problems may be solved at the expense of searching a larger space.

The MRCPSP and MRCMPS can be performed by selecting one out of several different combinations of resource requirements (modes). The proposed method allows for unlimited modes. It first attempts to assign the required resources in the first mode (mode 1) for tasks, in case a required resource of mode 1 is not available, then it attempts to assign the required resources of mode 2 , this logic is applied to all modes. Ultimately, if no resource requirements are met for at least one mode out of the possible set of modes of a given task, then it must wait until resource requirements are met for at least one mode. The sequential mode order allows project manager to assign ranks for different modes. The multiple modes flexibility covers the concept of resource-driven task duration defined in Wongwai and Malaikrisanachalee (2011), where tasks can start with partial resource requirements fulfilment. In the proposed method, a given task can have several modes, one of them may represent the least resource requirements to start a task. 
Depending upon the application, the mode order may represent quality, speed, cost, etc. Project manager defines number of modes for each task according to current needs. Mode durations are independent of resource requirements by other modes. For instance, if mode 1 requires more resources in comparison of other modes, does not imply that duration of mode 1 will be shorter, it may be longer or shorter depending upon the process. Less equipment or fewer labours may work more efficiently than several other equipment or labours. Multiple modes provide additional flexibility in developing project schedules.

Many researchers have worked on several variations of the RCPSP such as RCMPSP and MRCPSP. The case of Multi-mode Resource Constrained Multi-Project Scheduling Problem (MRCMPSP) has not been addressed adequately due to its size and complexity. This research extends the MRCMPSP by proposing a new and general extension of all the aforementioned extensions of the RCPSP. The Combinatorial Multi-Mode Resource Constrained Multi Project Scheduling Problem (CMRCMPSP) is created. The proposed CMRCMPSP is capable to solve RCPSP, RCMPSP, MRCPSP, and MRCMPSP. Recall that MRCMPSP is the general extension of RCPSP found in literature and it allows several project tasks to be handled simultaneously, under precedence and resources constraints, and each task can have several modes. The figure 5 shows only one project with 6 tasks with start node (ST) and end node (EN) for illustrative purposes. Each task can have multiple modes, e.g., task 3 has 3 modes. Task 3, mode 1 requires resources R1 and R2 and the task 3's duration operating under this mode is 7 units of time. Task 3, mode 2 requires resources R2 and R3 and the task 3's duration operating under this mode is 8 units of time. Task 3, mode 3 requires resources R1 and R4 and the task 3's duration operating under this mode is 10 units of time. 


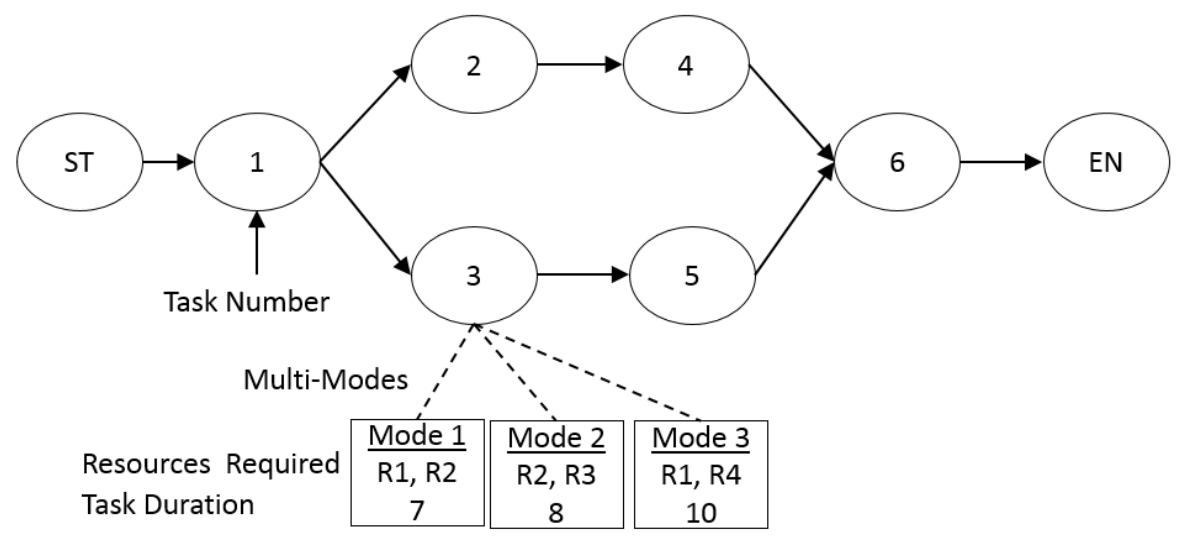

Figure 5: Task on Node of MRCMPSP model

The Combinatorial Multi-Mode Resource Constrained Multi Project Scheduling Problem (CMRCMPSP) allows several project tasks to be handled simultaneously, under precedence and resources constraints, and each task can have several modes. Naber and Kolisch [Naber and Kolisch, 2014] describe mode as a set of resources for executing a task over its entire predetermined fixed duration. In MRCMPSP and CMRCMPSP each task can be performed by selecting one out of several different modes. However, CMRCMPSP differs from MRCMPSP, because a mode in CMRCMPSP is no longer a set of resources only, but it is a set of combinatorial subsets of unknown required resources capable of executing a given task. In order to illustrate how CMRCMPSP differs from MRCMPSP a small example is shown in the Figure 6. It shows one project with 6 tasks along a start node (ST) and an end node (EN). Each task can have multiple modes, e.g., task 3 has 2 modes, mode 1 requires 2 welders, 3 cutters, R12, and nonrenewable resource R15. Task 3's duration under mode 1 is 10 units of time. Task 3, mode 2, requires 3 welders, 1 cutter, R13 and R15 and the task 3's duration operating under mode 2 is 12 units of time. 


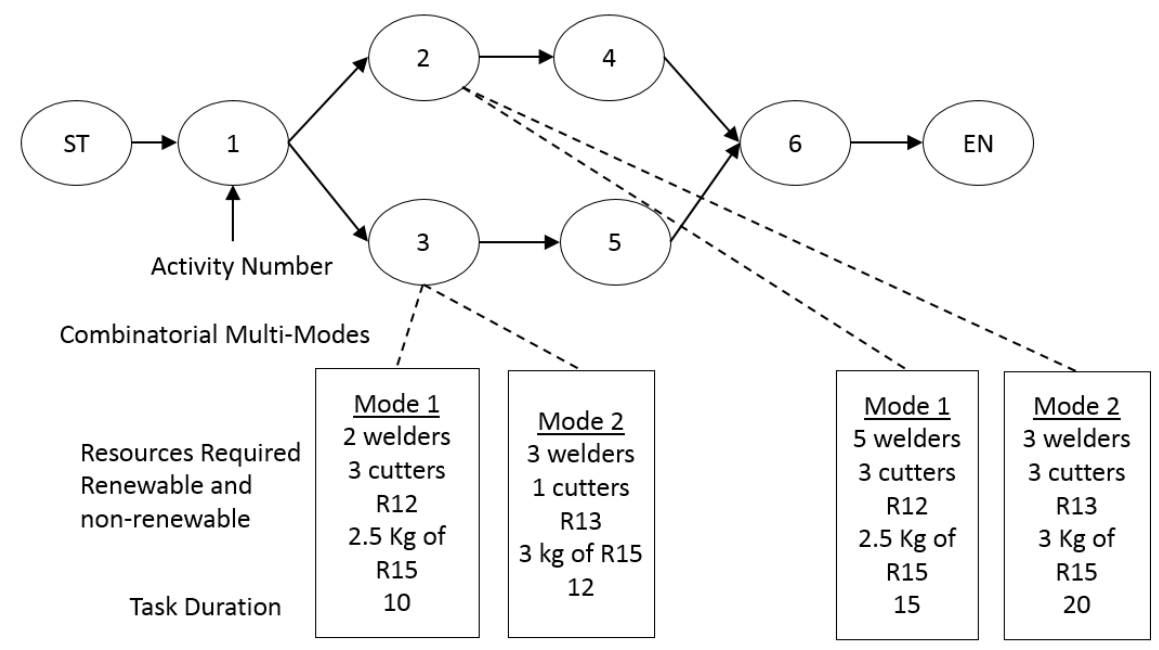

Figure 6: Task on Node of CMRCMPSP model

Table 7 describes the resources, their associated skills levels and their types (renewable or nonrenewable). Notice that some resources can have more than one skill associated with them. The set of combinatorial subsets of unknown required resources can be formed with single-skilled resources in CMRCMPSP. The multi-skilled resources are used in this example to adhere to project environment reality. Very few approaches are capable of handling multi-skilled workers.

The various values in the tables show skill levels (1-Excelent, 2- Good, 3-Reasonable, 4-Poor). For instance, R1 has 3 skills, welding at skill level 1, cutting at skill level 2 and painting at skill level 4. The same logic is applied to all other resources. If no skill value is assigned to a resource, it is assumed to have no skills. Last row of Table 7 shows that there exist 8 welders, 6 cutters and 6 painters in the resource pool. 
Table 7: Resource and Skill

\begin{tabular}{|c|c|c|c|c|}
\hline Resources & Welding & Cutting & Painting & Type \\
\hline R1 (worker 1) & 1 & 2 & 4 & Renewable \\
\hline R2 (worker 2) & 1 & & & Renewable \\
\hline R3 (worker 3) & 1 & & & Renewable \\
\hline R4 (worker 4) & & & 1 & Renewable \\
\hline R5 (worker 5) & & 1 & 3 & Renewable \\
\hline R6 (worker 6) & & 1 & & Renewable \\
\hline R7 (worker 7) & 1 & 2 & 3 & Renewable \\
\hline R8 (worker 8) & 1 & 2 & 3 & Renewable \\
\hline R9 (worker 9) & 1 & & & Renewable \\
\hline R10 (worker 10) & 1 & & 2 & Renewable \\
\hline R11 (worker 11) & 1 & 2 & & Renewable \\
\hline R12 (Machine 1) & & & & Renewable \\
\hline R13 (Machine 2) & & & & Renewable \\
\hline R14 (Machine 3) & & & & Non-renewable \\
\hline R15 (Electrode) & & & & \\
\hline Quantity & 8 & 6 & 6 & \\
\hline
\end{tabular}

CMRCMPSP employs the term combinatorial due to the fact it allows for a finite combinatorial number of options to execute tasks. This differs widely from a standard multi-mode approach because number of modes does not define number of different options to perform a task. In the previous extension of multi-mode RCPSP, a given task with two modes has only two options for being executed. However, in the proposed approach a task with two modes, as it will be shown, can provide more than two options. In Figure 6, Task 3, Mode 1 requires 2 welders out of 8, 3 cutters out of 6, and resources R12, R15. Task 3 , Mode 2 requires 3 welders out of 8,1 cutter out of 6 , and resources R13, R15. In this research, the combinatorial mode is defined as a set of combinatorial subsets of required resources. Task 3 , Mode 1 , the 2 welders required is the first subset, the 3 cutters is the second subset, and so on so forth. For each required subset, a search among a combinatorial number of resources is described by equation 1 :

$$
\left(\begin{array}{l}
n \\
p
\end{array}\right)=\frac{n !}{(n-p) ! p !}
$$


The number of combinations $\mathrm{p}$ of $\mathrm{n}$ elements where $\mathrm{p}$ is the required quantity of resources with a given skill and $\mathrm{n}$ is the available quantity of resources in the resource pool capable of meeting the required skill. In the above example, Task 3, Mode 1 requires 2 welders out of 8,3 cutters out of 6 , R12, and R15. Task 3, Mode 2 requires 3 welders out of 8,1 cutter out of $6, \mathrm{R} 13$, and R15. There are a total of 896 different ways to perform Task 3, not just 2. Equation (2) shows the number of alternative combinations for welders in mode 1 :

$$
\left(\begin{array}{l}
8 \\
2
\end{array}\right)=\frac{8 !}{(8-2) ! 2 !}=28
$$

Equation (3) shows the number of different combinations for cutters in mode 1.

$$
\left(\begin{array}{l}
6 \\
3
\end{array}\right)=\frac{6 !}{(6-3) ! 3 !}=20
$$

$\left(\begin{array}{l}8 \\ 2\end{array}\right)\left(\begin{array}{l}6 \\ 3\end{array}\right)=560$ different sets of resources to perform Task 3 by selection Mode 1

Equation (4) shows the number of alternative combinations for welders in mode 2.

$$
\left(\begin{array}{l}
8 \\
3
\end{array}\right)=\frac{8 !}{(8-3) ! 3 !}=56
$$

Equation (5) shows the number of different combinations for cutters in mode 2.

$$
\left(\begin{array}{l}
6 \\
1
\end{array}\right)=\frac{6 !}{(6-1) ! 1 !}=6
$$

$\left(\begin{array}{l}8 \\ 3\end{array}\right)\left(\begin{array}{l}6 \\ 1\end{array}\right)=336$ different set of resources to perform Task 3 by selection Mode 2

Therefore, there are $(560+336) 896$ alternate ways to perform task 3. A general equation for calculating the number of options to perform a task in the CMRCMPSP is given by equation 6 .

$$
\sum_{m \in M_{i, j}}\left(\prod_{c \in C_{i, j, m}} \frac{n !}{(n-p) ! p !}\right)
$$


For the above example, for Mode 1, the number of combination of welders and cutters is given by $\left(\begin{array}{l}8 \\ 2\end{array}\right)\left(\begin{array}{l}6 \\ 3\end{array}\right)=560$, whereas for Mode 2 the number of combination of welders and cutters is given by $\left(\begin{array}{l}8 \\ 3\end{array}\right)\left(\begin{array}{l}6 \\ 1\end{array}\right)=336$

$\mathrm{m} \in \mathrm{M}_{1,3}=\{$ Mode 1 , Mode 2 $\}$

$c \in C_{1,3,1}=\{2$ welders out of 8,3 cutters out of $6, R 12, R 15\}$

$c \in \mathrm{C}_{1,3,2}=\{3$ welders out of 8,1 cutter out of $6, \mathrm{R} 13, \mathrm{R} 15\}$

$$
\begin{aligned}
& \operatorname{rn}_{(\mathrm{v}, \mathrm{j}, \mathrm{m}, \mathrm{c})}=\operatorname{rn}_{(1,3,1,1)}=2 ; \mathrm{rn}_{(1,3,1,2)}=3 \\
& \sum_{m \in M_{i, j}}\left(\prod_{c \in C_{i, j, m}} \frac{n !}{(n-p) ! p !}\right)=\frac{8 !}{(8-2) ! 2 !} * \frac{6 !}{(6-3) ! 3 !} * 1 * 1+\frac{8 !}{(8-3) ! 3 !} * \frac{6 !}{(6-1) ! 1 !} * 1 * 1 \\
&=896
\end{aligned}
$$

Current literature does not address the above combinatorial problem.

\subsection{The problem model}

Figure 7 provides an overview of the problem model. It shows three simultaneous projects (separated by a dotted line). Each task, represented by a node, can have multiple modes, e.g., Task 3 of Project 1 has two modes. Each mode can require subsets of resources as described in the previous section. Resource can be renewable or non-renewable. In Figure 7, double circle for Tasks 1 of Project 1 indicates that some work has already been performed on this task and the model takes that into account. Tables 8 and 9 describe the proposed solution method in terms of task and resource. 


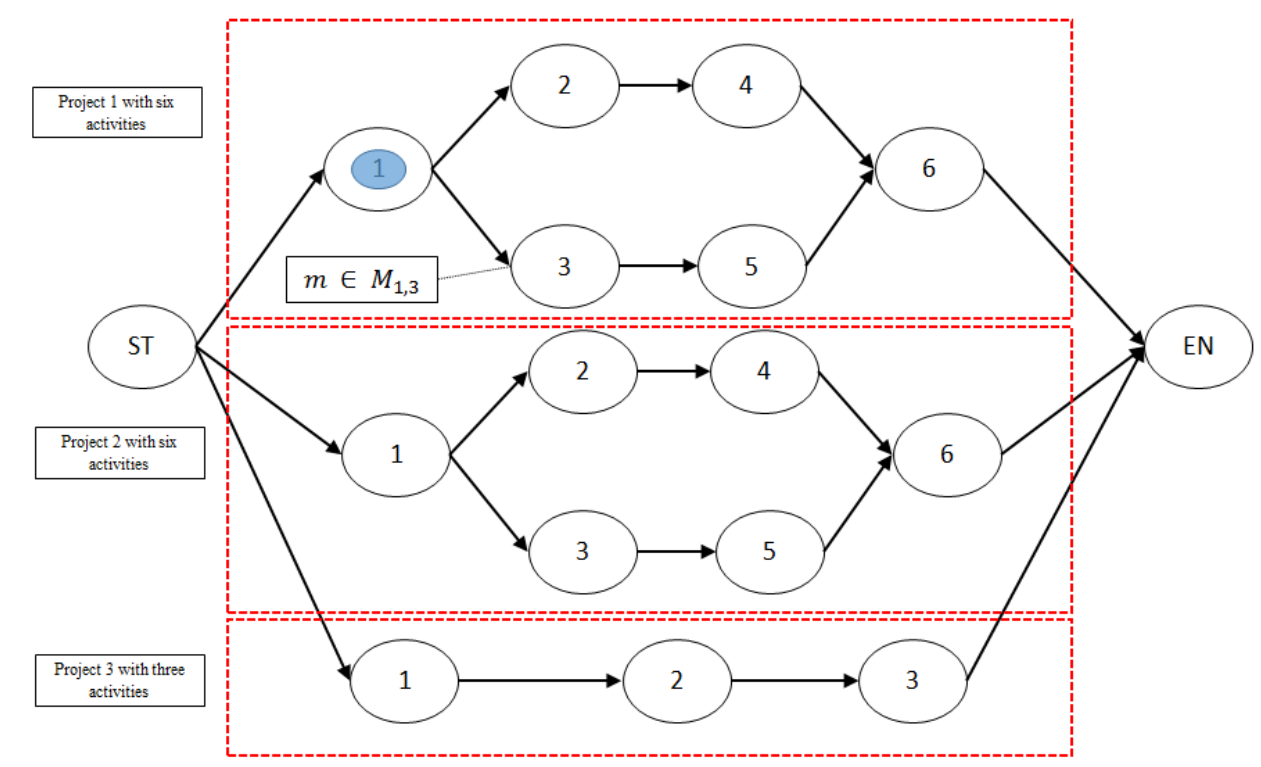

Figure 7: Task on node representation for multiple projects

\begin{tabular}{|l|l|}
\hline \multicolumn{1}{|c|}{ Table 8: Task Modelling } \\
\hline Modelling & \multicolumn{1}{c|}{ Description } \\
\hline Multi-projects & Tasks can belong to several different projects \\
\hline Combinatorial & Tasks can have multiple modes. Each mode has its own subsets of resources. \\
\hline Precedence & Tasks follow a precedence relationship. \\
\hline $\begin{array}{l}\text { Temporal } \\
\text { Interruptions. }\end{array}$ & $\begin{array}{l}\text { Tasks may have temporal constraints such that they can only be done at a certain } \\
\text { time. }\end{array}$ \\
\hline
\end{tabular}

\begin{tabular}{|l|l|}
\hline \multicolumn{1}{|c|}{ Modelling } & \multicolumn{1}{c|}{ Table 9: Resource Modelling } \\
\hline Multi-skilled & Rescription \\
\hline Multiple calendars & Each resource can have its own calendar. \\
\hline $\begin{array}{l}\text { Renewable, non- } \\
\text { renewable } \\
\text { resources, doubly } \\
\text { constrained } \\
\text { resources }\end{array}$ & $\begin{array}{l}\text { There are three types of resources. Renewable resource are not consumed, e.g. } \\
\text { facilities, equipment, workers, etc. Nonrenewable resource is a resource that is } \\
\text { consumed when a task is carried out, e.g. raw material, power, fuel, etc. A plan of } \\
\text { nonrenewable resources is considered. Delivery dates of non-renewable resources } \\
\text { are considered in this model (doubly constrained resource) }\end{array}$ \\
\hline $\begin{array}{l}\text { Multiple priority } \\
\text { rules }\end{array}$ & $\begin{array}{l}\text { Each resource can have its own list of priority rules, e.g., rule for resource 1 } \\
\text { (welding resource for example) do not apply to resource 2 (painting worker) and } \\
\text { vice-versa. }\end{array}$ \\
\hline $\begin{array}{l}\text { Maintenance and } \\
\text { Over times are } \\
\text { allowed }\end{array}$ & $\begin{array}{l}\text { Each resource can have maintenance and idle times. Human resources can have } \\
\text { overtime. }\end{array}$ \\
\hline
\end{tabular}




\subsection{Mathematical formulation of the CMRCMPSP model}

The focus of most current approaches is to formulate the problem as an optimization problem with a goal of finding the optimal solution. Several assumptions are made in order to reduce mathematical complexity. Various models use heuristic or a genetic algorithm to make the problem tractable. To obtain an optimal solution for the proposed CMRCMPSP using mathematical formulation is intractable and computational prohibitive. After over simplification, many authors have been able to handle up to 51 tasks (Rehm and Thiede , 2012). Formulation of the RCPSP model is as described below (Vanhoucke, 2013).

$$
f: \text { minimize } s_{n}
$$

Subject to:

$$
\begin{aligned}
& s_{i}+d_{i} \leq s_{j} \forall(i, j) \in A \\
& \sum_{i \in S(t)} r_{i, k} \leq a_{k} \forall k=1, \ldots, K ; t=1, \ldots, T \\
& s_{1}=0 \\
& s_{i} \in Z^{+} i=1, \ldots, n
\end{aligned}
$$

The objective function in equation (7) minimizes the entire project duration by minimizing the start time of the dummy end task. The constraint set given in equation (8) maintains the finish-start precedence relations with a time-lag of zero among the tasks. Equation (9) is used to model the availability of the renewable resources. It stipulates that the sum of the resource requirements of all activities in progress at time period $[\mathrm{t}-1, \mathrm{t}]$ is not allowed to exceed the availability of the renewable resource over the complete time horizon of the project T. Equation (10) forces the start task, and hence the project, to start at time zero. Equation (11) ensures that the task start times assume nonnegative integer values. The formulation of the MRCPSP model described by (Vanhoucke, 2013) is as follows: 


$$
\text { Minimize } \sum_{t=0}^{T} t * x_{n, 1, t}
$$

Subject to:

$$
\begin{aligned}
& \sum_{m \in M_{i}} \sum_{t=0}^{T} x_{i, m, t}=1 i \in I \\
& \sum_{m \in M_{i}} \sum_{t=0}^{T} t * x_{i, m, t}-\sum_{m \in M_{i}} \sum_{t=0}^{T}\left(t-d_{j, m}\right) x_{j, m, t} \leq 0 \\
& \sum_{i \in I} \sum_{m \in M_{i}} \sum_{t=0}^{T} r_{i, m, k} * x_{i, m, t} \leq A R_{t} \\
& x_{i, m, t} \in\{0,1\} \forall i, m, t
\end{aligned}
$$

The objective in equation (12) is to minimize the entire project duration by minimizing the start time of the end task. The constraint set given in equation (13) enforces that tasks can be executed only once during entire time horizon by selecting one mode out of the set of task modes. The constraint set given in equation (14) maintains the finish-start precedence relations with a time-lag of zero among the tasks. Equation (15) is used to model the limited availability of the renewable resources. It stipulates that the sum of the resource requirements of all tasks in progress during time period $[0, \mathrm{~T}]$ is not allowed to exceed the availability of the renewable resource over the complete time horizon of the project $\mathrm{T}$. Equation (16) ensures that $x_{i, m, t}$ variables assume integer values.

The mathematical formulations of RCPSP and MRCPSP fall in the class of Mixed-Integer Linear Programming models (MILP). Definition of MILP can be found in (Benichou et. at., 1971). The proposed CMRCMPSP model falls in the class of the Mixed-Integer Nonlinear Programming models. The problem is nonlinear if the objective function is nonlinear and/or the feasible region is determined by nonlinear 
constraints (MIT - Nonlinear, 2015). The CMRCMPSP model can be mathematically described as follows:

$$
f=\min \left(\left(\sum_{i \in I}\left(T C_{i}+\sum_{j \in J_{i}} T C_{i, j}\right)+T C R+T C S\right)\right.
$$

Subject to:

$$
\begin{aligned}
& \sum_{m \in M_{i, j}} x_{i, j, m, t}=1, \forall j \in J_{i}, \forall i \in I \text {, for all } t \in[0, T] \\
& \mathrm{x}_{\mathrm{i}, \mathrm{j}, \mathrm{m}, \mathrm{t}} * \mathrm{t} \geq \mathrm{st}_{\mathrm{i}, \mathrm{j}} \forall \mathrm{j} \in \mathrm{J}_{\mathrm{i}}, \forall \mathrm{i} \in \mathrm{I} \\
& \mathrm{ft}_{\mathrm{i}, \mathrm{j}} \geq \mathrm{st}_{\mathrm{i}, \mathrm{j}}+\mathrm{d}_{\mathrm{i}, \mathrm{j}, \mathrm{m}} \forall \mathrm{j} \in \mathrm{J}_{\mathrm{i}}, \forall \mathrm{i} \in \mathrm{I}, \forall \mathrm{m} \in \mathrm{M}_{\mathrm{i}, \mathrm{j}} \\
& r n_{(i, j, m, c)} * x_{i, j, m, t} \leq A R_{((i, j, m, c), t)}, \forall i \in I, \forall j \in J_{i}, \forall m \in M_{i, j}, \forall c \in C_{i, j, m}, \forall t \in[0, T] \\
& A R_{((i, j, m, c), t)} \leq \sum_{r \in R_{i, j, m, c}} z_{\left(R e_{(i, j, m, c, r)}, t\right)} * u_{\left(R e_{(i, j, m, c, r)}, t\right)}, \forall i \in I, \forall j \in J_{i}, \forall m \in M_{i, j}, \forall t \in[0, T] \\
& \sum_{i \in I} \sum_{j \in J_{i}} \sum_{m \in M_{i, j}} \sum_{c \in C_{i, j, m}} \sum_{r \in R_{i, j, m, c}} u_{\left(R e_{(i, j, m, c, r)}, t\right)} \leq 1,, \forall t \in[0, T] \\
& \sum_{m \in M_{i, b}} t * x_{i_{1}, b, m, t} \geq \sum_{m \in M_{i, a}}\left(t+d_{i_{1}, a, m}\right) * x_{i_{2}, a, m, t}, \forall(a, b) \in P, \forall i_{1}, i_{2} \in I, \\
& \text { for all } t \in[0, T] \\
& T C S_{(i, j, m, c, r)} \geq c S_{(s r)} * x_{i, j, m, t}, \forall i \in I, \forall j \in J_{i}, \forall m \in M_{i, j}, \forall c \in C_{i, j, m}, \forall r \in R_{i, j, m, c}, \\
& \forall \mathrm{sr} \in \mathrm{SRE}_{(i, j, m, c, r)}, \forall t \in[0, T] \\
& T C S \geq \sum_{i \in I} \sum_{j \in J_{i}} \sum_{m} \sum_{M_{i, j}} \sum_{c \in C_{i, j, m}} \operatorname{TCS}_{(i, j, m, c, r)} \text { for all } t \in[0, T]
\end{aligned}
$$




$$
\begin{aligned}
& A W_{((i, j, m, c), t)} \leq \sum_{y \in Y_{i, j, m, c}} z_{\left(N r_{(i, j, m, c, y)}, t\right)}, \forall i \in I, \forall j \in J_{i}, \forall m \in M_{i, j}, \forall c \in C_{i, j, m}, \forall t \in[0, T] \\
& w_{i, j, m, c} * x_{i, j, m, t} \leq A W_{((i, j, m, c), t)}, \forall i \in I, \forall j \in J_{i}, \forall m \in M_{i, j}, \forall c \in C_{i, j, m}, \forall t \in[0, T] \\
& A R_{((i, j, m, c), t+1)} \leq A R_{((i, j, m, c), t)}+A R D_{((i, j, m, c), t)}-M R D_{((i, j, m, c), t)}, \forall i \in I, \forall j \in J_{i}, \forall m \in M_{i, j}, \\
& \forall c \in C_{i, j, m}, \forall t \in[0, T] \\
& A W_{n r, t+1} \leq A W_{n r, t}+A W D_{n r, t}, \forall t \in[0, T] \\
& T C R \geq \sum_{n r \in N R} c w_{n r} T W_{n r}+\sum_{n r \in N R} c a w_{n r} T A W D_{n r}+\sum_{r e \in R E} c r_{r e} T R e_{r e}+\sum_{r e \in R E} c a r_{r e} T A R D_{r e} \\
& +\sum_{r e \in R E}{ } r o_{r e} T R O_{r e}+\sum_{r e \in R E} c r m_{r e} T R M_{r e} \text {, for all } t \in[0, T] \\
& T C_{i, j} \geq c_{i, j} *\left(\sum_{m \in M_{i, j}}\left(t+d_{i, j, m}\right) * x_{i, j, m, t}-d d_{i, j}\right), \forall i \in I, \forall j \in J_{i}, \text { for all } t \in[0, T] \\
& T C_{i} \geq c_{i} *\left(\sum_{m \in M_{i, N}}\left(t+d_{i, N, m}\right) * x_{i, N, m, t}-d d_{i}\right), \forall i \in I, \text { for all } t \in[0, T] \\
& x_{i, j, m, t} \in\{0,1\} \forall i \in I, \forall j \in J_{i}, \forall m \in M_{i, j}, \forall t \in[0, T] \\
& u_{\left(R e_{(i, j, m, c, r)}, t\right)} \in\{0,1\} \forall i \in I, \forall j \in J_{i}, \forall m \in M_{i, j}, \forall c \in C_{i, j, m}, \forall r \in R_{i, j, m, c} \forall t \in[0, T] \\
& Z_{\left(R e_{(i, j, m, c, r)}, t\right)} \in\{0,1\} \forall i \in I, \forall j \in J_{i}, \forall m \in M_{i, j}, \forall c \in C_{i, j, m}, \forall r \in R_{i, j, m, c}, \forall t \in[0, T], A R_{r e, t} \\
& \in Z^{+}, A W_{n r, t} Q, \forall r e \in R E, \forall n r \in N R
\end{aligned}
$$

Objective function given by equation (17) is to minimize the total tardiness over all projects. Constraint set defined by equation (18) ensures that all tasks are scheduled only once by selecting only one mode. Constraint set defined by equation (19) calculates start time for all tasks. Constraint set defined by equation (20) calculates finish time for all tasks. Constraint set defined by equation (21) imposes the maximum quantity of available renewable resource with capability $\mathrm{c}$ at time $\mathrm{t}$ for any project, task, and mode. Constraints set (22) calculate the maximum level of available renewable resources with capability 
$\mathrm{c}$ at time $\mathrm{t}$ according to the parameter $\mathrm{z}$ and binary variable $\mathrm{u}$. It is a nonlinear constraints. Constraint set (23) ensures that resources are not assigned for more than one project, task, mode, capability at the same time t. Constraint set (24) reflects the precedence relationships among the tasks of all projects. Constraint set (25) calculates the total cost associated of choosing a capability s of a renewable resources at time $\mathrm{t}$. Resource have multi-skills/capabilities, the first skill of the set (index 1) is the less costly, two is more costly, and so on so forth. This is a non-linear constraint, both are variables. Constraint set (26) calculates the total cost associated of choosing the capabilities of renewable resources for all time t. Constraint set (27) imposes the maximum level for the nonrenewable resource with capability c usage at time t over all projects. Constraint set (28) imposes the maximum quantity of available nonrenewable resources with capability c at time $\mathrm{t}$ for any project, task, and mode. Constraints set (29) calculate the maximum level of renewable resources with skill/capability $\mathrm{c}$ at time $\mathrm{t}$ according to the sum of the current quantity of renewable resources with skill/capability $\mathrm{c}$ at time $\mathrm{t}$ and renewable resources with skill/capability $\mathrm{c}$ added on time $\mathrm{t}$ minus the quantity of renewable resources with skill/capability $\mathrm{c}$ on maintenance. Constraints set (30) calculate the maximum level of nonrenewable resources $\mathrm{nr}$ at time $\mathrm{t}$ according to the sum of the current quantity of nonrenewable resources $\mathrm{nr}$ on hand and nonrenewable $\mathrm{nr}$ on order delivered on time t. Constraint set (31) calculates the total cost of the general renewable and nonrenewable resources. Constraint set (32) calculates the penalty cost due to tardiness values for each task. Constraint set (33) calculates the penalty cost due to tardiness values for each project. Constraint set (34) specifies the feasible ranges for the decision variables. Constraint set (35) specifies the feasible ranges for the decision variables. Constraint set (36) specifies the feasible ranges for the decision variables. 


\subsection{The Simulation Method}

None of the existing methods, exact or heuristic have developed an approach to solve the CMRCMPSP. Mathematical modelling of the CMRCMPSP is cumbersome. This research creates a discrete event based simulation to model and solve the CMRCMPSP. The solution method is called STREAM (Short-Term Resource Allocation and Management). In addition to simulation, STREAM has several other algorithms. Section 3.3.1 describes the Discrete Event Simulation (DES) algorithm. Section 3.3.2 describes the Multi-mode Scheduling and Queue Management (MSQM) algorithm. Section 3.3.3 describes the Priority Rules Management (PRM) algorithm. Section 3.3.4 describes the Worker Skills Management (WSM) algorithm. Section 3.3.5 describes the Task Duration Management (TDM) algorithm. Section 3.3.6 describes the Task Precedence Management (TPM) algorithm. The six algorithms were formulated and coded specifically to handle the CMRCMPSP model.

The solution method was primarily created with the goal to start tasks as early as they become eligible to be performed. If a given task is eligible to start and all required resources are available, the task will be started immediately. In order to solve resource conflicts among tasks during the simulation, STREAM uses multiple priority rules. STREAM attempts to require the least possible amount of resources to avoid waiting times for tasks. E.g., if 20 resources exist in the resource pool, STREAM may not assign all of them. STREAM assigns resources limited to 20 as long as they avoid resource conflicts among tasks. If more than 20 resources are required for tasks, some tasks will have to wait until required resources become available. Delays are automatically calculated, no over allocation in resources are permitted. 


\subsubsection{Discrete Event Simulation}

Simulation utilizes to a wide array of methods to mimic the behavior of complex systems [Kelton et al., 2002]. Simulation can be used to model a static or a dynamic environments. The focus of this work is on the dynamic systems. Figure 8 is an example of a continuous dynamic system. A weather system in which temperatures change overtime is an example of continuous dynamic system. A warehouse material arrival, material delivered, purchase orders, inventory levels are examples of a discrete dynamic system. Such systems follow a step function. Figure 9 shows a discrete dynamic system. Matloff [Matloff, 2008] provides an example where events decrease and increase the inventory levels, which are discrete variables. Chryssolouris [Chryssolouris , 2005] defines discrete-event simulation as following:

"Most simulation software programs model a manufacturing system as it evolves overtime by a representation in which the variables that track the systems state (the state variables) change instantaneously at separate points in time. These points in time, are the ones at which an event occurs, where an event is defined as an instantaneous occurrence that may change the state of the system. A model of this type is called a discrete event simulation model".

In shorts, for continuous systems, the state of the system changes continuously overtime, whereas for discrete dynamic systems, the state change occurs at discrete points in time. Program a discrete dynamic system is difficult because several tasks may have to be performed in parallel [Matloff, 2008].

"Simulation programming can often be difficult-difficult to write the code, and difficult to debug. The reason for this is that it really is a form of parallel programming, with many different tasks in progress simultaneously, and parallel programming can be challenging. For this reason, many people have tried to develop separate simulation languages, or at least simulation paradigms (i.e. programming styles) which enable to programmer to achieve clarity in simulation code. Special simulation languages have been invented in the past, notably SIMULA, which was invented in the 1960s and has significance today in that it was the language which invented the concept of object-oriented programming that is so popular today. However, the trend today is to develop simulation libraries which can be called from ordinary languages such as $C++$, instead of inventing entire new languages".

Figure 8 shows a system in which its behavior changes continuously overtime. It can be described by a function with a dependent variable $x(t)$ and independent variable $t$ [Cury, 2001]. In Figure 9 events are represented by letters $\alpha, \beta$ and $\lambda$. Each event affects the system differently depending upon its current state. 


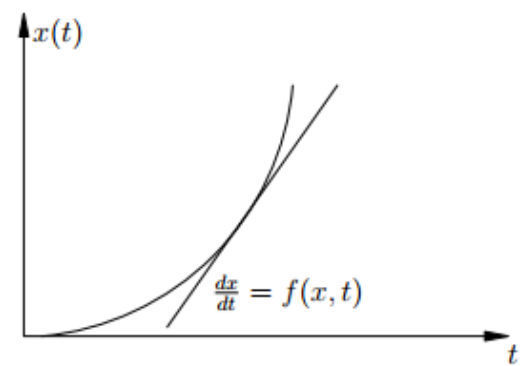

Figure 8:Typical behavior of Continuous Dynamic System

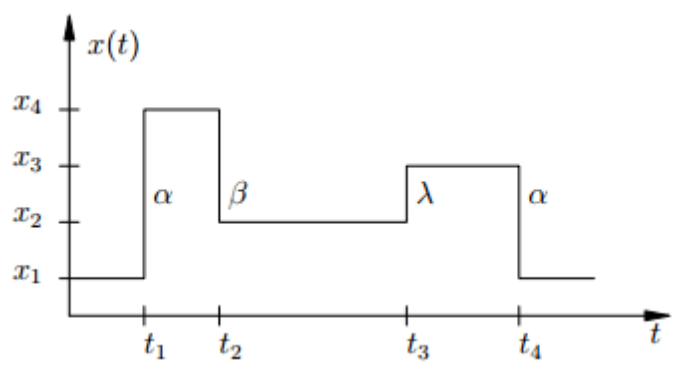

Figure 9: Typical behavior of Discrete Dynamic System

The simulation algorithm is based on discrete dynamic events and events can be inserted or deleted from the list of events during the simulation period. Events such as: start task, finish task, start resource maintenance, finish resource maintenance, can be used. Simulation period is the time horizon ranging from a date defined by the manager and date of the last task executed by simulation. Detailed description of general discrete dynamic event systems can be found in [Chryssolouris, 2005] and [Matloff, 2008].

The STREAM described in this work is based on a discrete dynamic event simulation using deterministic task duration. However, probabilistic task duration time ( $\mathrm{t}$ ) can be used by utilizing the approximated beta distribution where optimistic time is given by (a), pessimistic time is given by (b), and is the most likely time is given by $(\mathrm{m})$. Expected task duration time $(\mathrm{t})$ is approximated by the weighted average as $(a+4 m+b) / 6$ [Vanhoucke 2013]. STREAM prefers to use deterministic time values due to lack of reliable historical data, particularly when using multiple modes and multiple skills. Tasks can be rescheduled based on the current status of the system. The actual remaining time to finish tasks is also taken into account [1-Pinha et al. 2015, 2-Pinha et al. 2015]. Based on the nature of short-term project management, this research is concerned with discrete dynamic systems using deterministic task durations. Figure 10 provides a hierarchical representation of different classes of systems. Shaded boxes show scope of STREAM. 


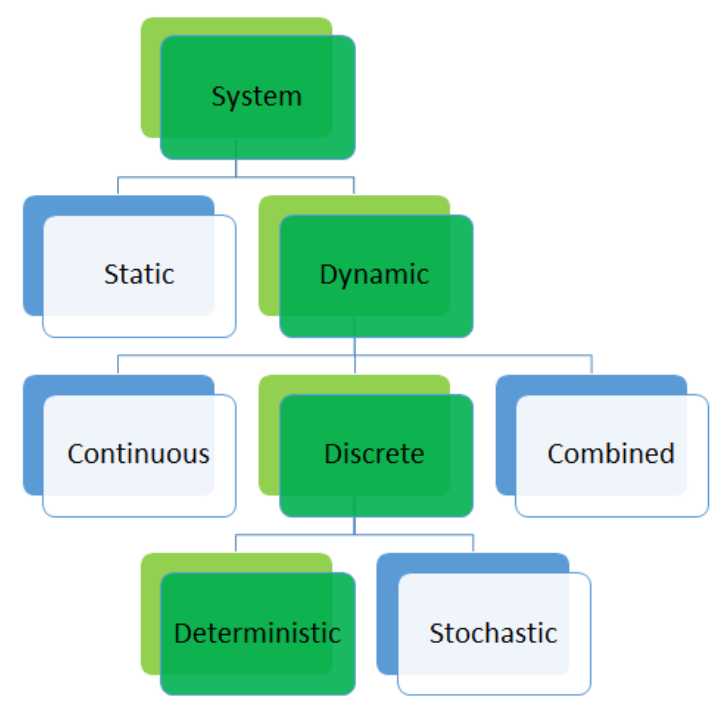

Figure 10: Overview Static and Dynamic System

The core algorithm of the Discrete Event Simulation (DES) is shown in Table 10. It generates outputs by scheduling tasks where the start date is specified by the manager. The simulation clock, a global variable, is initialized in the line 2. This variable controls how the clock moves in the simulated period. The project manager provides its initial value. Modes of eligible tasks join the queue (line 3). The "Run Queue" procedure (line 4) is activated to create events of finish tasks based on eligible modes. Also, priority rules are triggered by the "Run Queue" procedure in order to identify the winning mode in the queue when resource conflict exists. The "Run Queue" algorithm is described in Table 12. While events exist, the simulation keeps running until there are no more events (lines 5-25). Line 6 selects the most imminent event to occur. Line 7 verifies if the most imminent event is to finish a task, if so, the simulation clock is updated in line 8 and the finish time is registered in line 9. In line 10, resources are released. The task's successors may also be released if the number of predecessors executed by the simulator flagged as done is equal to the number of predecessors (line 14-15). In order to release a successor node, all predecessor nodes must be finished. Lines 16 to 18 register dates and modes are added 
to the queue. The executed event is removed from the list of events (line 22). Line 23 calls the procedure "Run Queue."

\begin{tabular}{|c|c|}
\hline & Table 10: Discrete Event Simulation (DES) Algorithm \\
\hline \multicolumn{2}{|r|}{ 1: procedure DES } \\
\hline 2: & Initialize simulation clock \\
\hline 3: & Modes of eligible tasks are sent to Queue \\
\hline 4: & Run Queue \\
\hline 5: & while exist events to be executed do \\
\hline $6:$ & Select the most imminent event to occur \\
\hline $7:$ & if Event type = Finish task then \\
\hline 8: & SimulationClock $\leftarrow$ ExecutionDate \\
\hline 9: & Finish $\leftarrow$ SimulationClock \\
\hline 10: & Release Resources \\
\hline 11: & Release Successors \\
\hline $12:$ & if Lagtime $=0$ then \\
\hline & for $S u c \leftarrow 1, N S u c_{i j}$ do \\
\hline $14:$ & N. Pred. done $\leftarrow$ N. Pred. done +1 \\
\hline 15: & if Number of Predecessors $=$ N. Pred. done then \\
\hline 16: & Eligible $\leftarrow$ True \\
\hline 17: & EligibleDate $\leftarrow$ SimulationClock \\
\hline 18: & Add modes to the queue \\
\hline 19: & end if \\
\hline 20: & end for \\
\hline 21: & end if \\
\hline 22: & Remove the executed event \\
\hline 23: & Run Queue \\
\hline 24: & end if \\
\hline & end while \\
\hline 26: & d procedure \\
\hline
\end{tabular}

\subsubsection{Multi-mode Scheduling and Queue Management}

This section presents the Multi-mode Scheduling and Queue Management (MSQM) algorithm. It establishes a concept of eligible modes, instead of eligible tasks as found in [Vanhoucke, 2013]. The Parallel Schedule Generation Scheme (PSGS) presented in [Vanhoucke, 2013] was expanded and named as Parallel Mode Schedule Generation Scheme (PMSGS). The PMSGS was presented in [Pinha et al, 2015]. Figure 11 shows a standard queue scheme commonly found in literature where tasks must select a queue to join and the resource must select a queue to server. Figure 12 also shows a standard 
representation of a queue found in the literature where tasks must select one resource out many [Khoshnevis, 1994].
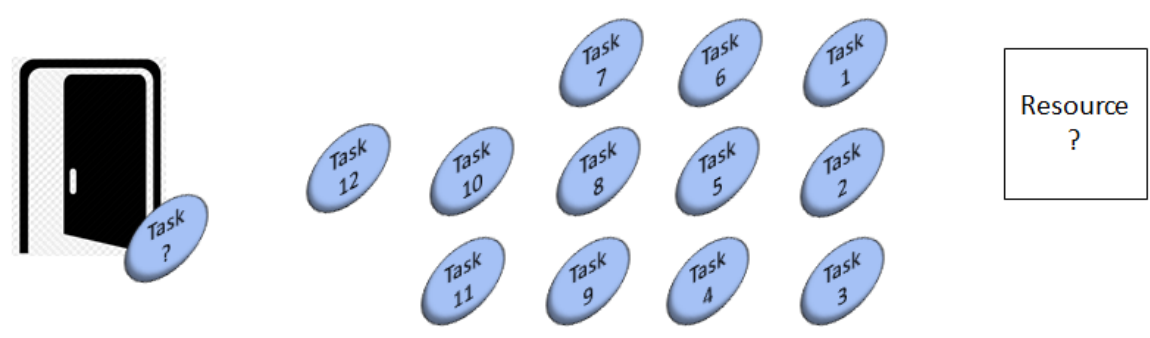

Figure 11: Selection of a queue to join and selection of a queue to serve
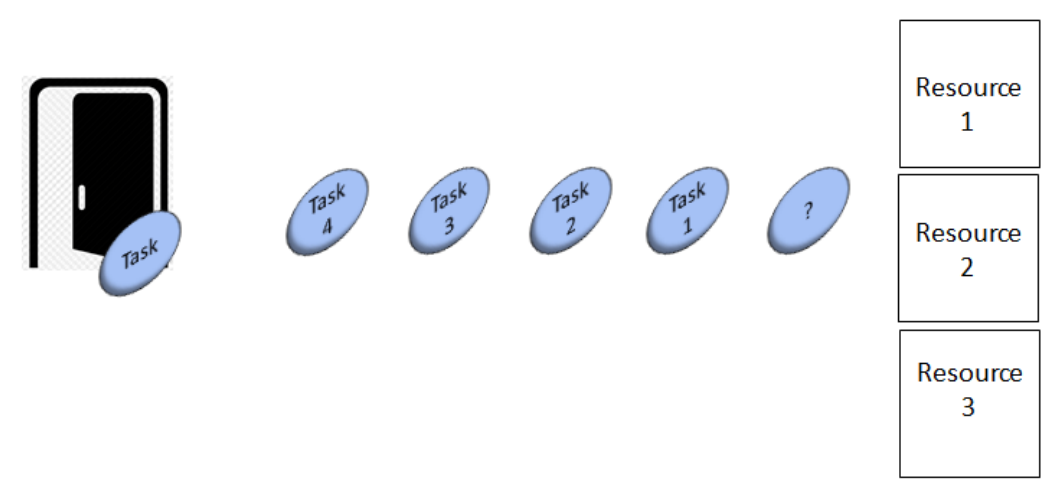

Figure 12: Selection from one out of several servers

It is difficult to organize queues when a single task has 896 different options and each resource has its own rules. The traditional queue management schemes do not support and cannot be applied to queue problem in CMRCMPSP. Table 11 shows resource requirements for Tasks 2 and 3 of Project 1. Figure 13 recalls the example 1 described in Section 3.1. 


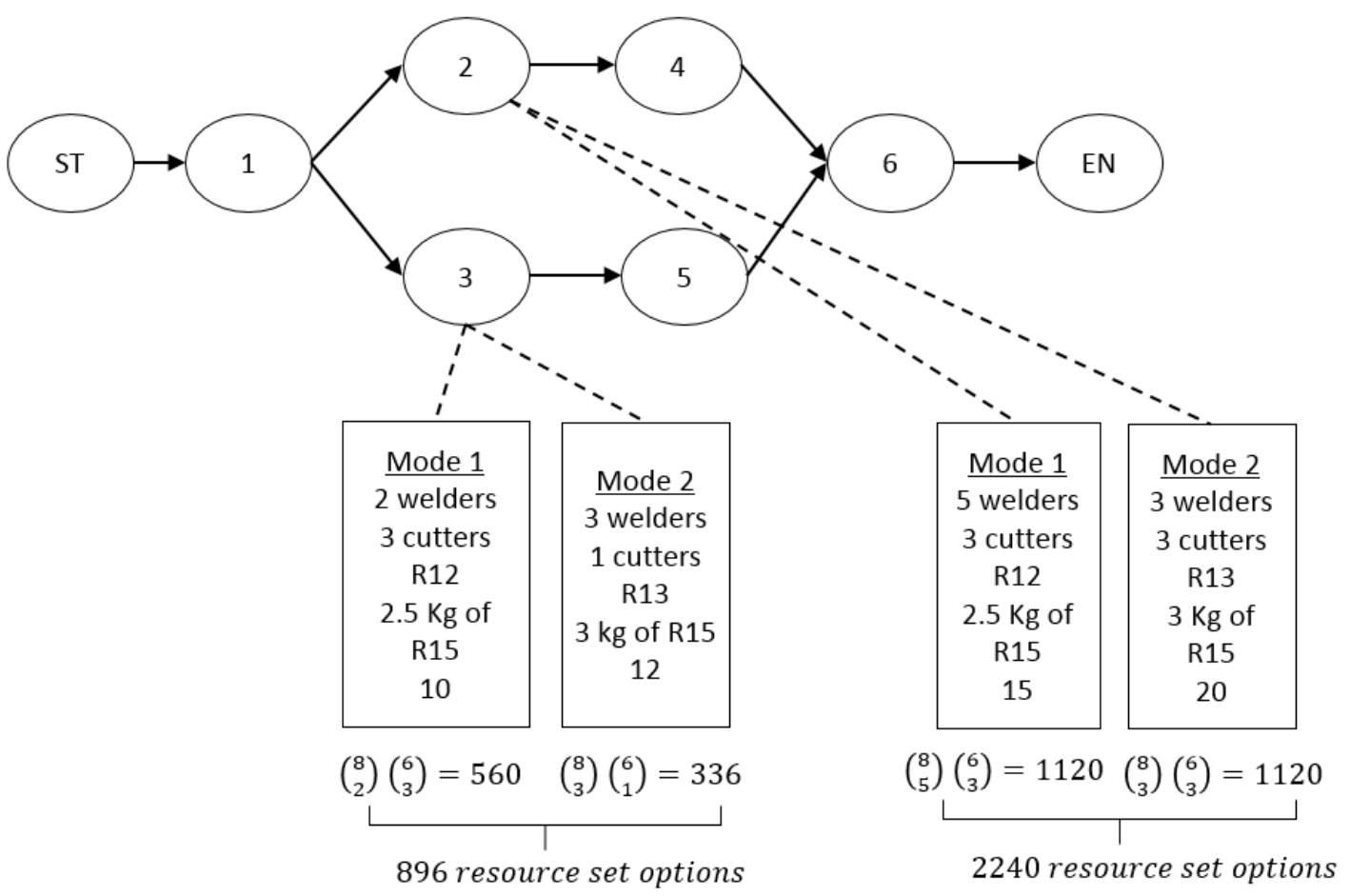

Figure 13: Task on node for the CMRCMPSP

Table 11: Mode description for task 2 and task 3 of project 1.

\begin{tabular}{|c|c|c|c|}
\hline Task & Mode & Resource Requirements & Duration \\
\hline \multirow{2}{*}{2} & 1 & 2 welders out of 8, 3 cutters out of 6, R12 and 2.5 Kg of R15 & 10 \\
\cline { 2 - 4 } & 2 & 3 welders out of 8,1 cutter out of $6, \mathrm{R} 13$ and $3 \mathrm{Kg}$ of R15 & 12 \\
\hline \multirow{2}{*}{3} & 1 & 5 welders out of 8,3 cutters out of $6, \mathrm{R} 12$ and $2.5 \mathrm{Kg}$ of R15 & 15 \\
\cline { 2 - 4 } & 2 & 3 welders out of 8,3 cutters out of $6, \mathrm{R} 13$ and $3 \mathrm{Kg}$ of R15 & 20 \\
\hline
\end{tabular}

Figure 14 shows the complexity of the queue management for the CMRCMPSP. It only shows the 4 eligible modes associated with the eligible Tasks 2 and 3, of Project 1. The four oval shapes represent modes. The white oval shapes are modes related to Task 3, of Project 1 and the black ones are associated with Task 2, of Project 1. For simplification purposes, only Modes 1 of both tasks have association with their resource combinations. 


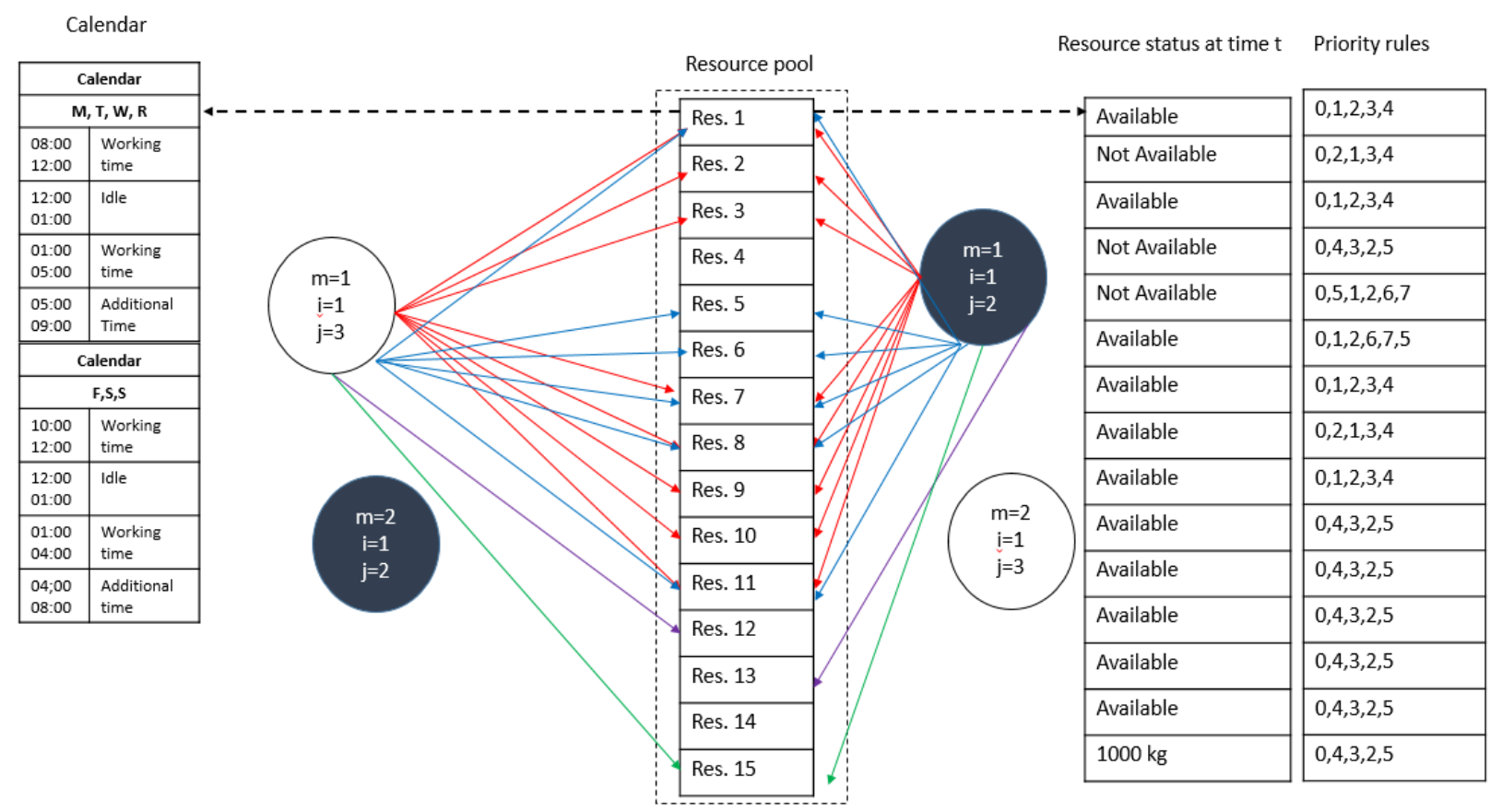

Figure 14: CMRCMPSP Queue Schema

As can be seen from the Figure 14 several links exist between modes and corresponding resources. These links are the resource requirements for each mode. Unavailability of a resource out of several resources can hold a task for being started. Resources can be renewable or nonrenewable. The queue management approach utilized by STREAM to solve the CMRCMPSP is as follows:

1. Break individual task into their respective modes.

2. There is no predefined and organized queue.

a. There is no benefit to organizing a queue ahead of time because each resource has its own priority rules. It is difficult to organize a queue if the same mode requires different resources, and their rules are in conflict to each other.

b. Eligible modes from eligible tasks are listed as possible candidate for execution.

c. The decision of which mode wins among eligible modes is performed at each decision point in time. 
3. Verify if all the required resources for the first mode of a given task are available.

a. If so, verify if all the required resources for the first mode of given different task are available.

b. If no common resources exist between these two modes, mode 1 of the first task is a tentative winner and further comparison is performed with another mode.

c. If a common resource exists, a priority rule defines which mode wins for the first pair comparison

d. If the modes are still tied up, a second priority rule is used, and so forth. The comparison is performed while modes exist. In the end, only one mode wins

In order to deal with the queue management complexity in CMRCMPSP a new approach was utilized. The PMSGS selects and ranks eligible modes instead of eligible tasks at each decision point time through the simulation period. Eligible modes are those that are connected to eligible tasks. Different tasks can have modes that require a same set of resources, at the same time. Priority rules, as described in section 3.4.3, based on heuristics corresponding to a given application were developed to handle such conflicts. A separate procedure named "Run Queue" was implemented to control priorities, dynamically. If one priority rule is not enough to break a tie among modes, the second priority rule is used. Once a mode has been selected, other modes related to that task are dropped from the mode queue. This process is performed for all eligible modes simultaneously, at a given decision point time in PMSGS.

Figure 15 shows a hypothetical PMSGS scenario for Tasks 2 and 3. Due to the precedence order, Task 1 must be finished before Tasks 2 and 3. Assuming that Task 1 has been completed, Tasks 2 , and 3 become eligible to start and therefore need to be scheduled at the given simulation clock time. Their modes, therefore, are automatically eligible and if resource conflict exists, the priority rules may have to be triggered. Notations $\mathrm{i}, \mathrm{j}$, and $\mathrm{m}$ are index for projects, tasks and modes, respectively. 


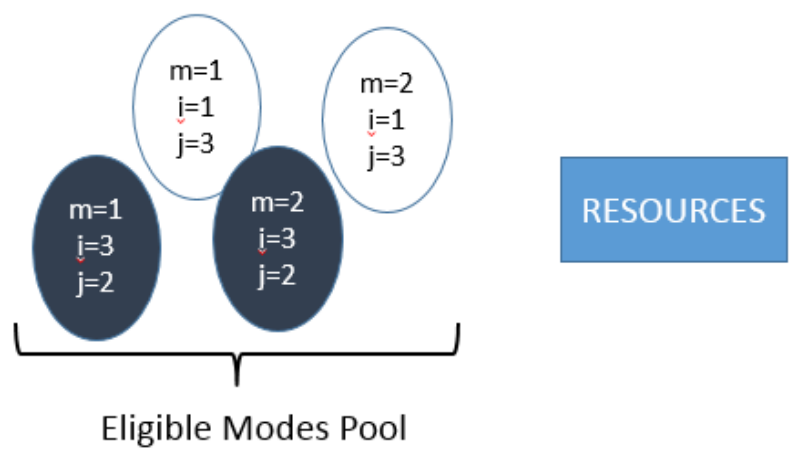

Figure 15: Hypothetical Scenario

Table 12 summarizes the Run Queue Algorithm. This procedure verifies if required resources are available for current modes in the queue and which mode currently has the highest priority, if resource conflict exists. The term current priority is used because modes are not sorted into the queue to be scheduled. When this procedure is called at a given simulation clock, the last priorities of modes may have nothing do to with the current situation. One mode which could have been in the second priority at the last decision time, may have the last priority at the current decision time. Modes which might have been added to the queue at to the current simulation clock may completely change priorities in the queue. Therefore, modes in queue change dynamically, according to their priorities at each decision point. In other words, whenever the procedure "Run Queue" is called, priorities are set dynamically for a given mode.

Line 2 of the Run Queue algorithm verifies if number of current modes in the queue is equal to one, if so, there is no competition among modes and then resources are required if they are available (Line 3). If resources are available, the algorithm registers the start time, requires resources and remove mode of queue (lines 4 to 6). If there is more than one mode in the queue, the algorithm goes over all the current modes (line 8). Each mode in the queue has an index $q 1$. Line 10 verifies if resources required for the mode with index $q 1$ are available, if so, the algorithm verifies for all other modes with index different than $q 1$, in this case $q 2$. From lines 12 to 24 , the algorithm searches for the highest priority mode. If modes require common resources, the search is performed by selecting two modes at time, and one is 
selected based on the priority rule. The final winner is the one which has the highest priority over all the modes at a given decision time. Lines 25 to 33, once a winner has been found, the start time is registered, resources are required, the event of finish task is added to the list of events and modes are removed from the queue. Line 25 checks if resource is not available for the competing mode with index $q 2$, at this decision time or if no common resource exists between mode with index $q 1$ and $q 2$. If one of this condition is false, it means no competition exists among the two modes and mode with index $q 1$ wins. While there are modes in queue which require available resources, event of finish task is added to the list of events. Events are added through "Run Queue" procedure in lines 4 and 26. The variable $q$ is the index for current modes in the queue ranging from $1<q<N M Q_{t} . N M Q_{t}$ is the number of current modes in the queue at time t; and $M Q_{q t}$ is the mode with index $q$ in the queue at time $t$.

Table 13 describes the start time recording procedure used by the Run Queue algorithm. It aims to find when a given task is able to start based on the current simulation clock (line 2). Also projected Finish $_{\text {event }}$ is calculated (line 4 or 6). A variable Key is assigned to be the concatenated code between the date of the projected Finish event $_{\text {and }}$ ane Task Index (line 8). Line 9 the event of Finish Task is added to the list of events. 


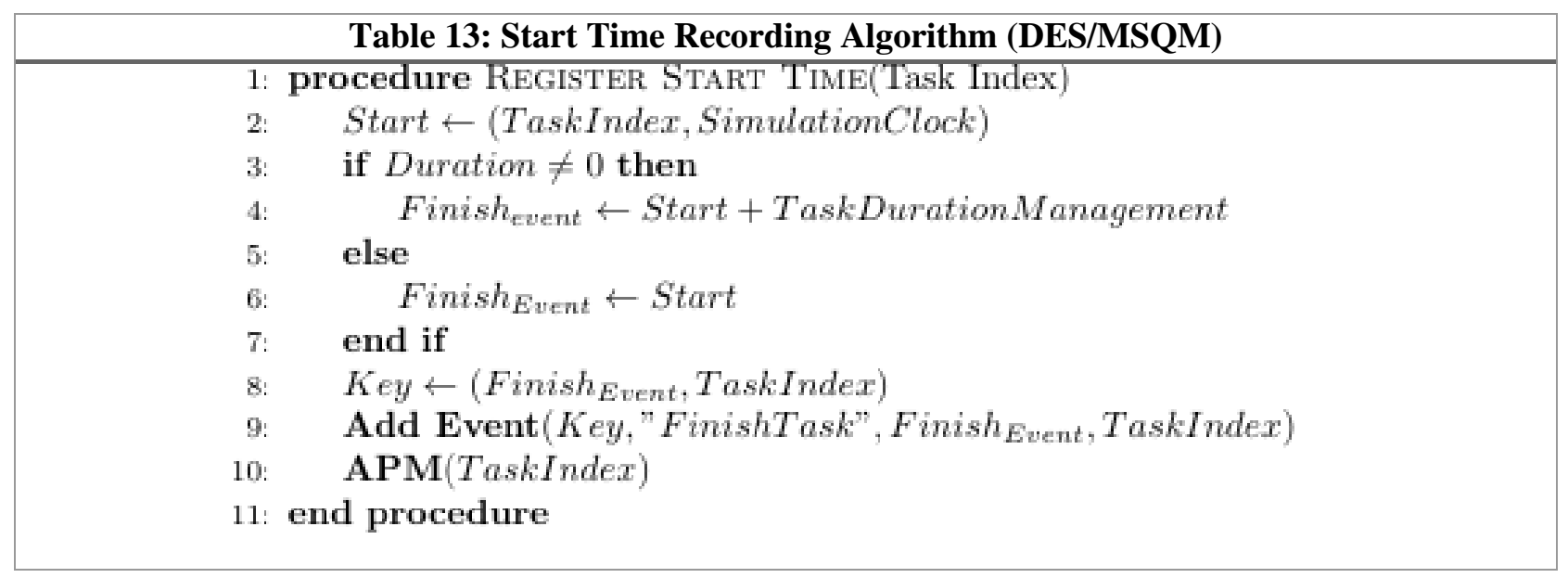

\subsubsection{Priority Rules Management}

Vanhoucke [Vanhoucke, 2013] specified four types of priority rules: a) Task based priority rules, b) Network based priority rules, c) Critical path based priority rules, and d) Resource based priority rules. Multi-modes with multi-constrained resources for each task require complex management of queues. Resources availability ultimately decides which task will have priority.

A mode, in this research, has information regarding correlated tasks. There is traceability along projects, tasks, modes, and resource requirements. Therefore, this method will work, regardless of the rule, for all four of types of priorities. This research uses composite priority rules of the four rule classes mentioned by Vanhoucke [Vanhoucke, 2013]. However, instead of using a weighted combination, we propose a class called "Independent Composite Priority Rules". The rules are specified for each resource, or set of resources, in priority order, without any weight. Each resource has the flexibility to have its own rules, e.g., rule for Resource 1 (welding resource for example) do not apply to Resource 2 (painting worker) and vice-versa. Indeed, it is common practice that each unit runs its own operations.

If the priority rules for Resource 1 are: 1) Customer deadline, 2) Shortest processing time, 3) Immediate successor need, and 4) Earliest completion time, and if two modes of different tasks are in the queue requiring Resource 1, then the priority rule 1 is used to resolve the issue. If the tasks have the same customer deadline, then the second rule is applied and so on so forth. 
Figure 16 shows a schema of the four modes in the queue requiring a different set of resources. This schema based on example 1 shows only one combination out of several for Tasks 2 and 3 with their respective Mode 1 (Table 8 and Figure 6). The resource request process is made every time a resource is released. The status of resources is taken into consideration and once a resource conflict exists, priority rules are called to determine which mode wins. Notice that each resource may have its own priority rule list. A single combination for performing Task 2, Mode1, and Task 3, Mode 1 can be described as follows:

Task 2, Mode $1=\{$ R1, R2, (welders), R5, R6, R11 (cutters), R12, R15 $\}$.

Task 3, Mode $1=\{$ R3, R7, R8, R9, R10 (welders), R5, R6, R11 (cutters), R12, R15 $\}$.
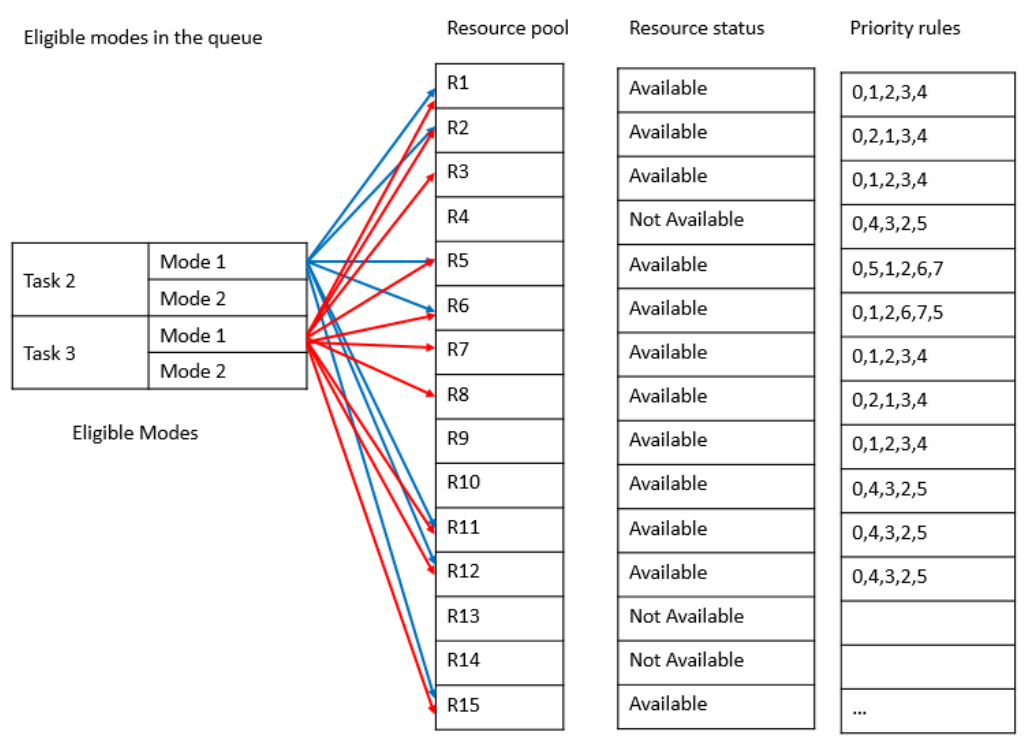

\begin{tabular}{|l|l|}
\hline Rule & Description \\
\hline 0 & Priority \\
\hline 1 & Earliest due date \\
\hline 2 & Fastest duration \\
\hline 3 & Lowest cost \\
\hline 4 & Highest profit \\
\hline 5 & Same project \\
\hline 6 & Remaining time \\
\hline 7 & Time in Queue \\
\hline
\end{tabular}

Figure 16: Modes x Resource pool

Assuming that only Modes 1 and 2 for Tasks 2 and 3 are in the queue at given decision point in time. A Venn diagram depicted in Figure 17 shows which resources are in conflict. Resource R5, R6 and R11 are required by both modes. Therefore, there is a resource conflict. Notice, all of these resources are available. They have been previously identified as available, the question at this point is: Which one of 
the two modes should be selected? Priority rules are called and applied only for resources R5, R6, and R11 to solve conflict between Modes 1 of Tasks 2 and 3.

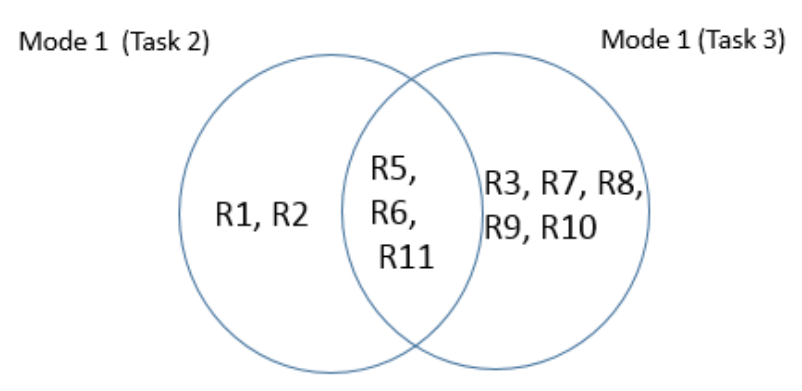

Figure 17: Venn diagram for resource conflicts

The Priority Rules Management (PRM) algorithm is shown in Table 14. This algorithm has 3 parameters: Task 1 index, Task 2 index and Resource Index. At each iteration, two modes of different tasks are utilized by the algorithm. This information comes from Task 1 index and Task 2 Index. The selected mode for each task was previously found by the Run Queue Algorithm. The third parameter "Resource Index" provides the index of a common resource along the modes, in other words, the conflicting resources. This procedure returns one temporary winning mode named "PreliminaryWinner". The tentative winning mode becomes a definitive winning mode once all modes in the queue have been verified. Based on the Resource Index, its priority rules number is known. Line 2 to line 19 perform a loop is performed in order to run all possible priority rules (from rule 1 to $N P R_{n r}$ ) to break ties among two modes, if necessary. Rule with index 1 is the highest priority rule, rule 2 is more important than 3 , and so forth. In line 3 assuming that a given resource has the rule "Earliest Deadline", there will be a comparison between the deadline of Task 1 index and Task 2 index, the task which presents the earliest deadline will be the "PreliminaryWinner" (Line 4). The same logic follows from lines 5 to 13. Line 14 checks if the two tasks are still tied (Preliminary Winner $=-1$ ) and also checks if all possible priority rules have been used. If so, the PreliminaryWinner is equal to Task 1 Index because all effort has been done and they are still tied. If such condition occurs, first index is chosen. Line 16, on the other hand, checks if any Priority 
rule was enough to break a tie (PreliminaryWinner $\neq-1$ ). If so, the loop ends because the resource conflict has been solved for at least one priority rule. $N P R_{z}$ is the number of priority rules for resource $z$, and $p r$ is the rule index with range $1<p r<N P R_{z}$.

\section{Table 14: Priority Rules Management (PRM) Algorithm}

1: procedure PRM(Task 1 index, Task 2 Index, Resource Index=nr)

2: $\quad$ for $p r \leftarrow 1, N P R_{n r}$ do

3: $\quad$ if ("Earliest Deadline" $=$ Rule $_{p r}$ ) then

4: $\quad$ PreliminaryWinner $\leftarrow$ winner from Deadline

5: else if ("Fastest Duration" $=R u l e_{p r}$ ) then

6: $\quad$ PreliminaryWinnerr $\leftarrow$ winner from Fastest Duration

7: $\quad$ else if (Rule $3=$ Rule $_{p r}$ ) then

8: $\quad$ PreliminaryWinner $\leftarrow$ winner from Rule 3

9: $\quad$ else if $(. .$.$) then$

10: $\quad$ PreliminaryWinner $\leftarrow$ winner from $\ldots$

11: $\quad$ else if (Rule $\mathrm{n}=$ Rule $_{p r}$ ) then

12: $\quad$ PreliminaryWinner $\leftarrow$ winner from Rule $\mathbf{n}$

13: $\quad$ end if

14: $\quad$ if (PreliminaryWinner $=-1$ ) and $\mathrm{pr}=N P R_{z}$ then

15: PreliminaryWinner $\leftarrow$ Task 1 Index

16: $\quad$ else if (PreliminaryWinner $\neq-1$ ) then

17: $\quad$ Exit For

18: $\quad$ end if

19: end for

20: end procedure 


\subsubsection{Worker Skills Management}

STREAM does not use a pre-determined approach to project management. Instead, it is based on the notion of dynamically allocating resources. The flexibility in resource allocation is extended to worker skills to reduce project cost and duration. Figure 18 shows the Venn diagram for skills for example 1, as described in the Section 3.1.

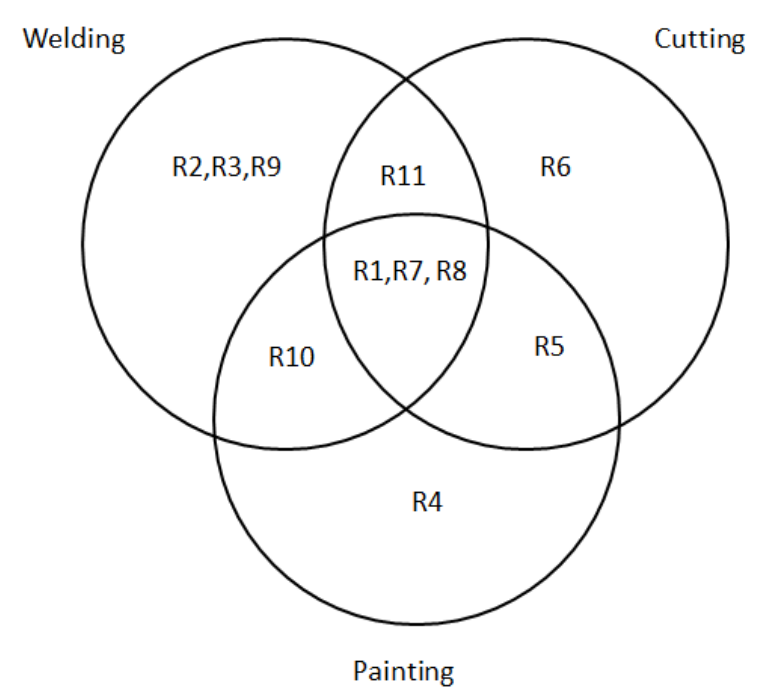

Figure 18: Venn diagram for skilled resources

One of the gaps identified in the literature is modelling of production capacity. If the capacity is not well defined, the results will not be accurate. Several authors have raised this issue, but very little information is available on how to address this problem. Traditional project scheduling is limited to single-skilled resource assumption. This does not represent the real-world practice where workers have multiple skills and are assigned to perform tasks where they are not primarily specialized [Wongwai and Malaikrisanachalee 2011]. However, some limitations regarding multi-skilled workers still exist: different work hours for workers, cost assessment, and overtimes, etc. STREAM's approach differs from Wongwai and Malaikrisanachalee (2011) by providing qualitative rank for different skills, such as excellent, good, satisfactory, and not applicable for each resource as introduced in section 3.1. 
In STREAM, if a task requires a certain skill, say welding, it checks the available resource that have welding as their primary skill. If none is found, it checks for resources that have welding as their secondary skill, if found, it assigns that resource to the task. The resource could have painting as the primary skill, cutiing and welding as secondary skill. When a worker is assigned to perform the task using one of his many skills, then his/her other skills are not available to other task. Typical project management tools assume resources to have a single skill, resulting in schedule delay and higher cost.

In STREAM a method called Worker Skill Management (WSM) was created. WSM has two parts, i) Available Resource and Skills Management (ARSM) and ii) Resource and Skills Requirement Management (RSRM). Table 15 describes the ARSM algorithm. Table 16 describes the RSRM algorithm. The ARSM verifies if resources are available with required skills at a given decision point in time. The RSRM actually requires and assigns the resources based on the skill required.

The ARSM has two parameters, SkillRequiredSelectedMode and NumberOfResources for each required skill. The algorithm starts with a loop which runs with a variable $s$ equal to 1 , to the number of skills $(N S)$. Line 3 checks if a skill required for a selected mode is equal to a general $s k i l l_{s}$. If yes, it runs

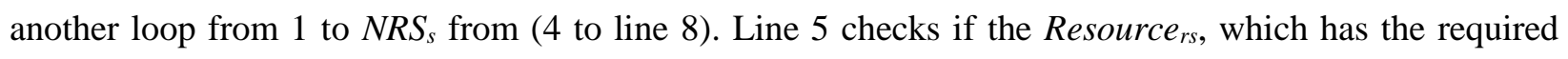
skill, is available. If so, line 6 the variable Counter is added of one. Line 11 verifies if the variable Counter is greater or equal to the number of resources required by a given task, if so, that means resources are available (line 12), if not, resource are not available (line 14). NS is the number of skills; $s$ is the skill index with range $1 \leq s \leq N S$; and $N R S_{s}$ is the number of resources with $s k i l l_{s} ; r s$ is the resource index with range

$1 \leq r s \leq N R S_{s}$ 


\begin{tabular}{|c|}
\hline Table 15: Available Resource and Skills Management (ARSM) algorithm/WSM \\
\hline 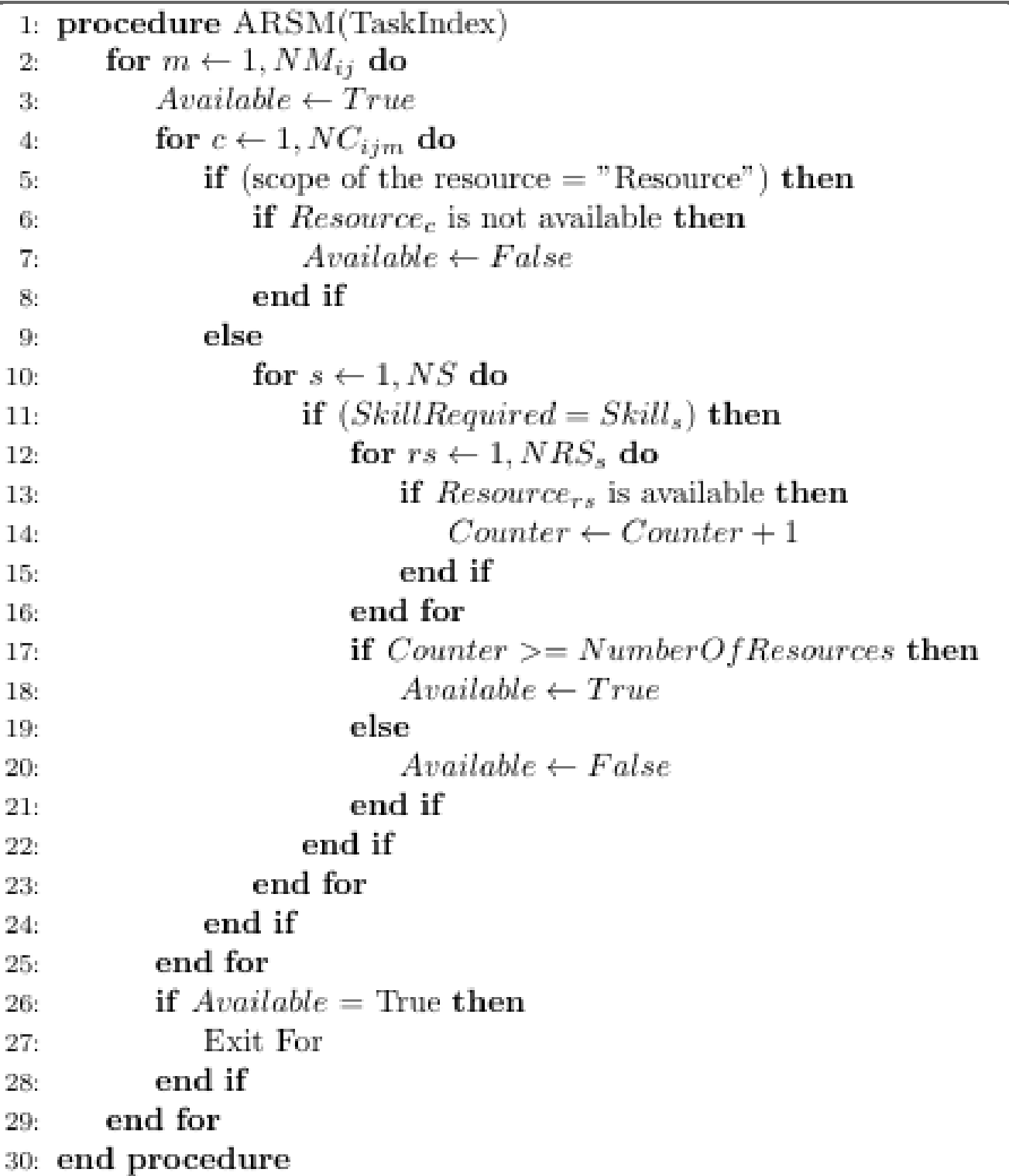 \\
\hline
\end{tabular}

The RSRM has a single parameter, Task Index. The algorithm initializes a variable $k$ which goes from 1 to the number of resource for the mode selected $\left(N C_{i j m}\right)$. It handles resources and skills associated with a given resource. Line 3 checks if the scope of the select resource is "Resource". It can be either a resource or a skill. If it is "Resource", the resource for the select mode becomes occupied (line 4), else, the scope is "Skill" and then from lines 6 to 19, a loop of skills is performed in order to find the skill required for the mode selected (Line 7 - If a skill required for a selected mode is equal to a general $s k i l l_{s}$ ). 
If yes, run another loop from 1 to $N R S_{s}$ from line 8 to line 17. Line 9 checks if the variable Counter is less or equal to $N R S_{s}$. If so, line 10 verifies if Resource $_{r s}$ is available, if it is available then Resource $_{r s}$ becomes unavailable in line 11 and the variable Counter goes up by one in line 12 . If the variable Counter is greater than the number of resources required by a given task, the loop is finished (line 15). Variable Counter being greater than the number of resources required means that the number of required resources for a given skills to execute a task has been satisfied. $N S$ is the number of skills; $N R_{i j m}$ is the number of resources for project $i$, task $j$, mode $m ; N R S_{s}$ is the number of resources with skill $s$; $r s$ is the resource index with range $1 \leq r s \leq N R S_{s .} ; s$ is the skill index with range $1 \leq s \leq N S$; and $k$ is the resource index with 1 $\leq k \leq N R_{i j m}$

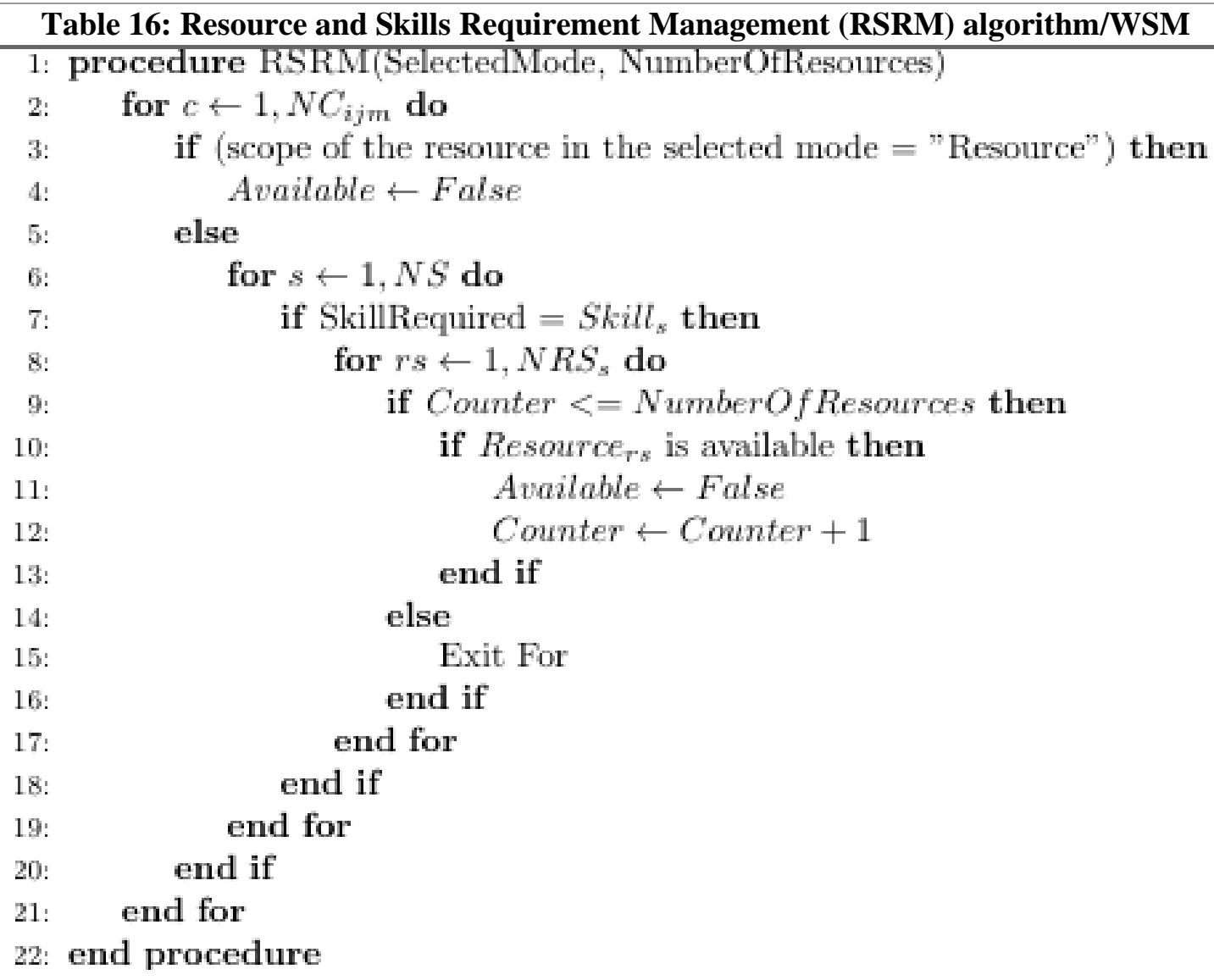




\subsubsection{Task Duration Management}

STREAM allows each resource to have several time intervals during a given day. That means a single resource can have different daily calendar, that is, a given skilled worker can work from 8:00 to 17:00 on Mondays, from 9:00 to 16:00 on Tuesdays, and so on. A "shift" is defined as eight hour work day and the time interval is further classified as "Regular", "Idle," and "Overtime." Time intervals classification can vary for each work day, that is, Monday could have 8:00 to 12:00 (Regular), 12:00 to 13:00 (Idle), and 13:00 to 17:00 (Regular). Tuesday's time classification could be 9:00 to 9:30 (idle), 9:30 to 12:30 (regular), 12:30 to 13:00 (idle), and 13:00 to 16:00 (regular), etc. Time intervals would vary according to project needs. Such an approach allows unlimited flexibility for time classification. The longest calculated duration along all resources will be the actual time taken to execute a given task using a given mode. Since each resource has its own calendar, the slowest resource or the most constrained one will result in the actual task duration. Real world projects have several calendar constraints, adding further complexity to the model. Table 17 shows the calendar for the fourteen renewable resources of example 1 presented in section 3.1. Table 18 shows a work day for resource R1 as an example.

Table 17: Resource Calendar

\begin{tabular}{|c|c|l|}
\hline Resource & Number of days/week & \multicolumn{1}{|c|}{ Days } \\
\hline R1 & 5 & M, W, T, Th., F \\
\hline R2 & 5 & M, W, T, Th., F \\
\hline R3 & 5 & M, W, T, Th., F \\
\hline R4 & 6 & M, W, T, Th., F, S \\
\hline R5 & 4 & M, W, T, Th. \\
\hline R6 & 7 & M, W, T, Th., F, S, Su. \\
\hline R7 & 6 & M, W, T, Th., F, S \\
\hline R8 & 5 & M, W, T, Th., F \\
\hline R9 & 5 & M, W, T, Th., F \\
\hline R10 & 5 & M, W, T, Th., F \\
\hline R11 & 5 & M, W, T, Th., F \\
\hline R12 & 5 & M, W, T, Th., F \\
\hline R13 & 5 & M, W, T, Th., F \\
\hline R14 & 5 & M, W, T, Th., F \\
\hline
\end{tabular}




\begin{tabular}{|c|c|c|l|}
\hline \multicolumn{4}{|c|}{ Table 18: Work Day Classification for Resource R1 on Monday } \\
\hline TIP & From & To & \multicolumn{1}{|c|}{ Description } \\
\hline 1 & $00: 00$ & $8: 00$ & Idle (non-working time) \\
\hline 2 & $08: 00$ & $12: 00$ & Regular (working time) \\
\hline 3 & $12: 00$ & $13: 00$ & Idle (lunch time) \\
\hline 4 & $13: 00$ & $18: 00$ & Regular (working time) \\
\hline 5 & $18: 00$ & $24: 00$ & Overtime (working time with additional pay) \\
\hline
\end{tabular}

Project management tools primarily focus on production times. Non-production times are extremely complicated and cumbersome to handle ahead of time, because it requires an exhaustive analysis of resource queues. It is difficult to estimate how long a task will wait for its resources once it is eligible to start. Traditional approaches do not account for idle time. Calendar constraints have significant impact on project duration time. For instance, if the duration of a given task requires 24 hours using a set of resources, it does not mean that this task can be carried out in three days of 8 hours shift. Depending upon resource availability, the task could take 48 hours, 96 hours, and so forth. The duration will depend upon break constraints and also the multiple resource calendars involved with the task

Table 19 summarizes the Task Duration Management (TDM) algorithm. It calculates the actual duration of tasks, taking into account constraints such as, maintenance times, lunch time, and other idle times. These types of calendar constraints exist for all projects and they add complexity to the simulation model. Additional times such as overtimes are considered and it can also affect tasks duration. Therefore, the simulation, through TDM algorithm, will calculate the actual finish time considering all the complexities mentioned above. $N R_{i j m}$ is the number of resources for project $i$, task $j$, and mode $m ; N I D R_{z}$ is the number of time interval partitions during a day for resource $z$; and $\mathrm{k}$ Resource index, $1 \leq k \leq N R_{i j m}$.

The TDM algorithm has three parameters, Task Index, Duration, and Current Clock. Duration is the production time provided by managers and Current Clock takes the information of the current value of the global variable Simulation Clock. Line 2 assigns the week day of the current clock to variable Day, for instance if the current clock is (2/26/2016 1:30 PM), variable Day becomes equal to 5 because 2/26/2016 12:00 is Thursday (Sunday $=1$, Monday $=2$, Tuesday $=3$, Wednesday $=4$, Thursday $=5$, Friday $=6$ and 
Saturday $=7$ ). From line 3 to 26 there is a loop that runs from 1 to number of required resources of the selected mode $\left(N R_{i j m}\right)$. Line 4 Remaining time is assigned to be equal to Duration. When Remaining reaches 0 , it means the task could have been executed in its total time across any calendar constraint. Line 5 calls the Availability Verification Management $(A V M)$ procedure.

The AVM algorithm is shown in Table 20. Its goal is to figure out what is the time interval during the day for the Current Clock and it also adds possible idle times accordingly to variable Duration. Since each time interval has its own start time and end time, this procedure finds the time interval partition (TIP) and if it is a "Regular", "Idle", or "Additional". Line 29 in the AVM algorithm checks if the current TIP is "Regular", if so, verification if any maintenance or other idle times will exist is needed. In line 30, the variable Add Idle Time controls how much idle time exists through the TIP. In line 31, the variable Remaining is decreased by the time TIP value. If the TIP is "Idle" (line 32) no changes is made for the variable Remaining, only for Add Idle Time (line 33). If TIP is "Additional" (Line 34) then variable Remaining is decreased by the amount of time the TIP allows in additional time (line 35). "Authorized Additional Time" is a procedure which finds if additional time is authorized. Line 36, Add Idle Time is added by the current TIP end time minus any authorized additional time. Line 38 checks if Remaining is equal to 0 , if so, exit procedure. 
Table 19: Task Duration Management (TDM) algorithm

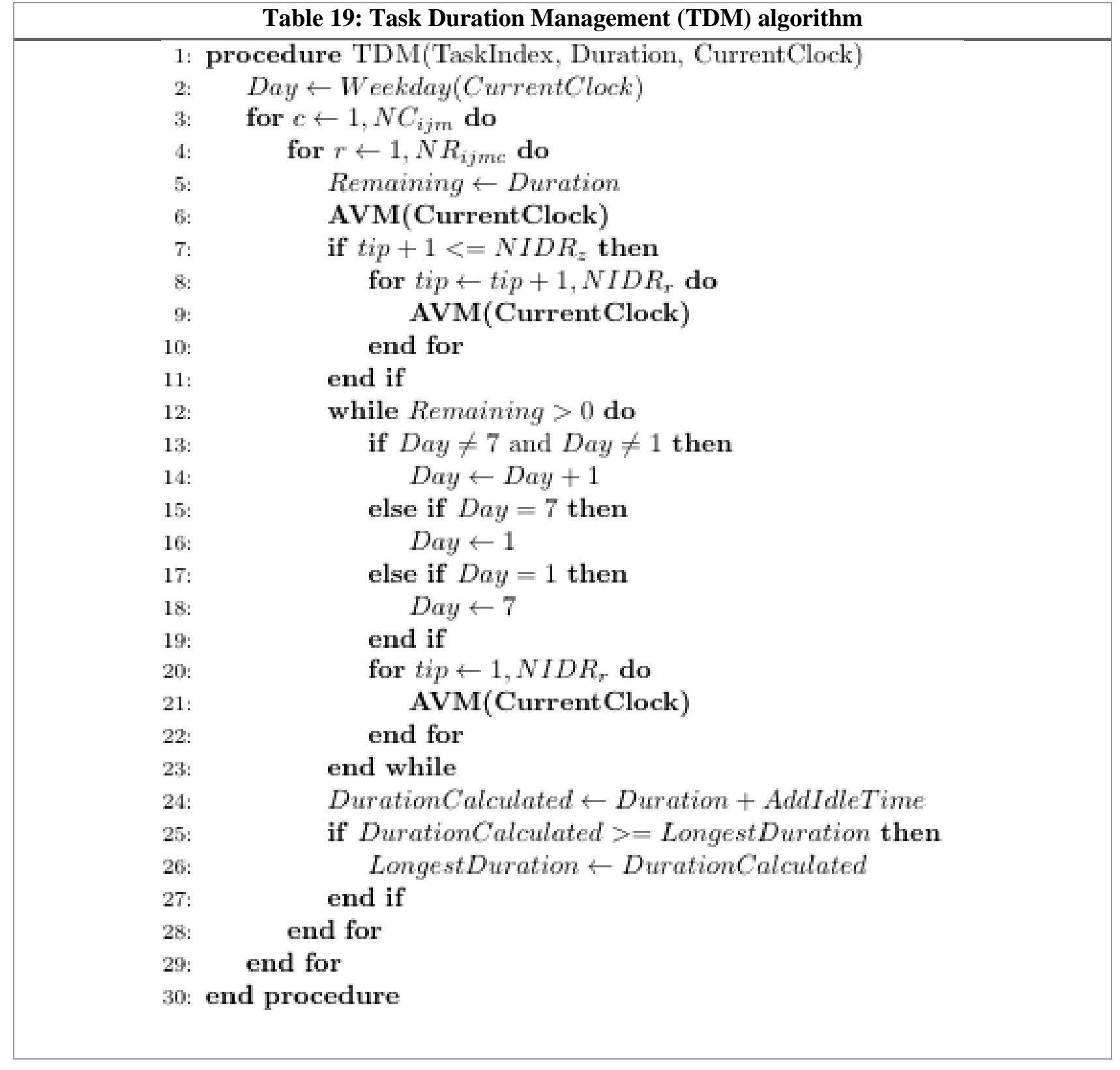




\begin{tabular}{|c|}
\hline Table 20: Availability Verification Management (AVM) algorithm \\
\hline 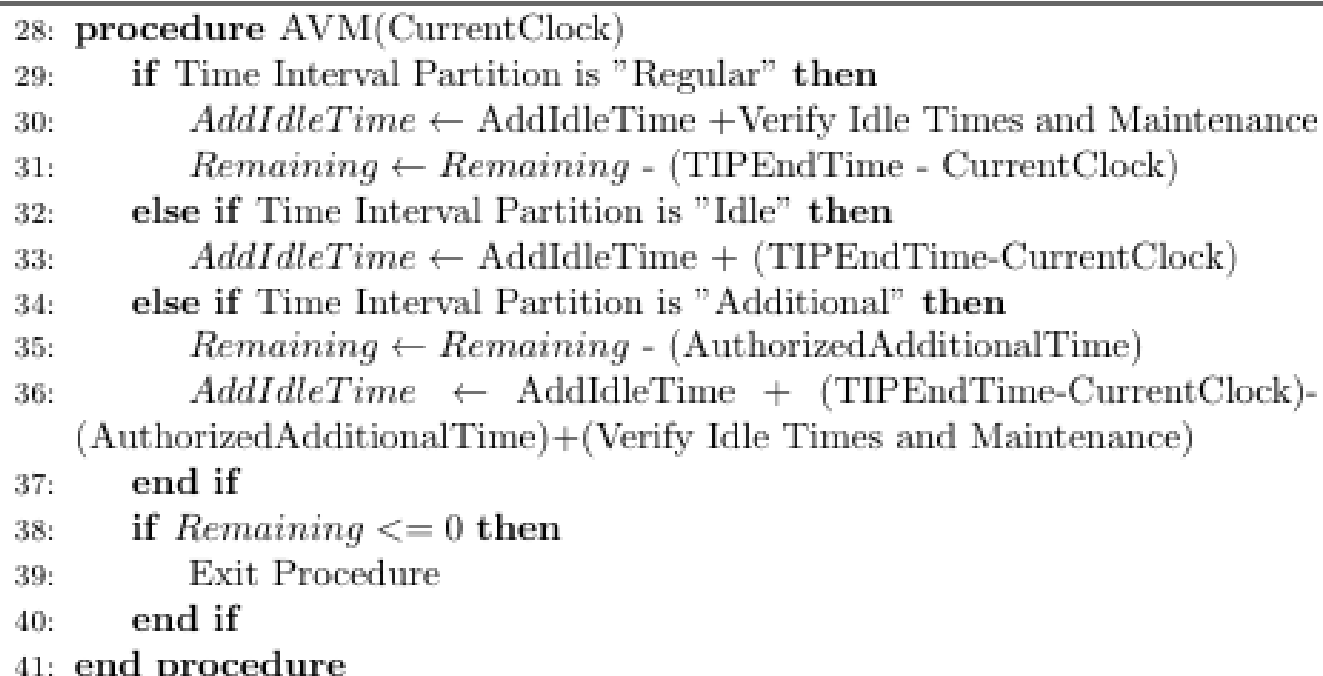 \\
\hline
\end{tabular}

Line 6 of the TDM algorithm, if the Current Clock is located within last TIP of the current day, then no more searching is required for that day. The variable TIP says which TIP the Current Clock is in. For instance, if the time of Current Clock is 1:30 PM, by looking at Table 19, the TIP uses "Regular" and variable TIP is set to 4 . If TIP is 4 , then one more TIP exists during the day. Therefore, line 6 verifies if the variable TIP +1 is less or equal to the number of TIPs during the day $\left(N I D R_{z}\right)$ for the resource with index r. If so, that means there will be more TIPs during the same day, and then $A V M$ procedure is called for each TIP (lines 7 -9). A loop is called in line 11 while Remaining is greater than 0 . Notice the variable Day is assigned to be one unit of day more at each cycle (line 12). Lines 13 to 17 are to adjust the correct value of variable Day when it is either 7 or 1 . Lines 18 to 20, once the variable Day has been assigned, all the TIPs must be run again. At each TIP, for each cycle, the $A V M$ procedure is called. When the algorithm exits loop at the line 22, the variable Duration Calculated becomes equal to original Duration plus any additional idle times preserved into the variable Add Idle Time. There is only one more check to be performed, Lines 23 to 25 guarantees that longest duration along all resources is the actual time taken to execute the task using the selected mode. Recall that one mode has several resources and each resource might have its own calendar. Therefore, the slowest resource or the most constrained one will provide the longest duration. 


\subsubsection{Task Precedence Management}

Table 21 presents the Task Precedence Management (TPM) algorithm. It shows how the simulation system handles precedence with lag-times. For tasks with no lag-time are found, DES algorithm determines how to release successors. It is triggered with a Finish Event occurrence. In the DES algorithm, release a successor may be possible when a Finish event occurs. In the TPM algorithm, on the other hand, events of "Release a Successor" are added to the list of future events, not at the current Simulation Clock.

Line 2 the variable Start is assigned to be equal to Current Clock. Line 3, the variable Finish is equal to Start plus Calculate Duration. An event of Finish Task is added into the list of events (line 4). A loop between lines 5 and 15 is performed. It runs from 1 to the number of successors for a task. For each precedence relationship having lag-time different than 0 (line 6), a Release Successor Date variable is calculated in line 7 . Lines 8 to 12 verifies if any predecessor has a finish date greater than the release date calculated, if so, Release Successor Date is assigned to be equal to Pred. Finish (predecessor finish date). Line 13 adds an event of Release Successor. $N S u c_{i j}$ is the number of successors for project $i$, task $j$; NPred $_{i j}$ is the number of predecessor for project $i$, task $j$; pre is the predecessor index,

$1 \leq$ pre $\leq N \operatorname{Pre}_{i j}$; and suc is the successor index, $1 \leq s u c \leq N S u c_{i j}$. 


\begin{tabular}{|c|c|}
\hline \multicolumn{2}{|r|}{ Table 21: Task Precedence Management algorithm (TPM) } \\
\hline \multicolumn{2}{|r|}{ 1: procedure TPM } \\
\hline & for $S u c \leftarrow 1, N S u c_{i j}$ do \\
\hline & if Lagtime $\neq 0$ then \\
\hline 4: & ReleaseSucessor Date $\leftarrow$ Finish + TaskDurationManagement ${ }_{\text {Lagtime }}$ \\
\hline 5: & N. Pred. done $\leftarrow$ N. Pred. done +1 \\
\hline & if Number of Predecessors $=\mathrm{N}$. Pred. done then \\
\hline 7: & for Pre $\leftarrow 1$, NPre $_{i j}$ do \\
\hline 8: & if Pred.Finish $>$ ReleaseSucessorDate then \\
\hline 9: & ReleaseSucessor Date $\leftarrow$ Pred.Finish \\
\hline 10: & end if \\
\hline 11: & end for \\
\hline 12: & Key $\leftarrow($ ReleaseSucessor Date, TaskIndex $)$ \\
\hline 13: & Add Event (Key, "ReleaseSuccessor", ReleaseSucessor Date, TaskIndex) \\
\hline 14: & end if \\
\hline & end if \\
\hline & end for \\
\hline 17: & d procedure \\
\hline
\end{tabular}

\subsection{System Input and Output}

The input-output schema of STREAM is shown in Figure 19. All of the outputs are based on resource allocation method of STREAM. Each resource has several associated information such as, available times, fixed cost, and variable costs. Tasks information includes, due dates, bonus, and penalty cost. Projects and tasks due dates are inputs and project and task finish dates are the computed outputs.

\begin{tabular}{|c|c|c|}
\hline \begin{tabular}{ll} 
& \multicolumn{1}{c}{ Inputs } \\
1. & Projects \\
2. & Resource Availability \\
3. & Task Precedence \\
4. & Resource \\
& Requirements \\
5. & Status \\
6. & Decisions
\end{tabular} & STREAM & \begin{tabular}{ll} 
& \multicolumn{1}{c}{ Outputs } \\
1. & Resource Utilization \\
2. & Schedule \\
3. & Procurement Issues \\
4. & Tardiness Analysis \\
5. & Cost Analysis \\
6. & Lead Times
\end{tabular} \\
\hline
\end{tabular}

Figure 19: Input-Output Schema 
Figure 20 illustrates the simulation cycle in which decisions can be made at every simulation cycle and system performance can be assessed. Outputs are discussed in Chapter 4 through the case studies.

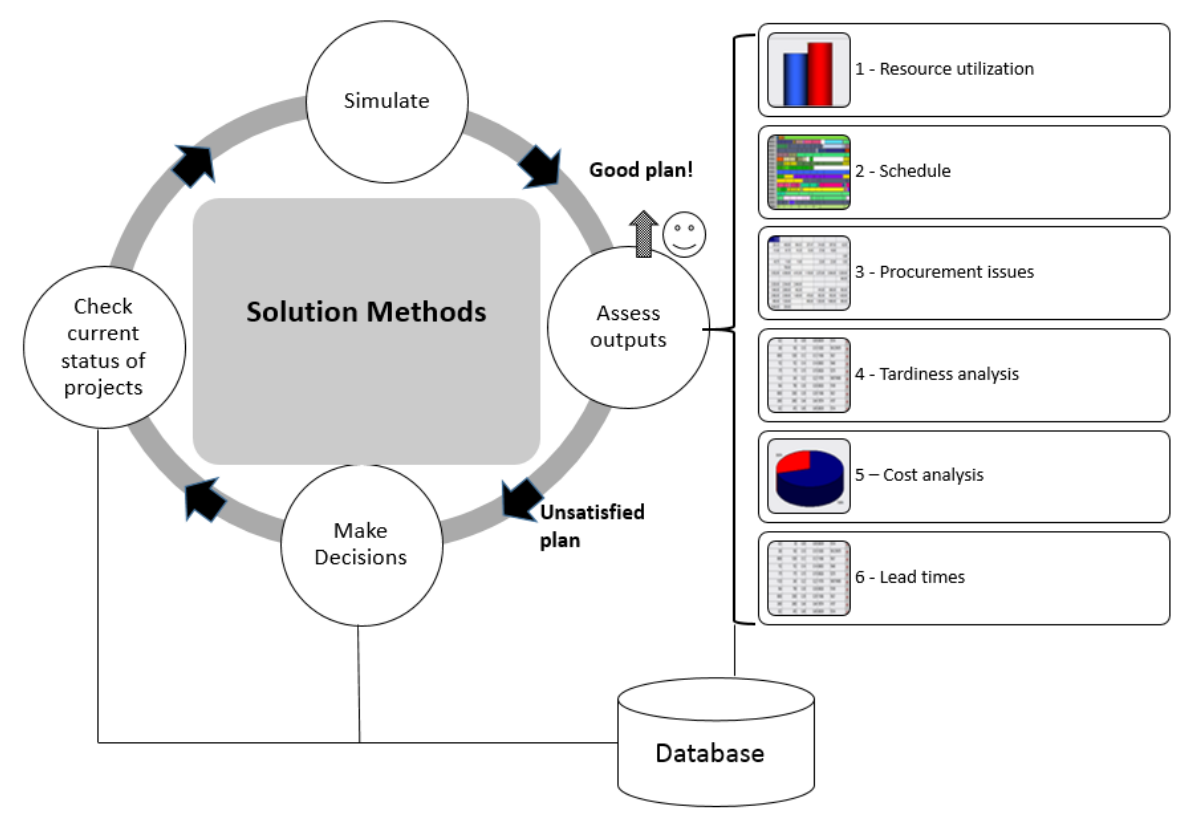

Figure 20: Simulation Cycle Schema

Two databases were implemented, one for the server side and another for the client side. The server side database maintains the simulator level data and its structure remains fixed. It has a fixed number of tables that provide all the necessary data to the simulator. By reading the data set, the proposed solution method presented in Chapter 3 is able to generate the project schedule as output.

The client side database is customized to meet the needs of particular clients or applications. Each client's database has unique data to the application. E.g., the ship repair industry requires dada that is not needed by the construction industry and vice-versa. The server side database was implemented to address all general project scheduling requirements. Customizations are carried out in the client or application database. 
The server side database utilizes 10 tables. Tables dealing with reports are located in the client side database. Structured Query Language (SQL) [SQL, 2014] was used to map data from the client database to the server database. The sever side database tables and associated fields are described in the Appendix I. Table AI.1 describes the resources. Table AI.2 describes the resource idle times. The nonrenewable resources table which carries information such as quantity on hand and on order is described in Table AI.3. Table AI.4 describes the additional times for resources. Available time for resources and multiple skilled resources are presented in Tables AI.5 and AI.6, respectively. Table AI.7 describes the tasks. Details for Task/modes, Task/Modes/Resources and task precedence are described in Tables AI.8, AI.9, and AI.10, respectively. 


\section{Chapter 4: Method Application and Validation}

Two cases studies reported in literature were carried out to test and validate STREAM. The results produce by STREAM were validated with published results. The data of the third and fourth case studies were collected on-site.

\subsection{Case study 1: Construction of a Single Cell Box Culvert}

This case study is based on the data provided by Lu et al. (2008). It deals with a construction of a single cell box culvert in a drainage project in Hong Kong. Lu et al. (2008) models the problem as optimization problem aiming to minimize the total project duration. Multi-modes, multi-skilled resources and non-renewable resources are not considered. The optimized project duration with limited resources found in Lu et al. (2008) is 275 days.

The case study is used to illustrate some of STREAM's capabilities (i.e., multi-modes, multi-skilled resources, the combination of multi-modes and multi-skilled resources, and non-renewable resources), which may impact on project's cost and duration. As the problem size is not too small and not large, these capabilities can be demonstrated through numerical examples. Additionally, as this case study provides a real-life project, it represents an invaluable context to validate STREAM. In this research, the work of Lu et al. (2008) is strictly used for obtaining data from their case study. A comparison between the proposed approach and Lu et al. (2008)'s approach is not made as the goals and capabilities of both approaches are by nature different.

This case study is organized in four subsections; 1) Input data, 2) The set of scenarios used to validate STREAM are described, 3) Results applying STREAM, and 4) Compares the results of the assessed scenarios. 


\subsubsection{Inputs}

Construction of a single cell box culvert project involves 8 different types of resources and 33 tasks. The project assumed eight hours per work day and distinct calendars were used for different resources. Table 22 shows the tasks, their durations, and their predecessors. The resources are shown in Table 23. The resources are: Bar bender and fixer (BBF), Backhoe with excavator (BE), Crawler mounted crane (CMC), Carpenter (CF), Concreting Labour (CLB), Drain Layer (DL), Skilled labour (LB) and Roller (RR). Figure 21 shows the Task screen and Figure 22 shows the Resource screen in STREAM. The resource calendars are displayed in Table 24. Appendix II describes the data files associated with this case study. Table AII.1 shows task resource requirements for mode 1 and resource capacity limits. The original case study assumes only one mode for each task. To demonstrate STREAM's capabilities, information regarding multi-modes, multi-skilled workers, non-renewable resources and costs are used as additional inputs in this case study.

Regarding multi-mode tasks (as tasks may be performed by selecting one out of several combinations of resource requirements), an additional mode (mode 2) was included for each task. For instance, according to mode 1, task A lasts 16 days and requires four BEs and eight LBs, and according to mode 2, it lasts 23 days and requires two BEs and five LBs. Table AII.2 in the Appendix II shows task resource requirements for mode 2. Resource skills are categorized as excellent, good, and satisfactory (Table 25). As example pf skills levels of each resource is shown in Table 26. E.g., resources CF perform CF tasks with excellence, CLB tasks in a good manner and LB tasks in a satisfactory way. Resources CLB perform CLB tasks with excellence, CF tasks in a good manner and LB tasks in a satisfactory way. To evaluate the financial impact of a scenario, STREAM requires data for fixed costs, variable costs, penalty costs, and bonus costs. Additionally, due dates for tasks are also relevant as they represent major milestones commonly found in project management. An example of fixed and variable costs for the resources is shown in Table AII.3 in the Appendix II. STREAM allows for penalty cost and bonus. Bonus is for early task completion and penalty is for delayed tasks. Bonus and penalty are calculated by comparing task 
finish time and task due date. These costs can be ignored by providing zero values for penalty costs and bonus. Penalty cost and bonus are in terms of loss and profit per day, respectively. Table AII.4 in the Appendix II shows task costs. A cost/time trade-off analysis is relevant and can be assessed when multiskilled workers exist (if a task requires a CLB skill and a LB is assigned to the task, the cost associated refers to the LB worker).

\begin{tabular}{|c|c|c|c|c|}
\hline \multicolumn{5}{|c|}{ Table 22: Task Description, Duration, and Predecessors } \\
\hline & Task & Description & Duration & Pred. \\
\hline 1 & A & General excavation & 16 & \\
\hline 2 & B1 & Excavation of additional $2 \mathrm{~m}$ thick marine mud & 8 & A \\
\hline 3 & B2 & Deposition and compaction of $2 \mathrm{~m}$ thick additional rock fill materials & 16 & B1 \\
\hline 4 & B3 & Placing and compaction of $400 \mathrm{~mm}$ thick rock fill & 12 & B2 \\
\hline 5 & B4 & Laying of $75 \mathrm{~mm}$ thick blinding concrete & 3 & B3 \\
\hline 6 & B5 & Fixing of steel reinforcement for base slab \& side walls (lower part) & 32 & B4 \\
\hline 7 & B6 & Erection of formwork for base slab \& side walls (lower part) & 16 & B4 \\
\hline 8 & B7 & Concreting of base slab \& side walls (lower part) & 4 & B5,B6 \\
\hline 9 & B8 & Erection of false work for top slab & 32 & B7 \\
\hline 10 & B9 & Fixing of steel reinforcement for top slab \& side walls (upper part) & 20 & B8 \\
\hline 11 & B10 & Erection of formwork for top slab \& wide walls (upper part) & 20 & B8 \\
\hline 12 & B11 & Concreting of top slab \& side walls (upper part) & 5 & B9,B10 \\
\hline 13 & $\mathrm{C} 1$ & Placing and compaction of $400 \mathrm{~mm}$ thick rock fill & 12 & A \\
\hline 14 & $\mathrm{C} 2$ & Laying of $75 \mathrm{~mm}$ thick blinding concrete & 3 & $\mathrm{C} 1$ \\
\hline 15 & $\mathrm{C} 3$ & Fixing of steel reinforcement for base slab \& side walls (lower part) & 32 & $\mathrm{C} 2$ \\
\hline 16 & $\mathrm{C} 4$ & Erection of formwork for base slab \& side walls (lower part) & 16 & $\mathrm{C} 2$ \\
\hline 17 & C5 & Concreting of base slab \& side walls (lower part) & 3 & $\mathrm{C} 3, \mathrm{C} 4$ \\
\hline 18 & C6 & Erection of false work for top slab & 32 & C5 \\
\hline 19 & $\mathrm{C} 7$ & Fixing of steel reinforcement for top slab \& side walls (upper part) & 20 & C6 \\
\hline 20 & $\mathrm{C} 8$ & Erection of formwork for top slab \& wide walls (upper part) & 20 & C6 \\
\hline 21 & C9 & Concreting of top slab \& side walls (upper part) & 5 & $\mathrm{C} 7, \mathrm{C} 8$ \\
\hline 22 & D1 & Placing and compaction of $400 \mathrm{~mm}$ thick rock fill & 2 & C9 \\
\hline 23 & D2 & Laying of $75 \mathrm{~mm}$ thick blinding concrete & 1 & D1 \\
\hline 24 & D3 & Fixing of steel reinforcement for base slab \& side walls (lower part) & 5 & D2 \\
\hline 25 & D4 & Erection of formwork for base slab \& side walls (lower part) & 2 & D3 \\
\hline 26 & D5 & Concreting of base slab \& side walls (lower part) & 1 & D4 \\
\hline 27 & D6 & Erection of false work for top slab & 4 & D5,E2 \\
\hline 28 & D7 & Fixing of steel reinforcement for top slab \& side walls (upper part) & 6 & D6 \\
\hline 29 & D8 & Erection of formwork for top slab \& wide walls (upper part) & 3 & D7 \\
\hline 30 & D9 & Concreting of top slab \& side walls (upper part) & 1 & D6,D7 \\
\hline 31 & E1 & Approval on concrete pipe manufacturer \& delivery of concrete pipe & 90 & \\
\hline 31 & E2 & Positioning of pre-cast concrete pipes at end wall & 1 & E1 \\
\hline 33 & $\mathrm{~F}$ & Backfilling \& compaction B11, D9 7484 Available resources limit & 7 & B11,D9 \\
\hline
\end{tabular}


Tasks

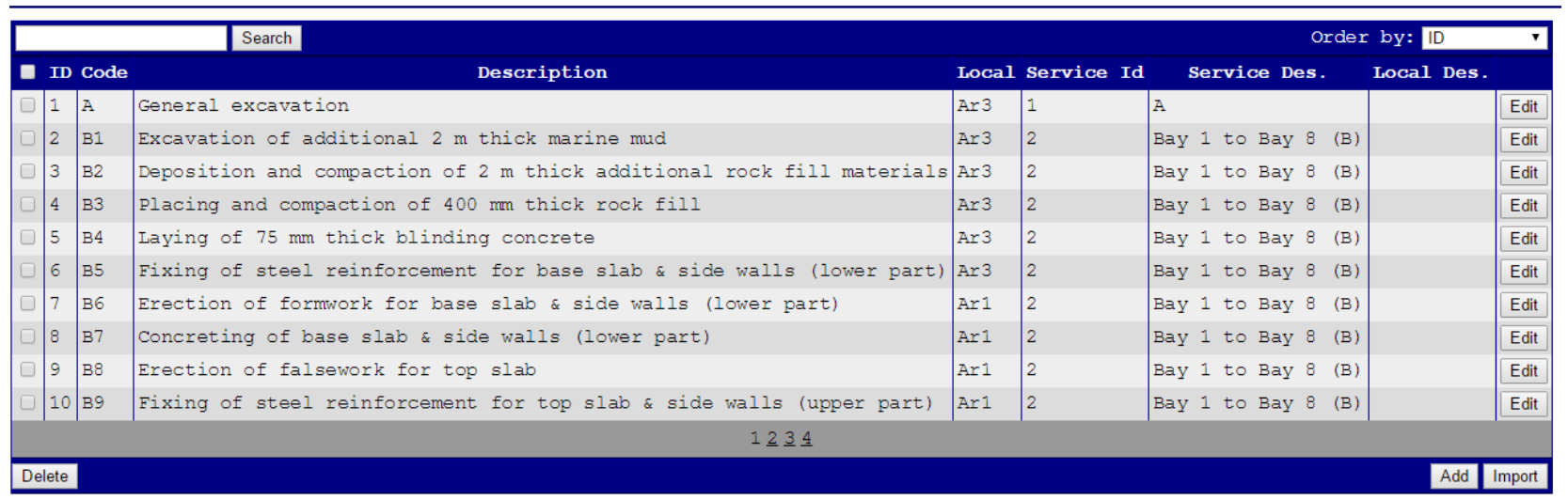

Figure 21: Task screen in STREAM

Table 23: Resource Description

\begin{tabular}{|l|l|c|}
\hline \multicolumn{1}{|c|}{ Resource } & \multicolumn{1}{|c|}{ Description } & Limits \\
\hline BBF & Bar bender and fixer & 4 \\
\hline BE & Backhoe with excavator & 4 \\
\hline CMC & Crawler mounted crane & 3 \\
\hline CF & Carpenter & 2 \\
\hline CLB & Concreting labour & 5 \\
\hline DL & Drain layer & 1 \\
\hline LB & Skilled labour & 8 \\
\hline RR & Roller & 4 \\
\hline
\end{tabular}

Resources

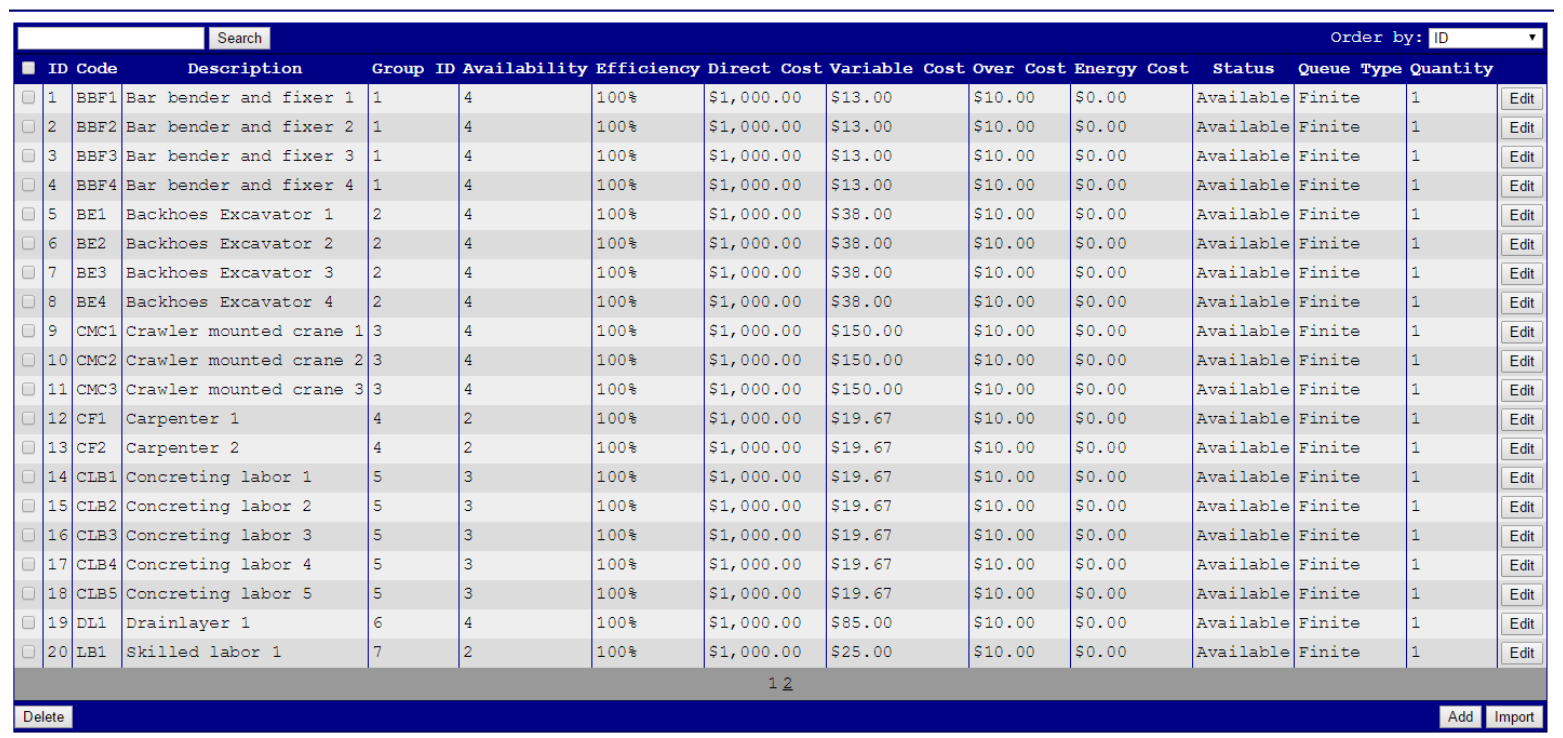

Figure 22: Resource screen in STREAM 
Table 24: Resource Calendar

\begin{tabular}{|l|c|l|}
\hline Resource & Number of days/week & \\
\hline BBF & 7 & M, W, T, Th., F, S, Su. \\
\hline BE & 7 & M, W, T, Th., F, S, Su. \\
\hline CMC & 7 & M, W, T, Th., F, S, Su. \\
\hline CF & 6 & M, W, T, Th., F, S \\
\hline CLB & 4 & M, W, T, Th. \\
\hline DL & 7 & M, W, T, Th., F, S, Su. \\
\hline LB & 6 & M, W, T, Th., F, S \\
\hline RR & 7 & M, W, T, Th., F, S, Su. \\
\hline
\end{tabular}

\begin{tabular}{|c|l|}
\hline \multicolumn{2}{|c|}{ Table 25: Resource Skill Levels } \\
\hline Level & \multicolumn{1}{|c|}{ Description } \\
\hline 0 & None \\
\hline 1 & Excellent \\
\hline 2 & Good \\
\hline 3 & Satisfactory \\
\hline
\end{tabular}

Table 26: Multiple Skill Example

\begin{tabular}{|l|c|c|c|c|c|c|c|}
\hline \multicolumn{7}{|c|}{ Skill level } \\
\hline \multicolumn{1}{|c|}{ Resource } & BBF & CMC & CF & CLB & DL & LB & RR \\
\hline BBF & 1 & 0 & 0 & 0 & 0 & 0 & 0 \\
\hline CMC & 0 & 1 & 0 & 0 & 0 & 0 & 0 \\
\hline CF & 0 & 0 & 1 & 2 & 0 & 3 & 0 \\
\hline CLB & 0 & 0 & 2 & 1 & 0 & 3 & 0 \\
\hline DL & 0 & 0 & 0 & 0 & 1 & 0 & 0 \\
\hline LB & 0 & 0 & 3 & 2 & 0 & 1 & 0 \\
\hline RR & 0 & 0 & 0 & 0 & 0 & 0 & 1 \\
\hline
\end{tabular}




\subsubsection{Scenarios}

Seven different scenarios were considered for this case study. Scenarios 1, 2, and 3 are the same as those considered by Lu et al. (2008). Scenarios 4, 5, 6, and 7 utilize multi-skills, multi-modes, multi-skills and modes, and non-renewable resources respectively. Brief description of each scenario is as follows:

Scenario 1: Determine schedule based on task precedence, resource calendars and resource availability.

Scenario 2: $\quad$ Minimize Total Project Duration (TPD), while maintaining task precedence, resource calendars, and resource availability.

Scenario 3: Minimize TPD by finding the optimum quantity of resources, while maintaining task precedence, and resource calendars.

Scenario 4: Determine schedule based on task precedence, resource calendars and resource availability constraints, where human resources can have multiple skills.

Scenario 5: Determine schedule based on task precedence, resource calendars and resource availability constraints, where tasks can be carried out in multiple ways (multi-modes).

Scenario 6: Determine schedule based on task precedence, resource calendars and resource availability constraints, where human resources can have multiple skills and tasks can be carried out in multiple ways (multi-modes).

Scenario 7: Determine schedule based on task precedence, resource calendars and resource availability constraints, where non-renewable resources and replenishment plan are considered. 


\subsubsection{Results applying STREAM}

The seven scenarios were tested using STREAM. Each one reveals a particular capability of the proposed solution approach in providing resource allocation flexibilities.

\section{Case study 1: Scenarios 1 and 2}

For scenarios 1 and 2 STREAM found the same results. STREAM found TPD to be 275 days as in Lu et al. (2008) and cost of $\$ 14,347,972$. STREAM provided addition insight to the project. Skilled labour (LB) had the highest utilization (93.35\%), whereas the drain layers (DL) had the lowest utilization $(0.14 \%)$. The project manager may consider that LB as a cause of resource bottleneck and he/she may choose to add another LB. STREAM does not permit over allocation of resources and delays are automatically computed. The resource utilization computed and the required quantity for each type of resource is described in Table 27. The detailed schedule is shown in Table 28. Figure 23 shows the Resource Utilization screen in STREAM. Each bar shows the idle and usage percentage time for each resource assigned for tasks. Red presents idle time percentage whereas blue is use percentage time.

\section{Table 27: Total Project Duration and Resource Utilization by STREAM for Scenario 1 and 2}

\begin{tabular}{|l|c|c|}
\hline \multicolumn{1}{|c|}{ Resource } & STREAM & Resource utilization (\%) \\
\hline BBF & 4 & 43.29 \\
\hline BE & 4 & 19.68 \\
\hline CMC & 3 & 34.53 \\
\hline CF & 2 & 31.51 \\
\hline CLB & 5 & 11.83 \\
\hline DL & 1 & 0.14 \\
\hline LB & 8 & $\mathbf{9 3 . 3 5}$ \\
\hline RR & 4 & 13.38 \\
\hline Total Project Duration (TPD) & 275 & \\
\hline
\end{tabular}




\begin{tabular}{|c|c|c|c|c|}
\hline \multicolumn{5}{|c|}{ Table 28: Detailed Schedule by STREAM for Scenario 1 and 2} \\
\hline Task & Start Date & End Date & Due Date & Deviation (d) \\
\hline A & $5 / 28 / 2015$ & $6 / 15 / 2015$ & $6 / 16 / 2015$ & -0.29 \\
\hline B1 & $6 / 16 / 2015$ & $6 / 24 / 2015$ & $6 / 26 / 2015$ & -1.29 \\
\hline B2 & $6 / 25 / 2015$ & $7 / 13 / 2015$ & $7 / 12 / 2015$ & 1.71 \\
\hline B3 & $8 / 1 / 2015$ & $8 / 14 / 2015$ & $7 / 28 / 2015$ & 17.71 \\
\hline B4 & $9 / 22 / 2015$ & $9 / 24 / 2015$ & $8 / 1 / 2015$ & 54.71 \\
\hline B5 & $10 / 20 / 2015$ & $11 / 25 / 2015$ & $9 / 21 / 2015$ & 65.71 \\
\hline B6 & $10 / 20 / 2015$ & $11 / 6 / 2015$ & $8 / 15 / 2015$ & 83.71 \\
\hline B7 & $11 / 26 / 2015$ & $12 / 2 / 2015$ & $9 / 14 / 2015$ & 79.71 \\
\hline B8 & $12 / 3 / 2015$ & $1 / 8 / 2016$ & $10 / 21 / 2015$ & 79.71 \\
\hline B9 & $1 / 14 / 2016$ & $2 / 5 / 2016$ & $11 / 20 / 2015$ & 77.71 \\
\hline B10 & $1 / 9 / 2016$ & $2 / 1 / 2016$ & $11 / 20 / 2015$ & 73.71 \\
\hline B11 & $2 / 9 / 2016$ & $2 / 16 / 2016$ & $11 / 26 / 2015$ & 82.71 \\
\hline $\mathrm{C} 1$ & $6 / 16 / 2015$ & $6 / 29 / 2015$ & $6 / 30 / 2015$ & -0.29 \\
\hline $\mathrm{C} 2$ & $6 / 30 / 2015$ & $7 / 2 / 2015$ & $7 / 2 / 2015$ & 0.71 \\
\hline C3 & $7 / 3 / 2015$ & $8 / 8 / 2015$ & $8 / 15 / 2015$ & -6.29 \\
\hline $\mathrm{C} 4$ & $7 / 14 / 2015$ & $7 / 31 / 2015$ & $8 / 15 / 2015$ & -14.29 \\
\hline $\mathrm{C} 5$ & $8 / 10 / 2015$ & $8 / 13 / 2015$ & $8 / 18 / 2015$ & -4.29 \\
\hline C6 & $8 / 15 / 2015$ & $9 / 21 / 2015$ & $9 / 20 / 2015$ & 1.71 \\
\hline $\mathrm{C} 7$ & $9 / 26 / 2015$ & $10 / 19 / 2015$ & $10 / 21 / 2015$ & -1.29 \\
\hline $\mathrm{C} 8$ & $9 / 26 / 2015$ & $10 / 19 / 2015$ & $10 / 21 / 2015$ & -1.29 \\
\hline C9 & $11 / 9 / 2015$ & $11 / 16 / 2015$ & $10 / 25 / 2015$ & 22.71 \\
\hline D1 & $11 / 17 / 2015$ & $11 / 18 / 2015$ & $10 / 28 / 2015$ & 21.71 \\
\hline D2 & $11 / 19 / 2015$ & $11 / 19 / 2015$ & $12 / 2 / 2015$ & -12.29 \\
\hline D3 & $11 / 20 / 2015$ & $11 / 25 / 2015$ & $12 / 5 / 2015$ & -9.29 \\
\hline D4 & $11 / 26 / 2015$ & $11 / 27 / 2015$ & $12 / 8 / 2015$ & -10.29 \\
\hline D5 & $12 / 3 / 2015$ & $12 / 3 / 2015$ & $1 / 10 / 2016$ & -37.29 \\
\hline D6 & $1 / 9 / 2016$ & $1 / 13 / 2016$ & $1 / 10 / 2016$ & 3.71 \\
\hline D7 & $2 / 2 / 2016$ & $2 / 8 / 2016$ & $1 / 13 / 2016$ & 26.71 \\
\hline D8 & $2 / 6 / 2016$ & $2 / 9 / 2016$ & $1 / 13 / 2016$ & 27.71 \\
\hline D9 & $2 / 17 / 2016$ & $2 / 17 / 2016$ & $1 / 25 / 2016$ & 23.71 \\
\hline E1 & $5 / 28 / 2015$ & $8 / 25 / 2015$ & $8 / 30 / 2015$ & -4.29 \\
\hline E2 & $9 / 25 / 2015$ & $9 / 25 / 2015$ & $9 / 2 / 2015$ & 23.71 \\
\hline $\mathrm{F}$ & $2 / 18 / 2016$ & $2 / 25 / 2016$ & $2 / 20 / 2016$ & 5.71 \\
\hline
\end{tabular}




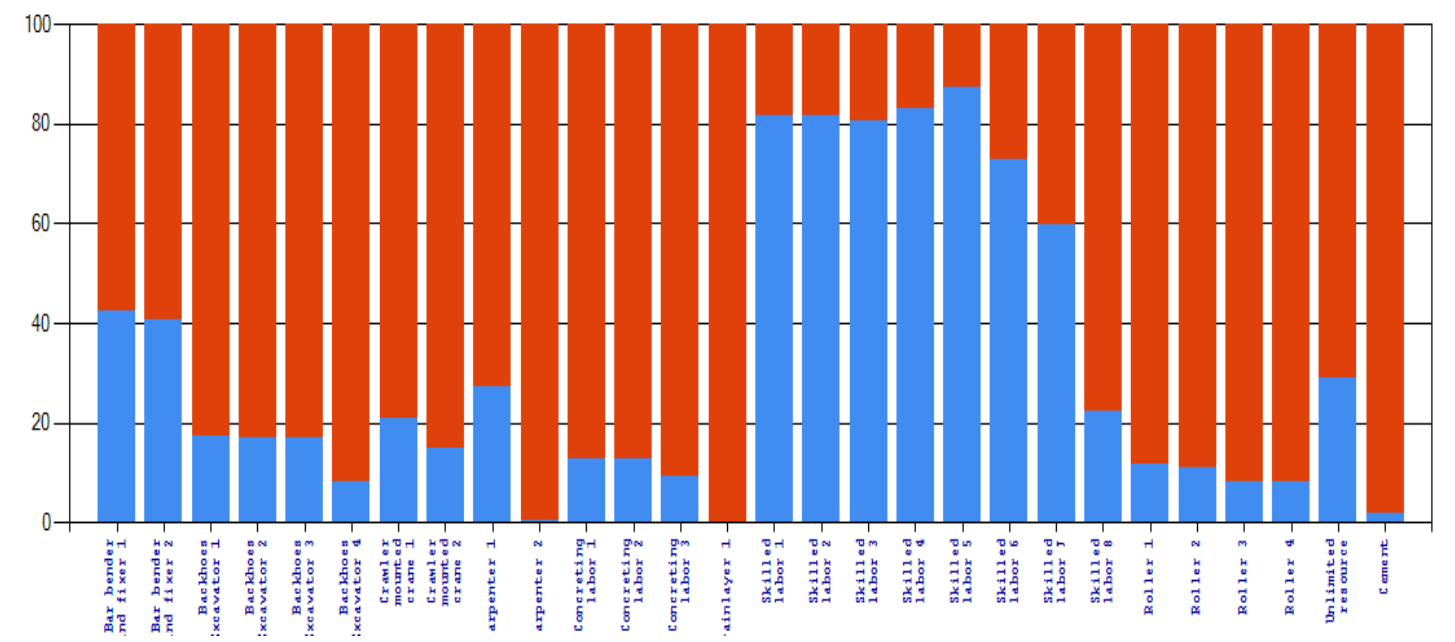

Figure 23: Resource Utilization Screen in STREAM

\section{Case study 1 scenario 3: Finding the optimal quantity of resources}

In this scenario 3 , the objective is to find the optimal number of resource units for the aforementioned bottleneck resource LB. STREAM utilizes a simulation approach to determine the optimal number. Starting from the original 8 units of LB, one additional unit was considered at each simulation run. Table 29 shows ten simulation runs. After the eighth run STREAM does not find any improvement in total project duration and cost as shown in Table 29. Resource utilization with additional LBs for scenario 3 is shown in Table 30. The detailed schedule for scenario 3 is presented in Table AII.5 in the Appendix II. Figures 24 and 25 show project duration and cost as a function of skilled labors, respectively. Figure 26 shows a linear increase in project cost when more than fifteen skilled labor is used.

Table 29: Cost Analysis by STREAM for Scenario 3

\begin{tabular}{|c|c|c|c|c|c|c|c|}
\hline & LB & Project & Start date & Finish date & \% Time & Total Project & \% Cost \\
\hline $\mathbf{1}$ & $\mathbf{8}$ & $\mathbf{2 7 5}$ & $\mathbf{5 / 2 8 / 2 0 1 5}$ & $\mathbf{2 / 2 5 / 2 0 1 6}$ & & $\mathbf{\$ 1 4 , 3 4 7 , 9 7 2}$ & \\
\hline 2 & 9 & 268 & $5 / 28 / 2015$ & $2 / 18 / 2016$ & $2.55 \%$ & $\$ 13,344,318$ & $6.83 \%$ \\
\hline 3 & 10 & 261 & $5 / 28 / 2015$ & $2 / 11 / 2016$ & $5.09 \%$ & $\$ 11,623,640$ & $18.99 \%$ \\
\hline 4 & 11 & 225 & $5 / 28 / 2015$ & $1 / 6 / 2016$ & $18.18 \%$ & $\$ 6,256,752$ & $56.39 \%$ \\
\hline 5 & 12 & 218 & $5 / 28 / 2015$ & $12 / 30 / 2015$ & $20.73 \%$ & $\$ 5,187,256$ & $63.85 \%$ \\
\hline 6 & 13 & 190 & $5 / 28 / 2015$ & $12 / 2 / 2015$ & $30.91 \%$ & $\$ 2,253,504$ & $84.29 \%$ \\
\hline 7 & 14 & 190 & $5 / 28 / 2015$ & $12 / 2 / 2015$ & $30.91 \%$ & $\$ 2,254,504$ & $84.29 \%$ \\
\hline $\mathbf{8}$ & $\mathbf{1 5}$ & $\mathbf{1 8 5}$ & $\mathbf{5 / 2 8 / 2 0 1 5}$ & $\mathbf{1 1 / 2 7 / 2 0 1 5}$ & $\mathbf{3 2 . 7 3 \%}$ & $\mathbf{\$ 1 , 8 9 8 , 0 0 7}$ & $\mathbf{8 6 . 7 7 \%}$ \\
\hline 9 & 16 & 185 & $5 / 28 / 2015$ & $11 / 27 / 2015$ & $32.73 \%$ & $\$ 1,899,007$ & $86.76 \%$ \\
\hline 10 & 17 & 185 & $5 / 28 / 2015$ & $11 / 27 / 2015$ & $32.73 \%$ & $\$ 1,900,007$ & $86.76 \%$ \\
\hline
\end{tabular}


Table 30: Total Resource Duration and Resource Utilization by STREAM for Scenario 3

\begin{tabular}{|l|c|c|}
\hline \multicolumn{1}{|c|}{ Resource } & STREAM & Resource utilization (\%) \\
\hline BBF & 4 & 34.31 \\
\hline BE & 4 & 25.13 \\
\hline CMC & 3 & 26.47 \\
\hline CF & 2 & 23.20 \\
\hline CLB & 5 & 13.97 \\
\hline DL & 1 & 0.20 \\
\hline LB & 15 & 61.16 \\
\hline RR & 4 & 16.64 \\
\hline Total Project Duration (TPD) & 185 & \\
\hline
\end{tabular}

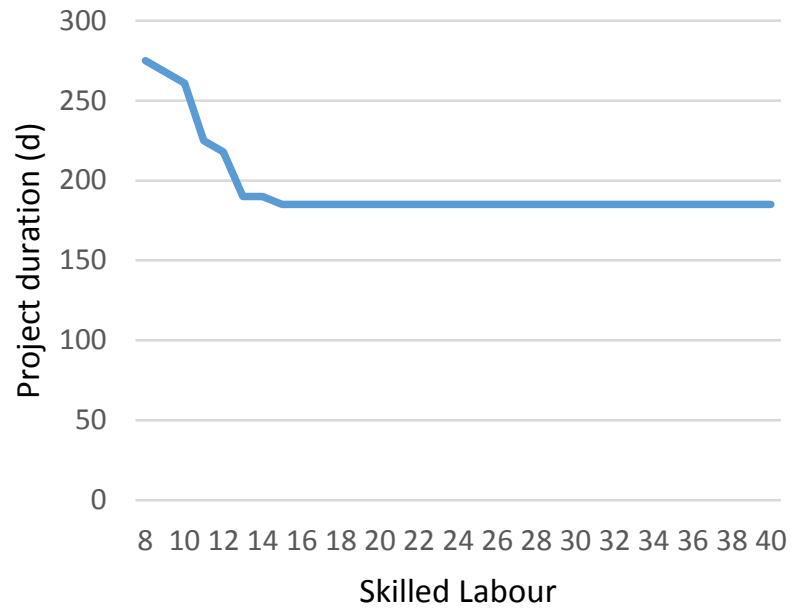

Figure 24: Project Duration (days)

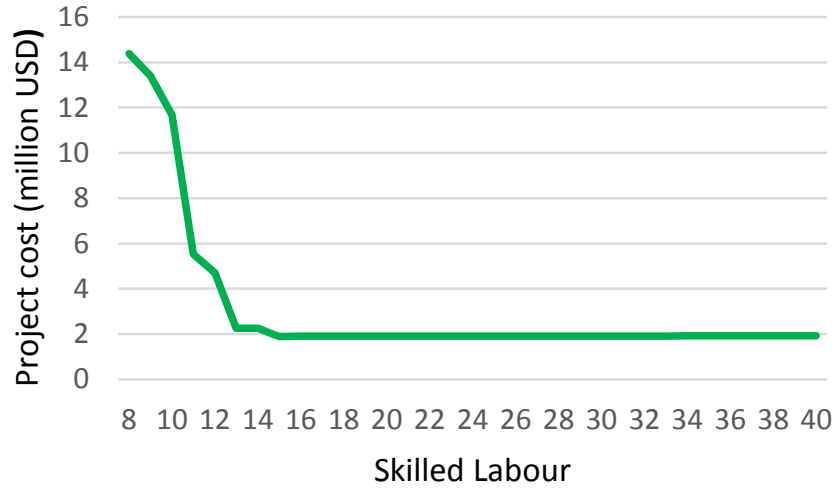

Figure 25: Project Cost (million USD) 


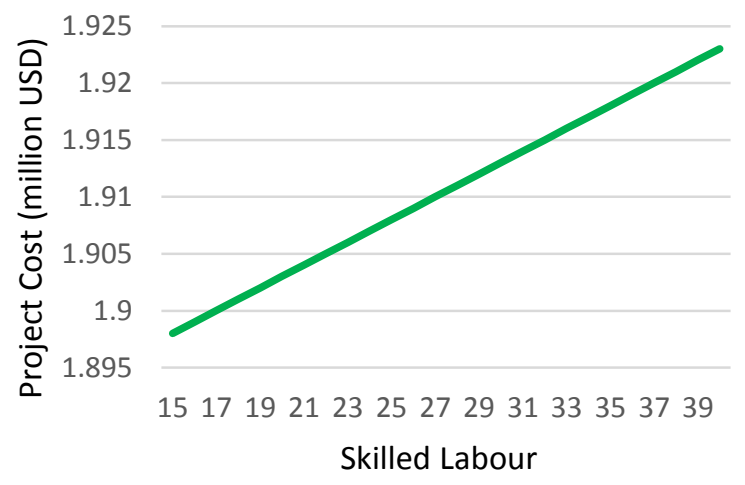

Figure 26: Project Cost (million USD) for Skilled Labour between 15 and 38

\section{Case study 1: Scenario 4: Human resources with multiple skills}

This scenario deals with the case of human resources having multi-skills. The assumptions are that: resource calendars, task precedence and task durations are the same as in scenario 1, the quantity of resources used is either equal to or less than scenario 1, but labours have multiple skills.

Four different simulation runs were carried out for this scenario. In run 1, the LBs have CF and CLB skills, and all CFs and CLBs were removed. The limit on LBs was kept the same as in Table 23. In run 2, the CLBs have CF skills, and all CFs were removed. The number of CLBs was kept the same as in Table 23. In run 3, LBs have additional CF skills and the two CFs were removed. The number of LBs was kept the same as in Table 23. In run 4, LBs, CFs and CLBs have three skills each. The number of LBs, CFs and CLBs was kept the same as in Table 23.

Table 31 shows the total project duration and total project cost for the four simulation runs. The total project duration and total project cost for the four simulation runs are compared with the baseline scenario with TPD of 275 days. The negative value of duration (days) refers to additional days required, compared to the baseline value. Negative values on cost difference refer to the increase in cost considering the baseline value. Figures 27 and 28 show project durations and costs based on the four simulation runs 


\begin{tabular}{|c|c|c|c|c|c|c|c|}
\hline \multicolumn{9}{|c|}{ Table 31: Total Project Duration and Cost Analysis by STREAM for Scenario 4 } \\
\hline & TBD & Start date & Finish date & $\%$ Time & Total Project & Cost & \% Cost \\
\hline 1 & 370 & $5 / 28 / 2015$ & $5 / 30 / 2016$ & $-34.55 \%$ & $\$ 39,801,807$ & $-\$ 25,453,834$ & $-177.40 \%$ \\
\hline 2 & 304 & $5 / 28 / 2015$ & $3 / 25 / 2016$ & $-10.55 \%$ & $\$ 22,289,949$ & $-\$ 7,941,976$ & $-55.35 \%$ \\
\hline 3 & 296 & $5 / 28 / 2015$ & $3 / 18 / 2016$ & $-7.64 \%$ & $\$ 17,143,265$ & $-\$ 2,795,292$ & $-19.48 \%$ \\
\hline 4 & 224 & $5 / 28 / 2015$ & $1 / 5 / 2016$ & $18.55 \%$ & $\$ 7,923,455$ & $\$ 6,424,517$ & $44.78 \%$ \\
\hline
\end{tabular}

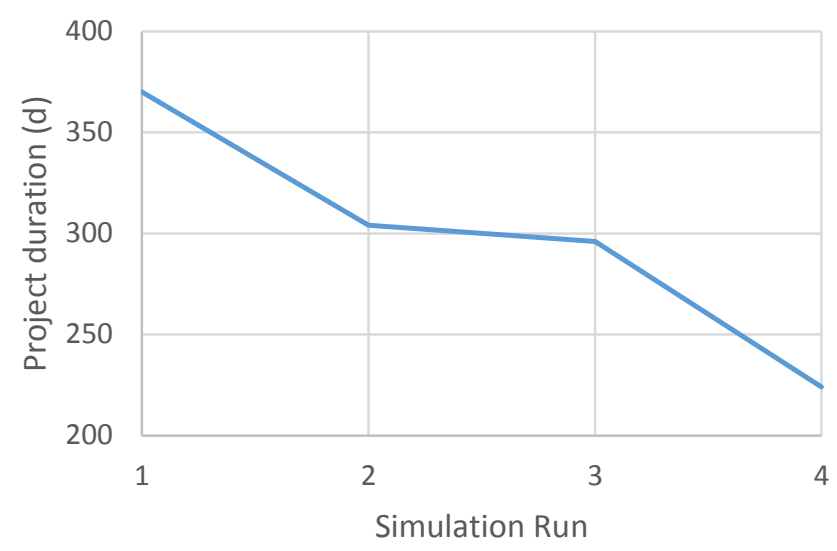

Figure 27: Project Duration (days) for Four Simulation Runs

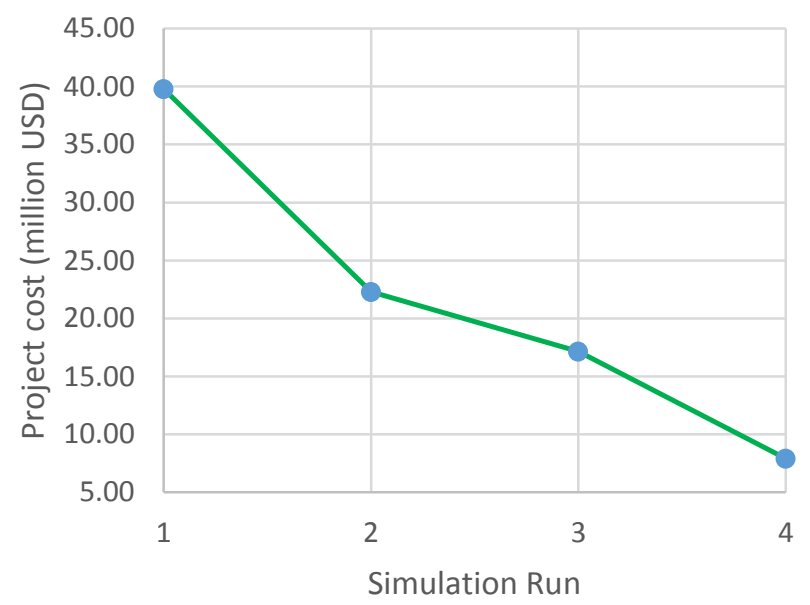

Figure 28: Project Cost (million USD) for Four Simulation Runs

Simulation run number four of scenario 4 provides the "best" solution, where TPD is reduced from 275 days to 224 days (51 fewer days), and total project cost is reduced from $\$ 14,347,972$ to $\$ 7,923,455$, a $44.78 \%$ cost reduction amounting to $\$ 6,424,517$. 


\section{Case study 1: Scenario 5: Tasks with multiple modes}

This scenario deals with the case of multi-modes. The assumptions for this scenario are similar to scenario 4, except for that labour does not have multiple skills, but multi-modes are allowed now. Figure 29 shows the Task/Modes screen of STREAM. It shows two mode for Task 2.

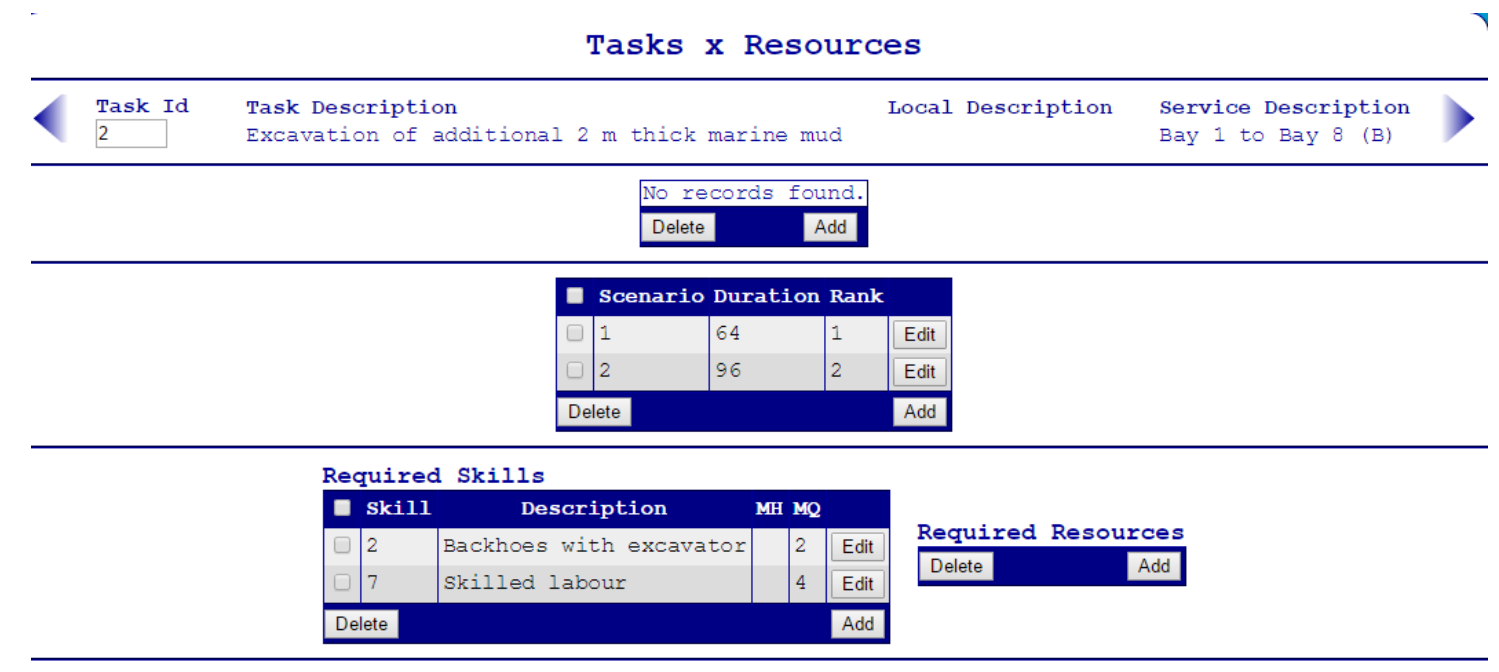

Figure 29: Task/Mode/Resources screen in STREAM

Under this scenario, TPD is reduced from 275 days to 255 days (20 fewer days) and total project cost is reduced from $\$ 14,347,972$ to $\$ 10,855,420$, a $24.3 \%$ cost reduction amounting to $\$ 3,492,551$, as shown in Table 32.

\section{Table 32: Project Duration and Cost Analysis by STREAM for Scenario 5}

\begin{tabular}{|c|c|c|c|c|c|c|}
\hline TPD & Start date & Finish & Total Project & Total Cost for 275 & Dif. in Cost & Dif. in days \\
\hline 255 & $5 / 28 / 2015$ & $2 / 5 / 2016$ & $\$ 10,855,420$ & $\$ 14,347,972$ & $\$ 3,492,551$ & 20 \\
\hline
\end{tabular}


Case study 1: Scenario 6: Scheduling considering that humans have multiple skills and that tasks have multiple modes

This scenario deals with the case of multi-skills and multi-modes. The assumptions for this scenario are the same as in scenario 4, but project tasks can also have multi-modes. Under this scenario, project duration is reduced from 275 days to 218 days (57 fewer days) and total project cost is reduced from $\$ 14,347,972$ to $\$ 6,460,206$, a cost reduction of nearly $50 \%$, amounting to $\$ 7,887,765$, as shown in Table 33. Table AII.6 in the Appendix II shows the detailed schedule, along with schedule deviations, as generated by STREAM for this scenario.

Table 33: Project Duration and Cost Analysis by STREAM for Scenario 6

\begin{tabular}{|c|c|c|c|c|c|c|}
\hline TPD & Start date & Finish date & Total Project & Total Cost for 275 & Dif. in & Dif. in days \\
\hline 218 & $5 / 28 / 2015$ & $12 / 30 / 2015$ & $\$ 6,460,206$ & $\$ 14,347,972$ & $\$ 7,887,765$ & 57 \\
\hline
\end{tabular}

\section{Case study 1: Scenario 7: Resources can be non-renewable or renewable}

This scenario deals with non-renewable resources with replenishment plan (doubly constrained resource). This scenario is proposed to demonstrate how STREAM can warn project managers on nonrenewable resources shortages with a replenishment plan. It does not consider multi-modes and multiskills capabilities for simplicity purposes, although all the presented capabilities can be used simultaneously. In other words, the assumptions for this scenario are the same as in scenario 1, but a nonrenewable resource is also required for a given task.

In this scenario, task $\mathrm{C} 2$, in addition to its original resource requirements shown in Table AII.1 described in the Appendix II, also requires $100 \mathrm{Kg}$ of cement. This scenario assumes that $50 \mathrm{Kg}$ of cement is available on hand, and $60 \mathrm{Kg}$ is on order to be delivered on 07/15/2015. Table 34 presents one of the STREAM's outputs. It shows the tasks that are waiting for resources along the planning horizon, their eligible dates (the earliest date that all task predecessors are finished), the start date (the date the task is actually started), the waiting time (the difference in days between Start Date and Eligible Date), the needed resource, and the quantity that is lacking. For instance, task C2 was eligible to start at 6/29/2015, 
but it could not be started, even though all the required resources were available, except for the $50 \mathrm{~kg}$ of cement. Therefore, the task waited until 7/15/2015 to start. Whether or not there are non-renewable resources, STREAM can always warn about the required but unavailable resources and inform resulting task waiting times. This output is especially important in projects (constructions, shipyards, government, etc) where several resources (renewable or non-renewable) are required by individual tasks. Matching multiple resources for task execution is essential. Additionally, resources must be presented at commonly available periods in order to perform the tasks.

\section{Table 34: Task waiting for resources in scenario 7 (by STREAM)}

\begin{tabular}{|c|c|c|c|c|c|}
\hline Task & Eligible Date & Start Date & Waiting time (d) & Resource Needed & Qty. \\
\hline C2 & $\mathbf{6 / 2 9 / 2 0 1 5}$ & $\mathbf{7 / 1 5 / 2 0 1 5}$ & $\mathbf{1 5 . 6 2}$ & Cement & $\mathbf{5 0}$ \\
\hline C4 & $7 / 20 / 2015$ & $7 / 28 / 2015$ & 7.62 & Skilled Labor & 3 \\
\hline B4 & $7 / 27 / 2015$ & $8 / 27 / 2015$ & 30.62 & Skilled Labor & 1 \\
\hline E2 & $8 / 25 / 2015$ & $9 / 2 / 2015$ & 7.62 & Skilled Labor & 1 \\
\hline C5 & $8 / 26 / 2015$ & $9 / 3 / 2015$ & 7.62 & Skilled Labor & 2 \\
\hline B6 & $9 / 1 / 2015$ & $9 / 10 / 2015$ & 8.62 & Skilled Labor & 2 \\
\hline B5 & $9 / 1 / 2015$ & $9 / 3 / 2015$ & 1.62 & Skilled Labor & 2 \\
\hline C6 & $9 / 9 / 2015$ & $10 / 10 / 2015$ & 30.62 & Skilled Labor & 1 \\
\hline B7 & $10 / 9 / 2015$ & $11 / 17 / 2015$ & 38.62 & Skilled Labor & 1 \\
\hline C8 & $11 / 16 / 2015$ & $11 / 24 / 2015$ & 7.62 & Skilled Labor & 3 \\
\hline B8 & $11 / 23 / 2015$ & $12 / 17 / 2015$ & 23.62 & Skilled Labor & 1 \\
\hline C9 & $12 / 16 / 2015$ & $1 / 25 / 2016$ & 39.62 & Skilled Labor & 1 \\
\hline B10 & $1 / 22 / 2016$ & $2 / 2 / 2016$ & 10.62 & Skilled Labor & 2 \\
\hline D1 & $2 / 1 / 2016$ & $2 / 16 / 2016$ & 14.62 & Skilled Labor & 1 \\
\hline D5 & $2 / 26 / 2016$ & $3 / 7 / 2016$ & 9.62 & Concreting Labor & 1 \\
\hline
\end{tabular}

For scenario 7, TPD was 307 days and cost was $\$ 24,625,122$. If a project manager renegotiates with the supplier anticipation on the cement order delivery date, project duration and cost reductions can be obtained. For example, if the $60 \mathrm{Kg}$ of cement is delivered on 6/29/2015, TPD and cost would be 275 days and $\$ 14,347,972$, respectively. 


\section{Comparison of scenarios}

This case study demonstrate the advantages of simulating multiple scenarios, as opposed to providing a single solution. In practice, these solutions exist, but project managers do not have the luxury of evaluating such alternatives on a day-to-day basis.

The first two scenarios do no provide resource allocation flexibility, TPD and cost were 275 days and $\$ 14,347,972$, respectively. TPD and cost obtained by STREAM for Scenario 3 (when additional resources are allowed) were 185 days and $\$ 1,898,007$, respectively. If no additional resources can be added, scenarios 4, 5 and 6, provide a practical short-term solutions. Scenarios 4 and 5 provide better results when compared with the base schedule. Under scenario 6 (when no resource could be added but multiskills and multi-modes are considered), STREAM found the "best" solution. TPD is reduced from 275 days to 218 days (57 fewer days) and total project cost is reduced from $\$ 14,347,972$ to $\$ 6,460,206$, representing a cost reduction of nearly 50\%. Finally, the issue of non-renewable resource shortage which exists in real-life projects, is described in scenario 7. In this case, STREAM provided information that can contribute to avoid critical non-renewable resource shortages, e.g., the renegotiation of the critical resource resulted in $\$ 10,000,000$ savings in the overall project cost. STREAM generates outputs for each scenario in about 2 seconds on a standard laptop computer.

Table 35 summarizes total project duration and total project costs for the seven scenarios, whereas Figure 30 and 31 provide graphical representations of the data displayed in Table 35. Start date is a hypothetical project starting date. Project managers define it. STREAM calculated the finish date for each individual task under the given scenario. Table 35 summarizes TPD, start date, finish date, and cost for all scenarios of case study 1 . 


\begin{tabular}{|c|c|c|c|c|}
\hline \multicolumn{5}{|c|}{ Table 35: Total Project Duration and Total Cost for the Seven Scenarios } \\
\hline & & \multicolumn{3}{|c|}{ STREAM } \\
\hline Scenario & TPD & Start date & Finish date & Total Project Cost (USD) \\
\hline 1 & 275 & $5 / 28 / 2015$ & $02 / 25 / 2016$ & $\$ 14,347,972$ \\
\hline 2 & 275 & $5 / 28 / 2015$ & $02 / 25 / 2016$ & $\$ 14,347,972$ \\
\hline 3 & 185 & $5 / 28 / 2015$ & $11 / 27 / 2015$ & $\$ 1,898,007$ \\
\hline 4 & 224 & $5 / 28 / 2015$ & $1 / 5 / 2016$ & $\$ 7,923,455$ \\
\hline 5 & 255 & $5 / 28 / 2015$ & $2 / 5 / 2016$ & $\$ 10,855,420$ \\
\hline $\mathbf{6}$ & $\mathbf{2 1 8}$ & $\mathbf{5 / 2 8 / 2 0 1 5}$ & $\mathbf{1 2 / 3 0 / 2 0 1 5}$ & $\mathbf{\$ 6 , 4 6 0 , 2 0 6}$ \\
\hline 7 & 307 & $5 / 28 / 2015$ & $3 / 30 / 2016$ & $\$ 24,625,122$ \\
\hline
\end{tabular}

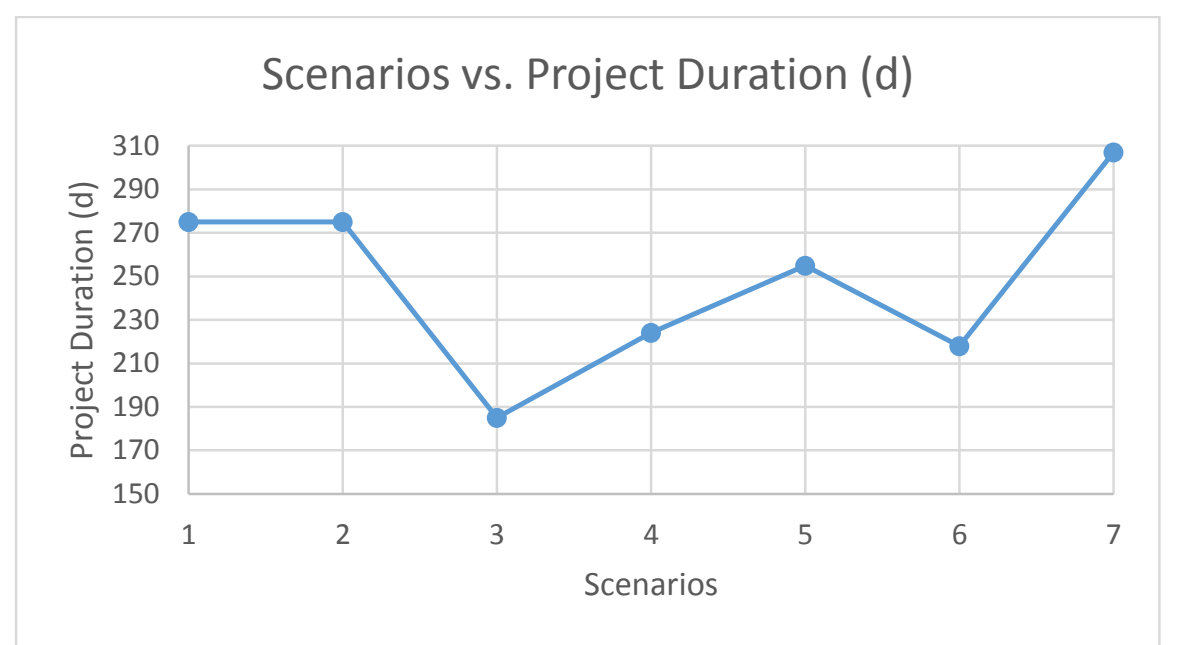

Figure 30: Project Duration (days) for Seven Scenarios

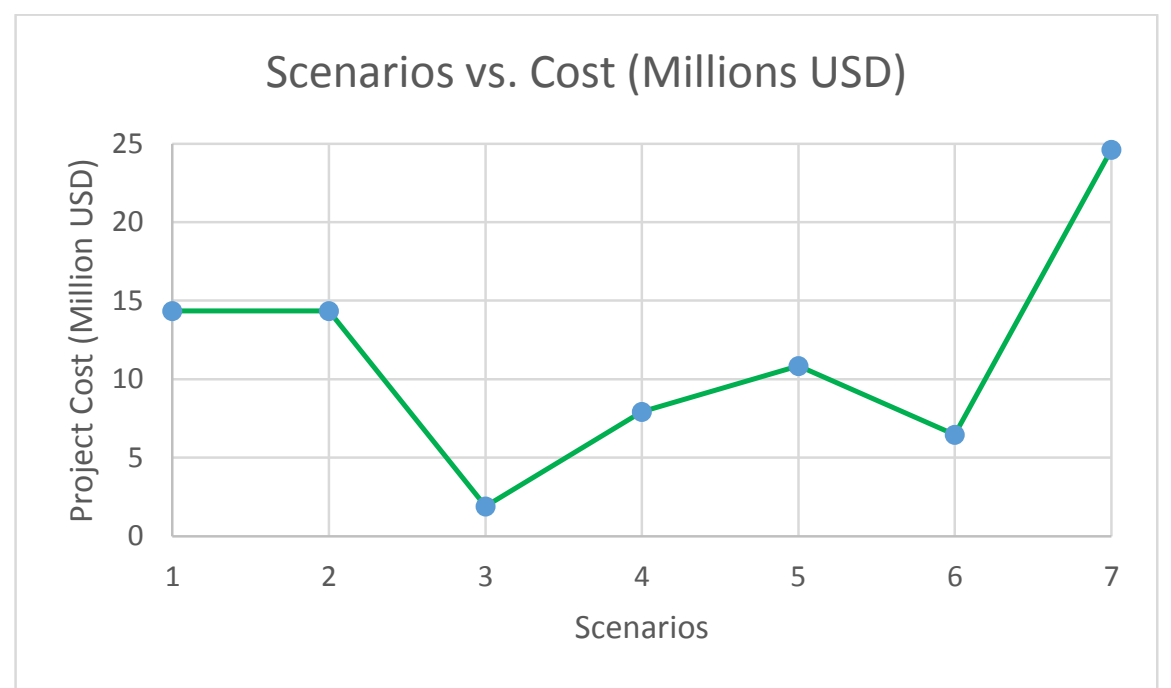

Figure 31: Project Cost (Millions USD) for Seven Scenarios 


\subsection{Case study 2: Oil Refinery Maintenance}

Refineries like other industries need to perform preventive maintenance, renovations, or upgrades on a regular basis. Refinery maintenance tasks are planned in order to service and to upgrade equipment and the reactor in a refinery. These procedures are carried out to ensure safe operations, stay competitive, and meet government regulations. The refinery usually shuts down part of the production during planned maintenance. Planned shutdowns are also known as turnarounds. Turnarounds can last for a week or a few months. Turnaround duration mainly depends on the project complexity [Kendrickoil, 2015].

\subsubsection{Inputs}

The details of an oil refinery turnaround project was described in Siu et al. (2015). It requested 107 tasks and 19 different types of resources. Limits of resources required to accomplish the 107 tasks are shown in Table 36. Table AIII.1 in the Appendix III describes the tasks, their durations, predecessors and their resource requirements, e.g. Task 1 requires two Boilermakers and two Boilermaker welders.

Table 36: Resource Description (Siu et. al, 2015)

\begin{tabular}{|c|c|l|c|}
\hline & Resource & \multicolumn{1}{|c|}{ Description } & Limits \\
\hline 1 & A & Boilermaker & $0-20$ \\
\hline 2 & B & Boilermaker welder & $0-10$ \\
\hline 3 & C & MSG80 3,600 t & $0-2$ \\
\hline 4 & D & Rigger & $0-7$ \\
\hline 5 & E & Scaffolder & $0-10$ \\
\hline 6 & F & Iron worker & $0-10$ \\
\hline 7 & G & Sterling 130 t crane & $0-6$ \\
\hline 8 & H & Technical & $0-5$ \\
\hline 9 & I & Inspection & $0-2$ \\
\hline 10 & J & Complex process operator & $0-2$ \\
\hline 11 & K & Refractory & $5-15$ \\
\hline 12 & L & Liquid penetrant inspection & $0-1$ \\
\hline 13 & M & X-Ray & $0-1$ \\
\hline 14 & N & PAUT inspection & $0-1$ \\
\hline 15 & O & Painter & $0-4$ \\
\hline 16 & P & Pipefitter & $0-5$ \\
\hline 17 & Q & Pipefitter welder & $0-4$ \\
\hline 18 & R & Inspector & $0-2$ \\
\hline 19 & S & Supervisor & $0-2$ \\
\hline
\end{tabular}




\subsubsection{Case study 2: Mathematical formulation by Siu et al. (2015)}

Siu et al. (2015) formulated the turnaround project with the objective to find the shortest total project duration, the earliest finish time for each task and the minimal resource requirements. They formulated the problem as optimization problem and use strong mathematical formulation. The optimal resource requirements were identified between the lower and upper bounds of resource supply limits. The objective function defined by Siu et al. (2015) was:

$$
\text { minimize } \mathrm{f}=\underbrace{\sum_{\text {task }} \sum_{\text {t=0 }}^{\mathrm{T}}\left(\alpha_{\text {Task }}^{\mathrm{t}} \mathrm{tx}_{\text {task }}^{\mathrm{t}}\right)}_{\text {i) Task completion times }}+\underbrace{\sum_{\mathrm{t}=0}^{\mathrm{T}}\left(\beta_{\text {proj }}^{\mathrm{t}} \mathrm{tx}_{\text {proj }}^{\mathrm{t}}\right)}_{\text {ii) project completion time }}+\underbrace{\sum_{\text {Res }} \gamma_{\text {Res }} \mathrm{R}_{\text {Res }}}_{\text {iii) Resource supply }}
$$

The objective function consists of three parts: i) task completion times, ii) project completion time, and iii) resource supply. Parameter $\alpha_{\text {task }}^{\mathrm{t}}$ is the relative importance of completion time of particular task at time t; parameter $\beta_{\text {Proj }}^{\mathrm{t}}$ is the relative importance of completion time of the project at time $\mathrm{t}$; and parameter $\gamma_{\text {Res }}$ is the relative importance of supply quantity for particular resource. Variable $\mathrm{x}_{\text {task }}$ is a binary variable, 1 represents a task completion at time $t$, or 0 otherwise; $x^{\mathrm{t}}$ Proj is a binary variable, 1 represents a project completion at time $t$, or 0 otherwise; $\mathrm{R}_{\mathrm{Res}}$ is a supply limit of particular resource. Siu et al. (2015) also defines the task precedence and resource limits constraints.

Siu et al. (2015) proposed a two-stage optimization solution. The first stage is to determine optimum project duration (shortest duration) and optimum resource supply (minimal quantity of resources). The second stage, given the analytical solutions resulting from the first stage, is designed to minimize the task finish times. The first stage is performed by setting the parameters $\left\{\alpha_{\text {task }}^{\mathrm{t}}, \beta_{\text {Proj }}^{\mathrm{t}}\right.$, $\left.\gamma_{\text {Res }}\right\}$ as $\left\{0, \beta_{\text {Proj }}^{\mathrm{t}}, \gamma_{\text {Res }}\right\}$, whereas in the second stages the parameters are as $\left\{\alpha_{\text {task }}^{\mathrm{t}}, 0,0\right\}$.

The objective was to determine the schedule which provides the shortest total project duration, the earliest finish time for each task, and utilizes minimum resources. Parameters $\left\{\alpha_{\text {task }}^{\mathrm{t}}, \beta^{\mathrm{t}}{ }_{\text {Proj }}, \gamma_{\mathrm{Res}}\right\}$ are 
assumed known. No resource calendars were utilized and it was assumed that all resources work 7 days a week, 24 hours a day. Case of multi-modes, multiple calendars, and multiple skills were not modelled.

Siu et al. (2015) used Matlab to solve the turnaround project. The optimum resource requirements and total project duration determined by Siu et al. (2015) is shown in Table 37. Table AIII.2 in the Appendix III shows the earliest finish time for each task, 0 is used as the project starting time.

Table 37: Optimal total project duration and resource requirements (Siu et. al, 2015)

\begin{tabular}{|c|c|}
\hline Resource & Optimal resource requirements \\
\hline $\mathrm{A}$ & 14 \\
\hline $\mathrm{B}$ & 9 \\
\hline $\mathrm{C}$ & 1 \\
\hline $\mathrm{D}$ & 6 \\
\hline $\mathrm{E}$ & 6 \\
\hline $\mathrm{F}$ & 6 \\
\hline $\mathrm{G}$ & 6 \\
\hline $\mathrm{H}$ & 2 \\
\hline $\mathrm{I}$ & 2 \\
\hline $\mathrm{J}$ & 2 \\
\hline $\mathrm{K}$ & 7 \\
\hline $\mathrm{L}$ & 1 \\
\hline M & 1 \\
\hline $\mathrm{N}$ & 1 \\
\hline $\mathrm{O}$ & 2 \\
\hline $\mathrm{P}$ & 2 \\
\hline Q & 1 \\
\hline $\mathrm{R}$ & 1 \\
\hline $\mathrm{S}$ & 1 \\
\hline Total Project Duration (TPD) & 173 (hours) \\
\hline
\end{tabular}

\subsubsection{Case study 2: STREAM formulation}

The outputs generated by STREAM for this case study included: i) Task waiting times, ii) Lack of resources when needed and iii) Resource utilization. The outputs can support project managers to reduce project duration, to find the earliest finish time for tasks and to determine the minimal resource requirements. Task waiting times output assists project managers to identify how long a given task will be waiting for resources. Lack of resources output helps project managers determine which resources are not 
available when a given task requires them. Resource utilization output shows how individual resources are utilized over the entire project duration. These three STREAM's outputs empower project managers in obtaining good and sometimes optimal solutions. In additional the optimal solution, STREAM provides alternate solutions to the project managers. Project manager has the option to deploy non-optimal feasible solution during project duration.

STREAM does not utilize the three parameters, $\alpha_{\text {task }}^{\mathrm{t}}, \beta_{\text {Proj }}^{\mathrm{t}}$, and $\gamma_{\text {Res }}$. STREAM was able to find the optimal solution to the oil refinery turnaround project without mathematically formulating it as an optimization problem. In addition, STREAM provided critical information to the project manager.

For the turnaround project case study, a rule which prioritizes a given task which has the largest number of successors is applied dynamically, at different point in time. This rule enables STREAM to minimize delay to least number of tasks. By applying this rule, STREAM aims to deliver a solution targeting the first and third goals (shortest project duration and earliest task finish times). STREAM also meets the goal to utilize least possible amount of resources. E.g., if 20 boilermakers exist in the resource pool, it does not imply that STREAM will assign all of them. STREAM assigns resources such that resources conflicts among tasks are avoided. If more than 20 boilermakers are required for tasks, some tasks will have to wait until the boilermakers are available. The same logic is applied for other resources. This procedure is directly related to the second goal (optimum resource supply).

The case study 2 has a unique scenario with the objective to determine the schedule which provides the shortest total project duration, the earliest finish time for each task, and utilizes minimum resources. Different simulation runs were conducted to demonstrate STREAM's ability to find diverse solutions. The results are validated with the baseline results (Tables 37 and AIII.2 in the Appendix III) provide by Siu et al. (2015). Four different simulation runs were carried out for the turnaround project. Details of the four simulation runs are as follows: 
Run 1: Determine the shortest total project duration, the earliest finish time for each task, and the minimal quantity of resources. Table 36 shows resource supply limits, task precedence and duration are shown in Table AIII.1 in the Appendix III. No resource calendars were used in order to compare results with Siu et al. (2015).

Run 2: Same as run 1 (data and goals), but priorities were changed for two tasks based on the waiting time for tasks output. STREAM finds values for the three unknown parameters $\left\{\alpha_{\text {task }}^{\mathrm{t}}, \beta_{\text {Proj }}^{\mathrm{t}}\right.$, $\left.\gamma_{\text {Res }}\right\}$.

Run 3: Determine the shortest duration project and the minimal quantity of resources (Definition of Stage 1 by Siu et al. 2015). In this run, resource limits were changed based on resource utilization output calculated by STREAM in run 2

Run 4: Determine the earliest finish time for each task (Definition of Stage 2 by Siu et al. 2015 but increasing in resources are allowed). STREAM determined the earliest finish time for each task by eliminating any waiting time for tasks to be started. Resource supply limits are increased based on waiting time for tasks output, and the three parameters $\left\{\alpha_{\text {task }}^{\mathrm{t}}, \beta_{\text {Proj }}^{\mathrm{t}}, \gamma_{\mathrm{Res}}\right\}$ are known based on run 2 results.

\subsubsection{Results applying STREAM}

Total project duration time computed by STREAM was 173 hours. It is same as the one found by Siu et al. 2015 .

\section{Simulation run \#1}

In run 1, STREAM was able to finish 103 tasks exactly at the same finish time as in Siu et al. 2015. Only four tasks $(93,96,101$, and 103) finished at different times. STREAM finished tasks 93 and 96 in 3, and 6 hours, respectively, whereas Siu et al. 2015's finished them in 5 and 8 hours, respectively. STREAM finished task 101 and 103 in 5 and 8 hours, whereas in Siu et al. 2015 they finished in 3 and 6 hours respectively. Table AIII.3 in the Appendix III shows in detail finish times for each task along with a comparison with baseline. It is clear from the results that STREAM found a different optimal solution. 
Tasks 93 and 96 were finished by STREAM two hours earlier than in Siu et al. 2015, whereas 101 and 103 were finished two hours later. Both methods provided the optimal solution for the turnaround project based on the objective function defined by Siu et al. (2015). STREAM does not explicitly search for an optimal solution. However, the results obtained by STREAM were comparable to those by Siu et al. (2015), who formulated the turnaround problem an optimization problem.

For simulation run 1, the task waiting times for required resources are shown in Table 38. Notice, the four tasks described are the only ones without a perfect task flow. Perfect task flow is when the task becomes eligible to start, and all its required resources are available and assigned to the task, without any waiting time. The four tasks described are those which may offer opportunities to improve the solution. This output was not provided by Siu et al. (2015). The ability to show which task will wait for resource is very valuable for the project manager. Manager can then plan for such event.

\section{Table 38: Task waiting for resources (STREAM Run1)}

\begin{tabular}{|c|c|c|c|c|c|}
\hline Task & Eligible Date & Start Date & Waiting time (h) & Resource Needed & Qty. \\
\hline 93 & $5 / 28 / 20150: 00$ & $5 / 28 / 20152: 00$ & 2 & Inspection & 1 \\
\hline 94 & $5 / 28 / 20150: 00$ & $5 / 28 / 20153: 00$ & 3 & Inspection & 1 \\
\hline 101 & $5 / 28 / 20150: 00$ & $5 / 28 / 20154: 00$ & 4 & Inspection & 1 \\
\hline 102 & $5 / 28 / 20150: 00$ & $5 / 28 / 20151: 00$ & 1 & Complex process operator & 1 \\
\hline
\end{tabular}

In Table 38, the date $05 / 28 / 2015$ is the hypothetical turnaround project start date. Eligible date is the date on which a task becomes eligible to start. The difference between start and eligible dates is the waiting time due to a lack of resource when needed. The lack of resource and its quantity are also described in Table 38. Task 93 is eligible to start at the beginning of the simulation (5/28/2015 0:00), however it can only be started 2 hours later due to the lack of resource. One resource (Inspection) is lacking and it becomes available only at 5/28/2015 2:00. The same logic is used for the other three tasks described in Table 38. STREAM was able to find a different optimal solution in run 1 with the goal of finding the shortest project duration, optimum resource supply, and earliest task finish times. 
The line curve in Figure 32 represents the finish time for each individual task calculated by the mathematical formulation in Siu et al. (2015) (baseline schedule). The line curve was constructed with the finish times displayed in Table AIII.2 in the Appendix III. The shaded region represents the finish times calculated by STREAM. Tasks 93 and 96 were finished 2 hours earlier, whereas Tasks 101 and 103 were finished 2 hours later than the baseline schedule. Table AIII.3 in the Appendix III displays the data.

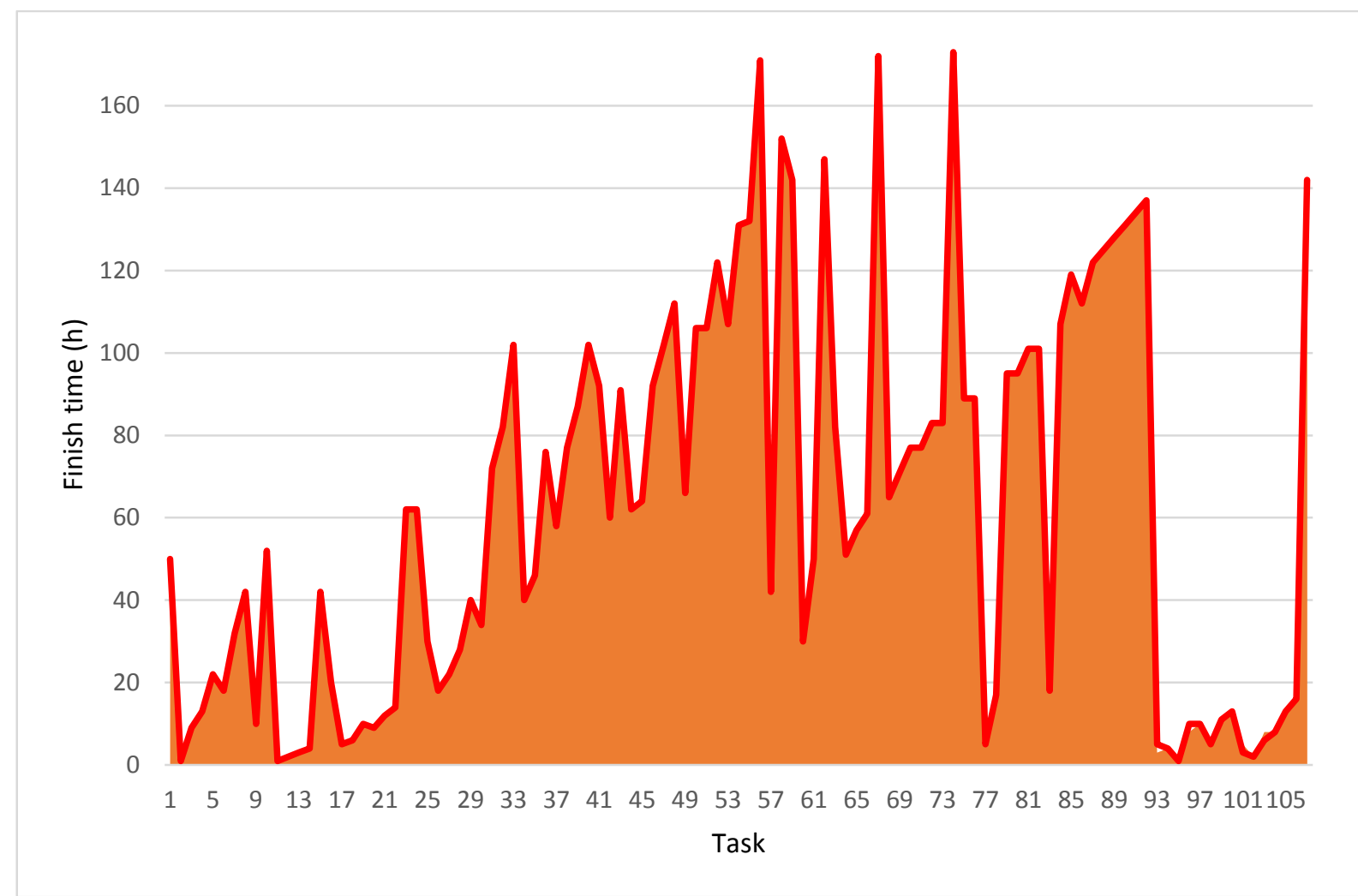

Figure 32: Task finish times for Siu et al., 2015 and STREAM Run 1

\section{Simulation Run \#2}

Based on the outputs generated by run \# 1, priorities for Tasks 101 and 93 were changed to 1000, and 400, respectively. STREAM utilizes a priority scale of 1-1000, with 1 being the lowest priority and 1000 the highest priority. By default all priorities are set to 500. Table 39 shows that the waiting times of Task 101 and 93 were changed based on the new priorities assigned. The required resources which are not available when required are also shown in Table 39. 
Table 39: Task waiting for resources (STREAM Run 2)

\begin{tabular}{|c|c|c|c|c|c|}
\hline Task & Eligible Date & Start Date & Waiting time (h) & Resource Needed & Qty. \\
\hline 93 & $5 / 28 / 20150: 00$ & $5 / 28 / 20154: 00$ & 4 & Inspection $(\mathrm{I})$ & 1 \\
\hline 94 & $5 / 28 / 20150: 00$ & $5 / 28 / 20153: 00$ & 3 & Inspection & 1 \\
\hline 101 & $5 / 28 / 20150: 00$ & $5 / 28 / 20152: 00$ & 2 & Inspection & 1 \\
\hline 102 & $5 / 28 / 20150: 00$ & $5 / 28 / 20151: 00$ & 1 & Complex process operator & 1 \\
\hline
\end{tabular}

Figure 33 shows the resource utilization for the data described in Table AIII.4 in the Appendix III. Figure 33 shows the resource utilization graphs for each individual team. Resource group A (Boilermakers) and B (Boilermaker welders) have the highest utilization.

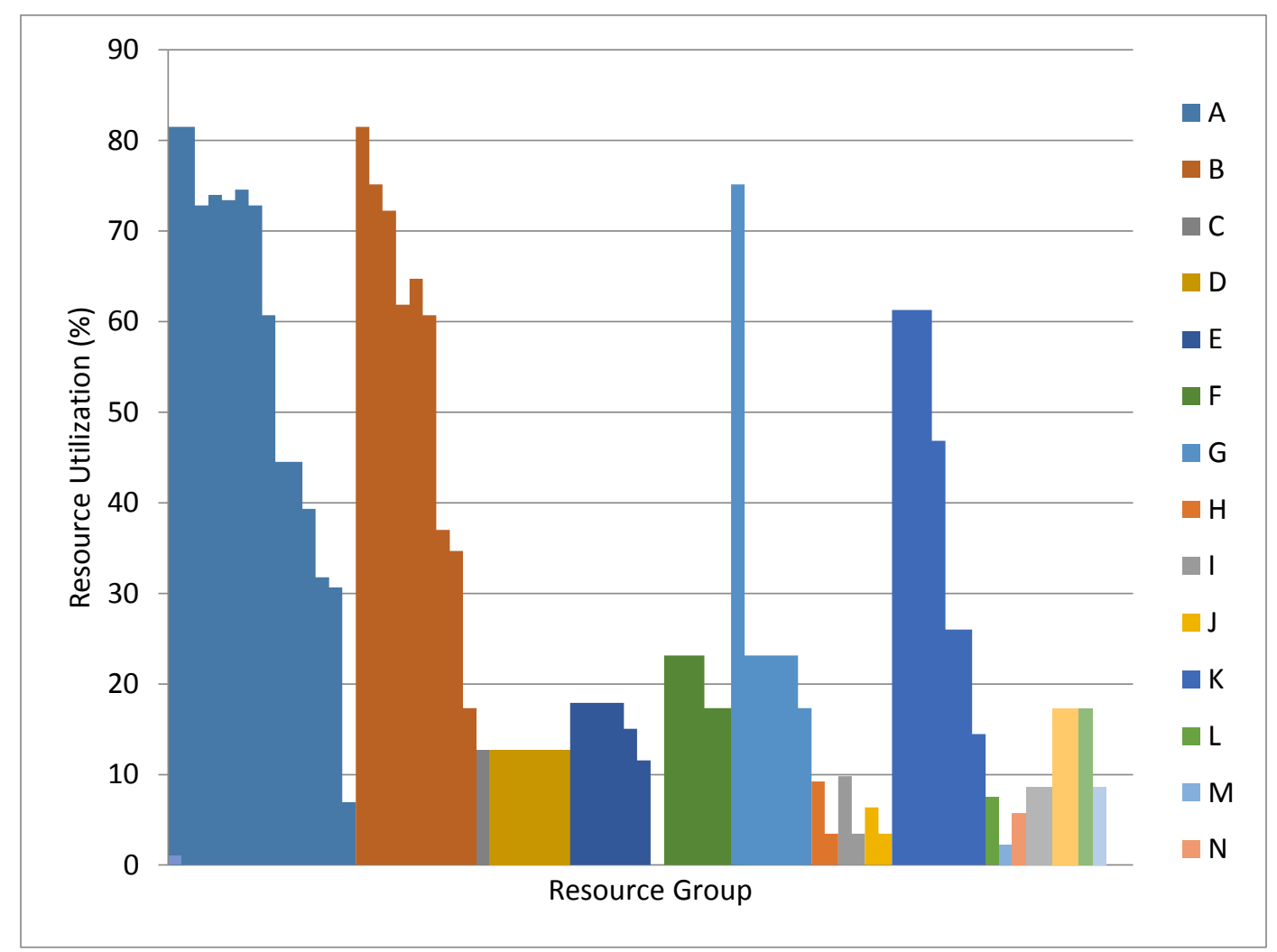

Figure 33: Resource utilization

The line curve in Figure 34 represents the finish time for each individual task calculated by the strong formulation in Siu et al. (2015). The line curve was constructed with the finish times displayed in Table AIII.2 in the Appendix III. The green shaded region represents the finish times calculated by 
STREAM. Table AIII.3 in the Appendix III displays the data. None of the finish times calculated by STREAM were greater than the optimal solution. Thus, the green shaded region does not go beyond the line curve.

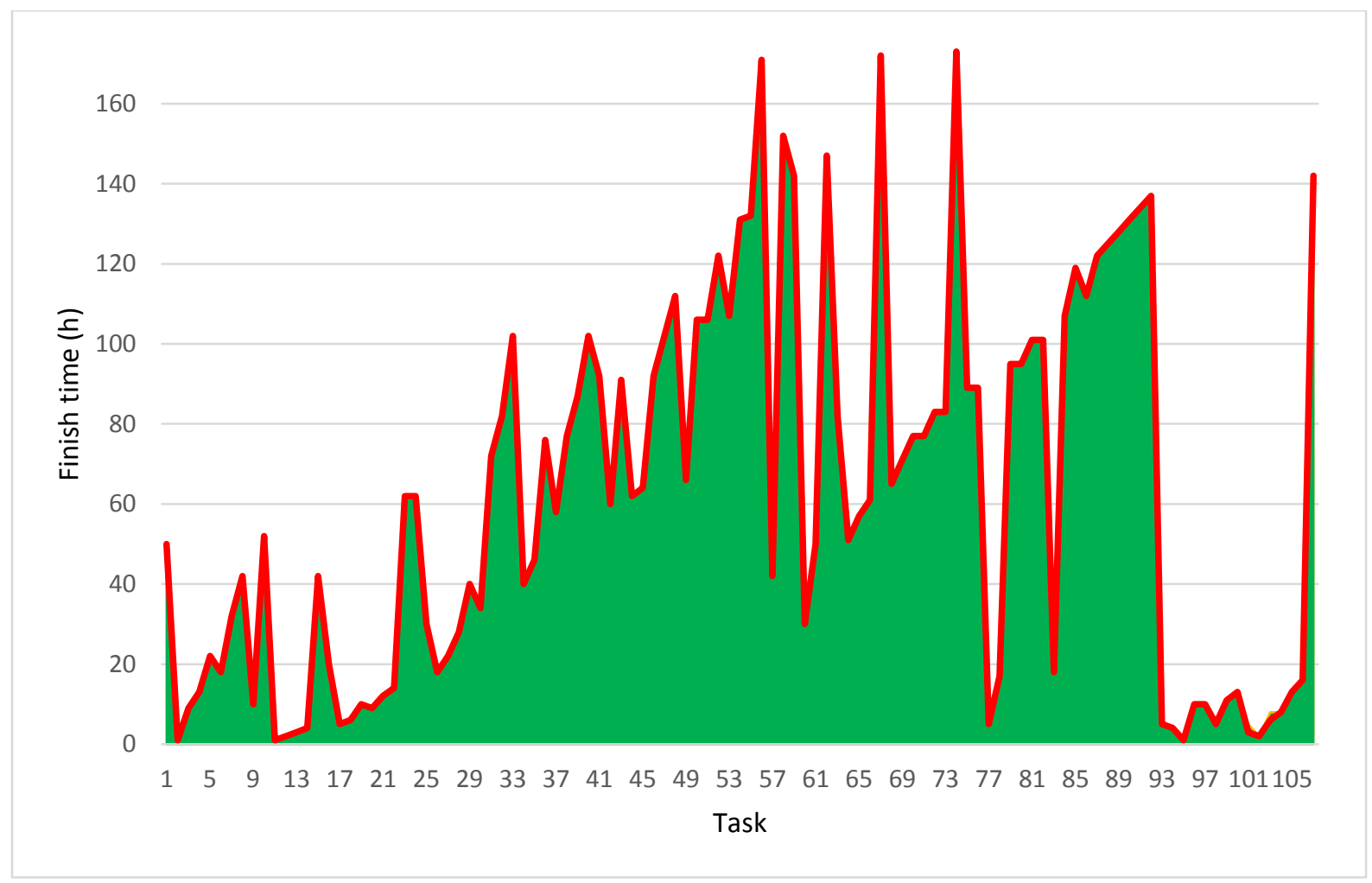

Figure 34: Task finish times for Siu et al., 2015 and STREAM Run 2

\section{Simulation Run \#3}

In simulation run 3, STREAM attempts to minimize resource requirements while keeping project duration time constant to 173 hours. In order to do so, the resource utilization provided by STREAM in simulation run 2 is used. Resources were found to have low utilization (Table AIII.4 in the Appendix III), the 14th boilermaker (A) has only $6.94 \%$ of utilization duration the simulation. The other 13 boilermakers have higher utilization about $30 \%$. This is an indication that 14 boilermakers may not be necessary to deliver the project in 173 hours, and one boilermaker can be reduced. The same logic is applied for other types of resources. The quantity of resource used for each type was defined by STREAM during the simulation run 2. The resource supply limits were the same as shown in Table 36, however STREAM has 
required the optimal resource supply. STREAM will required resources to finish tasks as earlier as possible. Resource types C, M, N, Q, R, and S were required only one unit for each type. Run 3, therefore, represents a case where resource supply limits are even more constrained while keeping the shortest project duration constant. Table 40 shows the resource requirements for run \# 3. STREAM reduced the number of Boilermakers (A) from 14 to 13, number of Iron workers (F) and Sterling $130 \mathrm{t}$ crane $(\mathrm{G})$ was reduced from 6 to 5 , number of technical $(\mathrm{H})$, inspection (I), and complex process operator (J) were reduced from 2 to 1 . Any reduction beyond of these limits will result in increasing the TPD. Table AIII.3 in the Appendix III shows the task finish times respectively for simulation run 3. It was expected that with fewer resources, some task finish times will increase. The task waiting times and required resources which are not available when needed are shown in Table 41 for simulation 3. It was also expected that with fewer resources, some waiting times will increase. The goal of STREAM is to provide several feasible options, it is left to the project manager to decide which option is appropriate for the current scenario.

\section{Table 40: Case of reduced resource (STREAM Run 3)}

\begin{tabular}{|c|c|l|c|}
\hline & Resource & \multicolumn{1}{|c|}{ Description } & Limits \\
\hline 1 & A & Boilermaker & 13 \\
\hline 2 & B & Boilermaker welder & 9 \\
\hline 3 & C & MSG80 3,600 t & 1 \\
\hline 4 & D & Rigger & 6 \\
\hline 5 & E & Scaffolder & 6 \\
\hline 6 & F & Iron worker & 5 \\
\hline 7 & G & Sterling 130 t crane & 5 \\
\hline 8 & H & Technical & 1 \\
\hline 9 & I & Inspection & 1 \\
\hline 10 & J & Complex process operator & 1 \\
\hline 11 & K & Refractory & 7 \\
\hline 12 & L & Liquid penetrant inspection & 1 \\
\hline 13 & M & X-Ray & 1 \\
\hline 14 & N & PAUT inspection & 1 \\
\hline 15 & O & Painter & 2 \\
\hline 16 & P & Pipefitter & 2 \\
\hline 17 & Q & Pipefitter welder & 1 \\
\hline 18 & R & Inspection & 1 \\
\hline 19 & S & Supervisor & 1 \\
\hline
\end{tabular}




\section{Table 41: Task waiting for resources (STREAM run 3)}

\begin{tabular}{|c|c|c|c|c|c|}
\hline Task & Eligible Date & Start Date & Waiting time (h) & Resource Needed & Qty. \\
\hline 102 & $5 / 28 / 20150: 00$ & $5 / 28 / 20152: 00$ & 2 & Technical & 1 \\
\hline 101 & $5 / 28 / 20150: 00$ & $5 / 28 / 20155: 00$ & 5 & Technical & 1 \\
\hline 95 & $5 / 28 / 20150: 00$ & $5 / 28 / 20151: 00$ & 1 & Technical & 1 \\
\hline 94 & $5 / 28 / 20150: 00$ & $5 / 28 / 20157: 00$ & 7 & Technical & 1 \\
\hline 93 & $5 / 28 / 20150: 00$ & $5 / 28 / 201510: 00$ & 10 & Technical & 1 \\
\hline 60 & $5 / 28 / 20150: 00$ & $5 / 29 / 201516: 00$ & 16 & Tron worker & 1 \\
\hline 12 & $5 / 28 / 20151: 00$ & $5 / 28 / 20153: 00$ & 2 & Technical & 1 \\
\hline 14 & $5 / 28 / 20155: 00$ & $5 / 28 / 20156: 00$ & 1 & Technical & 1 \\
\hline 17 & $5 / 28 / 20157: 00$ & $5 / 28 / 20158: 00$ & 1 & Boilermaker & 1 \\
\hline 100 & $5 / 28 / 201512: 00$ & $5 / 28 / 201513: 00$ & 1 & Boilermaker & 1 \\
\hline 21 & $5 / 28 / 201513: 00$ & $5 / 28 / 201514: 00$ & 1 & Sterling 130 t crane & 1 \\
\hline 10 & $5 / 28 / 201522: 00$ & $5 / 29 / 201516: 00$ & 18 & Boilermaker & 2 \\
\hline 61 & $5 / 29 / 201518: 00$ & $5 / 30 / 20152: 00$ & 8 & & \\
\hline
\end{tabular}

The line curve in Figure 35 represents the finish time for each individual task calculated by the mathematical formulation in Siu et al. (2015). The line curve was constructed with the finish times displayed in Table AIII.2 in the Appendix III. The shaded region represents the finish times calculated by STREAM. Table AIII.3 in the Appendix III displays the data. Some of the finish times calculated by STREAM were greater than the optimal solution. Tasks 70,73 , and 85 have finish times greater than the baseline schedule. Thus, the shaded region goes beyond the line curve. It is due to the intentional reduction of resources. 


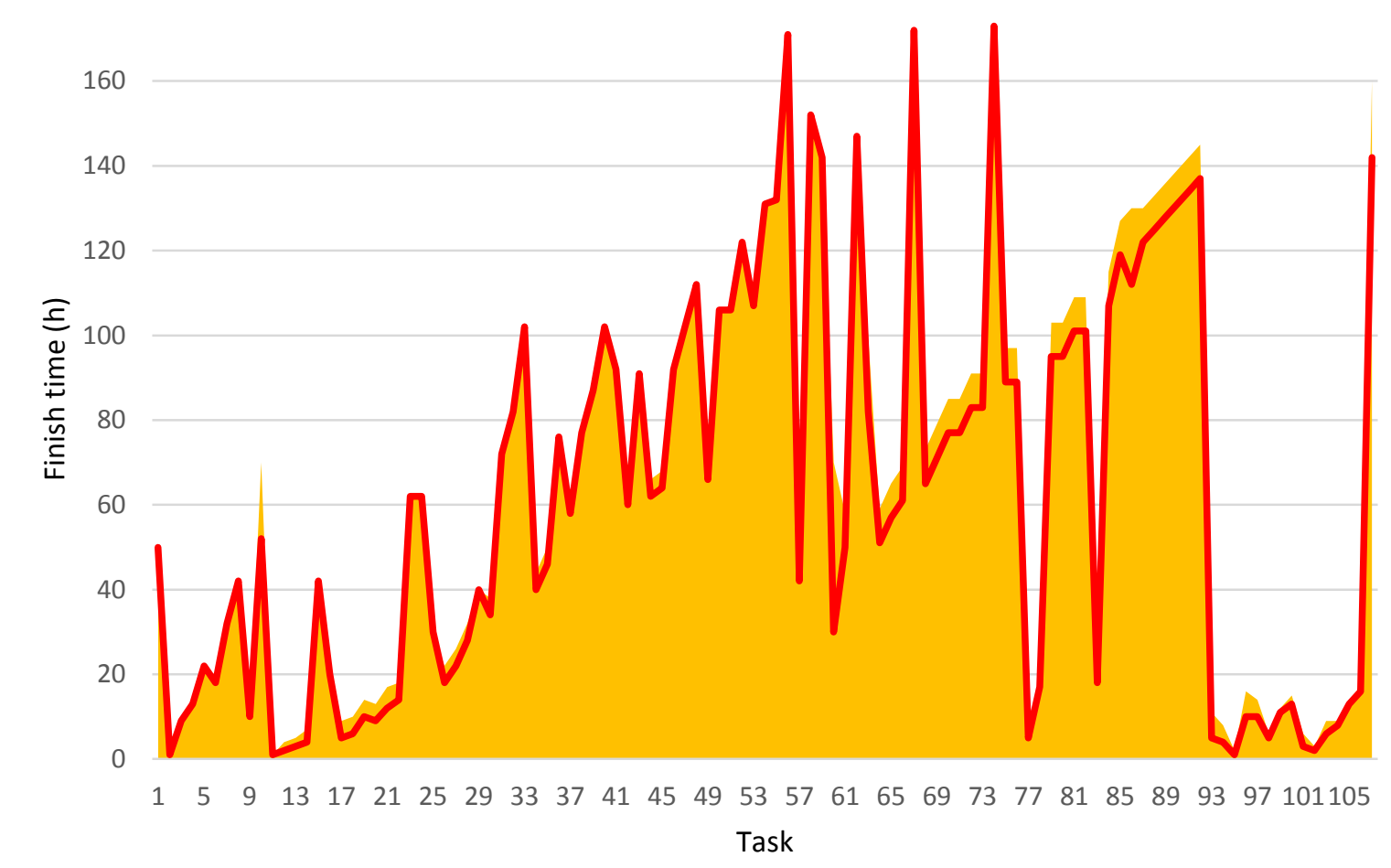

Figure 35: Task finish times for Siu et al., 2015 and STREAM Run 3

\section{Simulation Run \#4}

The simulation run 4 aims to find the perfect task flow among all the tasks. That is, no task waits due to resource unavailability. Ultimately, the goal is to find the earliest finish time for each individual task while the least resource requirement is found. No queue is created because once a given task becomes eligible, its required resources are available. In order to achieve this goal, two STREAM outputs needed are utilized: i) task waiting times, and ii) required resources which are not available when required. Simulation run 2 outputs is used in run 4 . Table 39 shows these outputs for simulation run 2 . At first glance 3 resources of inspection and 1 complex process operator should be added. However, when 3 resources of inspections (I) are added, needs for more technical $(\mathrm{H})$ and complex process operator $(\mathrm{J})$ come up simultaneously. Tasks 93, 94, 101 and 102 have the same resource requirements (H[1]; I[1]; J[1]) as shown in Table 37. Table 42 shows the resource requirements for run 4 only. STREAM increased the number of technical $(\mathrm{H})$, inspection (I), and complex process operator $(\mathrm{J})$ from 2 to 6 . Table 
43 shows the difference between simulation run 4 and the baseline results in terms of resource requirements. Table AIII.3 in the Appendix III shows which tasks have been benefit from the additional resources, e.g., Task 93, 94, 96, 97, 101, 102, 103, 104, 105, and 106 are finishing earlier 4, 3, 4, 3, 2, 1, 2, 1, 1, and 1 hours respectively. Additional resources do not affect task finish times and project duration time. STREAM was able to schedule all tasks without waiting times.

\begin{tabular}{|c|c|l|c|}
\hline \multicolumn{3}{|c|}{ Table 42: Case of increased resource (STREAM Run 4) } \\
\hline & Resource & \multicolumn{1}{|c|}{ Description } & Limits \\
\hline 1 & A & Boilermaker & 14 \\
\hline 2 & B & Boilermaker welder & 9 \\
\hline 3 & C & MSG80 3,600 t & 1 \\
\hline 4 & D & Rigger & 6 \\
\hline 5 & E & Scaffolder & 6 \\
\hline 6 & F & Iron worker & 6 \\
\hline 7 & G & Sterling 130 t crane & 6 \\
\hline 8 & H & Technical & 6 \\
\hline 9 & I & Inspection & 6 \\
\hline 10 & J & Complex process operator & 6 \\
\hline 11 & K & Refractory & 7 \\
\hline 12 & L & Liquid penetrant inspection & 1 \\
\hline 13 & M & X-Ray & 1 \\
\hline 14 & N & PAUT inspection & 1 \\
\hline 15 & O & Painter & 2 \\
\hline 16 & P & Pipefitter & 2 \\
\hline 17 & Q & Pipefitter welder & 1 \\
\hline 18 & R & Inspection & 1 \\
\hline 19 & S & Supervisor & 1 \\
\hline & & & \\
\hline
\end{tabular}

The line curve in Figure 36 represents the finish time for each individual task calculated by the mathematical formulation in Siu et al. (2015). The line curve was constructed with the finish times displayed in Table AIII.2 in the Appendix III. None of the finish times calculated by STREAM were greater than the optimal solution provided by Siu et al. (2015). Thus, the shaded region does not go beyond the line curve. Instead, some finish times in STREAM were smaller than the optimal solution. It is due to the addition of resources. 


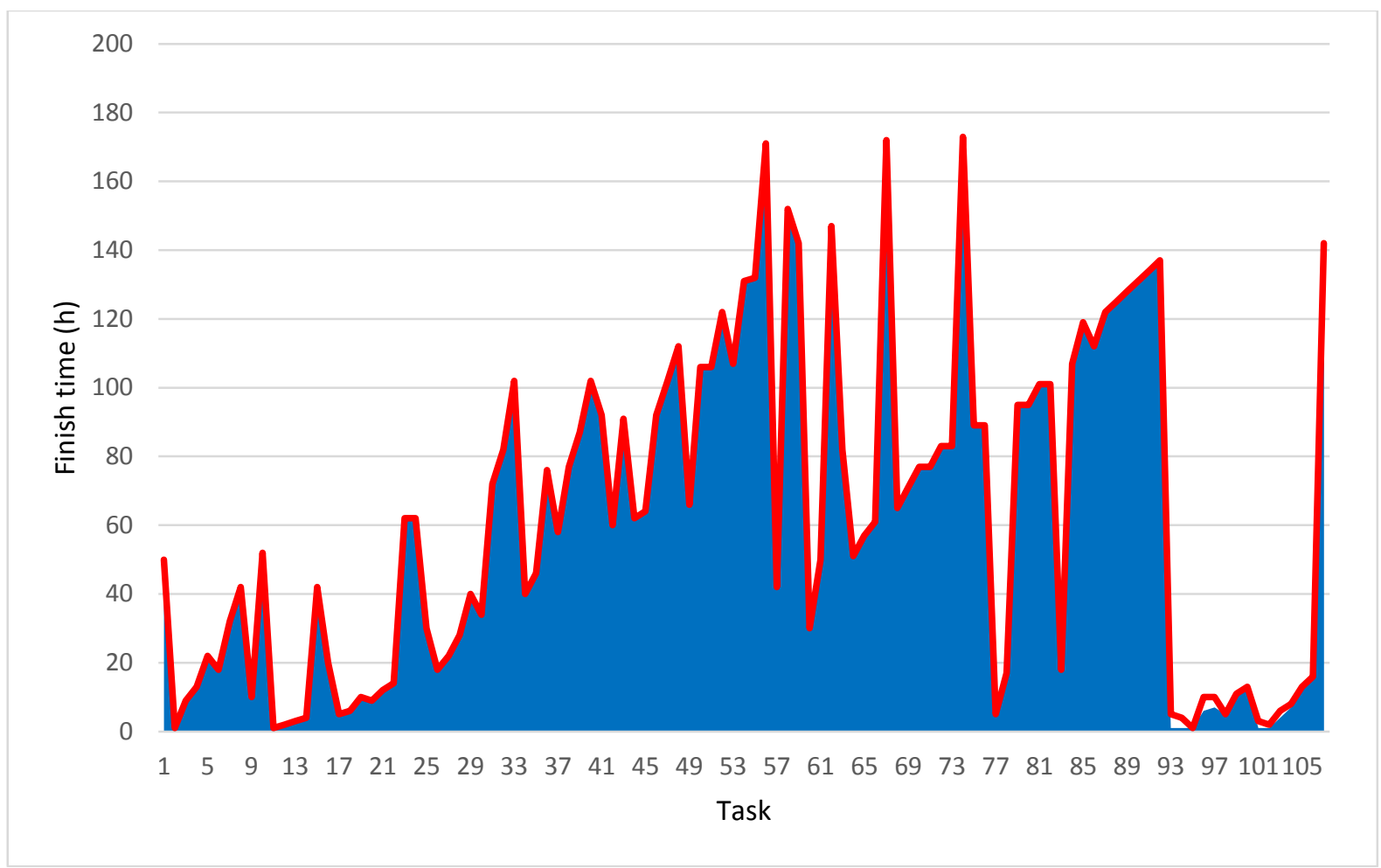

Figure 36: Task finish times for Siu et al., 2015 and STREAM Run 4

\section{Simulations results and discussion}

In runs 1 and 2, the optimal resource supply is also found as in Siu et al. (2015). Table 43 shows the baseline result (resource requirements) and validates it with the four simulation runs. The column Diff. in Table 43 represents the difference between STREAM and Siu et al. (2015) results. If it is 0 (zero) that means no difference was found, quantity of resource type A quantity in Siu et al. (2015) was 14 and STREAM also found 14 for runs 1 and 2.

STREAM was able to find the optimal solution in run 1 and run 2 by utilizing the two aforementioned STREAM outputs (Task waiting times and resource utilization). STREAM is not a "black box" for solving the optimization problems, where inputs come in and outputs are produced. STREAM is able to track in details what is happening during each simulation run. Notice that simulation runs 1 and 2 are optimal solutions, but knowing this information does not mean that tasks are not waiting for resources. Based on Tables 38, 39 and 41, there are tasks waiting for resources at different points in time. 
Therefore, this additional information provided by STREAM empowers project managers to find different solution under the optimal solutions. Tables 43 and Table AIII.3 in the Appendix III show the resource requirements and finish times for the four simulation runs, respectively.

\begin{tabular}{|c|c|c|c|c|c|c|c|c|}
\hline \multicolumn{9}{|c|}{ Table 43: Solution results } \\
\hline Resource & \multicolumn{2}{|c|}{ Run1 } & \multicolumn{2}{c|}{ Run 2 } & \multicolumn{3}{c|}{ Run 3 } & \multicolumn{2}{c|}{ Run 4 } \\
\hline & Qty. & Diff. & Qty. & Diff. & Qty. & Diff. & Qty. & Diff. \\
\hline A & 14 & 0 & 14 & 0 & 13 & -1 & 14 & 0 \\
\hline B & 9 & 0 & 9 & 0 & 9 & 0 & 9 & 0 \\
\hline C & 1 & 0 & 1 & 0 & 1 & 0 & 1 & 0 \\
\hline D & 6 & 0 & 6 & 0 & 6 & 0 & 6 & 0 \\
\hline E & 6 & 0 & 6 & 0 & 6 & 0 & 6 & 0 \\
\hline F & 6 & 0 & 6 & 0 & 5 & -1 & 6 & 0 \\
\hline G & 6 & 0 & 6 & 0 & 5 & -1 & 6 & 0 \\
\hline H & 2 & 0 & 2 & 0 & 1 & -1 & 6 & 4 \\
\hline I & 2 & 0 & 2 & 0 & 1 & -1 & 6 & 4 \\
\hline J & 2 & 0 & 2 & 0 & 1 & -1 & 6 & 4 \\
\hline K & 7 & 0 & 7 & 0 & 7 & 0 & 7 & 0 \\
\hline L & 1 & 0 & 1 & 0 & 1 & 0 & 1 & 0 \\
\hline M & 1 & 0 & 1 & 0 & 1 & 0 & 1 & 0 \\
\hline N & 1 & 0 & 1 & 0 & 1 & 0 & 1 & 0 \\
\hline O & 2 & 0 & 2 & 0 & 2 & 0 & 2 & 0 \\
\hline P & 2 & 0 & 2 & 0 & 2 & 0 & 2 & 0 \\
\hline Q & 1 & 0 & 1 & 0 & 1 & 0 & 1 & 0 \\
\hline R & 1 & 0 & 1 & 0 & 1 & 0 & 1 & 0 \\
\hline S & 1 & 0 & 1 & 0 & 1 & 0 & 1 & 0 \\
\hline
\end{tabular}


Figure 37 shows graphically the resource requirements for the four simulation runs and Siu et al. (2015) results. The line is the optimal resource requirement for resources obtained in Siu et al. (2015). Runs 1 and 2 obtained the optimal resource requirements, run 3 obtained fewer resources than the optimal solution, and run 4 more resources than the optimal solution.

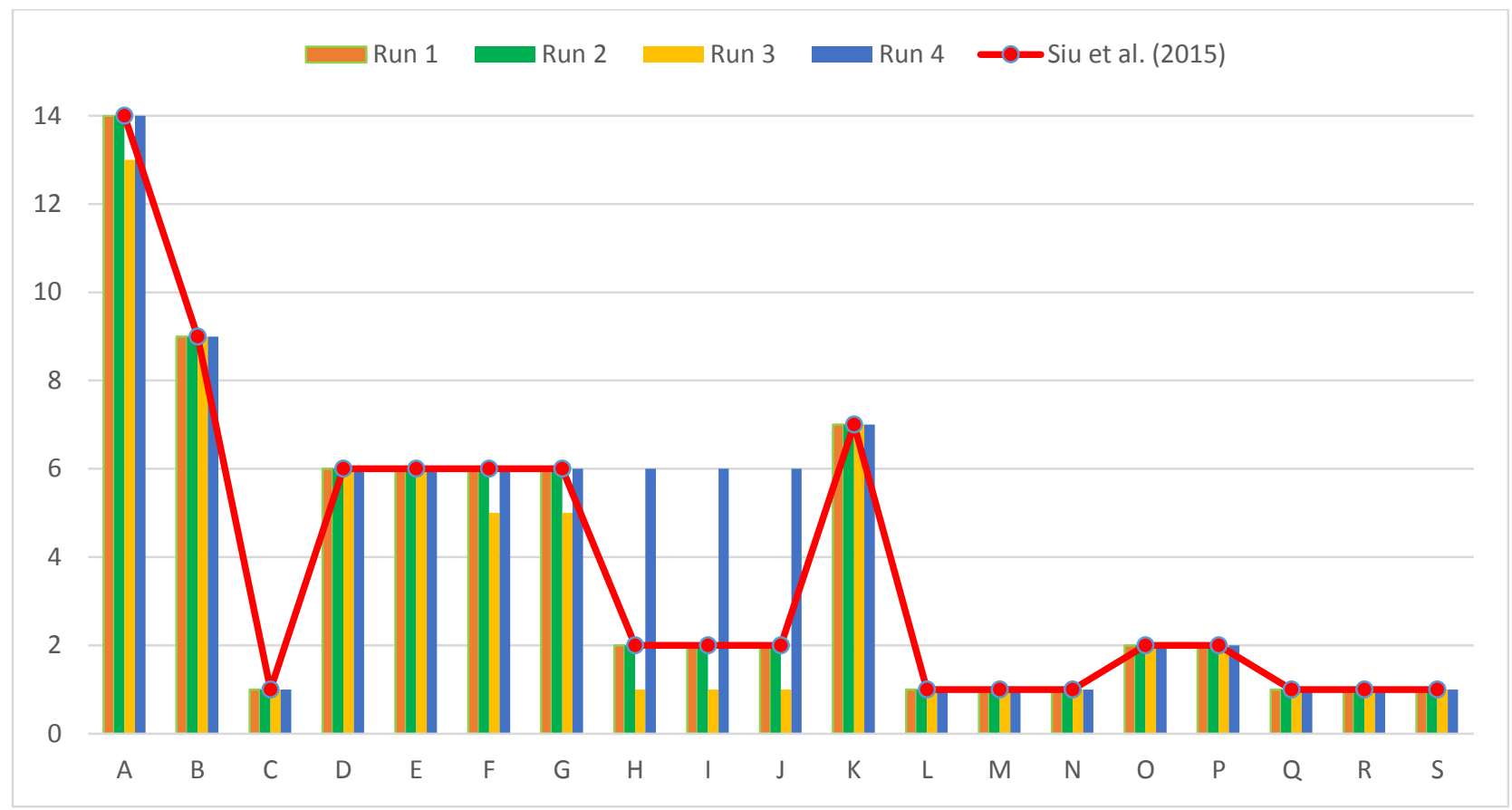

Figure 37: Resource requirements for Siu et al., 2015, STREAM Run 1, STREAM Run 2, and STREAM Run 3, STREAM Run 4 


\subsection{Case Study 3: Ship Repair}

Under this case study real world data was collected from the largest shipyard in the southern hemisphere. It is located at Rio de Janeiro/Brazil. Figure 38 shows an aerial view of the repair shipyard.

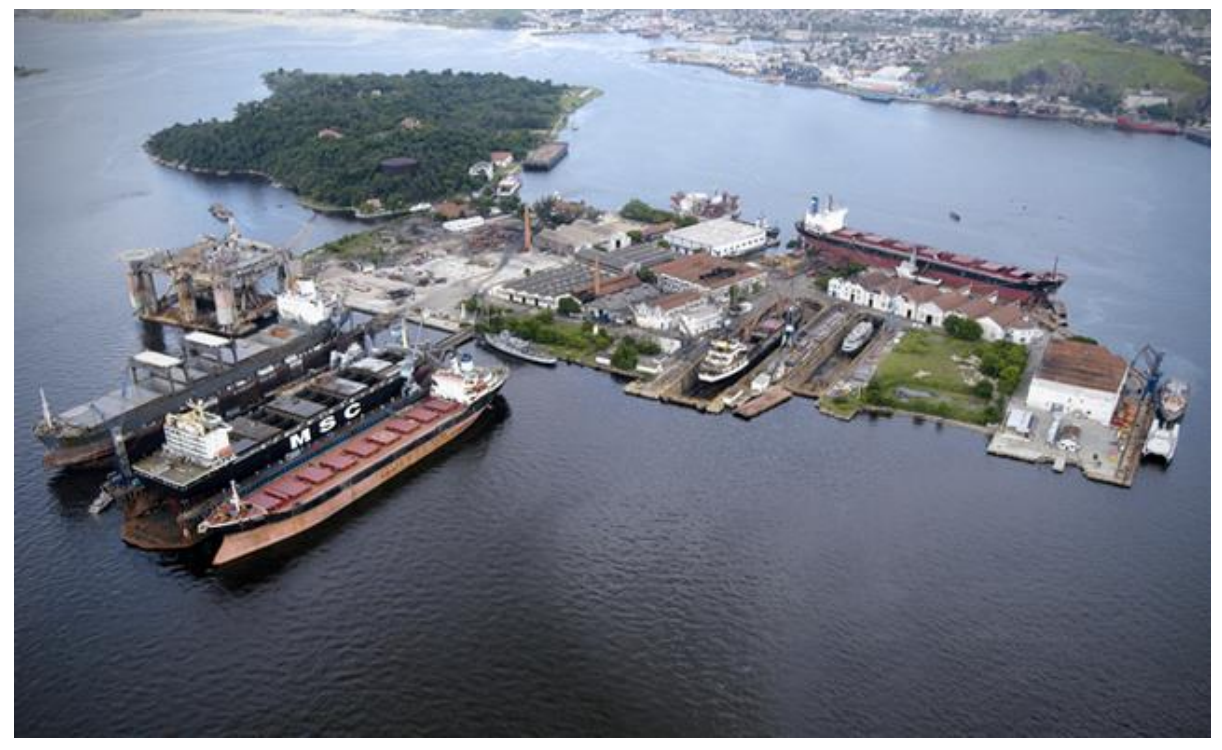

Figure 38: Repair shipyard facility located in Brazil

In the shipyard three floating docks and two dry docks. Docks are the most valuable resources of a repair shipyard, they are expensive and limited. Each dock is equipped with its own cranes. The cranes directly support nearly all the work teams as they move around the shipyard. In addition to the docks, cranes and work teams, the shipyard has a series of other production resources distributed throughout the facility. Some of these resources are allocated and grouped in resource centers. The grouping depends on the process utilized to carry out tasks. The metalworking resources are managed by metalworking centers; the tube and valve resources are located close to one another; the plasma cutting machinery is in a specific center, and so forth.

Docks could be occupied by more than one ship at a time. It is a favorable business situation, but it complicates the operations management. A manager is assigned for each dock. In Brazilian repair shipyards, the manager is in charge of all tasks related to her/his dock. Dock managers are highly skilled, 
and influential. The dock managers are rewarded for efficient operation of their docks. They schedule tasks on their docks using a simple spreadsheet or Microsoft Project software. They compete for finite resources with other dock managers. Such practice results in optimizing operations at an individual dock, while sub-optimizing the overall projects. Such an approach leads to scheduling slippage and cost overruns [Pinha and Ahluwalia, 2013], [Pinha and Ahluwalia, 2014], [Van Dijk, 2002]. In addition, there is lack of communication among stakeholders (dock managers, customers, suppliers, etc.). The system becomes dependent on dock mangers resourcefulness and skill level of the workforce, but system knowledge is not preserved. Therefore, a unified and centralized data for projects is required.

When a task is carried out at a shipyard, the necessary resources are sent to the desired location. The ships are static and the resources are brought to them. The key resources at a repair shipyard are the work teams and their tools. Typical work teams are: welders, mechanics, painters, and quality control personnel. As resources, the work teams are the key for project managers in the shipyard. Resource centers (metalworking, mechanical, tubing, etc.) respond to tasks needs. The bottlenecks occur not when the teams are working in the resource centers, but when they are working on board the ships, this being the factor that ultimately determines how long a ship remains in dock.

This production environment based on projects provides all necessary features for testing STREAM. All ships and offshore platforms, however large or small, undergo scheduled or unscheduled repair and maintenance. The bidding process for ship repair jobs is highly competitive and global in scope [Pinha and Ahluwalia, 2013], [Pinha and Ahluwalia, 2014]. The ship repair industry is also prone to risks due to high level of capital investment in facilities (such as dry docks), equipment (such as heavy duty cranes), and skilled work force [Pinha et al, 2011]. This industry provides highly customized service and deals with unpredictable demand [Dlugokecki et al., 2010]. Some aspects of ship maintenance, such as cleaning and painting have been automated [Sjøbakk et al., 2013], [Navarro et al., 2013], [Navarro et al., 2011]. However, a vast majority of tasks are performed manually. Typical services include: a) Docking, b) Hand scraping, c) High pressure fresh water jet cleaning, d) Painting, e) Tank cleaning, f) 
Steel work, g) Repair of ship's structure, h) Repair of propulsion system, i) Piping repair, j) Valve repair, k) Electrical system, 1) Undocking, and m) Testing at sea. Production planning and scheduling is difficult due to finite resources, such as docks, cranes, and worker skills and uneven flow of repair orders [Pinha and Ahluwalia, 2013], [Pinha and Ahluwalia, 2014], [Dlugokecki et al., 2010], [Mourtzis, 2005], [Wullink et al., 2004], [Van Dijk, 2002], [Chyrssolouris, 1999], [Chyrssolouris et al., 2004], [De Boer, 1998], [De Boer et al, 1997]. Table 44 lists some of the resources, grouped by work teams, machines, tools, and material handling devices [Pinha et al., 2011].

\begin{tabular}{|l|l|l|l|}
\hline \multicolumn{5}{|c|}{ Table 44: Types of Resources } \\
\hline \multicolumn{1}{|c|}{ Work Teams } & \multicolumn{1}{c|}{ Tools } & \multicolumn{1}{c|}{ Material Handling } \\
\hline Mechanical & Plasma Cutting & Hydro-jet pumps & Forklift \\
\hline Structure & Pipe bending & Paint pumps & Trucks \\
\hline Paint & Welding Machines & Hydraulic pumps & Cranes \\
\hline Sand-blasting & Tube resources & Sand-blasting pumps & Pulley \\
\hline
\end{tabular}

\subsubsection{Inputs}

A project for repairing a complex vessel is described. It is a class leading ROV (remotely operated underwater vehicle) construction support vessel ideally suited to perform subsea operations across a wide range of water depths and environmental conditions. It is used to repair oil offshore platforms. Figure 39 shows the vessel. The project was planned with 111 tasks with 475 multiple skilled resources in the resource pool.

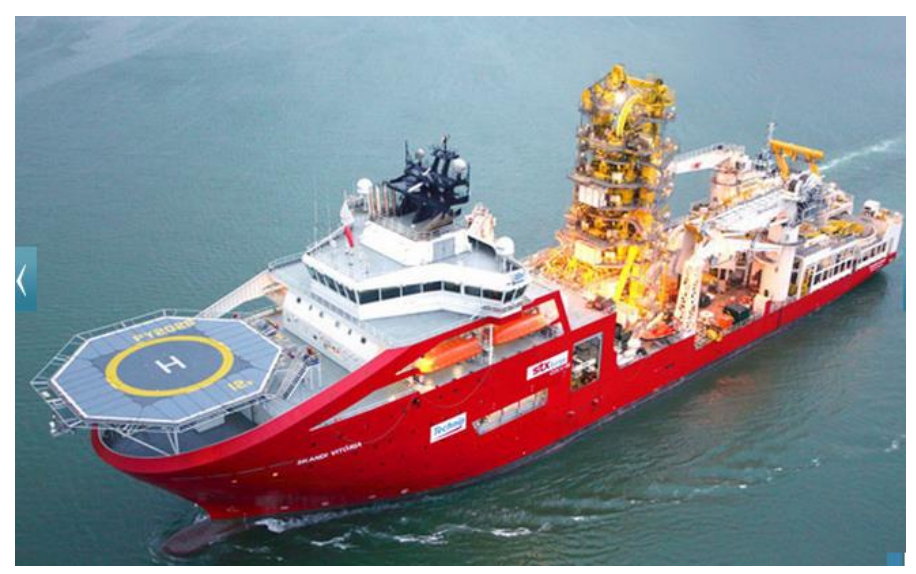

Figure 39: Vessel 
Table 45 describes only partially the required tasks, and their precedence relationship data. The complete data is presented in Table AIV.1 in the Appendix IV. Table 46 shows the skills required for repairing this ship. The resource requirements for tasks/modes, and the multi-skilled resources are described in Tables AIV.2 and AIV.3 respectively in the Appendix IV.

\begin{tabular}{|c|l|c|}
\hline & \multicolumn{1}{|c|}{ Table 45: Ship Repair Task Description } & \\
\hline Task & \multicolumn{1}{|c|}{ Description } & Pred. \\
\hline & Treatment and Painting & \\
\hline 1 & install scuppers pipes (top side) & 1 \\
\hline 2 & high pressure water (top side) & 2 \\
\hline 3 & treatment (top side) & 3 \\
\hline 4 & painting (top side) & 4 \\
\hline 5 & hull marks + name+ register port (top side) & \\
\hline$\cdot$ & & \\
\hline$\cdot$ & & \\
\hline$\cdot$ & & \\
\hline 108 & supply equipment for lifting 500 kg height min. 06 meters (life boats) & \\
\hline
\end{tabular}

\begin{tabular}{|c|l|}
\hline & \multicolumn{1}{|c|}{ Table 46: Skills } \\
\hline Skill & \\
\hline 1 & wescription \\
\hline 2 & blow torched \\
\hline 3 & brazier \\
\hline 4 & assembler \\
\hline 5 & mechanic \\
\hline 6 & electrician \\
\hline 7 & fluid jet \\
\hline 8 & painter \\
\hline 9 & plumber \\
\hline 10 & crane operator \\
\hline 11 & machine operator \\
\hline 12 & carrier \\
\hline 13 & assembler scaffolding \\
\hline 14 & carpenter \\
\hline 15 & dock aid \\
\hline 16 & support \\
\hline 17 & safety \\
\hline 18 & fireman \\
\hline 19 & general services \\
\hline 20 & quality control \\
\hline & \\
\hline
\end{tabular}


Figure 40 shows a screen where project managers can associate skills for a given resource in STREAM. Resource "worker 20" can work as welder, assembler and fireman in the shipyard.

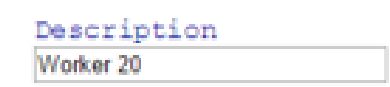

Ski11s

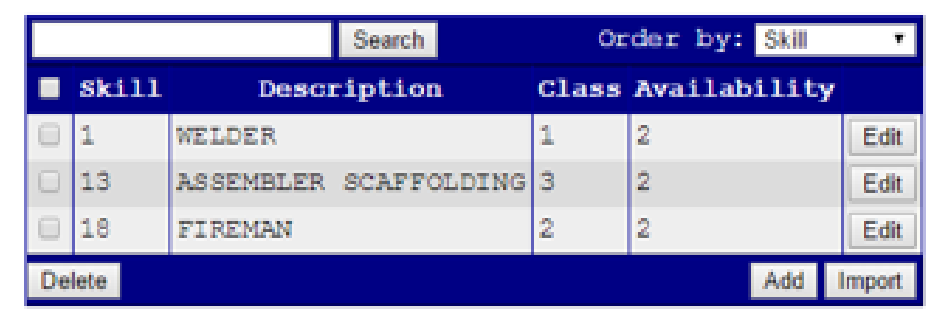

Figure 40: Multiple skills for a given resource in STREAM

\subsubsection{Results applying STREAM}

For this case study two simulations were run on a unique scenario. The first simulation was performed to assess which tasks are delayed due to lack of resources. The second run provides an improved result in light of outputs obtained for simulation run 1 . The waiting times for some tasks in the first run is shown in Table 47. These tasks are waiting for resources which were not available when required. One example in this case study is proposed. Task 61 was eligible to start on 03-Jun-2015, but 36 units of the nonrenewable resource (electrode ok 4600.1 / 8) were not available on hand. Task 61 was started on 30-Jun-2015. This was the date when a new order quantity of the nonrenewable resource was delivered in the shipyard. Simulation run 1 generates the outputs considering the nonrenewable resource issue. What-if the delivery date for the nonrenewable resource could be anticipated for 03-Jun-2015. Simulation 2 is run to assess this, and a comparison between simulations 1 and 2 is made. 
Table 47: Lack of resources when required (Run 1)

\begin{tabular}{|c|c|c|c|c|c|}
\hline Task & Eligible Date & Start Date & Waiting Time & Qty. & Resources \\
\hline 89 & 18-May-15 & 12-Jun-15 & 25.58 & 1 & Fluid Jet \\
\hline 88 & 18 -May-15 & 12-Jun-15 & 25.54 & 1 & Fluid Jet \\
\hline 82 & 18 -May-15 & 19-May-15 & 1.13 & 1 & Support \\
\hline 84 & $19-$ May-15 & 22-May-15 & 3.04 & 5 & Fluid Jet \\
\hline 35 & $19-$ May-15 & 01-Jun-15 & 13.04 & 14 & Fluid Jet \\
\hline 50 & $22-$ May-15 & 04-Jun-15 & 13.08 & 10 & Fluid Jet \\
\hline 43 & $22-M a y-15$ & 03-Jun-15 & 11.83 & 12 & Fluid Jet \\
\hline 68 & 28-May-15 & 08-Jun-15 & 10.62 & 1 & Valves repairing \\
\hline $\mathbf{6 1}$ & 03-Jun-15 & 30-Jun-15 & $\mathbf{2 6 . 9 2}$ & $\mathbf{3 6}$ & electrode ok 4600.1 / 8 \\
\hline 51 & 12-Jun-15 & 24-Jun-15 & 11.75 & 7 & Painter \\
\hline 21 & 18-Jun-15 & 19-Jun-15 & 0.92 & 1 & Painter \\
\hline
\end{tabular}

Figure 41 shows the resource utilization bar chart for all required resource for repairing the vessel. $\mathrm{X}$-axis represent resources, $\mathrm{y}$-axis is the resource utilization which can vary from $0 \%$ to $100 \%$. The colors for bars in the chart represent skills. E.g., red color bars are resources for painting, yellow are resources for plumbing, and so on. The chart provides a clear indication that painters are the resource most utilized.

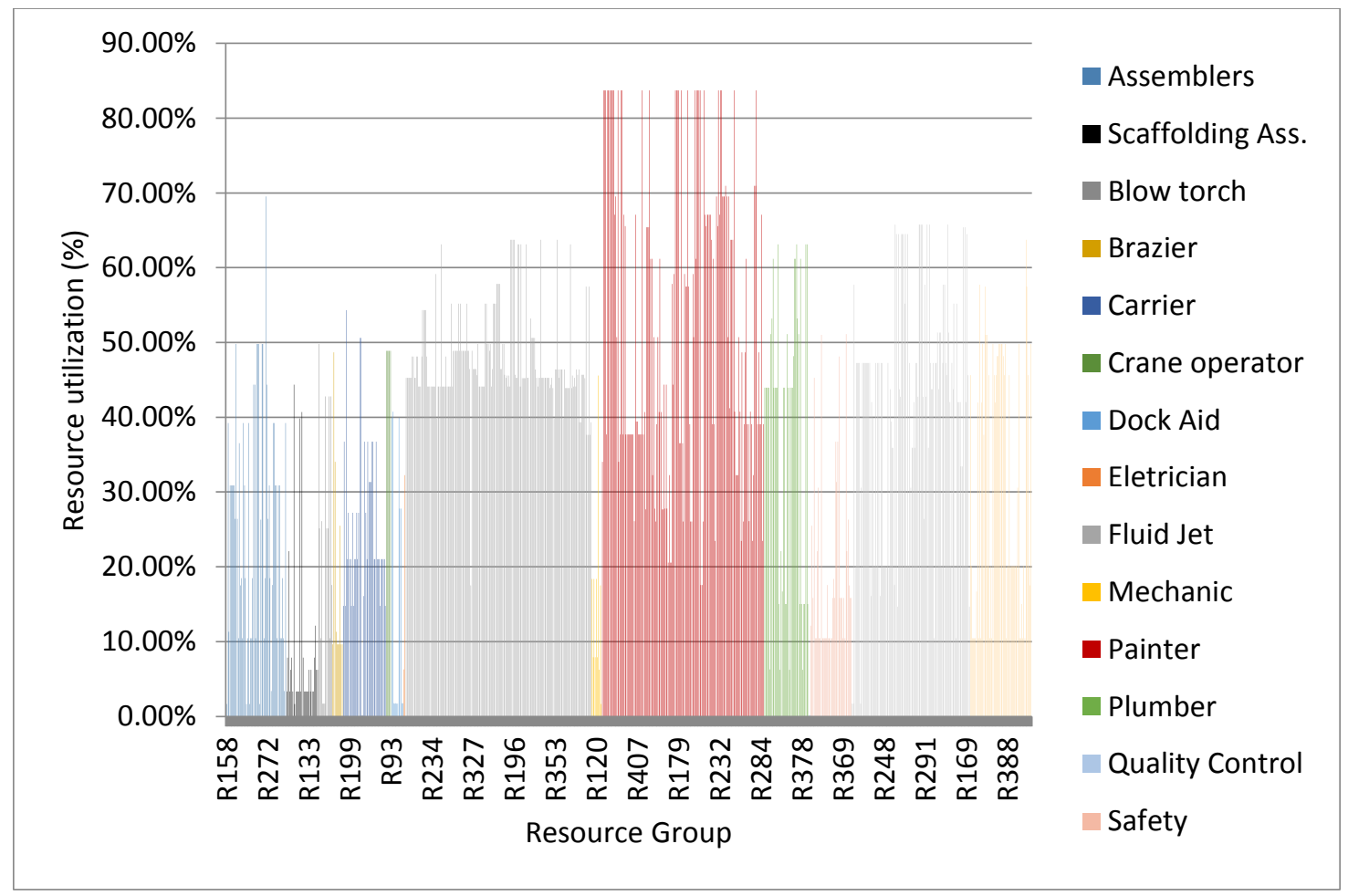

Figure 41: Resource Utilization (Run 1) 
Table AIV.4 shows the individual cost and schedule for all the 111 tasks. Table 48 shows the total project cost and project duration.

\section{Table 48: Project Duration and Cost Analysis (Run 1)}

\begin{tabular}{|c|c|c|c|}
\hline Start date & Finish date & Project Cost (USD) & Project Duration (days) \\
\hline $5 / 18 / 2015$ & $7 / 27 / 2015$ & $\$ 9,876,833$ & 70 \\
\hline
\end{tabular}

Figure 42 shows the accumulated cost among tasks. X-axis represent the tasks, which are numbered from 1 to 111 . $\mathrm{Y}$-axis represents the accumulated cost associated for performing tasks in millions (USD).

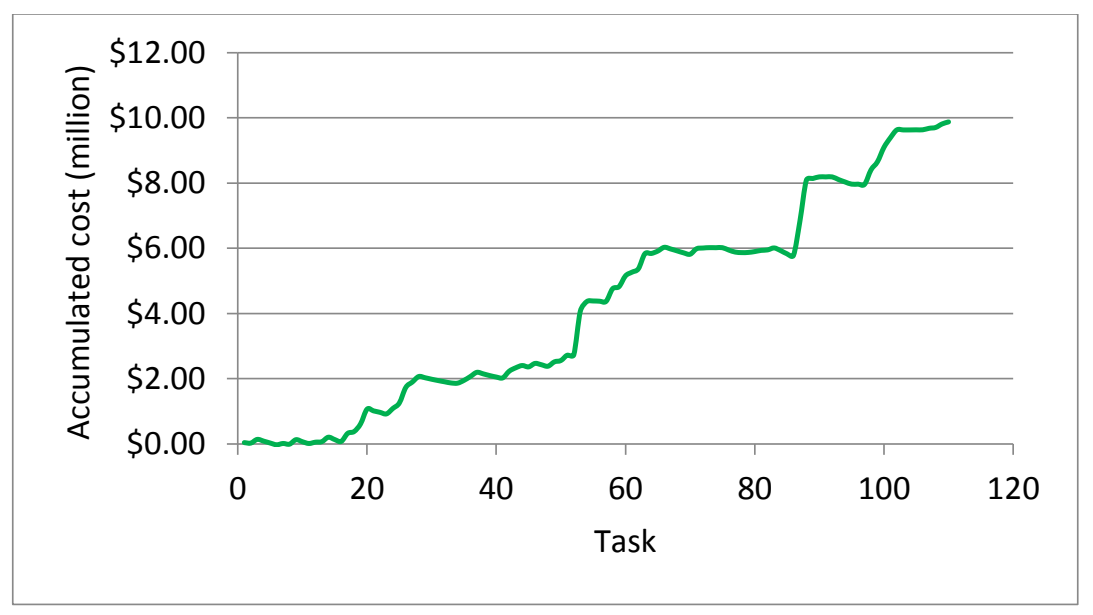

Figure 42: Accumulated Cost with nonrenewable issue in Task 61

Figure 43 shows the deviations against tasks due dates. Task 61 has a positive deviation of 12 days (late) as can be seen in the Figure 43 or by looking at Table AIV.4 in the Appendix IV. Table 47 shows that Task 61 has a waiting time due to a late delivery of the nonrenewable resource (electrode ok 4600.1 / 8). Thus, the deviation shown in the Figure 38 is caused by the nonrenewable resource. What-if the delivery date for the nonrenewable resource could be moved to 03-Jun-2015. How much cost could be saved? Simulation 2 is run to answer this question. Figure 44 shows the Tardiness analysis screen provided by STREAM. 


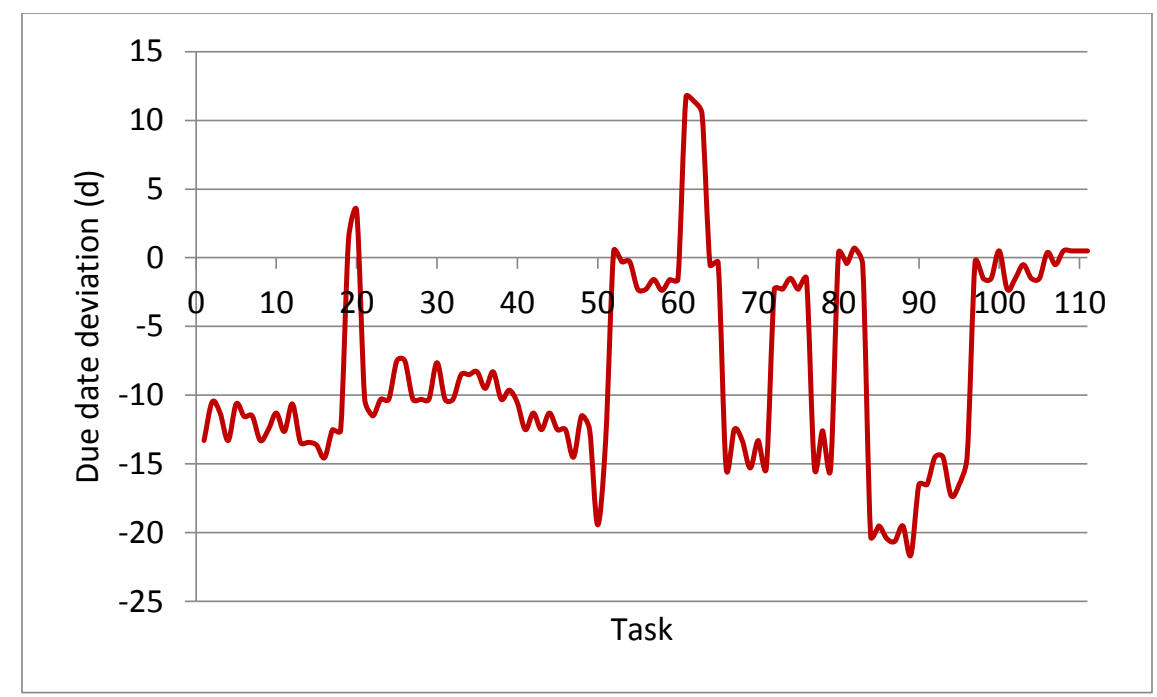

Figure 43: Deviation of Due Dates with nonrenewable resource issue
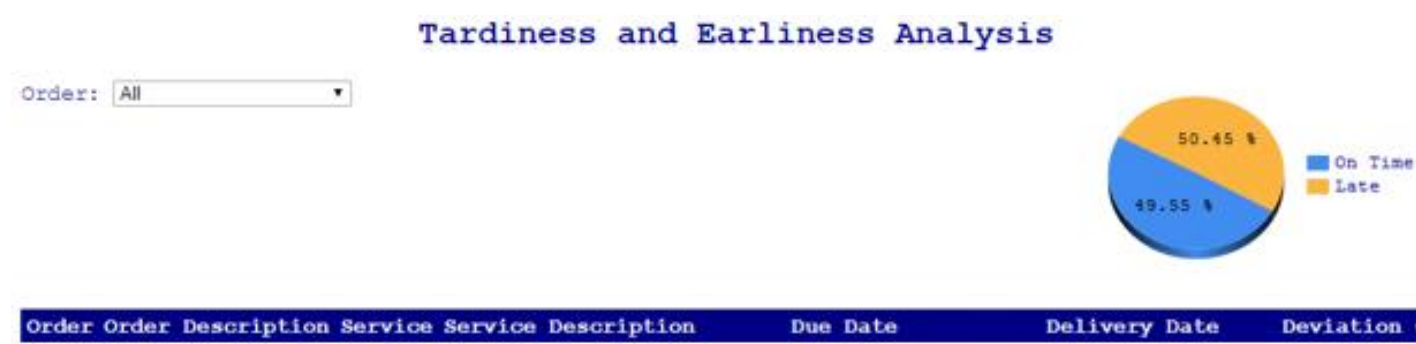

\section{Due Date}

\begin{tabular}{|c|c|c|c|c|c|c|}
\hline 60 & 260 & 60 & 760 & $6 / 5 / 2015 \quad 12: 00: 00 \mathrm{AM}$ & $6 / 15 / 2015 \quad 10: 00: 03 \mathrm{AM}$ & 10.42 \\
\hline 63 & 263 & 63 & 263 & $6 / 28 / 2015 \quad 12: 00: 00$ ANt & $7 / 8 / 2015$ 11:00:00 Avt & 10.46 \\
\hline 64 & 264 & 64 & T64 & $7 / 17 / 2015 \quad 12: 00: 00 \mathrm{AM}$ & $7 / 16 / 2015$ 11:00:02 AM & -0.54 \\
\hline 66 & 766 & 66 & 766 & $6 / 13 / 2015 \quad 12: 00: 00 \mathrm{AN}$ & $6 / 9 / 2015$ 5:00:03 pN & -3.29 \\
\hline 67 & 767 & 67 & T67 & $6 / 22 / 2015 \quad 12: 00: 00 \mathrm{AM}$ & $6 / 19 / 2015: 12: 00: 02 \mathrm{PM}$ & -2.5 \\
\hline
\end{tabular}

Figure 44: Tardiness Analysis screen in STREAM

Figure 45 shows the accumulated cost among tasks for run 2. A reduction in cost for Task 61 could be obtained due to reductions in penalty cost associated for this task. 


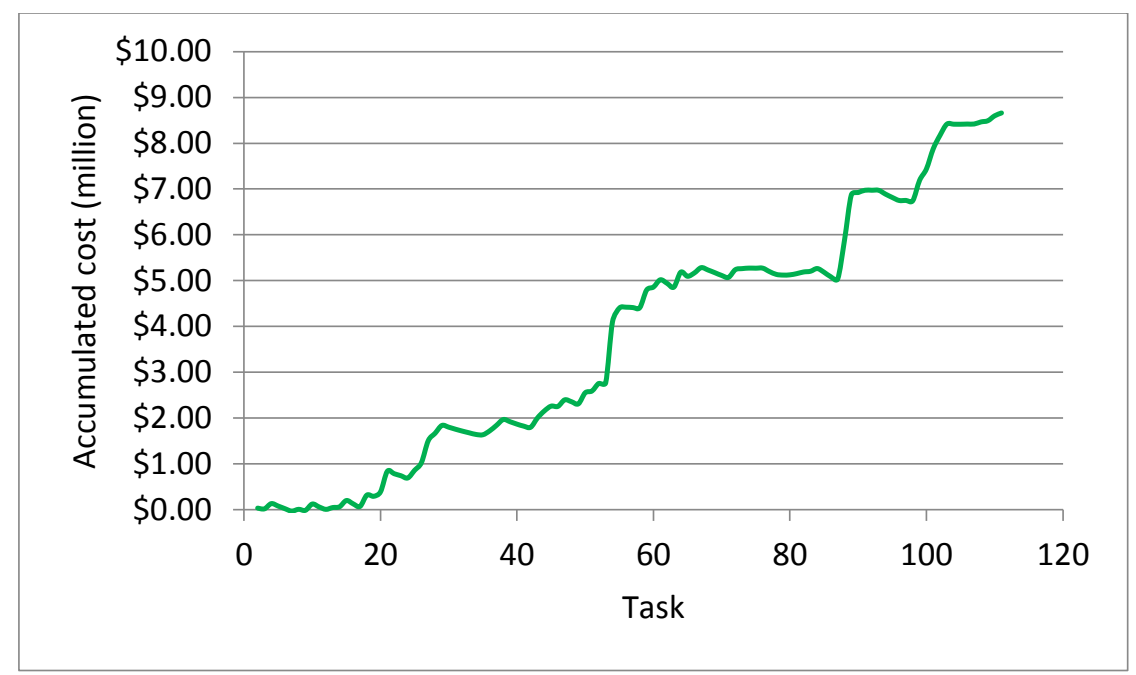

Figure 45: Accumulated cost with earlier deliver date for the nonrenewable resource

Figure 46 shows the deviations against tasks due dates after re-planning the delivery date for the nonrenewable resource (Run 2). Task 61 has no longer a positive deviation of 12 days, instead Task 61 has finished 14 days earlier than its due date. More than $\$ 1,000,000$ in cost could be saved and 5 days in project reduction was found by re-planning the nonrenewable resource. Table 49 shows the project cost and project duration obtained by STREAM for the shipyard.

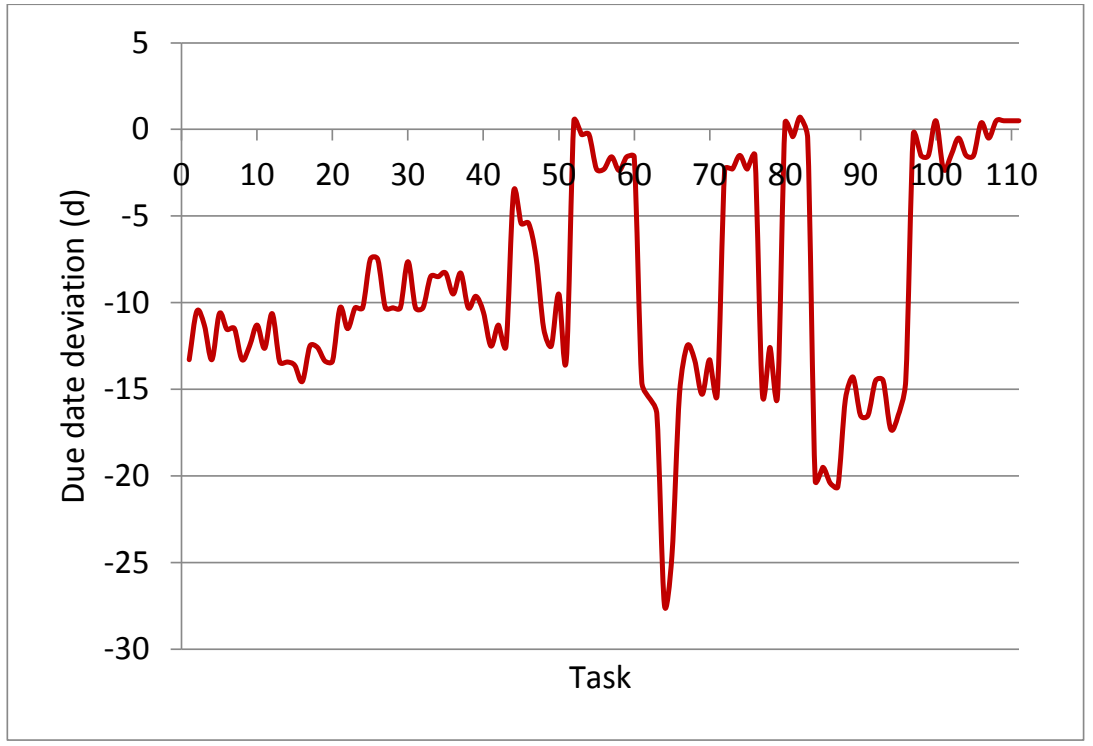

Figure 46: Deviation of Due Dates with Nonrenewable resource issue solved 


\section{Table 49: Project Duration and Cost Analysis}

\begin{tabular}{|c|c|c|c|}
\hline Start date & Finish date & Project Cost (USD) & Project Duration (days) \\
\hline $5 / 18 / 2015$ & $7 / 22 / 2015$ & $\$ 8,660,104$ & 65 \\
\hline
\end{tabular}

Figure 47 shows the Resource schedule screen in STREAM. This screen provides which tasks should be performed during the project for a given resource. The project manager has the option to select one resource and evaluate which tasks will be execute by it. Resource 1 will execute Tasks 75, 76, 77, 68, 69 , and 70 on $6 / 3 / 2015,6 / 10 / 2015,6 / 16 / 2015,6 / 18 / 2015,7 / 1 / 2015$ and $7 / 14 / 2015$, respectively, as can be seen in Figure 47.

\section{Resource Schedule}

\begin{tabular}{|c|c|c|c|c|c|}
\hline Task Id & Due Date & Duration & Eligible Time & start Time & End Time \\
\hline 75 & $5 / 31 / 201512: 00: 00 \mathrm{AM}$ & 1.5 & 6/3/2015 12:00:00 PM & $6 / 3 / 201512: 00: 00 \mathrm{PM}$ & $6 / 9 / 2015$ 5:00:02 PM \\
\hline 76 & 6/6/2015 12:00:00 AM & 1.5 & 6/9/2015 5:00:02 PM & 6/10/2015 7:59:59 AM & $6 / 16 / 201512: 00: 01 \mathrm{PM}$ \\
\hline 77 & $6 / 21 / 2015 \quad 12: 00: 00 \mathrm{AM}$ & 0.5 & $6 / 16 / 201512: 00: 01 \mathrm{PM}$ & 6/16/2015 1:00:01 PM & 6/17/2015 5:00:01 PM \\
\hline 68 & 7/2/2015 12:00:00 AM & 3 & 6/9/2015 5:00:03 PM & 6/18/2015 8:00:00 AM & $6 / 30 / 20155: 00: 03 \mathrm{PM}$ \\
\hline 69 & $7 / 17 / 201512: 00: 00 \mathrm{AM}$ & 3 & 6/30/2015 5:00:03 PM & 7/1/2015 7:59:59 AM & 7/13/2015 5:00:03 PM \\
\hline 70 & $7 / 20 / 2015 \quad 12: 00: 00 \mathrm{AM}$ & 1 & 7/13/2015 5:00:03 PM & 7/14/2015 7:59:59 $\mathrm{AM}$ & 7/16/2015 5:00:01 PM \\
\hline
\end{tabular}

Figure 47: Resource schedule screen in STREAM

\subsection{Case Study 4: Supply Chain Management of Motorcycle Assembly Line}

Supply chain (SC) is as an added value stream to manufacturing goods. It includes various stages of manufacturing, supply of parts, production, supply of raw materials, intermediate parts, packaging, transportation, warehousing, and other logistics [Chen and Hall, 2007]. The Council of Supply Chain Management Professionals [CSCMP, 2014] define Supply Chain Management (SCM) as all tasks involved in sourcing and procurement, conversion, and all logistics management tasks. It also includes coordination and collaboration along stakeholders. SCM is a complex activity due to constant changes and uncertainties in the global market place [Ivanov and Sokolov, 2012]. Mulani and Lee (2002) state 
that supply chain managers spend more than half of their working time dealing with changes in the supply chain.

Reliable delivery dates are critical drivers to sustain a healthy supply chain. Partnership along a supply chain, in which suppliers and customers exchange information (purchase orders, invoices, shipping notices, etc), has worked efficiently to reduce supply issues. However, such healthy relationship is not always possible. Factors such as political instability, financial risks, inflation, tax rates and even cultural issues can affect the stability of a SC.

This case study deals with a highly unstable supply chain where suppliers fail to meet delivery schedule and Just-in-time techniques are not feasible. A framework of supply chain network is presented. The network model is described by the Task-on-Node (ToN) diagram.

Significant amount of research has been conducted in the general area of Supply Chain Scheduling (SCS). Bulk of this research has focused on mathematical formulations, based on job shop and flow shop scheduling methods. In order to deal with the complexity of the scheduling problems, a wide variety of techniques were described in the Chapter 1.

Ivanov and Sokolov (2012) and Ivanov et al (2014) proposed an approach based on optimal control theory, specifically, on Optimal Program Control (OPC), combined with optimization methods for scheduling a supply chain. Figure 48 shows a general supply chain structure based on Ivanov et al (2014).

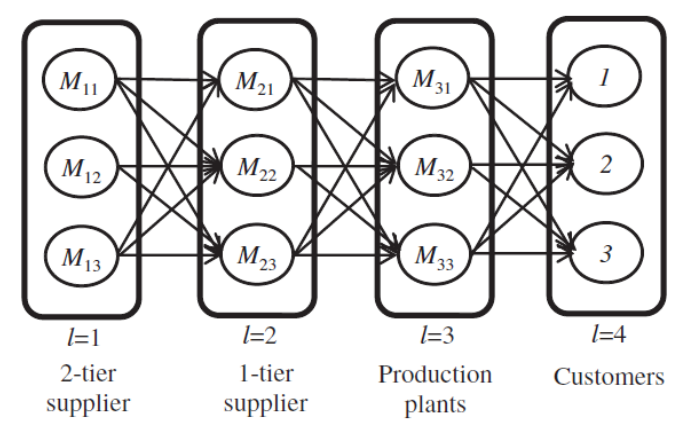

Figure 48: Supply chain structure from Ivanov et al (2014) 
The above supply chain structure consists of 4 processing and 3 transportation stages. $\mathrm{M}_{\mathrm{ij}}$ are the non-identical alternative production plants defined at each stage. Note that stages $1=1,1=2,1=3$ have 3 non-identical alternative resources each and $l=4$ is composed of 3 different customers.

Scheduling of tasks in a project and in a supply chain address several of the same problems. This work applied the algorithms described in Chapter 3. The concept of multi-stage presented earlier, such as 1st -tier, nth-tier suppliers, can be applied to supply chain network. However, in many cases, due to precedence among suppliers and customers along a chain, the notion of stages can become cumbersome. In this study nodes are used to describe precedence relationships. Figure 49 shows a task on node diagram for supply chain network. The supply chain network diagram shows tasks arranged in a logical precedence order along with suppliers and customers. The proposed approach makes the following assumptions:

1. The nodes represent tasks and arrows define the precedence relationship along the chain.

2. Each node represents a supplier or a customer, but a given node can either be a supplier or a customer. For instance in Figure 49, node 2 in the first chain is a supplier of node 4, but it is a customer of node 1. A predecessor node is a supplier to the successor node (customer). For modelling purposes, a supplier is any node with outgoing arrow, which delivers services or goods. A customer is any node with incoming arrows, which is connected with a supplier.

3. Release dates for tasks are unknown in advance. During the simulation cycle, tasks become eligible, subject to precedence. For processing tasks, resource requirements need to be satisfied.

4. A mode is an option to perform a given task, e.g., each mode can be functioning as a facility of a given supplier, a different supplier (backup supplier) or simply a list of different resources to deliver services or goods. Modes might have their resources occupied, delaying the supply chain flow.

Each node can have several modes or several alternative executors as found in Ivanov et al (2014). Instead of having explicitly alternative executors being part of the network (supply chain structure), this work proposes they work as attribute of the node. Only one mode is selected at a particular 
point in time. The customer might have to choose from three different suppliers, however, only one supplier is selected.

In Figure 49, the three rectangular boxes show three simultaneous chains. The number of modes for each node depends upon how many suppliers a customer has. Double circles for Task 1 in chain 1 indicates that some work has already been performed by this supplier. Node ST is the dummy start node for all chains; it is used to guarantee that all eligible suppliers can start producing goods at time 0 . Node EN is the dummy end node; it registers completion time of last node of multiple chains. The meanings of nodes and modes can be different for different situations, however main features of the ToN network are preserved.

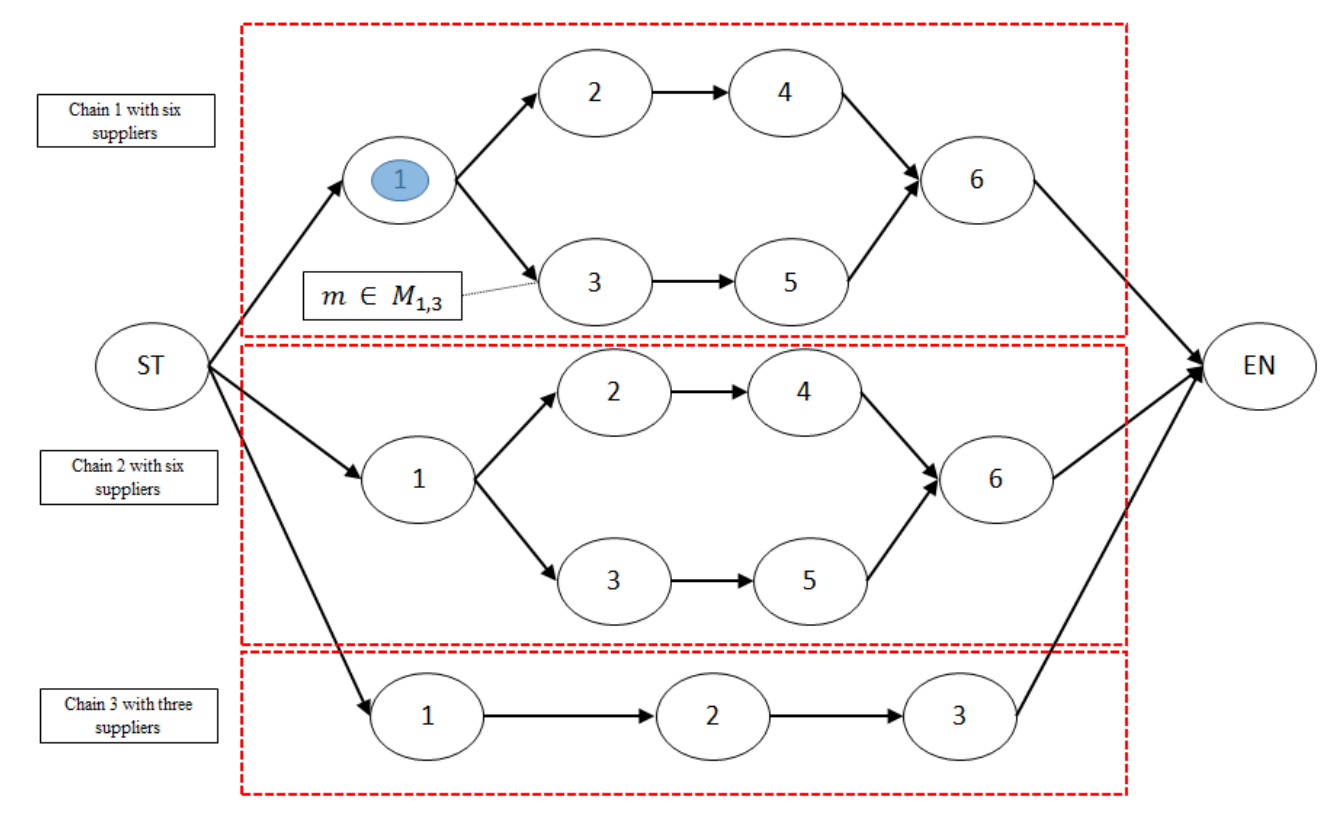

Figure 49: Supply chain schema

\subsubsection{Inputs}

The data for the motorcycle assembly case study comes from a real world company based in Manaus, Brazil. It is the third largest annual motorcycle production facility in the world, only behind facilities located in China and India. It is also the second largest foreign investment of a Japanese company. More than two millions motorcycles are produced each year. It produces more than 20 
different motorcycle types. Once the motorcycles have been assembled, they are shipped to more than 600 dealers in 5 different regions of Brazil.

Several certified suppliers provide different parts (plastic parts, seats, rims, electronic components, tail-pipes, frames, fuel tanks, forks, wheels, covers, engine housings, cylinders, cylinder heads, etc.) to the motorcycle assembly facility and ultimately to assembly lines. The suppliers are under pressure to deliver, at the agreed price, high quality parts, in a timely manner. In order to ensure these requirements, the motorcycle company keeps its own staff at the supplier facilities. However, the issue of late deliveries remains a major cause of concern. There is lack of information on the current status of the supply chain (remaining time to accomplish tasks). Such information if available, could result in rescheduling of tasks. If one supplier fails, it affects the entire supply chain. Shortcomings of the current scenario are: i) lack of information when needed along the chain, ii) lack of coordination in delivering parts to the assembly lines, generating unnecessary inventory, iii) trucks waiting for parts, and iv) dealers not receiving what they were promised.

Figure 50 illustrates the SC network for motorcycle facility. This case study identified 55 tasks, which require 66 resources. Deterministic times were used. The computational time to find a feasible solution was about 2 seconds. The proposed approach schedules the supply chain based on current status of tasks; it enables managers to better handle day-to-day unexpected events.

Tables 50, 51 and 52 show nodes, modes and resources, respectively. In Figure 49, nodes 2 to 14 have 3 modes each (i.e., they have 3 different suppliers to deliver plastic parts, seats, rims, etc). These nodes (suppliers) have no shared resources. For modelling purposes, they are regarded as non-constrained resource. It is assumed the lead times (duration times) are provided by each supplier or calculated by other techniques such as Carvalho et al. (2013).

Nodes 15 to 27 describe shared resources such as box trucks. These are constrained resources. Eight types of trucks support the shipping process to the motorcycle facility. Nodes 28 to 40 describe the 
six workforce teams that unload trucks from different suppliers. These teams are also constrained resources.

Nodes 41, 42 and 43 correspond to preassemble operations, but 2 preassemble teams are allocated. Node 44 represents the assembly operation. Since assembly lines are setup for a particular motorcycle models, this case study considers only one duration time for this node. Nodes 45 and 46 have a duration time and they are not resource constrained.

Nodes 47 to 51 describe three shared workforce teams working on a wharf to load the trucks with the demanded motorcycles. These workforce teams are constrained. Nodes 52 to 56 represent travel time to deliver the motorcycle to different regions in Brazil. Each region has its own fleet of truck which are not resource constrained. Node ST is a start dummy node; it allows suppliers to start their productions simultaneously at given date. Node EN is an end dummy node. It shows that all requested motorcycles have been delivered to the dealers.

This case study assumes a demand of 10,000 units of a given motorcycle type. Based on its Bill of Materials (BOM), different parts are required along the supply chain. Duration times represent the total time to produce individual components, to ship components, to preassemble, and to do final assemble of the 10,000 units. For modelling purposes, the lot size for each supplier refers to assembly of 10,000 units (e.g. for a demand of 10,000 motorcycles, 20,000 wheels are required from the wheels suppliers, if no inventory exist). The lead time (duration) provided by supplier 1 (node 2 or Task 2 ) is 80 hours as shown in Table 50. Each supplier can have its internal lot size, however, this case study assumes each supplier provides the lead time to the motorcycle assembly facility. Due to confidentiality reasons, some of the data values were modified. Table 50 shows some node-mode durations. Tables 51 and 52 describe the nodes (tasks), and resources required for the case study, respectively. 
Table 50: Nodes, modes and duration times from Figure 49

\begin{tabular}{|c|c|l|}
\hline N-M Pair & Hours & Description \\
\hline$(2,1)$ & 80 & Production Plastic Parts \\
\hline$(2,2)$ & 96 & Production Plastic Parts \\
\hline$(3,1)$ & 160 & Production Seats \\
\hline$(4,1)$ & 112 & Production Rims \\
\hline$(5,1)$ & 320 & Production Wheels \\
\hline$(41,1)$ & 160 & Preassemble Rims and Wheels \\
\hline$(44,1)$ & 48 & Assembling motorcycles \\
\hline
\end{tabular}

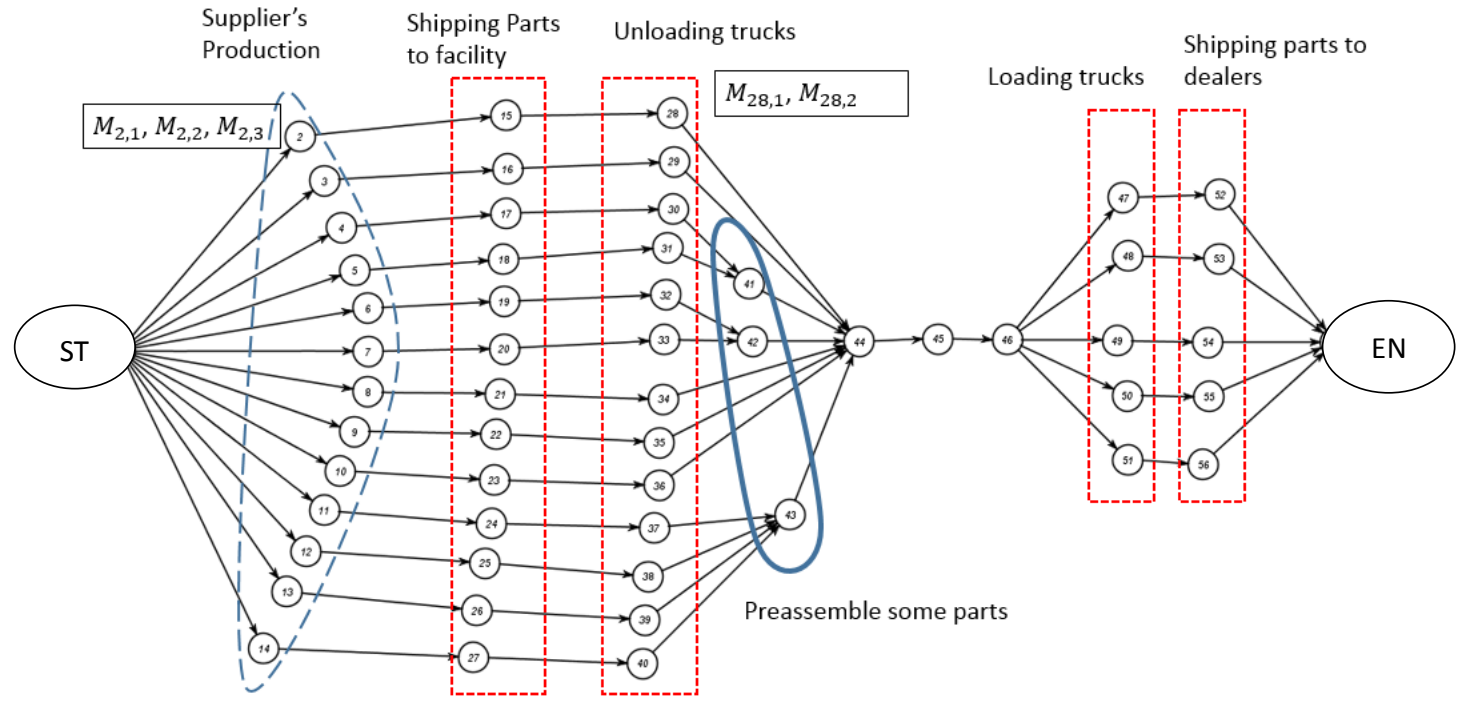

Figure 50: Motorcycle supply chain network 


\begin{tabular}{|c|c|c|c|}
\hline \multicolumn{4}{|c|}{ Table 51: Node description } \\
\hline Node & Description & Node & Description \\
\hline 1 & Start dummy node & 30 & Rims \\
\hline \multicolumn{2}{|c|}{ Production in suppliers } & 31 & Wheels \\
\hline 2 & Plastic Parts & 32 & Electronic components \\
\hline 3 & Seats & 33 & Frames \\
\hline 4 & Rims & 34 & Fuel tanks \\
\hline 5 & Wheels & 35 & Tail pipes \\
\hline 6 & Electronic components & 36 & Forks \\
\hline 7 & Frames & 37 & Covers \\
\hline 8 & Fuel tanks & 38 & Cylinders \\
\hline 9 & Tail pipes & 39 & Cylinders Heads \\
\hline $\mathbf{1 0}$ & Forks & 40 & Engine housings \\
\hline 11 & Covers & \multicolumn{2}{|r|}{ Preassembling parts } \\
\hline 12 & Cylinders & 41 & Rims and Wheels \\
\hline 13 & Cylinders Heads & 42 & Electronic Component and Frame \\
\hline 14 & Engine housings & 43 & Covers, Cylinders, Cylinders Head and Engine Housing \\
\hline \multicolumn{2}{|c|}{ Shipping parts to facility } & \multicolumn{2}{|r|}{ Assembling, shipping, loading trucks } \\
\hline 15 & Plastic Parts & 44 & Assembling motorcycles \\
\hline 16 & Seats & 45 & Shipping by box trucks \\
\hline 17 & Rims & 46 & Shipping by ships \\
\hline 18 & Wheels & 47 & Load trucks to North \\
\hline 19 & Electronic components & 48 & Load trucks to Northeast \\
\hline 20 & Frames & 49 & Load trucks to Central West \\
\hline 21 & Fuel tanks & 50 & Load trucks to South \\
\hline 22 & Tail pipes & 51 & Load trucks to Southeast \\
\hline 23 & Forks & 52 & Shipping to North \\
\hline 24 & Covers & 53 & Shipping to Northeast \\
\hline 25 & Cylinders & 54 & Shipping to Central West \\
\hline 26 & Cylinders Heads & 55 & Shipping to South \\
\hline 27 & Engine housings & 56 & Shipping to Southeast \\
\hline 27 & Engine housings & 57 & End dummy node \\
\hline \multicolumn{4}{|c|}{ Unloading trucks into the facility } \\
\hline 28 & Plastic Parts & & \\
\hline 29 & Seats & & \\
\hline
\end{tabular}




\begin{tabular}{|c|c|c|c|}
\hline \multicolumn{4}{|c|}{ Table 52: Resource description } \\
\hline Resource & Description & Resource & Description \\
\hline R1 & Supplier 1 of Plastic Parts & R34 & Supplier 1 of Cylinders Heads \\
\hline $\mathrm{R} 2$ & Supplier 2 of Plastic Parts & R35 & Supplier 2 of Cylinders Heads \\
\hline R3 & Supplier 3 of Plastic Parts & R36 & Supplier 3 of Cylinders Heads \\
\hline $\mathrm{R} 4$ & Supplier 1 of Seats & R37 & Supplier 1 of Engine housings \\
\hline $\mathrm{R} 5$ & Supplier 2 of Seats & $\mathrm{R} 38$ & Supplier 2 of Engine housings \\
\hline R6 & Supplier 3 of Seats & $\mathrm{R} 39$ & Supplier 3 of Engine housings \\
\hline $\mathrm{R} 7$ & Supplier 1 of Rims & $\mathrm{R} 40$ & Box Truck 1 \\
\hline $\mathrm{R} 8$ & Supplier 2 of Rims & $\mathrm{R} 41$ & Box Truck 2 \\
\hline R9 & Supplier 3 of Rims & $\mathrm{R} 42$ & Box Truck 3 \\
\hline $\mathrm{R} 10$ & Supplier 1 of Wheels & $\mathrm{R} 43$ & Box Truck 4 \\
\hline R11 & Supplier 2 of Wheels & $\mathrm{R} 44$ & Box Truck 5 \\
\hline $\mathrm{R} 12$ & Supplier 3 of Wheels & $\mathrm{R} 45$ & Box Truck 6 \\
\hline R13 & Supplier 1 of Electronic components & R46 & Box Truck 7 \\
\hline R14 & Supplier 2 of Electronic components & $\mathrm{R} 47$ & Box Truck 8 \\
\hline $\mathrm{R} 15$ & Supplier 3 of Electronic components & $\mathrm{R} 48$ & Unloading trucks team 1 \\
\hline R16 & Supplier 1 of Frames & $\mathrm{R} 49$ & Unloading trucks team 2 \\
\hline $\mathrm{R} 17$ & Supplier 2 of Frames & $\mathrm{R} 50$ & Unloading trucks team 3 \\
\hline $\mathrm{R} 18$ & Supplier 3 of Frames & R51 & Unloading trucks team 4 \\
\hline R19 & Supplier 1 of Fuel tanks & R52 & Unloading trucks team 5 \\
\hline $\mathrm{R} 20$ & Supplier 2 of Fuel tanks & R53 & Unloading trucks team 6 \\
\hline $\mathrm{R} 21$ & Supplier 3 of Fuel tanks & R54 & Preassemble team 1 \\
\hline $\mathrm{R} 22$ & Supplier 1 of Tail pipes & R55 & Preassemble team 2 \\
\hline $\mathrm{R} 23$ & Supplier 2 of Tail pipes & R56 & Assemble team \\
\hline $\mathrm{R} 24$ & Supplier 3 of Tail pipes & R57 & Trucks with motorcycle \\
\hline $\mathrm{R} 25$ & Supplier 1 of Forks & R58 & Ships with motorcycle \\
\hline $\mathrm{R} 26$ & Supplier 2 of Forks & R59 & Load trucks with Motorcycle team 1 \\
\hline $\mathrm{R} 27$ & Supplier 3 of Forks & R60 & Load trucks with Motorcycle team 2 \\
\hline $\mathrm{R} 28$ & Supplier 1 of Covers & R61 & Load trucks with Motorcycle team 3 \\
\hline $\mathrm{R} 29$ & Supplier 2 of Covers & R62 & Trucks region North \\
\hline $\mathrm{R} 30$ & Supplier 3 of Covers & R63 & Trucks region Northeast \\
\hline R31 & Supplier 1 of Cylinders & R64 & Trucks region Central west \\
\hline R32 & Supplier 2 of Cylinders & R65 & Trucks region South \\
\hline R33 & Supplier 3 of Cylinders & R66 & Trucks region Southeast \\
\hline
\end{tabular}

Priority rules such as best quality, lowest cost, fastest production, and fastest shipping were used to break a tie when modes required the same resources at the same time. Starting date of 10/9/2014 was selected. All of the motorcycles were delivered on 11/22/2014. Figures 51 shows the Gantt chart provided by STREAM with the scheduling results. Y-axis shows list of tasks which have to be performed while $\mathrm{x}$ axis represents the time. Rectangles shows when tasks are being planned to be performed. 

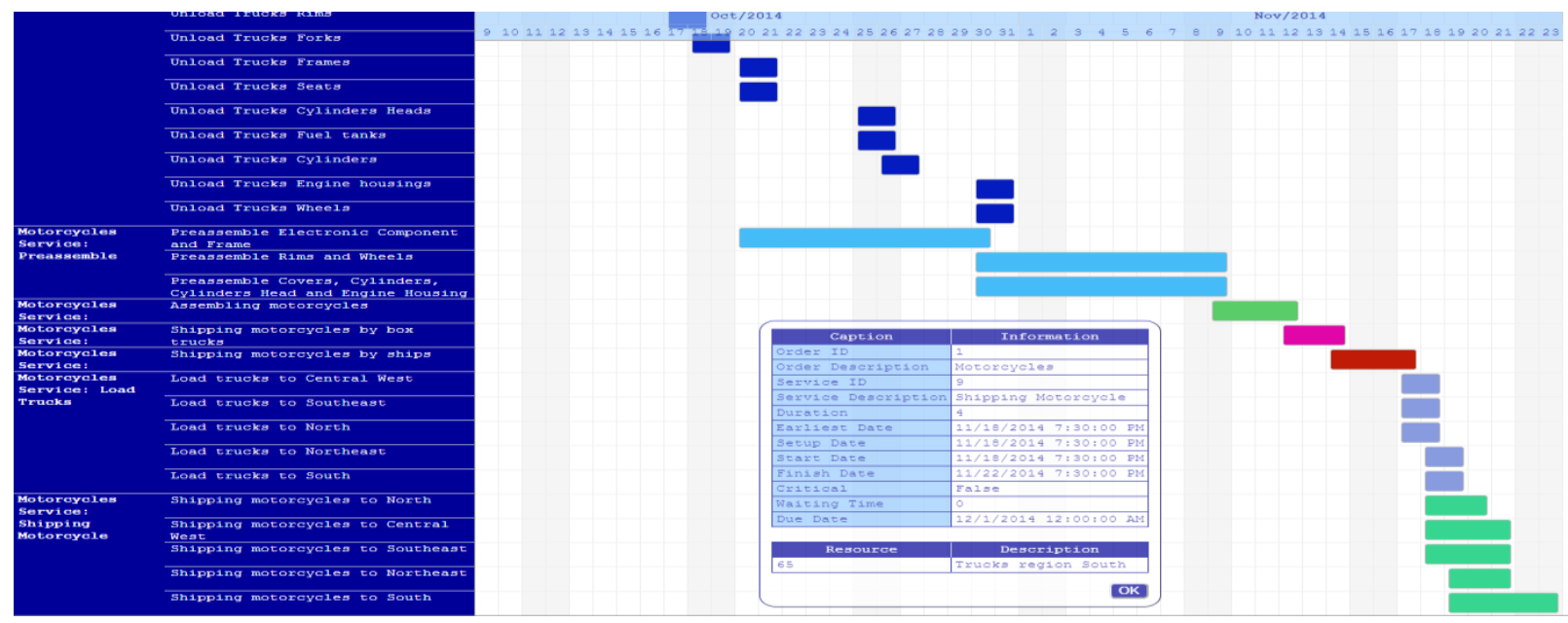

Figure 51: Gantt chart in STREAM for case study 4

The method allows supply chain managers to visualize the current status of the supply chain in a dynamic manner. If the system changes, the supply chain manager can generate the new schedule in near real time. Such approach enhances manager's ability to respond to unexpected events. 


\section{Chapter 5: Conclusions and Contributions}

\subsection{Conclusions}

There are major gaps between project scheduling theory and practice. Many authors [Maenhout and Vanhoucke, 2015], [Abrantes and Figueiredo, 2015], [Coughlan et al., 2015], [Xu and Feng, 2014], [Naber and Kolisch, 2014], [Rehm and Thiede, 2012] have indicated a need to expanded project scheduling methods to meet the needs of "real-world" projects. This research provided one such extension. It expanded the RCPSP methods to deal with multiple task modes and multiple skills and calendars for resources. The revised extension of the RCPSP is called CMRCMPSP. The solution method was implemented into a software tool called STREAM. The object of the method remains the same, that is, minimizing total project duration and cost, subject to resource constraints.

Resource allocation problems are typically formulated mathematically, as an optimization problem, with an objective to minimize total project duration, subject to a set of resource constraints. Such methods attempt to provide a single "optimal" solution. There are many benefits to formulating the program mathematically. However, such formulations do not support large scale project scheduling problems and make several assumption in order to keep the problem mathematically tractable. They assume that resources will remain somewhat stable during project lifecycle. In "real world" projects resources and priorities change of a daily basis. The methodology presented in this research is based on a flexible discrete event simulation, where project manager is an integral part of the resource allocation process. Project manager is provided a method to dynamically adapt to changes in resources and priorities.

The proposed approach was applied to four "real-world" case studies: 1) Construction of a single cell box culvert, 2) Oil refinery turnaround, 3) Ship maintenance, and 4) Motorcycle assembly. First two case studies were based on data provided in the literature. The purpose of these case studies was to validate the results obtained by STREAM with those published in the literature. The ship maintenance and 
motorcycle assembly case studies were based on experience of the author in dealing with complex scheduling issues. These cases demonstrate advanced features (such as, multi-mode tasks, multi-skilled resources, combination of multi-modes and multi-skills, renewable and non-renewable resources) of STREAM that are not addressed in literature.

Regarding the modelling of input data, there is commonly a trade-off between a model's accuracy in capturing the relevant features of the project-planning environment and the resulting model complexity. In this research, different options to perform tasks (multi-modes), multi-skilled workers, and nonrenewable resources, which often exist in real-life projects, were considered in the assessed scenarios to validate STREAM. However, it is also often that during the project scheduling and resource allocation decisions, these realistic options are not taken into account, mainly due to the lack of suitable tools for project planning. The resource allocation decision is left purely to the execution level, where the impact on project duration and cost is difficult to be computed. Ultimately the poor resource management results on cost overruns and schedule slippage.

Another issue discussed herein refers to STREAM's flexibility to represent the decision-maker's perspective towards the planning problem. For instance, project managers are highly knowledgeable professionals and are in the centre of the decision making process. Techniques which oversimplify the project problem and neglect the importance of project managers often do not support the day-to-day reality in projects. STREAM allows the project manager to intervene in the construction of the project schedule with managerial decisions (i.e., changing specific input data) in order to generate a concurrent new analysis. Through a sensitivity analysis, it is possible to test different plans evaluating 'what if?' scenarios and policies in an iterative and interactive planning process comparing the overall cost and project duration. These managerial decisions refer to actions such as varying material availability (changing release dates and due dates), adjusting capacity levels (altering the maximum number of working hours for specific resources, maintenance of equipment), and authorizing overtime for specific workers. 
Besides assisting the project manager in choosing a suitable project schedule, STREAM also enhances the decision-making process by providing more detailed and precise information about the planning problem that is usually not available in the most common used tools for project scheduling. For instance, STREAM automatically assigns the resources capable to execute the tasks among the resource pool and shows which resources are utilized to perform each individual task. To provide this information, task/modes in STREAM can require skills instead of specified resources. Resource skills and their skill levels are also considered in this process. The information relative to the task waiting times contributes in environments where matching multiple resources for tasks is needed. This output highlights the tasks that were delayed due to insufficient number of resources. This information permits a timely assessment of possible shortcomings (e.g., the project manager may need to renegotiate a supply delivery date to avoid resource shortage.).

This research addressed the existing theory-practice gap in the area of project management. The gap with respect to the development of decision support tools for short term resource allocation and management within the project scheduling process was addressed. It aimed to reduce project duration and costs in dynamic environments by empowering project managers to assess different resource allocation flexibilities and to avoid non-renewable resource shortages. According to the results obtained, reductions in cost and project duration were found to be relevant when resource allocation flexibilities are considered.

From an academic perspective, the proposed STREAM approach contributes to literature in the project scheduling research area, as there are few studies that explore resource allocation flexibilities in dynamic environments. This research discusses relevant issues, found in real-life project planning contexts that are not fully considered by other researchers, such as the use of multi-mode tasks, multiskilled resources, the combination of multi-mode tasks and multi-skilled resources, parallel scheduling of multiple tasks, priority rules for dynamic queue, multiple calendars with interruptions and non-renewable resources. 
From the practitioner's standpoint, this research provides a pragmatic view of what can be obtained when project managers are empowered to assess different resource allocation flexibilities within the project scheduling process. Four case studies were used to validate and demonstrate STREAM capabilities in reducing project cost and duration. Several different scenarios, based on a real-life project were considered to validate the proposed STREAM approach, which proved to be useful to assess tradeoffs between the use of these resource allocation flexibilities and the total cost and project duration. Moreover, STREAM enhances the decision making process by providing flexibility to represent the decision-maker's perspective towards the planning problem. It also allows the project manager to intervene in the construction of the project schedule with managerial decisions in order to generate concurrent analysis. Besides assisting the project manager in choosing a suitable project schedule, STREAM also provides more detailed and precise information about the planning problem, usually not available in common used tools for project scheduling, thus permitting a timely assessment of possible shortcomings. The results obtained by STREAM for the case studies are summarized as follows:

Case study 1: A single cell box culvert construction in a drainage project described in the literature. Project details are described in Lu et al. (2008). They identified 33 tasks for this project. STREAM outperformed Particle Swarm Optimization (PSO) for two scenarios described in the literature. It was also able to find the optimal solution in about 1 second using the same data. It is worth noting that these results were obtained without mathematical formulation or attempting to find the optimal. STREAM run other scenarios where human resources have multiple skills, where tasks have multiple modes, and where human resources have multiple skills and tasks have multiple modes. For all the different scenarios, the analysis of cost and project duration is provided. Significant cost and time reductions were found through this method. A comparison between the different scenarios is made with the baseline (optimal solution) and the project duration is reduced from 275 days to 218 days (57 fewer days) and total project cost is reduced from $\$ 14,347,972$ to $\$ 6,460,206$, cost reduction of nearly $50 \%$, amounting to $\$ 7,887,765$. 
Case study 2: An oil refinery turnaround project described in the literature. Project details are described in Siu et al. (2015). They identified 107 tasks for this project and 19 different types of resources. Siu et al. (2015) aims to determine the shortest duration project, the earliest finish time for each task and the minimal quantity of resources. They formulated the problem as optimization problem and use traditional mathematical formulation. STREAM was not only able to find a good solution, but it was able to find the same optimal solution found in Siu et al. (2015), a different optimal solution and two other good solutions. Also through this case study is shown how STREAM can support managers in finding unknown parameters such as priorities for tasks which depend upon current needs. Differently of the traditional mathematical formulation where modelers do not have control or can keep on track what happens during the optimization cycle (Input - (black box) - output), this approach provides output such as i) Task waiting times, ii) Lack of resources when needed, and iii) resource utilization. These outputs, showed through this case study, empower managers in reducing project duration, in finding earliest finish time for tasks and in determining the minimal resource requirements. STREAM run 4 simulations for this case study; the first simulation, a different optimal solution than presented in Siu et al. (2015) was found; the second run, the exactly same optimal solution presented in Siu et al. (2015) was obtained; the third simulation, the maximal reduction in number of resources which does not affect the shortest project duration is obtained; the four simulation provides the least number of resource to be added in order to avoid any waiting time for all tasks.

Case study 3: Real world data was collected from the largest shipyard in the southern hemisphere. The shipyard of this case study is located at Rio de Janeiro/Brazil. A project for repairing a complex vessel is described. It is a class leading ROV (remotely operated underwater vehicle) construction support vessel ideally suited to perform subsea operations across a wide range of water depths and environmental conditions. The project was planned with 111 tasks with 475 resources in the resource pool. Steel work, treatment and painting, valves, propeller, tank cleaning, support, and pipes are examples of group of tasks which were scheduled by STREAM. STREAM was able to finish the project in 70 days with a cost of 
$\$ 9,542,465$. A shortage of a nonrenewable resource (electrode ok 4600.1/ 8) delayed task T61 in 26 days. STREAM was able to identify it, and a renegotiation of delivery date was made. The project duration and cost were reduced to 65 days and $\$ 9,058,573$ respectively. STREAM identified the resource shortage and more than $\$ 400,000$ in savings could be obtained. In order to demonstrated the STREAM capability to run in multiple-projects and huge large project problems. STREAM runs a multi-project scenario with 100 projects in parallel similar as Skandi Victoria. The computational time to schedule the 100 projects was 13.5 minutes.

Case study 4: This case study deals with a supply chain scheduling of a motorcycle assembly facility located in Manaus, Brazil. The supply chain tasks were formulated as a network of 55 tasks competing for 66 resources. The method was able to provide a set of feasible solutions in about 1 second of computing time. Priority rules such as best quality, lowest cost, fastest production, and fastest shipping were used to break a tie when modes required the same resources at the same time.

The proposed approach is general purpose and applicable to a wide variety of situations. The proposed research used data from four case studies in different context. The correctness of the method is guaranteed because STREAM was able to find optimal solutions when it run in equal conditions to those case studies which attempted to optimize the problem. It is worth noting that STREAM does not have a mathematical formulation. However, this research showed that STREAM has the potential and delivered optimal solutions in about one second. Also a set of feasible solutions with cost and time trade-offs are always provided. While evolutionary methods and strong mathematical formulations start to have prohibitive computational times and not able to solve large problems, STREAM can solve problems with 3,000 tasks (5,000 modes) and 400 multi-skilled resources in 78 seconds, and with 10,434 tasks (15,000 modes) and 400 multi-skilled resources in 810 seconds. The problem with 10,434 tasks is based on a scenario with 100 projects running simultaneously. Based on the results and computational times obtained by STREAM for scheduling more than 10,000 tasks in realistic scenarios, this approach can be applied to real-life dayto-day projects without prohibitive computational times. 


\subsection{Contributions}

This research enhances decision making capability of project managers by providing a flexible method for resource allocation, in order to reduce total project duration and cost. Specific contributions are described below:

The Resource Constrained Project Scheduling Problem (RCPSP) was expanded to include combinatorial number of task modes, multiple projects, resource skills, and calendars. The proposed RCPSP extension, Combinatorial Multi-mode Resource Constrained Multi-project Scheduling Problem (CMRCMPSP), includes capabilities provided by RCPSP, MRCPSP, RCMPSP, and MRCMPS methods. CMRCMPSP allows for real-life constraints and it makes fewer assumptions than the previous methods. It was shown that a given task with two modes instead only providing two options for executing a task, this research provided innumerous sets. The mode formed by a set of subsets of resources provides higher flexibility to resource allocation compared with previous approaches.

A discrete event simulation has been developed for the CMRCMPSP. It focused on manufacturing, constructions, maintenance of equipment and other systems driven by projects with resource constraints. It was implemented in Visual Basic.Net on a Windows platform and called as STREAM. It does not use the traditional queue concept, instead it selects modes from the eligible modes pool based on resource availability, skill level, and current priority. The Parallel Mode Schedule Generation Scheme is proposed. This is an extension of the Parallel Schedule Generation Scheme. It was shown that traditional queue management cannot support the CMRCMPSP. STREAM was tested and validated on two case studies published in the literature. The two case studies in the literature mathematically formulated the resource allocation problem as an optimization problem. STREAM produced the same results (under the same assumption) as those reported in the literature. After validating the software it was applied to two more case studies.

Focus of most resource allocation methods is on the mid (1 to 3 months) to long ( 3 months to 3 years) term time horizon. Focus of STREAM is on short term planning, that is, on a daily or hourly basis. Short 
term scheduling is significantly more complex than long term scheduling. Long term scheduling systems assume a stable system. STREAM considers the system to be dynamic. It can adapt to real world situations, e.g. a worker could get sick in the afternoon resulting in a changed resource profile. STREAM generates a series of feasible solutions, along with the impact of each solution on schedule and cost. Focus of STREAM is on providing a series of feasible solutions and let the project manager determine what is good under the current circumstances based on measure of performances.

STREAM is better equipped to meet real world needs because it is able to handle 1) Multiple combinatorial modes for tasks, 2) Multiple skills and capabilities for resources, 3) Different priority rules for different resources, and 4) Multiple calendars with interruptions. The two dominant software tools, Microsoft Project and Primavera do not provide such capabilities.

This research produced 3 peer reviewed conference papers [1-Pinha et al., 2015], [2-Pinha et al., 20115], [Pinha and Ahluwalia, 2013] and 3 papers in peer reviewed journals [Pinha and Ahluwalia, 2014], [Pinha et al., 2015], and [Pinha and Ahluwalia, 2015]. The last two are still under review.

\subsection{Future Research}

This research could be expanded to include:

1. A capability to track and report on task status and resource utilization in near real-time: To facilitate the integration of STREAM into the project day-to-day environment, the procedures, software tools and hardware to use STREAM properly must be identified. Questions to be addressed: i) How to collect the task and resource status in an efficient manner to re-plan a project? ii) What time during the day is the best time for project managers to schedule their projects? and iii) how resources will execute the tasks which STREAM has planned?

2. Comparison of proposed schedule with the actual and use of Earned Value Management (EVM) to track project progress: The Earned Value Management theory (EVM) widely used to control project progress could be developed into STREAM. STREAM is able to provide the baseline 
schedule while EVM controls the project progress. The ultimately goal is to verify if STREAM reduces the discrepancy between of what was planned and what was actually performed.

3. Comparison of forward and backward scheduling on project duration and cost: Project duration and costs comparison between forward scheduling and backward scheduling methods could be made in order to identify whether or not reductions can be found.

4. Development of industry based heuristics for priority rules: if STREAM is to be integrated with a number of different project applications which have their own methodologies and rules. The process to facilitate the integration and to identify the rules is subject for further investigation. Standards for the exchange and sharing of the project rules based on the application could be defined.

5. Implementation of STREAM on multiple platforms such as windows, web, mobile devices: Further development is needed to use full capability of STREAM on multiple platform such as windows, web and mobile devices.

6. Application of STREAM to other industries, e.g. energy, healthcare, aerospace, software development: The results obtained so far encourage an investigation whether STREAM fits other industries such as energy, healthcare, aerospace, software development particularly in situations where resource allocation flexibilities exist.. 


\section{References}

Abrantes, R., Figueiredo, J., (2015), International Journal of Project Management, "Resource management process framework for dynamic NPD portfolios", Volume 33, Issue 6, August 2015, Pages 1274-1288, ISSN 0263-7863

Alcaraz, J., Maroto, C., Ruiz, R., (2003), Journal of the Operational Research Society, "Solving the multimode resource-constrained project scheduling problem with genetic algorithms", 54, 614-626.

Araúzo, J., Pajares, J., Lopez-Paredes, A., (2010), Simulation Modelling Practice and Theory, "Simulating the dynamic scheduling of project portfolios", Volume 18, Issue 10, November 2010, Pages 1428-1441, ISSN 1569-190X.

Ballard, G (2000), Ph.D. Thesis, "The Last PlannerTM System of Production Control", School of Civil Engineering, The University of Birmingham.

Baumann, P., Trautmann, N., (2013), In Proceedings of the IEEE international conference on industrial engineering and engineering management, "Optimal scheduling of work-content constrained projects", 10-13 December. Bangkok, Thailand.

Benichou, J. M. Gauthier, P. Girodet, G. Hentges, G. Ribiere, O. Vincent, (1971), Mathematical Programming, "Experiments in mixed-integer linear programming", 1971, Volume 1, Issue 1, pp 76-94,

Beşikci,U., Bilge,U., Ulusoy,G. (2015), European Journal of Operational Research, "Multi-mode resource constrained multi-project scheduling and resource portfolio problem", Volume 240, Issue 1, 1 January 2015, Pages 22-31, ISSN 0377-2217

Bianco, L., Caramia, M., (2013), Flexible Services and Manufacturing Journal, "A new formulation for the project scheduling problem under limited resources", 25, 6-24.

Bouleimen, K. , Lecocq, H., (2003), European Journal of Operational Research, "A new efficient simulated annealing algorithm for the resource-constrained project scheduling problem and its multiple mode version", 149 (2), 268-281.

Browning, T., Yassine, A., (2010), International Journal of Production Economics, "Resource-constrained multi-project scheduling: Priority rule performance revisited", Volume 126, Issue 2, August.

Brucker, P., Knust, S., (2001), Lecture Notes in Computer Science 2079, "Resource-constrained project scheduling and timetabling", 277-293.

Brucker, P., Drexl, A., Mohring, R., Neumann, K., Pesch, E., (1999), European Journal of Operational Research, "Resource-constrained project scheduling: notation, classification, models, and methods", $112: 3-41$

Calhoun, K., Deckro, R., Moore, J., Chrissis, J., Hove, J., (2002), The international Journal of Management Science, "Planning and re-planning in project and production scheduling," 30 (3) 155-170.

Chabane, H., (2004), xa.yimg.com/kq/groups/24634788/1393872093/name/Art111.pdf

Charris, E., Carlos D. Paternina-Arboleda, (2013), International Journal of Industrial and Systems Engineering, "Simulation model of the supply chain on a naval shipyard", Volume 13, Issue - 3, 280290, 2013-01-01, 10.1504/IJISE.2013.052277

Chen, P., Shahandashti, S., (2009), Automation in Construction, "Hybrid of genetic algorithm and simulated annealing for multiple project scheduling with multiple resource constraints", Volume 18, Issue 4, July 2009, Pages 434-443, ISSN 0926-5805. 
Cheng, L.Z.; Hall, G.N. (2008), Manufacturing \& Service Operations Management. "Maximum Profit Scheduling", vol.10, No. 1, winter 2008, pp.84-107.

Choo, H. (2003), Ph.D. Thesis, "Distributed Planning and Coordination to Support Lean Construction", School of Civil Engineering, The University of California, Berkeley.

Chryssolouris, G., J. Pierce, K. Dicke, (1992), International Journal of Flexible Manufacturing Systems, "A decision-making approach to the operation of flexible manufacturing systems", 4 (3-4) (1992) 309330 .

Chryssolouris, G., J. Pierce, K. Dicke, (1991), Journal of Manufacturing Systems, "An approach for allocating manufacturingresources to production tasks", 10 (5) (1991)368-382.

Chryssolouris, G., K. Dicke, M. Lee, (1992), International Journal of Production Research, "On the resources allocation problem", 30 (12) (1992) 2773-2795.

Chryssolouris, G., M. Lee, K. Dicke, (1991), International Journal of Computer Integrated Manufacturing, "An approach to short interval scheduling for discrete parts manufacturing", 4 (3) (1991) $157-168$.

Chryssolouris, G., (2005), Manufacturing Systems: Theory and Practice, 2nd Edition New York, Springer-Verlag, 2005.

Chryssolouris, G., (1999), 10th International Conference on Computer Applications in Shipbuilding, "A Planning and Control Method for Shipyard Processes: A Ship-repair Yard Case Study”, ICCAS 99.

Chryssolouris, G., Makris, S., Xanthakis, V., \&Mourtzis, D., (2004). Journal of Computer Integrated Manufacturing, "Towards the Internet-based supply chain management for the ship repair industry", International 17(1), 45-57. doi:10.1080/0951192031000080885

Costa, R.S. e Jardim E.G.M. (2010), "Os cinco passos do pensamento enxuto", Rio de Janeiro, 2010. http://www.trilhaprojetos.com.br

Cury, 2001, http://www.das.ufsc.br/ cury/cursos/apostila.pdf

De Boer, R. (1998), Ph. D. thesis, "Resource-Constrained Multi-Project Management - A Hierarchical Decision Support System", University Of Twente, The Netherlands.

De Boer, R., J.M.J. Schutten, W.H.M. Zijm, (1997), CIRP Annals - Manufacturing Technology, “A Decision Support System for Ship Maintenance Capacity Planning", Vol. 46, Issue 1, pp. 391-396, ISSN 0007-8506, http://dx.doi.org/10.1016/S0007-8506(07)60850-6 (http://www.sciencedirect.com /science/article/ pii/S0007850607608506)

De Reyck, B., Herroelen, W., (1999), European Journal of Operational Research, "The multi-mode resource-constrained project scheduling problem with generalized precedence relations," 119 (2), 538556.

Dlugokecki, V., Fanguy, D., Hepinstall, L., Tilstorm, D., (2010), Journal of Ship Production and Design, "Transforming the Ship Building and Ship Repair Project Environment", vol. 26, num. 4, pp 265-272, doi: 10.1109/IEEM.2011.6117887

Dlugokecki, V., Fanguy, D., Hepinstall, L., Tilstorm, D., (2010), Journal of Ship Production and Design, "Transforming the Ship Building and Ship Repair Project Environment", vol. 26, num. 4, pp 265-272

DoN, (2013), http://www.onr.navy.mil/ /media/Files/Funding-Announcements/BAA/2013/13-020.ashx

Drexl, A., Nissen, R., Patterson, J., Salewski, F., (2000), European Journal of Operational Research, "Progen/px - An instance generator for resource-constrained project scheduling problems with partially renewable resources and further extensions", 125 (1), 59-72. 
Coughlan, E., Lübbecke, M., Schulz, J., European Journal of Operational Research, “A branch-price-andcut algorithm for multi-mode resource leveling", Volume 245, Issue 1, 16 August 2015, Pages 70-80, ISSN 0377-2217.

Elmaghraby, S., (1977), “Task networks: Project planning and control by network models”, Wiley, New York.

Fazar, W., (1959), The American Statistician, "Program Evaluation and Review Technique", Vol. 13, No. 2, p.10.

Feng, W. [1], Zheng, L., Jingshan, L., (2012). Robotics and Automation (ICRA), "The robustness of scheduling policies in multi-product manufacturing systems with sequence-dependent setup times and finite buffers", 2012 IEEE International Conference on, pp.5074-5079.

Feng, W.[2], Zheng, L., Jingshan, L. (2012), International Journal of Production Research, "Scheduling policies in multi-product manufacturing systems with sequence-dependent setup times and finite buffers", 50:24, 7479-7492.

Framinan, J., and R. Ruiz., (2012), International Journal of Production Research, "Guidelines for the Deployment and Implementation of Manufacturing Scheduling Systems", 50 (7): 1799-1812.

Fundeling, C., (2006), Deutscher Universitat-Verlag, "Resourcen beschränkte Projektplanung bei vorgegebenen Arbeitsvolumina (Resource-constrained project scheduling with work contents).”

Fundeling, C., Trautmann, N., (2010), European Journal of Operational Research, “A priority-rule method for project scheduling with work-content constraints", 203, 568-574.

Hartmann, S., (2001), Annals of Operations Research, "Project scheduling with multiple modes: A genetic algorithm", 102, 111-135.

Hartmann, S., Briskorn, D., (2010), European Journal of Operational Research, "A survey of variants and extensions of the resource-constrained project scheduling problem", Volume 207, Issue 1, 16 November 2010, Pages 1-14, ISSN 0377-2217.

Harvard Review Business, (2003). https://hbr.org/2003/09/why-good-projects-fail-anyway

Hazewinkel, M., (2001), "Graph theory", Encyclopedia of Mathematics, Springer, ISBN 978-1-55608010-4

Heilmann, R., (2001), OR Spektrum, "Resource-constrained project scheduling: A heuristic for the multimode case", 23, 335-357.

Heilmann, R., (2003), European Journal of Operational Research, "A branch-and-bound procedure for the multi-mode resource constrained project scheduling problem with minimum and maximum time lags", 144, 348-365.

Jarboui, B., Damak, N., Siarry, P. , Rebai, A., (2008), Applied Mathematics and Computation, "A combinatorial particle swarm optimization for solving multi-mode resource-constrained project scheduling problems", 195, 299-308.

Jozefowska, J., Mika, M., Rozycki, R., Waligora, G., Weglarz, J., (2001), Annals of Operations Research "Simulated annealing for multi-mode resource-constrained project scheduling," 102, 137-155.

Kelton, W.D., Sadowski, R.P., Sadowski, D.A., 2002, Simulation with ARENA.

kendrickoil (2015, http://www.kendrickoil.com/

Khoshnevis, B., (1994). Discrete systems simulation. New York: McGraw-Hill. 
Kolisch, R., (1996), European Journal of Operational Research, "Serial and parallel resource-constrained project scheduling methods revisited: theory and computation", 90, 320-333.

Kolisch, R., Drexl, A., (1997), IIE Transactions, "Local for multi-mode resource-constrained project", vol. 29, no. 11, pp. 987-999.

Kolisch,R., Meyer,K., Mohr, R., Schwindt, C., Urmann, M., (2003), Zeitschrift für Betriebswirtschaft, Ablaufplanung fur die Leitstrukturoptimierung in der Pharmaforschung (Scheduling of lead optimization in pharmaceutical research projects). $73,825-848$.

Laslo, Z., Goldberg, A., (2008), International Journal of Project Management, "Resource allocation under uncertainty in a multi-project matrix environment: Is organizational conflict inevitable?", Volume 26, Issue 8, November 2008, Pages 773-788, ISSN 0263-7863

Leadership, (2013), http://ec.europa.eu/enterprise/sectors/maritime/files/shipbuilding/leadership2020-finalreport_en.pdf

Law, A. M., and Kelton, W. D. (2000). Simulation modeling and analysis, 3rd Ed., McGraw-Hill, New York.

Lau, S., Lu, M., and Poon, C. (2014), J. Constr. Eng. Manage, "Formalized Approach to Discretize a Continuous Plant in Construction Simulations", 140(8), 04014032.

Lu, M. Lam, H., Dai, F., (2008), Automation in Construction, Resource-constrained critical path analysis based on discrete event simulation and particle swarm optimization, Volume 17, Issue 6, August 2008, Pages 670-681

Lu, M., Chan, W.H., (2004), Proceedings of Winter Simulation Conference 2004, "Modeling concurrent operational delays in construction tasks with simplified discrete event simulation approach", Washington D.C., 2004, pp. 1260-1267

Lu, M., (2003), Journal of Construction Engineering and Management, "Simplified Discrete-Event Simulation Approach for Construction Simulation”, 2003 129:5, 537-546

Maenhout, B., Vanhoucke, M., (2015), Journal of Scheduling, "An exact algorithm for an integrated project staffing problem with a homogeneous workforce", p-1-27, August-2015.

Matloff, N., (2008), http://heather.cs.ucdavis.edu/ matloff/156/PLN/DESimIntro.pdf

MARAD,(2013), http://www.marad.dot.gov/documents/MARAD_Econ_Study_Final_Report_2013.pdf

Mello, M., J. O. Strandhagen, (2011), Proceedings of the Institution of Mechanical Engineers, Part M: Journal of Engineering for the Maritime Environment, "Supply chain management in the shipbuilding industry: challenges and perspectives", 225:261-270, June 24, 2011

MIT Nonlinear (2015). http://web.mit.edu/15.053/www/AMP-Chapter-13.pdf

Moder, J., and Phillips, C., (1964), Project Management with CPM and PERT"

Moghaddam, K., John S. Usher, (2011), Computers \& Industrial Engineering, "Preventive maintenance and replacement scheduling for repairable and maintainable systems using dynamic programming", Volume 60, Issue 4, May 2011, Pages 654-665, ISSN 0360-8352, http://dx.doi.org/10.1016/j.cie.2010.12.021.(http://www.sciencedirect.com/science/article/pii/S036083521 100009X)

Mourtzis, D., (2005), International Journal of Computer Integrated Manufacturing, “An integrated system for managing ship repair operations", 18:8, 721-733

MS Project (2015), http://office.microsoft.com/en-us/project/ 
Naber, A., Kolisch, R., (2014), European Journal of Operational Research, "MIP models for resourceconstrained project scheduling with flexible resource profiles", , Volume 239, Issue 2, 1 December 2014, Pages 335-348, ISSN 0377-2217.

Navarro, P., Iborra, Andrés; Fernández, Carlos; Sánchez, Pedro; Suardíaz, Juan, (2010), Sensors, "A Sensor System for Detection of Hull Surface Defects”, no. 8: 7067-7081.

Navarro, P., Muro, Juan S., Alcover, Pedro M., Fernández-Isla, Carlos, (2013), Sensors, "Sensors Systems for the Automation of Operations in the Ship Repair Industry”, no. 9: 12345-12374.

NSRP, (2013), http://www.nsrp.org/2-Solicitation_Documents/RA\%2012-01_FINAL-v2.pdf

Oracle, (2015), https://www.oracle.com/database/index.html

Ozdamar, L., (1999), IEEE Transactions on Systems, Man, and Cybernetics, Applications and Reviews, "A genetic algorithm approach to a general category project scheduling problem", 29 (1999) 44-59.

Papakostas, N., Papachatzakis, P., Xanthakis, V., Mourtzis, D., Chryssolouris, G. (2010), Decision Support Systems. "An approach to operational aircraft maintenance planning", Volume 48, Issue 4, March 2010, Pages 604-612, ISSN 0167-9236, http://dx.doi.org/10.1016/j.dss.2009.11.010.

Peteghem, V., Vanhoucke, M., (2010), European Journal of Operational Research, "A genetic algorithm for the preemptive and non-preemptive multi-mode resource-constrained project scheduling problem", vol. 201, no. 2, pp. 409-418.

Pinha, D., Ahluwalia, R., Carvalho, A., (2015), Project Management Journal, "Flexible Resource Management and Its Effect on Project Cost and Duration", (Under review).

Pinha, D., and Ahluwalia, R., (2015), European Journal of Operational Research, "Combinatorial MultiMode Resource Constrained Multi-Project Scheduling”, (Under review).

Pinha, D., Ahluwalia, R., Carvalho, A., (2015), 2015 IFAC Symposium on Information Control in Manufacturing, "Parallel Mode Schedule Generation Scheme", Ottawa, Canada.

Pinha, D., Ahluwalia, R., Carvalho, A., Senna, P., (2015), 2015 IFAC Symposium on Information Control in Manufacturing", "Supply Chain Scheduling: A Motorcycle Assembly Case Study", Ottawa, Canada.

Pinha, D., Ahluwalia, R, (2013), Proceedings of the Industrial and System Engineering World Conference, "Decision Support System for Repair Shipyard Industry", Las Vegas, USA.

Pinha, D, Ahluwalia, R, (2014) "Decision Support System for Production Planning in the Ship Repair Industry". Industrial and Systems Engineering Review Journal, [S.1.], v. 2, n. 1, p. 52-61, jul. 2014. ISSN 2329-0188

Pinha, D., De Queiroz, M.H., Cury, J. E R, (2011), Proceedings of the IEEE Conference on Automation Science and Engineering (CASE), "Optimal scheduling of a repair shipyard based on Supervisory Control Theory”, 2011, vol., no., pp.39,44, August 24-27, 2011 doi: 10.1109/n CASE.2011.6042515

PMI, (2013), A Guide to the Project Management Body of Knowledge, Fifth Edition, Project Management Institute.

Primavera, (2015), https://www.oracle.com/applications/primavera/index.html

Pritsker, A. A. B., and O'Reilly, J. J. (1999). Simulation with visual SLAM and AweSim, 2nd Ed., Systems Publishing Corp., West Lafayette,IN

Pritsker, A., Watters, L., Wolfe, P., (1969), Manage. Sci., "Multi project scheduling with limited resources: A zero-one programming approach”, 16, 93-108

ProChain, (2015), https://www.prochain.com/ 
ProTrack, (2015), http://www.or-as.be/protrack

Ranjbar, M., Kianfar, F., (2010), Transaction E: Industrial Engineering, "Resource-constrained project scheduling problem with flexible work profiles: A genetic algorithm approach", 17, 25-35.

Rehm, M., \& Thiede, J., (2012), Technische Universität Dresden, "A survey of recent methods for solving project scheduling problems", Fakultät Wirtschaftswissenschaften.

Sabzehparvar, M., Seyed-Hosseini, S., (2008), Journal of Supercomputing, "A mathematical model for the multimode resource-constrained project scheduling problem with mode dependent time lags", 44 (3), 257-273.

Siu, M., Lu, M. and AbouRizk, S., (2014), In Proceedings of the 2014 Winter Simulation Conference (WSC '14), "Bi-level project simulation methodology to integrate superintendent and project manager in decision making: shutdown/turnaround applications". IEEE Press, Piscataway, NJ, USA, 3363-3364.

Sjøbakk, B., Maria Kollberg Thomassen and Erlend Alfnes, (2013), International Workshop of Advanced Manufacturing and Automation, "Automation in the ETO Production Situation: The Case of a Norwegian Supplier of Ship Equipment", ISBN/ISBN2 978-82-321-0377-5/, ISSN/ISSN2 1892-8110.

Speranza, M. G, and C. Vercellis, (1992), European Journal of Operational Research, "Hierarchical models for multi-project planning and scheduling", 64, no. 2 (1993) 312-325.

SQL, (2014), http://www.microsoft.com/en-us/server-cloud/products/sql-server/

Beşikci,U., Bilge,U., Ulusoy,G., (2015), European Journal of Operational Research Multi-mode resource constrained multi-project scheduling and resource portfolio problem, , Volume 240, Issue 1, 1 January 2015, Pages 22-31, ISSN 0377-2217

Van Dijk, R. (2002), http://alexandria.tue.nl/repository/books/587366.pdf

Vanhoucke, M., (2013), "Project Management with Dynamic Scheduling, Baseline Scheduling, Risk Analysis and Project Control” 2nd ed. 2013, XVIII, 318 p. 123 illus.

Varma, V., Uzsoy, R., Pekny, J., Blau, G., (2007), Journal of Heuristics, "Lagrangian heuristics for scheduling new product development projects in the pharmaceutical industry", 13 (5), 403-433.

Wall, M., (1996), Ph.D. dissertation, "A Genetic Algorithm for Resource-Constrained Scheduling", Massachusetts Institute of Technology.

Wullink, G (2005), Ph.D. Thesis, "Resource Loading Under Uncertainty", Beta Research School for Operations Management and Logistics, The University of Twente, The Netherlands.

Wullink, G., Hans, E., Gademann, A., van Harten, A., (2004), International Journal of Production Research, "Scenario based approach for Flexible Resource Loading under Uncertainty", 42 (24), 50795098

$\mathrm{Xu}$, J. and Feng. C., (2014), The Scientific World Journal, "Multimode Resource-Constrained Multiple Project Scheduling Problem under Fuzzy Random Environment and Its Application to a Large Scale Hydropower Construction Project,", vol. 2014, Article ID 463692, 20 pages, 2014.

Xue, H., Wei, S., Wang, Y. (2010), Apperceiving Computing and Intelligence Analysis (ICACIA) International Conference, "Resource-constrained multi-project scheduling based on ant colony neural network", pp.179,182.

Yamashita, D., Amaral Armentano Vinícius, and Manuel Laguna, (2007), Journal of Scheduling, "Robust Optimization Models for Project Scheduling with Resource Availability Cost",10.1 (2007): 67. ProQuest. Web. 24 Jan. 2014. 
Yang, F., Jingang Liu (2012), European Journal of Operational Research, "Simulation-based transfer function modeling for transient analysis of general queueing systems", Volume 223, Issue 1, 16 November 2012, Pages 150-166, ISSN 0377-2217.

Zhang, L., Sun, R., (2011), Service Systems and Service Management (ICSSSM), 2011 8th International Conference, "An improvement of resource-constrained multi-project scheduling model based on priorityrule based heuristics", pp.1,5, 25-27 June 2011

Zhou, S., Ying Feng Zheng, Zhang Jiang, Meng Qiang Duan, (2013), Applied Mechanics and Materials, "Research of Scheduling Management in Warship Scheduled Repair Project", 409-410, 1564, DOI: 10.4028/www.scientific.net/AMM.409-410.1564

Zhu, G., Bard, J., Yu, G., (2006), INFORMS Journal on Computing "A branch-and-cut procedure for the multimode resource-constrained project-scheduling problem”, 377-390. 


\section{Appendix I: Server Side Database Tables}

\begin{tabular}{|l|l|l|l|}
\hline \multicolumn{1}{|c}{ Field } & \multicolumn{1}{c|}{ Data Type } & \multicolumn{1}{c|}{ Size } & \multicolumn{1}{c|}{ Description } \\
\hline ResourceID & Number & Integer & Resource ID \\
\hline Description & Text & 25 & Resource Description \\
\hline AvailabilityID & Number & Integer & Availability ID \\
\hline DirectCost & Number & Double & Direct Cost \\
\hline VariableCost & Number & Double & Variable cost/hour \\
\hline AdditionalTimeCost & Number & Double & Additional cost/hour \\
\hline MaintenanceCost & Number & Double & Maintenance cost/hour \\
\hline EnergyCost & Number & Double & Energy cost/hour \\
\hline Type & Text & 25 & Renewable and Nonrenewable \\
\hline
\end{tabular}

\begin{tabular}{|l|l|l|l|}
\hline \multicolumn{1}{|c|}{ Table AI.2: SIMU_RESOURCES_IDLE_TIMES } \\
\hline \multicolumn{1}{|c|}{ Field } & \multicolumn{1}{|c|}{ Data Type } & \multicolumn{1}{c|}{ Size } & \multicolumn{1}{c|}{ Description } \\
\hline ResourceID & Number & Integer & Resource ID \\
\hline StartTime & Date & 10 & Start time \\
\hline EndTime & Date & 10 & End time \\
\hline
\end{tabular}

\begin{tabular}{|l|l|l|l|}
\hline \multicolumn{1}{|c|}{ Table AI.3: SIMU_RESOURCES_NON_RENEWABLE } \\
\hline Field & \multicolumn{1}{|c|}{ Data Type } & \multicolumn{1}{c|}{ Size } & \multicolumn{1}{c|}{ Description } \\
\hline InventoryId & Number & Integer & Resource ID \\
\hline ResourceId & Number & Integer & Start time \\
\hline Quantity & Number & Double & End time \\
\hline InventoryType & Text & 15 & On hand/On order \\
\hline DeliveryDate & Date & 10 & Delivery Date \\
\hline
\end{tabular}

\begin{tabular}{|l|l|l|l|}
\hline \multicolumn{4}{|c|}{ Table AI.4: SIMU_RESOURCES_ADDITIONAL_TIMES } \\
\hline \multicolumn{1}{|c|}{ Field } & \multicolumn{1}{c|}{ Data Type } & \multicolumn{1}{c|}{ Size } & Description \\
\hline ResourceID & Number & Integer & Resource ID \\
\hline StartTime & Date & 10 & Start time \\
\hline EndTime & Date & 10 & End time \\
\hline
\end{tabular}




\begin{tabular}{|l|l|l|l|}
\hline \multicolumn{3}{c}{ Table AI.5: SIMU_RESOURCE_AVAILABILITY } \\
\hline \multicolumn{1}{|c|}{ Field } & \multicolumn{1}{c|}{ Data } & \multicolumn{1}{c|}{ Size } & \multicolumn{1}{c|}{ Description } \\
\hline A_W_SId & Text & 25 & Concatenated code between AvailabilityId, WeekDay, and \\
\hline AvailabilityId & Number & Integer & Availability ID \\
\hline WeekDay & Number & Integer & Week day \\
\hline StartTime & Date & 10 & Start Time Interval Partition \\
\hline EndTime & Date & 10 & End Time Interval Partition \\
\hline Type & Text & 25 & Idle, Regular or Additional \\
\hline
\end{tabular}

\begin{tabular}{|l|l|l|l|}
\hline \multicolumn{1}{|c|}{ Table AI.6: SIMU_RESOURCES_SKILLS } \\
\hline Field & \multicolumn{1}{c|}{ Data Type } & \multicolumn{1}{c|}{ Size } & \multicolumn{1}{c|}{ Description } \\
\hline ResourceID & Number & Integer & Resource ID \\
\hline SkillID & Number & Integer & Skill ID \\
\hline Rank & Number & Integer & 1-Excelent, 2-Good, 3-Reasonable, 4- \\
\hline
\end{tabular}

\begin{tabular}{|l|l|l|l|}
\hline \multicolumn{4}{|c|}{ Table AI.7: SIMU_TASKS } \\
\hline TaskID & Data Type & \multicolumn{1}{c|}{ Size } & Description \\
\hline Description & Number & Integer & Task ID \\
\hline NetDuration & Text & 25 & Task description \\
\hline Revenue & Number & Double & Remaining time to finish a task \\
\hline DueDate & Number & Double & Revenue \\
\hline PenaltyCost & Date & 10 & Due date \\
\hline Bonus & Number & Double & Penalty cost to no delivery on time \\
\hline
\end{tabular}

\begin{tabular}{|l|l|l|l|}
\hline \multicolumn{4}{|c|}{ Table AI.8: SIMU_TASK_MODES } \\
\hline \multicolumn{1}{|c|}{ Field } & Data Type & \multicolumn{1}{c|}{ Size } & \multicolumn{1}{c|}{ Description } \\
\hline TaskModeId & Text & 25 & Task Mode ID \\
\hline TaskID & Number & Integer & Task ID \\
\hline ModeID & Number & Integer & Mode ID \\
\hline Duration & Number & Double & Duration (hours) \\
\hline
\end{tabular}

\begin{tabular}{|l|l|l|l|}
\hline \multicolumn{4}{c}{ Table AI.9: SIMU_TASK_MODES_RESOURCES } \\
\hline \multicolumn{1}{|c|}{ Field } & \multicolumn{1}{c|}{ Data Type } & \multicolumn{1}{c|}{ Size } & Description \\
\hline TaskModeResourceId & Text & 25 & Task Mode ID \\
\hline TaskModeId & Text & 25 & Task ID \\
\hline Code & Number & Integer & Skill ID or Resource ID \\
\hline Consumption & Number & Double & Percentage required of a given resource \\
\hline Qty & Number & Integer & Resource quantity \\
\hline Type & Text & 25 & Skill or Resource \\
\hline
\end{tabular}


Table AI.10: SIMU_TASK_PRECEDENCE

\begin{tabular}{|l|l|l|l|}
\hline \multicolumn{1}{|c|}{ Field } & \multicolumn{1}{|c|}{ Data } & \multicolumn{1}{c|}{ Size } & \multicolumn{1}{c|}{ Description } \\
\hline Task_TaskPredId & Text & 25 & Concatenated code between Task and its Predecessor Task ID \\
\hline TaskID & Number & Integer & Task ID \\
\hline TaskPredID & Number & Integer & Task Predecessor ID \\
\hline TimeToRelease & Number & Double & Lag-time \\
\hline
\end{tabular}




\section{Appendix II: Data Files for Case Study 1}

\begin{tabular}{|c|c|c|c|c|c|c|c|c|c|}
\hline & \multicolumn{9}{|c|}{ Table AII.1: Resources Required by Each Task for Mode 1} \\
\hline & Task & BBF & $\mathbf{B E}$ & CMC & $\mathbf{C F}$ & CLB & $\mathbf{D L}$ & LB & $\mathbf{R R}$ \\
\hline 1 & A & & 4 & & & & & 8 & \\
\hline 2 & B1 & & 2 & & & & & 4 & \\
\hline 3 & $\mathrm{~B} 2$ & & 2 & 1 & & & & 4 & 2 \\
\hline 4 & B3 & & 1 & & & & & 4 & 2 \\
\hline 5 & B4 & & & 1 & & 2 & & 6 & \\
\hline 6 & B5 & 2 & & & & & & 4 & \\
\hline 7 & B6 & & & & 1 & & & 4 & \\
\hline 8 & B7 & & & 1 & & 3 & & 4 & \\
\hline 9 & B8 & & & & & & & 6 & \\
\hline 10 & B9 & 2 & & 1 & & & & 3 & \\
\hline 11 & B10 & & & & 1 & & & 4 & \\
\hline 12 & B11 & & & 1 & & 3 & & 4 & \\
\hline 13 & $\mathrm{C} 1$ & & 1 & & & & & 3 & 2 \\
\hline 14 & $\mathrm{C} 2$ & & & 1 & & 2 & & 4 & \\
\hline 15 & C3 & 2 & & & & & & 3 & \\
\hline 16 & $\mathrm{C} 4$ & & & & 1 & & & 4 & \\
\hline 17 & C5 & & & 1 & & 3 & & 4 & \\
\hline 18 & C6 & & & & & & & 5 & \\
\hline 19 & C7 & 2 & & 1 & & & & 3 & \\
\hline 20 & C8 & & & & 1 & & & 4 & \\
\hline 21 & C9 & & & 1 & & 3 & & 3 & \\
\hline 22 & D1 & & 1 & & & & & 2 & 1 \\
\hline 23 & D2 & & & 1 & & 2 & & 2 & 1 \\
\hline 24 & D3 & 1 & & & & & & 2 & \\
\hline 25 & D4 & & & & 1 & & & 4 & \\
\hline 26 & D5 & & & 1 & & 3 & & 2 & 1 \\
\hline 27 & D6 & & & & & & & 4 & \\
\hline 28 & D7 & 2 & & 1 & & & & 2 & \\
\hline 29 & D8 & & & & 1 & & & 4 & \\
\hline 30 & D9 & & & 1 & & 3 & & 2 & 1 \\
\hline 31 & E1 & & & & & & & & \\
\hline 31 & E2 & & & 1 & & & 1 & 6 & \\
\hline 33 & $\mathrm{~F}$ & & 4 & & & & & 8 & 4 \\
\hline \multicolumn{2}{|c|}{ Resource Limits } & 4 & 4 & 3 & 2 & 5 & 1 & 8 & 4 \\
\hline
\end{tabular}


Table AII.2: Duration and Resources Required by Tasks for Mode 2 


\begin{tabular}{|c|c|c|}
\hline \multicolumn{3}{|c|}{ Table AII.3: Resource Costs Example } \\
\hline Resource & Fixed Cost $\mathbf{( \$ )}$ & Variable Cost (USD/h) \\
\hline BBF & 1000 & 13 \\
\hline BE & 1000 & 38 \\
\hline CMC & 1000 & 150 \\
\hline CF & 1000 & 19.67 \\
\hline CLB & 1000 & 19.67 \\
\hline DL & 1000 & 85 \\
\hline LB & 1000 & 25 \\
\hline RR & 1000 & 94 \\
\hline
\end{tabular}

\begin{tabular}{|c|c|c|c|}
\hline \multicolumn{4}{|c|}{ Table AII.4: Due Dates, Penalty Costs and Bonus Example } \\
\hline Task & Due Date & Penalty Cost (USD/d) & Bonus (USD/d) \\
\hline A & $6 / 16 / 2015$ & 12000 & 6000 \\
\hline B1 & $6 / 26 / 2015$ & 12000 & 6000 \\
\hline $\mathrm{B} 2$ & $7 / 12 / 2015$ & 12000 & 6000 \\
\hline B3 & $7 / 28 / 2015$ & 12000 & 6000 \\
\hline B4 & $8 / 1 / 2015$ & 12000 & 6000 \\
\hline B5 & $9 / 21 / 2015$ & 12000 & 6000 \\
\hline B6 & $8 / 15 / 2015$ & 12000 & 6000 \\
\hline B7 & $9 / 14 / 2015$ & 12000 & 6000 \\
\hline B8 & $10 / 21 / 2015$ & 12000 & 6000 \\
\hline B9 & $11 / 20 / 2015$ & 12000 & 6000 \\
\hline B10 & $11 / 20 / 2015$ & 12000 & 6000 \\
\hline B11 & $11 / 26 / 2015$ & 12000 & 6000 \\
\hline $\mathrm{C} 1$ & $6 / 30 / 2015$ & 12000 & 6000 \\
\hline $\mathrm{C} 2$ & $7 / 2 / 2015$ & 12000 & 6000 \\
\hline C3 & $8 / 15 / 2015$ & 12000 & 6000 \\
\hline $\mathrm{C} 4$ & $8 / 15 / 2015$ & 12000 & 6000 \\
\hline $\mathrm{C} 5$ & $8 / 18 / 2015$ & 12000 & 6000 \\
\hline C6 & $9 / 20 / 2015$ & 12000 & 6000 \\
\hline C7 & $10 / 21 / 2015$ & 12000 & 6000 \\
\hline $\mathrm{C} 8$ & $10 / 21 / 2015$ & 12000 & 6000 \\
\hline C9 & $10 / 25 / 2015$ & 12000 & 6000 \\
\hline D1 & $10 / 28 / 2015$ & 12000 & 6000 \\
\hline D2 & $12 / 2 / 2015$ & 12000 & 6000 \\
\hline D3 & $12 / 5 / 2015$ & 12000 & 6000 \\
\hline D4 & $12 / 8 / 2015$ & 12000 & 6000 \\
\hline D5 & $1 / 10 / 2016$ & 12000 & 6000 \\
\hline D6 & $1 / 10 / 2016$ & 12000 & 6000 \\
\hline D7 & $1 / 13 / 2016$ & 12000 & 6000 \\
\hline D8 & $1 / 13 / 2016$ & 12000 & 6000 \\
\hline D9 & $1 / 25 / 2016$ & 12000 & 6000 \\
\hline
\end{tabular}




\begin{tabular}{|c|c|c|c|}
\hline $\mathrm{E} 1$ & $8 / 30 / 2015$ & 12000 & 6000 \\
\hline $\mathrm{E} 2$ & $9 / 2 / 2015$ & 12000 & 6000 \\
\hline $\mathrm{F}$ & $2 / 20 / 2016$ & 15000 & 6000 \\
\hline
\end{tabular}

Table AII.5: Detailed Schedule by STREAM for Scenario 3

\begin{tabular}{|c|c|c|c|c|}
\hline Task & Start Date & End Date & Due Date & Deviation (d) \\
\hline $\mathrm{A}$ & $5 / 28 / 2015$ & $6 / 15 / 2015$ & $6 / 16 / 2015$ & -0.29 \\
\hline B1 & $6 / 16 / 2015$ & $6 / 24 / 2015$ & $6 / 26 / 2015$ & -1.29 \\
\hline B2 & $6 / 25 / 2015$ & $7 / 13 / 2015$ & $7 / 12 / 2015$ & 1.71 \\
\hline B3 & $7 / 14 / 2015$ & $7 / 27 / 2015$ & $7 / 28 / 2015$ & -0.29 \\
\hline B4 & $7 / 28 / 2015$ & $7 / 30 / 2015$ & $8 / 1 / 2015$ & -1.29 \\
\hline B5 & $7 / 31 / 2015$ & $9 / 5 / 2015$ & $9 / 21 / 2015$ & -15.29 \\
\hline B6 & $7 / 31 / 2015$ & $8 / 18 / 2015$ & $8 / 15 / 2015$ & 3.71 \\
\hline B7 & $9 / 7 / 2015$ & $9 / 10 / 2015$ & $9 / 14 / 2015$ & -3.29 \\
\hline B8 & $9 / 11 / 2015$ & $10 / 17 / 2015$ & $10 / 21 / 2015$ & -3.29 \\
\hline B9 & $10 / 19 / 2015$ & $11 / 10 / 2015$ & $11 / 20 / 2015$ & -9.29 \\
\hline B10 & $10 / 19 / 2015$ & $11 / 10 / 2015$ & $11 / 20 / 2015$ & -9.29 \\
\hline B11 & $11 / 11 / 2015$ & $11 / 18 / 2015$ & $11 / 26 / 2015$ & -7.29 \\
\hline $\mathrm{C} 1$ & $6 / 16 / 2015$ & $6 / 29 / 2015$ & $6 / 30 / 2015$ & -0.29 \\
\hline $\mathrm{C} 2$ & $6 / 30 / 2015$ & $7 / 2 / 2015$ & $7 / 2 / 2015$ & 0.71 \\
\hline $\mathrm{C} 3$ & $7 / 3 / 2015$ & $8 / 8 / 2015$ & $8 / 15 / 2015$ & -6.29 \\
\hline $\mathrm{C} 4$ & $7 / 3 / 2015$ & $7 / 21 / 2015$ & $8 / 15 / 2015$ & -24.29 \\
\hline $\mathrm{C} 5$ & $8 / 10 / 2015$ & $8 / 13 / 2015$ & $8 / 18 / 2015$ & -4.29 \\
\hline C6 & $8 / 14 / 2015$ & $9 / 19 / 2015$ & $9 / 20 / 2015$ & -0.29 \\
\hline $\mathrm{C} 7$ & $9 / 21 / 2015$ & $10 / 13 / 2015$ & $10 / 21 / 2015$ & -7.29 \\
\hline $\mathrm{C} 8$ & $9 / 21 / 2015$ & $10 / 13 / 2015$ & $10 / 21 / 2015$ & -7.29 \\
\hline C9 & $10 / 14 / 2015$ & $10 / 21 / 2015$ & $10 / 25 / 2015$ & -3.29 \\
\hline D1 & $10 / 22 / 2015$ & $10 / 23 / 2015$ & $10 / 28 / 2015$ & -4.29 \\
\hline D2 & $10 / 26 / 2015$ & $10 / 26 / 2015$ & $12 / 2 / 2015$ & -36.29 \\
\hline D3 & $10 / 27 / 2015$ & $10 / 31 / 2015$ & $12 / 5 / 2015$ & -34.29 \\
\hline D4 & $11 / 2 / 2015$ & $11 / 3 / 2015$ & $12 / 8 / 2015$ & -34.29 \\
\hline D5 & $11 / 4 / 2015$ & $11 / 4 / 2015$ & $1 / 10 / 2016$ & -66.29 \\
\hline D6 & $11 / 5 / 2015$ & $11 / 9 / 2015$ & $1 / 10 / 2016$ & -61.29 \\
\hline D7 & $11 / 10 / 2015$ & $11 / 16 / 2015$ & $1 / 13 / 2016$ & -57.29 \\
\hline D8 & $11 / 10 / 2015$ & $11 / 12 / 2015$ & $1 / 13 / 2016$ & -61.29 \\
\hline D9 & $11 / 19 / 2015$ & $11 / 19 / 2015$ & $1 / 25 / 2016$ & -66.29 \\
\hline E1 & $5 / 28 / 2015$ & $8 / 25 / 2015$ & $8 / 30 / 2015$ & -4.29 \\
\hline E2 & $8 / 26 / 2015$ & $8 / 26 / 2015$ & $9 / 2 / 2015$ & -6.29 \\
\hline $\mathrm{F}$ & $11 / 20 / 2015$ & $11 / 27 / 2015$ & $2 / 20 / 2016$ & -84.29 \\
\hline
\end{tabular}




\begin{tabular}{|c|c|c|c|c|}
\hline \multicolumn{5}{|c|}{ Table AII.6: Detailed Scheduling by STREAM for Scenario 6} \\
\hline Task & Start Date & End Date & Due Date & Deviation (d) \\
\hline A & $5 / 28 / 2015$ & $6 / 15 / 2015$ & $6 / 16 / 2015$ & -0.29 \\
\hline B1 & $6 / 16 / 2015$ & $6 / 24 / 2015$ & $6 / 26 / 2015$ & -1.29 \\
\hline $\mathrm{B} 2$ & $6 / 25 / 2015$ & $7 / 13 / 2015$ & $7 / 12 / 2015$ & 1.71 \\
\hline B3 & $7 / 14 / 2015$ & $7 / 27 / 2015$ & $7 / 28 / 2015$ & -0.29 \\
\hline B4 & $7 / 28 / 2015$ & $8 / 4 / 2015$ & $8 / 1 / 2015$ & 3.71 \\
\hline B5 & $8 / 5 / 2015$ & $9 / 10 / 2015$ & $9 / 21 / 2015$ & -10.29 \\
\hline B6 & $8 / 5 / 2015$ & $9 / 1 / 2015$ & $8 / 15 / 2015$ & 17.71 \\
\hline B7 & $9 / 14 / 2015$ & $9 / 17 / 2015$ & $9 / 14 / 2015$ & 3.71 \\
\hline B8 & $9 / 18 / 2015$ & $10 / 24 / 2015$ & $10 / 21 / 2015$ & 3.71 \\
\hline B9 & $10 / 26 / 2015$ & $11 / 17 / 2015$ & $11 / 20 / 2015$ & -2.29 \\
\hline B10 & $10 / 26 / 2015$ & $11 / 26 / 2015$ & $11 / 20 / 2015$ & 6.71 \\
\hline B11 & $11 / 30 / 2015$ & $12 / 7 / 2015$ & $11 / 26 / 2015$ & 11.71 \\
\hline $\mathrm{C} 1$ & $6 / 16 / 2015$ & $6 / 29 / 2015$ & $6 / 30 / 2015$ & -0.29 \\
\hline $\mathrm{C} 2$ & $6 / 30 / 2015$ & $7 / 2 / 2015$ & $7 / 2 / 2015$ & 0.71 \\
\hline $\mathrm{C} 3$ & $7 / 3 / 2015$ & $8 / 8 / 2015$ & $8 / 15 / 2015$ & -6.29 \\
\hline $\mathrm{C} 4$ & $7 / 6 / 2015$ & $7 / 30 / 2015$ & $8 / 15 / 2015$ & -15.29 \\
\hline $\mathrm{C} 5$ & $8 / 10 / 2015$ & $8 / 17 / 2015$ & $8 / 18 / 2015$ & -0.29 \\
\hline C6 & $8 / 18 / 2015$ & $10 / 12 / 2015$ & $9 / 20 / 2015$ & 22.71 \\
\hline $\mathrm{C} 7$ & $10 / 13 / 2015$ & $11 / 4 / 2015$ & $10 / 21 / 2015$ & 14.71 \\
\hline $\mathrm{C} 8$ & $10 / 13 / 2015$ & $11 / 16 / 2015$ & $10 / 21 / 2015$ & 26.71 \\
\hline C9 & $11 / 17 / 2015$ & $11 / 24 / 2015$ & $10 / 25 / 2015$ & 30.71 \\
\hline D1 & $11 / 25 / 2015$ & $11 / 26 / 2015$ & $10 / 28 / 2015$ & 29.71 \\
\hline D2 & $11 / 30 / 2015$ & $11 / 30 / 2015$ & $12 / 2 / 2015$ & -1.29 \\
\hline D3 & $12 / 1 / 2015$ & $12 / 5 / 2015$ & $12 / 5 / 2015$ & 0.71 \\
\hline D4 & $12 / 7 / 2015$ & $12 / 8 / 2015$ & $12 / 8 / 2015$ & 0.71 \\
\hline D5 & $12 / 9 / 2015$ & $12 / 9 / 2015$ & $1 / 10 / 2016$ & -31.29 \\
\hline D6 & $12 / 10 / 2015$ & $12 / 14 / 2015$ & $1 / 10 / 2016$ & -26.29 \\
\hline D7 & $12 / 15 / 2015$ & $12 / 21 / 2015$ & $1 / 13 / 2016$ & -22.29 \\
\hline D8 & $12 / 15 / 2015$ & $12 / 17 / 2015$ & $1 / 13 / 2016$ & -26.29 \\
\hline D9 & $12 / 22 / 2015$ & $12 / 22 / 2015$ & $1 / 25 / 2016$ & -33.29 \\
\hline E1 & $5 / 28 / 2015$ & $8 / 25 / 2015$ & $8 / 30 / 2015$ & -4.29 \\
\hline E2 & $9 / 2 / 2015$ & $9 / 2 / 2015$ & $9 / 2 / 2015$ & 0.71 \\
\hline $\mathrm{F}$ & $12 / 23 / 2015$ & $12 / 30 / 2015$ & $2 / 20 / 2016$ & -51.29 \\
\hline
\end{tabular}




\section{Appendix III: Data Files for Case Study 2}

Table AIII.1: Task Description, Duration, Predecessors, and Resource Requirements

(Siu et. al, 2015)

\begin{tabular}{|c|c|c|c|c|}
\hline $\mathbf{T}$ & Description & Duration & Pred & Resources \\
\hline 1 & Install hex on external riser at cut line, approximately 30 & 50 & - & $\mathrm{A}[2] ; \mathrm{B}[2]$ \\
\hline 2 & Prejob meeting to install new reactor head & 1 & - & $\mathrm{C}[1] ; \mathrm{D}[6]$ \\
\hline 3 & Position crane and install rigging on new head & 8 & 2 & $\mathrm{C}[1] ; \mathrm{D}[6]$ \\
\hline 4 & Lift new head and swing amine unit & 4 & 3 & $\mathrm{C}[1] ; \mathrm{D}[6]$ \\
\hline 5 & Remove rigging and boom clear of work area & 4 & 6 & $\mathrm{C}[1] ; \mathrm{D}[6]$ \\
\hline 6 & Continue swing and lower new reactor head onto shell & 5 & 4 & $\mathrm{C}[1] ; \mathrm{D}[6]$ \\
\hline 7 & Hoard in decking on lower dipleg bracing back to shell & 10 & 5 & $\mathrm{E}[6]$ \\
\hline 8 & Fit and tack new head to existing reactor shell & 20 & 5 & $\mathrm{~A}[4] ; \mathrm{B}[4]$ \\
\hline 9 & Install landing from stairway to RX Platform 1 & 10 & - & $\mathrm{F}[3] ; \mathrm{G}[5]$ \\
\hline 10 & Install braces and structural section at platform & 30 & 5 & $\mathrm{~A}[3]$ \\
\hline 11 & Sign off to close regen manway MX-5 (plenum) & 1 & - & $\mathrm{H}[1] ; \mathrm{I}[1] ;$ \\
\hline 12 & Sign off to close regen manway MX-3 (plenum) & 1 & 11 & $\mathrm{H}[1] ; \mathrm{I}[1]$ \\
\hline 13 & Sign off to close regen OHL manway MX-4 (top OHL) & 1 & 12 & $\mathrm{H}[1] ; \mathrm{I}[1]$ \\
\hline 14 & Sign off to close regen OHL manway MX-6 (vertical section) & 1 & 13 & $\mathrm{H}[1] ; \mathrm{I}[1]$ \\
\hline 15 & Install bulkhead \#2 in reactor at lower elevation, also acc & 10 & 7 & $\mathrm{E}[6]$ \\
\hline 16 & Install landing from stairway to RX & 10 & 9 & $\mathrm{~F}[3] ; \mathrm{G}[5]$ \\
\hline 17 & Sign off to close regen OHL manway MX-7 (bottom section) & 1 & 14 & $\mathrm{H}[1] ; \mathrm{I}[1]$ \\
\hline 18 & Sign off to close regen OHL manway (west of stack valve) & 1 & 17 & $\mathrm{H}[1] ; \mathrm{I}[1]$ \\
\hline 19 & Install refractory plug_-regen manway MX-5 (plenum) & 4 & 18 & $\mathrm{~A}[1] ; \mathrm{B}[1]$ \\
\hline 20 & Install manway cover-regen manway MX-3 (plenum) & 3 & 18 & $\mathrm{~A}[2]$ \\
\hline 21 & Install manway cover - regen OHL manway (west of stack valve) & 3 & 20 & $\mathrm{~A}[2]$ \\
\hline 22 & Install refractory plug_regen OHL manway MX-4 (Top OHL) & 4 & 19 & $\mathrm{~A}[1] ; \mathrm{B}[1]$ \\
\hline 23 & Weld out new reactor head to existing reactor shell $(25 \%)$ & 20 & 8 & $\mathrm{~A}[1] ; \mathrm{B}[3]$ \\
\hline 24 & Lower riser into position, fit and tack & 20 & 15 & $\mathrm{~A}[4] ; \mathrm{B}[1]$ \\
\hline 25 & Install landing from stairway to RX Platform 2 & 10 & 16 & $\mathrm{~F}[3] ; \mathrm{G}[5]$ \\
\hline 26 & Install refractory plug —regen OHL manway MX-6 (vertical sec & 4 & 22 & $\mathrm{~A}[1] ; \mathrm{B}[1]$ \\
\hline 27 & Install refractory plug - regen OHL manway MX-7 (bottom secti & 4 & 26 & $\mathrm{~A}[1] ; \mathrm{B}[1]$ \\
\hline 28 & Install refractory—regen manway MX-5 (Plenum) & 6 & 27 & $\mathrm{~K}[3]$ \\
\hline 29 & Install landing from stairway to RX Platform 3 & 10 & 25 & $\mathrm{~F}[3] ; \mathrm{G}[5]$ \\
\hline 30 & Install refractory—regen OHL manway MX-4 (top OHL) & 6 & 28 & $\mathrm{~K}[3]$ \\
\hline 31 & Weld connect pressure tap piping from riser to shell, locat & 10 & 23 & $\mathrm{P}[2] ; \mathrm{Q}[1]$ \\
\hline 32 & Weld out new reactor head to existing reactor shell $(50 \%)$ & 20 & 23 & $\mathrm{~A}[3] ; \mathrm{B}[3]$ \\
\hline 33 & Weld out new riser duct to existing lower riser section & 40 & 24 & $\mathrm{~A}[2] ; \mathrm{B}[2]$ \\
\hline 34 & Install refractory—regen OHL manway MX-6 (vertical section) & 6 & 30 & $\mathrm{~K}[3]$ \\
\hline 35 & Install refractory—regen OHL manway MX-7 (bottom section) & 6 & 34 & $\mathrm{~K}[3]$ \\
\hline 36 & LPI weld connection pressure tap piping from riser to shell & 4 & 31 & $\mathrm{~L}[1]$ \\
\hline 37 & Refractory cure time - regen OHL manway MX-4, 5, 6, 7 & 12 & 35 & - \\
\hline 38 & Sign off install of pressure tap piping from riser to shell & 1 & 36 & $\mathrm{H}[1]$ \\
\hline
\end{tabular}




\begin{tabular}{|c|c|c|c|c|}
\hline 39 & Weld connect pressure tap piping from riser to shell, locat & 10 & 38 & $\mathrm{P}[2]$ \\
\hline 40 & Weld out new reactor head to existing reactor shell $(75 \%)$ & 20 & 32 & $\mathrm{~A}[3]$ \\
\hline 41 & Backgouge reactor weld of new shell to existing shell & 10 & 32 & $\mathrm{~A}[4]$ \\
\hline 42 & Install cover plate—regen manway MX-5 (plenum) & 2 & 37 & $\mathrm{~A}[2]$ \\
\hline 43 & LPI weld connection pressure tap piping from riser to shell & 4 & 39 & $\mathrm{~L}[1]$ \\
\hline 44 & Install cover plate—regen OHL manway MX-4 (top OHL) & 2 & 42 & $\mathrm{~A}[2]$ \\
\hline 45 & Install cover plate-regen OHL manway MX-6 (vertical section & 2 & 44 & $\mathrm{~A}[2]$ \\
\hline 46 & Sign off install of pressure tap piping from riser to shell & 1 & 43 & $\mathrm{H}[1]$ \\
\hline 47 & Weld connect TI piping from riser to shell, located above 1 & 10 & 46 & $\mathrm{P}[2]$ \\
\hline 48 & Weld inside of new shell to existing shell & 20 & 41 & $\mathrm{~A}[4]$ \\
\hline 49 & Install cover plate-regen OHL manway MX-7 (bottom section) & 2 & 45 & $\mathrm{~A}[2]$ \\
\hline 50 & LPI weld connection TI piping from riser to shell, located & 4 & 47 & $\mathrm{~L}[1]$ \\
\hline 51 & Final NDE on riser weld & 4 & 33 & M[1] \\
\hline 52 & Weld out new reactor head to existing reactor shell $(100 \%)$ & 20 & 40 & $\mathrm{~A}[3]$ \\
\hline 53 & Sign off installation of TI piping from riser to shell, loc & 1 & 50 & $\mathrm{H}[1]$ \\
\hline 54 & Install OD riser hex mesh at cut line, approximately $15 \mathrm{squ}$ & 25 & 51 & $\mathrm{~A}[2]$ \\
\hline 55 & Phase array weld of new reactor head to existing reactor sh & 10 & 52 & $\mathrm{~N}[1]$ \\
\hline 56 & Install refractory in hex on external riser weld location, & 40 & 54 & $\mathrm{~K}[6]$ \\
\hline 57 & Ball test primary cyclones and sign off to install riser ma & 2 & 29 & $\mathrm{H}[1]$ \\
\hline 58 & Layout and install refractory anchors on reactor head weldo & 20 & 55 & $\mathrm{~A}[2]$ \\
\hline 59 & Buff shell weld for painting & 10 & 55 & $\mathrm{O}[2]$ \\
\hline 60 & Install bridge steel from new stairway to reactor head $30-$ & 30 & - & $\mathrm{F}[3]$ \\
\hline 61 & Install riser manway and seal weld & 8 & 57 & $\mathrm{~A}[2]$ \\
\hline 62 & Paint shell weld for painting & 5 & 59 & $\mathrm{O}[2]$ \\
\hline 63 & Install platform 1 , section $0-90$ from $\mathrm{RX}$ to reg. & 30 & 10 & $\mathrm{~A}[3]$ \\
\hline 64 & NDE on riser manway cover & 1 & 61 & $\mathrm{~A}[2] ; \mathrm{B}[1]$ \\
\hline 65 & Remove scaffold from ACB & 6 & 64 & $\mathrm{E}[5]$ \\
\hline 66 & Final cleaning of ACB & 4 & 65 & $\mathrm{~A}[2]$ \\
\hline 67 & Install refractory on new reactor head to existing shell we & 20 & 58 & $\mathrm{~K}[1]$ \\
\hline 68 & Sign off to close reactor $\mathrm{MW}-\mathrm{MX}-4,5,6,7,8,9(\mathrm{ACB})$ & 4 & 66 & $\mathrm{H}[1]$ \\
\hline 69 & Close reactor MW-MX-4 (ACB)—install refractory plug & 6 & 68 & $\mathrm{~A}[1]$ \\
\hline 70 & Close reactor MW-MX-5 (ACB) — install refractory plug & 6 & 69 & $\mathrm{~A}[1]$ \\
\hline 71 & Close reactor MW—MX-4 (ACB) — install refractory in & 6 & 69 & $\mathrm{~K}[4]$ \\
\hline 72 & Close reactor MW-MX-6 (ACB) — install refractory plug & 6 & 70 & $\mathrm{~A}[1]$ \\
\hline 73 & Close reactor MW-MX-5 (ACB) - install refractory in & 6 & 71 & $\mathrm{~K}[4]$ \\
\hline 74 & Sign off refractory installation on riser OD 2 & 2 & 56 & $\mathrm{H}[1]$ \\
\hline 75 & Close reactor MW-MX-7 (ACB) — install refractory plug & 6 & 72 & $\mathrm{~A}[1]$ \\
\hline 76 & Close reactor MW—MX-6 (ACB) — install refractory in & 6 & 73 & $\mathrm{~K}[4]$ \\
\hline 77 & Riser-remove all internal scaffolding in riser & 5 & - & $\mathrm{E}[4]$ \\
\hline 78 & Riser-weld on riser manway & 12 & 77 & $\mathrm{~A}[1]$ \\
\hline 79 & Close reactor MW-MX-8 (ACB) — install refractory plug & 6 & 75 & $\mathrm{~A}[1]$ \\
\hline 80 & Close reactor MW-MX-7 (ACB) — install refractory in & 6 & 76 & $\mathrm{~K}[4]$ \\
\hline 81 & Close reactor MW-MX-9 (ACB) — install refractory plug & 6 & 79 & $\mathrm{~A}[1]$ \\
\hline 82 & Close reactor MW-MX-8 (ACB) - install refractory in & 6 & 80 & $\mathrm{~K}[4]$ \\
\hline
\end{tabular}




\begin{tabular}{|c|c|c|c|c|}
\hline 83 & Riser-NDE on riser manway weld & 1 & 78 & $\mathrm{~A}[1]$ \\
\hline 84 & Close reactor $\mathrm{MW}-\mathrm{MX}-9(\mathrm{ACB})$ - install refractory in & 6 & 82 & $\mathrm{~K}[4]$ \\
\hline 85 & Close reactor $\mathrm{MW}-\mathrm{MW}-\mathrm{MX}-4,5,6,7,8,9(\mathrm{ACB})$ - cure & 12 & 84 & - \\
\hline 86 & Install platform 2, section 0-90 from RX to reg. & 30 & 63 & $\mathrm{~A}[3]$ \\
\hline 87 & Close reactor MW-MX-4 (ACB)—close manway cover plate & 3 & 85 & $\mathrm{~A}[2]$ \\
\hline 88 & Close reactor MW-MX-5 (ACB) — close manway cover plate & 3 & 87 & $\mathrm{~A}[2]$ \\
\hline 89 & Close reactor MW-MX-6 (ACB) — close manway cover plate & 3 & 88 & $\mathrm{~A}[2]$ \\
\hline 90 & Close reactor MW-MX-7 (ACB) — close manway cover plate & 3 & 89 & $\mathrm{~A}[2]$ \\
\hline 91 & Close reactor MW—MX-8 (ACB)—close manway cover plate & 3 & 90 & $\mathrm{~A}[2]$ \\
\hline 92 & Close reactor MW-MX-9 (ACB) — close manway cover plate & 3 & 91 & $\mathrm{~A}[2]$ \\
\hline 93 & Sign off to close reactor MW-MX-1 (shell) & 1 & - & $\mathrm{H}[1]$ \\
\hline 94 & Sign off to close reactor MW—big MW & 1 & - & $\mathrm{H}[1]$ \\
\hline 95 & Sign off to close reactor MW-stripper cone & 1 & - & $\mathrm{H}[1]$ \\
\hline 96 & Close reactor MW-MX-1 (shell) & 5 & 93 & $\mathrm{~A}[2]$ \\
\hline 97 & Close reactor MW-MX-big MW & 6 & 94 & $\mathrm{~A}[4]$ \\
\hline 98 & Install refractory plug_-stripper cone manway & 4 & 95 & $\mathrm{~A}[1]$ \\
\hline 99 & Install refractory-stripper cone manway & 6 & 98 & $\mathrm{~K}[3]$ \\
\hline 10 & Install cover plate - stripper cone manway & 2 & 99 & $\mathrm{~A}[2]$ \\
\hline 10 & Sign off to close reactor MW-MX-10 (plenum) & 1 & - & $\mathrm{H}[1]$ \\
\hline 10 & Sign off to close reactor MW-MX-11 (plenum) & 1 & - & $\mathrm{H}[1]$ \\
\hline 10 & Close reactor MW-MX-10 (Plenum) — close manway cover & 3 & 101 & $\mathrm{~A}[2]$ \\
\hline 10 & Close reactor MW-MX-11 (Plenum) —install refractory plu0g & 6 & 102 & $\mathrm{~A}[1]$ \\
\hline 10 & Close reactor MW-MX-11 (Plenum) —install refractory in & 5 & 104 & $\mathrm{~K}[4]$ \\
\hline 10 & Close reactor MW-MX-11 (Plenum)—close manway cover & 3 & 105 & $\mathrm{~A}[2]$ \\
\hline 10 & Install platform 3, section 0-90 from RX to reg. & 30 & 86 & $\mathrm{~A}[3]$ \\
\hline
\end{tabular}


Table AIII.2: Analytical solution - Task finish time (Siu et. al, 2015)

\begin{tabular}{|c|c|c|}
\hline Task & Description & Finish Time \\
\hline 1 & Install hex on external riser at cut line, approximately 30 & 50 \\
\hline 2 & Prejob meeting to install new reactor head & 1 \\
\hline 3 & Position crane and install rigging on new head & 9 \\
\hline 4 & Lift new head and swing amine unit & 13 \\
\hline 5 & Remove rigging and boom clear of work area & 22 \\
\hline 6 & Continue swing and lower new reactor head onto shell & 18 \\
\hline 7 & Hoard in decking on lower dipleg bracing back to shell & 32 \\
\hline 8 & Fit and tack new head to existing reactor shell & 42 \\
\hline 9 & Install landing from stairway to RX Platform 1 & 10 \\
\hline 10 & Install braces and structural section at platform & 52 \\
\hline 11 & Sign off to close regen manway MX-5 (plenum) & 1 \\
\hline 12 & Sign off to close regen manway MX-3 (plenum) & 2 \\
\hline 13 & Sign off to close regen OHL manway MX-4 (top OHL) & 3 \\
\hline 14 & Sign off to close regen OHL manway MX-6 (vertical section) & 4 \\
\hline 15 & Install bulkhead \#2 in reactor at lower elevation, also acc & 42 \\
\hline 16 & Install landing from stairway to RX & 20 \\
\hline 17 & Sign off to close regen OHL manway MX-7 (bottom section) & 5 \\
\hline 18 & Sign off to close regen OHL manway (west of stack valve) & 6 \\
\hline 19 & Install refractory plug_-regen manway MX-5 (plenum) & 10 \\
\hline 20 & Install manway cover-regen manway MX-3 (plenum) & 9 \\
\hline 21 & Install manway cover - regen OHL manway (west of stack valve) & 12 \\
\hline 22 & Install refractory plug_-regen OHL manway MX-4 (Top OHL) & 14 \\
\hline 23 & Weld out new reactor head to existing reactor shell $(25 \%)$ & 62 \\
\hline 24 & Lower riser into position, fit and tack & 62 \\
\hline 25 & Install landing from stairway to RX Platform 2 & 30 \\
\hline 26 & Install refractory plug - regen OHL manway MX-6 (vertical sec & 18 \\
\hline 27 & Install refractory plug-regen OHL manway MX-7 (bottom secti & 22 \\
\hline 28 & Install refractory-regen manway MX-5 (Plenum) & 28 \\
\hline 29 & Install landing from stairway to RX Platform 3 & 40 \\
\hline 30 & Install refractory-regen OHL manway MX-4 (top OHL) & 34 \\
\hline 31 & Weld connect pressure tap piping from riser to shell, locat & 72 \\
\hline 32 & Weld out new reactor head to existing reactor shell (50\%) & 82 \\
\hline 33 & Weld out new riser duct to existing lower riser section & 102 \\
\hline 34 & Install refractory - regen OHL manway MX-6 (vertical section) & 40 \\
\hline 35 & Install refractory-regen OHL manway MX-7 (bottom section) & 46 \\
\hline 36 & LPI weld connection pressure tap piping from riser to shell & 76 \\
\hline 37 & Refractory cure time - regen OHL manway MX-4, 5, 6, 7 & 58 \\
\hline 38 & Sign off install of pressure tap piping from riser to shell & 77 \\
\hline 39 & Weld connect pressure tap piping from riser to shell, locat & 87 \\
\hline 40 & Weld out new reactor head to existing reactor shell (75\%) & 102 \\
\hline 41 & Backgouge reactor weld of new shell to existing shell & 92 \\
\hline
\end{tabular}




\begin{tabular}{|c|c|c|}
\hline 42 & Install cover plate-regen manway MX-5 (plenum) & 60 \\
\hline 43 & LPI weld connection pressure tap piping from riser to shell & 91 \\
\hline 44 & Install cover plate - regen OHL manway MX-4 (top OHL) & 62 \\
\hline 45 & Install cover plate-regen OHL manway MX-6 (vertical section & 64 \\
\hline 46 & Sign off install of pressure tap piping from riser to shell & 92 \\
\hline 47 & Weld connect TI piping from riser to shell, located above 1 & 102 \\
\hline 48 & Weld inside of new shell to existing shell & 112 \\
\hline 49 & Install cover plate-regen OHL manway MX-7 (bottom section) & 66 \\
\hline 50 & LPI weld connection TI piping from riser to shell, located & 106 \\
\hline 51 & Final NDE on riser weld & 106 \\
\hline 52 & Weld out new reactor head to existing reactor shell $(100 \%)$ & 122 \\
\hline 53 & Sign off installation of TI piping from riser to shell, loc & 107 \\
\hline 54 & Install OD riser hex mesh at cut line, approximately $15 \mathrm{squ}$ & 131 \\
\hline 55 & Phase array weld of new reactor head to existing reactor sh & 132 \\
\hline 56 & Install refractory in hex on external riser weld location, & 171 \\
\hline 57 & Ball test primary cyclones and sign off to install riser ma & 42 \\
\hline 58 & Layout and install refractory anchors on reactor head weldo & 152 \\
\hline 59 & Buff shell weld for painting & 142 \\
\hline 60 & Install bridge steel from new stairway to reactor head $30-$ & 30 \\
\hline 61 & Install riser manway and seal weld & 50 \\
\hline 62 & Paint shell weld for painting & 147 \\
\hline 63 & Install platform 1 , section $0-90$ from $\mathrm{RX}$ to reg. & 82 \\
\hline 64 & NDE on riser manway cover & 51 \\
\hline 65 & Remove scaffold from ACB & 57 \\
\hline 66 & Final cleaning of ACB & 61 \\
\hline 67 & Install refractory on new reactor head to existing shell we & 172 \\
\hline 68 & Sign off to close reactor $\mathrm{MW}-\mathrm{MX}-4,5,6,7,8,9(\mathrm{ACB})$ & 65 \\
\hline 69 & Close reactor MW—MX-4 (ACB)—install refractory plug & 71 \\
\hline 70 & Close reactor MW—MX-5 (ACB)—install refractory plug & 77 \\
\hline 71 & Close reactor MW-MX-4 (ACB) — install refractory in manway ne & 77 \\
\hline 72 & Close reactor MW—MX-6 (ACB)—install refractory plug & 83 \\
\hline 73 & Close reactor MW-MX-5 (ACB) — install refractory in manway ne & 83 \\
\hline 74 & Sign off refractory installation on riser OD 2 & 173 \\
\hline 75 & Close reactor MW—MX-7 (ACB)—install refractory plug & 89 \\
\hline 76 & Close reactor MW-MX-6 (ACB) — install refractory in manway ne & 89 \\
\hline 77 & Riser-remove all internal scaffolding in riser & 5 \\
\hline 78 & Riser—weld on riser manway & 17 \\
\hline 79 & Close reactor MW-MX-8 (ACB) — install refractory plug & 95 \\
\hline 80 & Close reactor MW_-MX-7 (ACB) — install refractory in manway ne & 95 \\
\hline 81 & Close reactor MW—MX-9 (ACB)—install refractory plug & 101 \\
\hline 82 & Close reactor MW-MX-8 (ACB) — install refractory in manway ne & 101 \\
\hline 83 & Riser-NDE on riser manway weld & 18 \\
\hline 84 & Close reactor MW-MX-9 (ACB) — install refractory in manway ne & 107 \\
\hline 85 & Close reactor $\mathrm{MW}-\mathrm{MW}-\mathrm{MX}-4,5,6,7,8,9(\mathrm{ACB})$ - cure time & 119 \\
\hline
\end{tabular}




\begin{tabular}{|c|c|c|}
\hline 86 & Install platform 2 , section $0-90$ from $\mathrm{RX}$ to reg. & 112 \\
\hline 87 & Close reactor MW-MX-4 (ACB) — close manway cover plate & 122 \\
\hline 88 & Close reactor MW-MX-5 (ACB) — close manway cover plate & 125 \\
\hline 89 & Close reactor MW-MX-6 (ACB)—close manway cover plate & 128 \\
\hline 90 & Close reactor MW-MX-7 (ACB)—close manway cover plate & 131 \\
\hline 91 & Close reactor MW-MX-8 (ACB) — close manway cover plate & 134 \\
\hline 92 & Close reactor MW-MX-9 (ACB) — close manway cover plate & 137 \\
\hline 93 & Sign off to close reactor MW-MX-1 (shell) & 5 \\
\hline 94 & Sign off to close reactor MW-big MW & 4 \\
\hline 95 & Sign off to close reactor MW-stripper cone & 1 \\
\hline 96 & Close reactor MW-MX-1 (shell) & 10 \\
\hline 97 & Close reactor MW-MX-big MW & 10 \\
\hline 98 & Install refractory plug_-stripper cone manway & 5 \\
\hline 99 & Install refractory-stripper cone manway & 11 \\
\hline 100 & Install cover plate-stripper cone manway & 13 \\
\hline 101 & Sign off to close reactor MW-MX-10 (plenum) & 3 \\
\hline 102 & Sign off to close reactor MW-MX-11 (plenum) & 2 \\
\hline 103 & Close reactor MW-MX-10 (Plenum) — close manway cover plate & 6 \\
\hline 104 & Close reactor MW—MX-11 (Plenum) —install refractory plu0g & 8 \\
\hline 105 & Close reactor MW-MX-11 (Plenum) —install refractory in man & 13 \\
\hline 106 & Close reactor MW-MX-11 (Plenum)—close manway cover plate & 16 \\
\hline 107 & Install platform 3, section 0-90 from RX to reg. & 142 \\
\hline
\end{tabular}

Table AIII.3: Solution results

\begin{tabular}{|c|c|c|c|c|c|c|c|c|}
\hline Task & \multicolumn{2}{|c}{ Run 1 } & \multicolumn{2}{c|}{ Run 2 } & \multicolumn{2}{c|}{ Run 3 } & \multicolumn{2}{c|}{ Run 4 } \\
\hline & Time (h) & Diff. & Time & Diff. & Time & Diff. & Time & Diff. \\
\hline 1 & 50 & 0 & 50 & 0 & 50 & 0 & 50 & 0 \\
\hline 2 & 1 & 0 & 1 & 0 & 1 & 0 & 1 & 0 \\
\hline 3 & 9 & 0 & 9 & 0 & 9 & 0 & 9 & 0 \\
\hline 4 & 13 & 0 & 13 & 0 & 13 & 0 & 13 & 0 \\
\hline 5 & 22 & 0 & 22 & 0 & 22 & 0 & 22 & 0 \\
\hline 6 & 18 & 0 & 18 & 0 & 18 & 0 & 18 & 0 \\
\hline 7 & 32 & 0 & 32 & 0 & 32 & 0 & 32 & 0 \\
\hline 8 & 42 & 0 & 42 & 0 & 42 & 0 & 42 & 0 \\
\hline 9 & 10 & 0 & 10 & 0 & 10 & 0 & 10 & 0 \\
\hline 10 & 52 & 0 & 52 & 0 & 70 & 18 & 52 & 0 \\
\hline 11 & 1 & 0 & 1 & 0 & 1 & 0 & 1 & 0 \\
\hline 12 & 2 & 0 & 2 & 0 & 4 & 2 & 2 & 0 \\
\hline 13 & 3 & 0 & 3 & 0 & 5 & 2 & 3 & 0 \\
\hline 14 & 4 & 0 & 4 & 0 & 7 & 3 & 4 & 0 \\
\hline 15 & 42 & 0 & 42 & 0 & 42 & 0 & 42 & 0 \\
\hline 16 & 20 & 0 & 20 & 0 & 20 & 0 & 20 & 0 \\
\hline 17 & 5 & 0 & 5 & 0 & 9 & 4 & 5 & 0 \\
\hline
\end{tabular}




\begin{tabular}{|c|c|c|c|c|c|c|c|c|}
\hline 18 & 6 & 0 & 6 & 0 & 10 & 4 & 6 & 0 \\
\hline 19 & 10 & 0 & 10 & 0 & 14 & 4 & 10 & 0 \\
\hline 20 & 9 & 0 & 9 & 0 & 13 & 4 & 9 & 0 \\
\hline 21 & 12 & 0 & 12 & 0 & 17 & 5 & 12 & 0 \\
\hline 22 & 14 & 0 & 14 & 0 & 18 & 4 & 14 & 0 \\
\hline 23 & 62 & 0 & 62 & 0 & 62 & 0 & 62 & 0 \\
\hline 24 & 62 & 0 & 62 & 0 & 62 & 0 & 62 & 0 \\
\hline 25 & 30 & 0 & 30 & 0 & 30 & 0 & 30 & 0 \\
\hline 26 & 18 & 0 & 18 & 0 & 22 & 4 & 18 & 0 \\
\hline 27 & 22 & 0 & 22 & 0 & 26 & 4 & 22 & 0 \\
\hline 28 & 28 & 0 & 28 & 0 & 32 & 4 & 28 & 0 \\
\hline 29 & 40 & 0 & 40 & 0 & 40 & 0 & 40 & 0 \\
\hline 30 & 34 & 0 & 34 & 0 & 38 & 4 & 34 & 0 \\
\hline 31 & 72 & 0 & 72 & 0 & 72 & 0 & 72 & 0 \\
\hline 32 & 82 & 0 & 82 & 0 & 82 & 0 & 82 & 0 \\
\hline 33 & 102 & 0 & 102 & 0 & 102 & 0 & 102 & 0 \\
\hline 34 & 40 & 0 & 40 & 0 & 44 & 4 & 40 & 0 \\
\hline 35 & 46 & 0 & 46 & 0 & 50 & 4 & 46 & 0 \\
\hline 36 & 76 & 0 & 76 & 0 & 76 & 0 & 76 & 0 \\
\hline 37 & 58 & 0 & 58 & 0 & 62 & 4 & 58 & 0 \\
\hline 38 & 77 & 0 & 77 & 0 & 77 & 0 & 77 & 0 \\
\hline 39 & 87 & 0 & 87 & 0 & 87 & 0 & 87 & 0 \\
\hline 40 & 102 & 0 & 102 & 0 & 102 & 0 & 102 & 0 \\
\hline 41 & 92 & 0 & 92 & 0 & 92 & 0 & 92 & 0 \\
\hline 42 & 60 & 0 & 60 & 0 & 64 & 4 & 60 & 0 \\
\hline 43 & 91 & 0 & 91 & 0 & 91 & 0 & 91 & 0 \\
\hline 44 & 62 & 0 & 62 & 0 & 66 & 4 & 62 & 0 \\
\hline 45 & 64 & 0 & 64 & 0 & 68 & 4 & 64 & 0 \\
\hline 46 & 92 & 0 & 92 & 0 & 92 & 0 & 92 & 0 \\
\hline 47 & 102 & 0 & 102 & 0 & 102 & 0 & 102 & 0 \\
\hline 48 & 112 & 0 & 112 & 0 & 112 & 0 & 112 & 0 \\
\hline 49 & 66 & 0 & 66 & 0 & 70 & 4 & 66 & 0 \\
\hline 50 & 106 & 0 & 106 & 0 & 106 & 0 & 106 & 0 \\
\hline 51 & 106 & 0 & 106 & 0 & 106 & 0 & 106 & 0 \\
\hline 52 & 122 & 0 & 122 & 0 & 122 & 0 & 122 & 0 \\
\hline 53 & 107 & 0 & 107 & 0 & 107 & 0 & 107 & 0 \\
\hline 54 & 131 & 0 & 131 & 0 & 131 & 0 & 131 & 0 \\
\hline 55 & 132 & 0 & 132 & 0 & 132 & 0 & 132 & 0 \\
\hline 56 & 171 & 0 & 171 & 0 & 171 & 0 & 171 & 0 \\
\hline 57 & 42 & 0 & 42 & 0 & 42 & 0 & 42 & 0 \\
\hline 58 & 152 & 0 & 152 & 0 & 152 & 0 & 152 & 0 \\
\hline 59 & 142 & 0 & 142 & 0 & 142 & 0 & 142 & 0 \\
\hline 60 & 30 & 0 & 30 & 0 & 70 & 40 & 30 & 0 \\
\hline 61 & 50 & 0 & 50 & 0 & 58 & 8 & 50 & 0 \\
\hline
\end{tabular}




\begin{tabular}{|c|c|c|c|c|c|c|c|c|}
\hline 62 & 147 & 0 & 147 & 0 & 147 & 0 & 147 & 0 \\
\hline 63 & 82 & 0 & 82 & 0 & 100 & 18 & 82 & 0 \\
\hline 64 & 51 & 0 & 51 & 0 & 59 & 8 & 51 & 0 \\
\hline 65 & 57 & 0 & 57 & 0 & 65 & 8 & 57 & 0 \\
\hline 66 & 61 & 0 & 61 & 0 & 69 & 8 & 61 & 0 \\
\hline 67 & 172 & 0 & 172 & 0 & 172 & 0 & 172 & 0 \\
\hline 68 & 65 & 0 & 65 & 0 & 73 & 8 & 65 & 0 \\
\hline 69 & 71 & 0 & 71 & 0 & 79 & 8 & 71 & 0 \\
\hline 70 & 77 & 0 & 77 & 0 & 85 & 8 & 77 & 0 \\
\hline 71 & 77 & 0 & 77 & 0 & 85 & 8 & 77 & 0 \\
\hline 72 & 83 & 0 & 83 & 0 & 91 & 8 & 83 & 0 \\
\hline 73 & 83 & 0 & 83 & 0 & 91 & 8 & 83 & 0 \\
\hline 74 & 173 & 0 & 173 & 0 & 173 & 0 & 173 & 0 \\
\hline 75 & 89 & 0 & 89 & 0 & 97 & 8 & 89 & 0 \\
\hline 76 & 89 & 0 & 89 & 0 & 97 & 8 & 89 & 0 \\
\hline 77 & 5 & 0 & 5 & 0 & 5 & 0 & 5 & 0 \\
\hline 78 & 17 & 0 & 17 & 0 & 17 & 0 & 17 & 0 \\
\hline 79 & 95 & 0 & 95 & 0 & 103 & 8 & 95 & 0 \\
\hline 80 & 95 & 0 & 95 & 0 & 103 & 8 & 95 & 0 \\
\hline 81 & 101 & 0 & 101 & 0 & 109 & 8 & 101 & 0 \\
\hline 82 & 101 & 0 & 101 & 0 & 109 & 8 & 101 & 0 \\
\hline 83 & 18 & 0 & 18 & 0 & 18 & 0 & 18 & 0 \\
\hline 84 & 107 & 0 & 107 & 0 & 115 & 8 & 107 & 0 \\
\hline 85 & 119 & 0 & 119 & 0 & 127 & 8 & 119 & 0 \\
\hline 86 & 112 & 0 & 112 & 0 & 130 & 18 & 112 & 0 \\
\hline 87 & 122 & 0 & 122 & 0 & 130 & 8 & 122 & 0 \\
\hline 88 & 125 & 0 & 125 & 0 & 133 & 8 & 125 & 0 \\
\hline 89 & 128 & 0 & 128 & 0 & 136 & 8 & 128 & 0 \\
\hline 90 & 131 & 0 & 131 & 0 & 139 & 8 & 131 & 0 \\
\hline 91 & 134 & 0 & 134 & 0 & 142 & 8 & 134 & 0 \\
\hline 92 & 137 & 0 & 137 & 0 & 145 & 8 & 137 & 0 \\
\hline 93 & 3 & -2 & 5 & 0 & 11 & 6 & 1 & -4 \\
\hline 94 & 4 & 0 & 4 & 0 & 8 & 4 & 1 & -3 \\
\hline 95 & 1 & 0 & 1 & 0 & 2 & 1 & 1 & 0 \\
\hline 96 & 8 & -2 & 10 & 0 & 16 & 6 & 6 & -4 \\
\hline 97 & 10 & 0 & 10 & 0 & 14 & 4 & 7 & -3 \\
\hline 98 & 5 & 0 & 5 & 0 & 6 & 1 & 5 & 0 \\
\hline 99 & 11 & 0 & 11 & 0 & 12 & 1 & 11 & 0 \\
\hline 100 & 13 & 0 & 13 & 0 & 15 & 2 & 13 & 0 \\
\hline 101 & 5 & 2 & 3 & 0 & 6 & 3 & 1 & -2 \\
\hline 102 & 2 & 0 & 2 & 0 & 3 & 1 & 1 & -1 \\
\hline 103 & 8 & 2 & 6 & 0 & 9 & 3 & 4 & -2 \\
\hline 104 & 8 & 0 & 8 & 0 & 9 & 1 & 7 & -1 \\
\hline 105 & 13 & 0 & 13 & 0 & 14 & 1 & 12 & -1 \\
\hline
\end{tabular}




\begin{tabular}{|c|c|c|c|c|c|c|c|c|}
\hline 106 & 16 & 0 & 16 & 0 & 17 & 1 & 15 & -1 \\
\hline 107 & 142 & 0 & 142 & 0 & 160 & 18 & 142 & 0 \\
\hline TPD & $\mathbf{1 7 3}$ & & $\mathbf{1 7 3}$ & & $\mathbf{1 7 3}$ & & $\mathbf{1 7 3}$ & \\
\hline
\end{tabular}

Table AIII.4: Resource Utilization (STREAM Run 2)

\begin{tabular}{|c|c|c|c|}
\hline & Resource & Idle time \% & Working time \% \\
\hline 1 & A1 & 18.50 & 81.50 \\
\hline 2 & $\mathrm{~A} 2$ & 18.50 & 81.50 \\
\hline 3 & A3 & 27.17 & 72.83 \\
\hline 4 & A4 & 26.01 & 73.99 \\
\hline 5 & A5 & 26.59 & 73.41 \\
\hline 6 & A6 & 25.43 & 74.57 \\
\hline 7 & A7 & 27.17 & 72.83 \\
\hline 8 & A8 & 39.31 & 60.69 \\
\hline 9 & A9 & 55.49 & 44.51 \\
\hline 10 & A10 & 55.49 & 44.51 \\
\hline 11 & A11 & 60.69 & 39.31 \\
\hline 12 & A12 & 68.21 & 31.79 \\
\hline 13 & A13 & 69.36 & 30.64 \\
\hline 14 & A14 & 93.06 & 6.94 \\
\hline 21 & B1 & 18.50 & 81.50 \\
\hline 22 & B2 & 24.86 & 75.14 \\
\hline 23 & B3 & 27.75 & 72.25 \\
\hline 24 & B4 & 38.15 & 61.85 \\
\hline 25 & B5 & 35.26 & 64.74 \\
\hline 26 & B6 & 39.31 & 60.69 \\
\hline 27 & B7 & 63.01 & 36.99 \\
\hline 28 & $\mathrm{~B} 8$ & 65.32 & 34.68 \\
\hline 29 & B9 & 82.66 & 17.34 \\
\hline 31 & $\mathrm{C} 1$ & 87.28 & 12.72 \\
\hline 33 & D1 & 87.28 & 12.72 \\
\hline 34 & D2 & 87.28 & 12.72 \\
\hline 35 & D3 & 87.28 & 12.72 \\
\hline 36 & D4 & 87.28 & 12.72 \\
\hline 37 & D5 & 87.28 & 12.72 \\
\hline 38 & D6 & 87.28 & 12.72 \\
\hline 40 & E1 & 82.08 & 17.92 \\
\hline 41 & E2 & 82.08 & 17.92 \\
\hline 42 & E3 & 82.08 & 17.92 \\
\hline 43 & E4 & 82.08 & 17.92 \\
\hline 44 & E5 & 84.97 & 15.03 \\
\hline 45 & E6 & 88.44 & 11.56 \\
\hline 50 & $\mathrm{~F} 1$ & 76.88 & 23.12 \\
\hline 51 & $\mathrm{~F} 2$ & 76.88 & 23.12 \\
\hline
\end{tabular}




\begin{tabular}{|c|c|c|c|}
\hline 52 & F3 & 76.88 & 23.12 \\
\hline 53 & $\mathrm{~F} 4$ & 82.66 & 17.34 \\
\hline 54 & F5 & 82.66 & 17.34 \\
\hline 55 & F6 & 82.66 & 17.34 \\
\hline 60 & G1 & 24.86 & 75.14 \\
\hline 61 & $\mathrm{G} 2$ & 76.88 & 23.12 \\
\hline 62 & G3 & 76.88 & 23.12 \\
\hline 63 & G4 & 76.88 & 23.12 \\
\hline 64 & G5 & 76.88 & 23.12 \\
\hline 65 & G6 & 82.66 & 17.34 \\
\hline 66 & $\mathrm{H} 1$ & 90.75 & 9.25 \\
\hline 67 & $\mathrm{H} 2$ & 96.53 & 3.47 \\
\hline 71 & I1 & 90.17 & 9.83 \\
\hline 72 & $\mathrm{I} 2$ & 96.53 & 3.47 \\
\hline 73 & $\mathrm{~J} 1$ & 93.64 & 6.36 \\
\hline 74 & $\mathrm{~J} 2$ & 96.53 & 3.47 \\
\hline 75 & $\mathrm{~K} 1$ & 38.73 & 61.27 \\
\hline 76 & $\mathrm{~K} 2$ & 38.73 & 61.27 \\
\hline 77 & K3 & 38.73 & 61.27 \\
\hline 78 & K4 & 53.18 & 46.82 \\
\hline 79 & K5 & 73.99 & 26.01 \\
\hline 80 & K6 & 73.99 & 26.01 \\
\hline 81 & K7 & 85.55 & 14.45 \\
\hline 90 & L1 & 92.49 & 7.51 \\
\hline 91 & M1 & 97.69 & 2.31 \\
\hline 92 & N1 & 94.22 & 5.78 \\
\hline 93 & $\mathrm{O} 1$ & 91.33 & 8.67 \\
\hline 94 & $\mathrm{O} 2$ & 91.33 & 8.67 \\
\hline 97 & $\mathrm{P} 1$ & 82.66 & 17.34 \\
\hline 98 & $\mathrm{P} 2$ & 82.66 & 17.34 \\
\hline 102 & Q1 & 82.66 & 17.34 \\
\hline 106 & $\mathrm{R} 1$ & 91.33 & 8.67 \\
\hline 108 & $\mathrm{~S} 1$ & 97.11 & 2.89 \\
\hline
\end{tabular}




\section{Appendix IV: Data Files for Case Study 3}

\begin{tabular}{|c|c|c|}
\hline & Table AIV.1: Ship Repair Task Description & \\
\hline Task & Description & Pred. \\
\hline & Treatment and Painting & \\
\hline 1 & install scuppers pipes (top side) & \\
\hline 2 & high pressure water (top side) & 1 \\
\hline 3 & treatment (top side) & 2 \\
\hline 4 & painting (top side) & 3 \\
\hline 5 & hull marks+name+ register port (top side) & 4 \\
\hline 6 & remove scuppers pipes (top side) & 5 \\
\hline 7 & scraping (vertical bottom) & \\
\hline 8 & high pressure water (vertical bottom) & 7 \\
\hline 9 & treatment (vertical bottom) & 8 \\
\hline 10 & painting (vertical bottom) & 9 \\
\hline 11 & hull marks (vertical bottom) & 10 \\
\hline 12 & protection speed - log/ echo sounder (flat bottom) & \\
\hline 13 & high pressure water (flat bottom) & 12 \\
\hline 14 & treatment (flat bottom) & 13 \\
\hline 15 & painting (flat bottom) & 14 \\
\hline 16 & hull marks (flat bottom) & 15 \\
\hline 17 & mount stages (moon pool) & \\
\hline 18 & high pressure water (moon pool) & 17 \\
\hline 19 & treatment (moon pool) & 18 \\
\hline 20 & painting (moon pool) & 19 \\
\hline 21 & change zinc anodes (moon pool) & 18 \\
\hline 22 & mount stages (sea chests and box coolers) & \\
\hline 23 & open grids (sea chests and box coolers) & \\
\hline 24 & install temporary lights 24 volts (sea chests and box coolers) & \\
\hline 25 & clean (sea chests and box coolers) & \\
\hline 26 & high pressure water (sea chests and box coolers) & \\
\hline 27 & treatment and paint (sea chests and box coolers) & 26 \\
\hline 28 & change zinc anodes (sea chests and box coolers) & 27 \\
\hline 29 & paint (sea chests and box coolers) & 27 \\
\hline 30 & remove temporary lights 24 volts (sea chests and box coolers) & 29 \\
\hline 31 & close grids (sea chests and box coolers) & 29 \\
\hline 32 & dismount stages (sea chests and box coolers) & 29 \\
\hline 33 & mount stages (tunnel thruster / azimuth retractable) & \\
\hline 34 & open grids (tunnel thruster / azimuth retractable) & \\
\hline 35 & high pressure water (tunnel thruster / azimuth retractable) & 33,34 \\
\hline 36 & treatment (tunnel thruster / azimuth retractable) & 35 \\
\hline
\end{tabular}




\begin{tabular}{|c|c|c|}
\hline 37 & paint (tunnel thruster / azimuth retractable) & 36 \\
\hline 38 & change zinc anodes (tunnel thruster / azimuth retractable) & 36 \\
\hline 39 & close grids (tunnel thruster / azimuth retractable) & 37,38 \\
\hline 40 & dismount stages (tunnel thruster / azimuth retractable) & 39 \\
\hline 41 & mount stages (moon pool internal and external parts) & \\
\hline 42 & scraping (moon pool internal and external parts) & 41 \\
\hline 43 & hight pressure water (moon pool internal and external parts) & 41 \\
\hline 44 & treat (moon pool internal and external parts) & 43 \\
\hline 45 & paint (moon pool internal and external parts) & 44 \\
\hline 46 & dismount stages (moon pool internal and external parts) & 45 \\
\hline 47 & change zinc anodes (moon pool internal and external parts) & 44 \\
\hline 48 & mount stages (hipap trucking) & \\
\hline 49 & scraping (hipap trucking) & 48 \\
\hline 50 & high pressure water (hipap trucking) & 49 \\
\hline 51 & treat (hipap trucking) & 50 \\
\hline 52 & paint (hipap trucking) & 51 \\
\hline \multirow[t]{2}{*}{53} & dismount stages (hipap trucking) & 52 \\
\hline & Steel work & \\
\hline 54 & remove (sacrifice anodes) & \\
\hline 55 & mount / weld (sacrifice anodes) & 54 \\
\hline 56 & mount stages (after body repair) & \\
\hline 57 & mark (after body repair) & 56 \\
\hline 58 & cut (after body repair) & 57 \\
\hline 59 & prefabricate (after body repair) & 58 \\
\hline 60 & mounting (after body repair) & 59 \\
\hline 61 & welding (after body repair) & 60 \\
\hline 62 & survey inspection (after body repair) & 61 \\
\hline 63 & ultrasonic and vacuum test (after body repair) & 62 \\
\hline 64 & treatment and paint (after body repair) & 63 \\
\hline \multirow[t]{2}{*}{65} & remove stages (after body repair) & 64 \\
\hline & Valves & \\
\hline 66 & remove from board (sea valves and overboard valves) & \\
\hline 67 & transport to workshop (sea valves and overboard valves) & 66 \\
\hline 68 & open valves on workshop (sea valves and overboard valves) & 66 \\
\hline 69 & repair (sea valves and overboard valves) & 68 \\
\hline 70 & test/ inspection (sea valves and overboard valves) & 69 \\
\hline 71 & transport to board (sea valves and overboard valves) & 70 \\
\hline 72 & mounting on board (sea valves and overboard valves) & 71 \\
\hline 73 & remove from board (hipap valves and gate valves) & \\
\hline 74 & transport to workshop (hipap valves and gate valves) & 73 \\
\hline 75 & open valves (hipap valves and gate valves) & 74 \\
\hline 76 & repair (hipap valves and gate valves) & 75 \\
\hline
\end{tabular}




\begin{tabular}{|c|c|c|}
\hline 77 & test/ inspection (hipap valves and gate valves) & 76 \\
\hline 78 & transport to board (hipap valves and gate valves) & 77 \\
\hline \multirow[t]{2}{*}{79} & mounting (hipap valves and gate valves) & 78 \\
\hline & bottom plugs & \\
\hline 80 & remove (bottom plugs) & \\
\hline 81 & replaced (bottom plugs) & 80 \\
\hline \multirow[t]{2}{*}{82} & vacuum test (bottom plugs) & 80 \\
\hline & Propeller & \\
\hline 83 & mount stages (propeller) & \\
\hline 84 & clean/ polish propeller (propeller) & 83 \\
\hline 85 & install protection for painting (propeller) & 84 \\
\hline 86 & remove protection (propeller) & 85 \\
\hline \multirow[t]{2}{*}{87} & dismount stages (propeller) & 86 \\
\hline & Tank cleaning & \\
\hline 88 & clean tank \#1 (diesel tanks) & \\
\hline \multirow[t]{2}{*}{89} & clean tank \#2 (fresh water tanks) & \\
\hline & Support & \\
\hline 90 & mount stages (n/a) & \\
\hline 91 & weld eyes pad (n/a) & \\
\hline 92 & remove eyes pad (n/a) & 90.91 \\
\hline \multirow[t]{2}{*}{93} & dismount stages (n/a) & 90.91 \\
\hline & Pipes & \\
\hline 94 & cut (bio guard antifouling system) & \\
\hline 95 & fabricate (bio guard antifouling system) & 94 \\
\hline 96 & mounting (bio guard antifouling system) & 95 \\
\hline 97 & inspection (bio guard antifouling system) & 96 \\
\hline \multirow[t]{2}{*}{98} & test (bio guard antifouling system) & 97 \\
\hline & treatment and painting & \\
\hline 99 & mount stages (deck crane) & \\
\hline 100 & degreasing (deck crane) & 99 \\
\hline 101 & treatment st 3 (deck crane) & 100 \\
\hline 102 & painting (deck crane) & 101 \\
\hline \multirow[t]{2}{*}{103} & dismount stages (deck crane) & 102 \\
\hline & Support & \\
\hline 104 & mount stages hydraulics hoses (deck crane) & \\
\hline \multirow[t]{2}{*}{105} & dismounting stages hydraulics hoses (deck crane) & 104 \\
\hline & life boats & \\
\hline 106 & prepare blocks for receive boat on shipyard-min. $300 \mathrm{~mm}$ of height (life boats) & \\
\hline 107 & mounting stages for access forward and after locations hooks (life boats) & \\
\hline 108 & supply equipment for lifting $500 \mathrm{~kg}$ height min. 06 meters (life boats) & \\
\hline
\end{tabular}


Table AIV.2: Task-Modes-Resource Requirements

\begin{tabular}{|c|c|c|c|}
\hline Task & Mode & Qty. & Description \\
\hline 1 & 1 & 2 & carrier \\
\hline 1 & 1 & 2 & plumber \\
\hline 1 & 2 & 1 & plumber \\
\hline 2 & 1 & 2 & fluid jet \\
\hline 2 & 1 & 2 & support \\
\hline 2 & 2 & 1 & fluid jet \\
\hline 3 & 1 & 2 & painter \\
\hline 3 & 2 & 1 & painter \\
\hline 4 & 1 & 2 & support \\
\hline 4 & 1 & 4 & painter \\
\hline 4 & 2 & 4 & painter \\
\hline 4 & 2 & 2 & support \\
\hline 5 & 1 & 1 & support \\
\hline 5 & 2 & 1 & support \\
\hline 6 & 1 & 1 & plumber \\
\hline 6 & 2 & 1 & carrier \\
\hline 7 & 1 & 2 & fluid jet \\
\hline 7 & 2 & 1 & fluid jet \\
\hline 8 & 1 & 2 & support \\
\hline 8 & 1 & 2 & fluid jet \\
\hline 8 & 2 & 1 & fluid jet \\
\hline 9 & 1 & 2 & painter \\
\hline 9 & 2 & 1 & painter \\
\hline 10 & 1 & 2 & support \\
\hline 10 & 1 & 4 & painter \\
\hline 10 & 2 & 4 & painter \\
\hline 11 & 1 & 1 & support \\
\hline 11 & 2 & 1 & support \\
\hline 12 & 1 & 1 & support \\
\hline 12 & 2 & 1 & support \\
\hline 13 & 1 & 2 & support \\
\hline 13 & 1 & 2 & fluid jet \\
\hline 13 & 2 & 1 & fluid jet \\
\hline 14 & 1 & 2 & painter \\
\hline 14 & 2 & 1 & painter \\
\hline 15 & 1 & 2 & support \\
\hline 15 & 1 & 4 & painter \\
\hline 15 & 2 & 4 & painter \\
\hline 16 & 1 & 1 & painter \\
\hline 16 & 2 & 1 & support \\
\hline 17 & 1 & 1 & assembler \\
\hline 17 & 2 & 2 & assembler \\
\hline
\end{tabular}




\begin{tabular}{|c|c|c|c|}
\hline 18 & 1 & 2 & support \\
\hline 18 & 1 & 2 & fluid jet \\
\hline 18 & 2 & 1 & fluid jet \\
\hline 19 & 1 & 2 & painter \\
\hline 19 & 2 & 1 & painter \\
\hline 20 & 1 & 5 & painter \\
\hline 20 & 1 & 2 & support \\
\hline 20 & 2 & 4 & painter \\
\hline 21 & 1 & 1 & assembler \\
\hline 21 & 1 & 2 & welder \\
\hline 21 & 1 & 1 & blow torched \\
\hline 21 & 1 & 1 & painter \\
\hline 21 & 2 & 1 & blow torched \\
\hline 21 & 2 & 1 & assembler \\
\hline 21 & 2 & 1 & welder \\
\hline 21 & 2 & 1 & painter \\
\hline 22 & 1 & 3 & assembler \\
\hline 22 & 2 & 3 & assembler \\
\hline 23 & 1 & 2 & mechanic \\
\hline 23 & 2 & 1 & mechanic \\
\hline 24 & 1 & 1 & electrician \\
\hline 24 & 2 & 1 & electrician \\
\hline 25 & 1 & 10 & fluid jet \\
\hline 26 & 1 & 10 & fluid jet \\
\hline 27 & 1 & 10 & painter \\
\hline 27 & 1 & 10 & support \\
\hline 28 & 1 & 1 & painter \\
\hline 28 & 1 & 2 & welder \\
\hline 28 & 1 & 1 & assembler \\
\hline 28 & 1 & 1 & blow torched \\
\hline 28 & 2 & 1 & assembler \\
\hline 28 & 2 & 1 & welder \\
\hline 28 & 2 & 1 & blow torched \\
\hline 28 & 2 & 1 & painter \\
\hline 29 & 1 & 10 & painter \\
\hline 29 & 2 & 10 & painter \\
\hline 30 & 1 & 1 & electrician \\
\hline 31 & 1 & 2 & mechanic \\
\hline 32 & 1 & 3 & assembler \\
\hline 33 & 1 & 3 & assembler \\
\hline 34 & 1 & 4 & mechanic \\
\hline 35 & 1 & 20 & fluid jet \\
\hline 36 & 1 & 10 & painter \\
\hline 37 & 1 & 10 & painter \\
\hline
\end{tabular}




\begin{tabular}{|c|c|c|c|}
\hline 38 & 1 & 1 & assembler \\
\hline 38 & 1 & 1 & painter \\
\hline 38 & 1 & 1 & blow torched \\
\hline 38 & 1 & 2 & welder \\
\hline 38 & 2 & 1 & welder \\
\hline 38 & 2 & 1 & blow torched \\
\hline 38 & 2 & 1 & assembler \\
\hline 38 & 2 & 1 & painter \\
\hline 39 & 1 & 2 & mechanic \\
\hline 40 & 1 & 3 & assembler \\
\hline 41 & 1 & 3 & assembler \\
\hline 42 & 1 & 2 & fluid jet \\
\hline 43 & 1 & 20 & fluid jet \\
\hline 44 & 1 & 10 & painter \\
\hline 45 & 1 & 10 & painter \\
\hline 46 & 1 & 3 & assembler \\
\hline 47 & 1 & 2 & welder \\
\hline 47 & 1 & 1 & blow torched \\
\hline 47 & 1 & 1 & assembler \\
\hline 47 & 1 & 1 & painter \\
\hline 48 & 1 & 9 & assembler \\
\hline 49 & 1 & 4 & fluid jet \\
\hline 50 & 1 & 20 & fluid jet \\
\hline 51 & 1 & 10 & painter \\
\hline 52 & 1 & 10 & painter \\
\hline 53 & 1 & 6 & assembler \\
\hline 54 & 1 & 5 & welder \\
\hline 54 & 1 & 5 & support \\
\hline 54 & 1 & 5 & blow torched \\
\hline 54 & 1 & 5 & assembler \\
\hline 54 & 1 & 5 & painter \\
\hline 55 & 1 & 5 & welder \\
\hline 55 & 1 & 2 & support \\
\hline 56 & 1 & 9 & assembler scaffolding \\
\hline 57 & 1 & 1 & support \\
\hline 58 & 1 & 5 & blow torched \\
\hline 59 & 1 & 10 & brazier \\
\hline 59 & 1 & 10 & welder \\
\hline 60 & 1 & 10 & assembler \\
\hline 61 & 1 & 20 & welder \\
\hline 62 & 1 & 1 & quality control \\
\hline 63 & 1 & 1 & quality control \\
\hline 64 & 1 & 10 & support \\
\hline 64 & 1 & 10 & painter \\
\hline
\end{tabular}




\begin{tabular}{|c|c|c|c|}
\hline 65 & 1 & 6 & assembler \\
\hline 66 & 1 & 10 & plumber \\
\hline 67 & 1 & 1 & plumber \\
\hline 67 & 1 & 10 & carrier \\
\hline 67 & 1 & 1 & Truck 1 \\
\hline 68 & 1 & 1 & welder \\
\hline 68 & 1 & 1 & Valves Repairing \\
\hline 69 & 1 & 1 & welder \\
\hline 69 & 1 & 1 & Valves Repairing \\
\hline 70 & 1 & 1 & welder \\
\hline 70 & 1 & 1 & Valves Repairing \\
\hline 71 & 1 & 10 & carrier \\
\hline 72 & 1 & 10 & plumber \\
\hline 73 & 1 & 10 & plumber \\
\hline 74 & 1 & 1 & plumber \\
\hline 74 & 1 & 1 & Truck 1 \\
\hline 74 & 1 & 10 & carrier \\
\hline 75 & 1 & 1 & Valves Repairing \\
\hline 75 & 1 & 1 & welder \\
\hline 76 & 1 & 1 & Valves Repairing \\
\hline 76 & 1 & 1 & welder \\
\hline 77 & 1 & 1 & Valves Repairing \\
\hline 77 & 1 & 1 & welder \\
\hline 78 & 1 & 10 & carrier \\
\hline 79 & 1 & 10 & plumber \\
\hline 80 & 1 & 4 & support \\
\hline 80 & 1 & 4 & dock aid \\
\hline 81 & 1 & 4 & support \\
\hline 81 & 1 & 4 & dock aid \\
\hline 82 & 1 & 4 & support \\
\hline 82 & 1 & 4 & dock aid \\
\hline 83 & 1 & 10 & assembler scaffolding \\
\hline 84 & 1 & 10 & fluid jet \\
\hline 85 & 1 & 10 & painter \\
\hline 86 & 1 & 10 & fluid jet \\
\hline 87 & 1 & 10 & assembler scaffolding \\
\hline 88 & 1 & 20 & fluid jet \\
\hline 88 & 1 & 20 & safety \\
\hline 89 & 1 & 20 & fluid jet \\
\hline 89 & 1 & 20 & safety \\
\hline 90 & 1 & 10 & support \\
\hline 91 & 1 & 10 & support \\
\hline 92 & 1 & 10 & support \\
\hline 93 & 1 & 10 & support \\
\hline
\end{tabular}




\begin{tabular}{|c|c|c|c|}
\hline 94 & 1 & 1 & Pipe Bending \\
\hline 94 & 1 & 1 & fluid jet \\
\hline 95 & 1 & 1 & Pipe Bending \\
\hline 95 & 1 & 1 & fluid jet \\
\hline 96 & 1 & 1 & Pipe Bending \\
\hline 96 & 1 & 1 & fluid jet \\
\hline 97 & 1 & 1 & Pipe Bending \\
\hline 97 & 1 & 1 & fluid jet \\
\hline 98 & 1 & 1 & Pipe Bending \\
\hline 98 & 1 & 1 & fluid jet \\
\hline 99 & 1 & 1 & crane operator \\
\hline 99 & 1 & 5 & painter \\
\hline 99 & 1 & 5 & fluid jet \\
\hline 100 & 1 & 5 & fluid jet \\
\hline 100 & 1 & 1 & crane operator \\
\hline 100 & 1 & 5 & painter \\
\hline 101 & 1 & 5 & fluid jet \\
\hline 101 & 1 & 1 & crane operator \\
\hline 101 & 1 & 5 & painter \\
\hline 102 & 1 & 1 & crane operator \\
\hline 102 & 1 & 5 & fluid jet \\
\hline 102 & 1 & 5 & painter \\
\hline 103 & 1 & 5 & painter \\
\hline 103 & 1 & 1 & crane operator \\
\hline 103 & 1 & 5 & fluid jet \\
\hline 104 & 1 & 1 & support \\
\hline 105 & 1 & 1 & support \\
\hline 106 & 1 & 1 & support \\
\hline 107 & 1 & 2 & assembler scaffolding \\
\hline 108 & 1 & 1 & Pulley 1 \\
\hline 108 & 1 & 1 & support \\
\hline 108 & 1 & 1 & machine operator \\
\hline 109 & 1 & 1 & Power Generator 1 \\
\hline 109 & 1 & 1 & support \\
\hline 110 & 1 & 5 & support \\
\hline 111 & 1 & 5 & support \\
\hline
\end{tabular}




\begin{tabular}{|c|c|c|}
\hline & Table AIV.3: Multi-skilled resources & \\
\hline Resource & Description & Rank \\
\hline R76 & plumber & 1 \\
\hline R76 & quality control & 2 \\
\hline R76 & safety & 3 \\
\hline R77 & machine operator & 1 \\
\hline R77 & electrician & 2 \\
\hline R77 & welder & 3 \\
\hline $\mathrm{R} 78$ & fluid jet & 1 \\
\hline R78 & electrician & 2 \\
\hline R78 & plumber & 3 \\
\hline R79 & blow torched & 1 \\
\hline R79 & dock aid & 2 \\
\hline R79 & carrier & 3 \\
\hline $\mathrm{R} 80$ & assembler scaffolding & 1 \\
\hline $\mathrm{R} 80$ & safety & 2 \\
\hline $\mathrm{R} 81$ & general services & 1 \\
\hline $\mathrm{R} 81$ & machine operator & 2 \\
\hline $\mathrm{R} 81$ & quality control & 3 \\
\hline $\mathrm{R} 82$ & carrier & 1 \\
\hline $\mathrm{R} 82$ & mechanic & 2 \\
\hline $\mathrm{R} 82$ & assembler scaffolding & 3 \\
\hline $\mathrm{R} 83$ & support & 1 \\
\hline $\mathrm{R} 83$ & blow torched & 3 \\
\hline $\mathrm{R} 84$ & painter & 1 \\
\hline $\mathrm{R} 84$ & assembler & 2 \\
\hline R84 & electrician & 3 \\
\hline $\mathrm{R} 85$ & welder & 1 \\
\hline $\mathrm{R} 85$ & machine operator & 2 \\
\hline R86 & safety & 1 \\
\hline R86 & painter & 2 \\
\hline R86 & general services & 3 \\
\hline $\mathrm{R} 87$ & fluid jet & 1 \\
\hline $\mathrm{R} 87$ & crane operator & 2 \\
\hline R87 & fireman & 3 \\
\hline $\mathrm{R} 88$ & carpenter & 1 \\
\hline $\mathrm{R} 88$ & general services & 2 \\
\hline $\mathrm{R} 88$ & crane operator & 3 \\
\hline R89 & carpenter & 1 \\
\hline R89 & brazier & 2 \\
\hline R89 & fireman & 3 \\
\hline R90 & machine operator & 1 \\
\hline R90 & welder & 2 \\
\hline R90 & assembler scaffolding & 3 \\
\hline
\end{tabular}




\begin{tabular}{|c|c|c|}
\hline R91 & support & 1 \\
\hline R91 & assembler & 3 \\
\hline R92 & fireman & 1 \\
\hline R92 & carrier & 2 \\
\hline R92 & brazier & 3 \\
\hline R93 & crane operator & 1 \\
\hline R93 & general services & 2 \\
\hline R93 & fluid jet & 3 \\
\hline R94 & machine operator & 1 \\
\hline R94 & general services & 2 \\
\hline R94 & carrier & 3 \\
\hline R95 & welder & 1 \\
\hline R95 & fireman & 2 \\
\hline R95 & assembler scaffolding & 3 \\
\hline R96 & dock aid & 1 \\
\hline R96 & support & 2 \\
\hline R96 & electrician & 3 \\
\hline R97 & crane operator & 1 \\
\hline R97 & plumber & 2 \\
\hline R97 & carpenter & 3 \\
\hline R98 & electrician & 1 \\
\hline R98 & plumber & 2 \\
\hline R98 & brazier & 3 \\
\hline R99 & carrier & 1 \\
\hline R99 & fluid jet & 2 \\
\hline R99 & fireman & 3 \\
\hline R100 & brazier & 1 \\
\hline R100 & carrier & 2 \\
\hline R100 & fireman & 3 \\
\hline R101 & safety & 1 \\
\hline R101 & crane operator & 2 \\
\hline R101 & assembler & 3 \\
\hline R102 & assembler & 1 \\
\hline R102 & safety & 2 \\
\hline R102 & carrier & 3 \\
\hline R103 & blow torched & 1 \\
\hline R103 & brazier & 2 \\
\hline R103 & plumber & 3 \\
\hline R104 & brazier & 1 \\
\hline R104 & quality control & 2 \\
\hline R104 & safety & 3 \\
\hline R105 & painter & 1 \\
\hline R105 & mechanic & 2 \\
\hline R105 & machine operator & 3 \\
\hline
\end{tabular}




\begin{tabular}{|c|c|c|}
\hline R106 & quality control & 1 \\
\hline R106 & machine operator & 2 \\
\hline R106 & welder & 3 \\
\hline R107 & quality control & 1 \\
\hline R107 & welder & 2 \\
\hline R108 & dock aid & 1 \\
\hline R108 & fireman & 2 \\
\hline R108 & carpenter & 3 \\
\hline R109 & carrier & 1 \\
\hline R109 & dock aid & 2 \\
\hline R109 & safety & 3 \\
\hline R110 & carpenter & 1 \\
\hline R110 & general services & 2 \\
\hline R110 & plumber & 3 \\
\hline R111 & mechanic & 1 \\
\hline R111 & dock aid & 2 \\
\hline R111 & assembler & 3 \\
\hline R112 & general services & 1 \\
\hline R112 & fireman & 2 \\
\hline R112 & assembler scaffolding & 3 \\
\hline R113 & dock aid & 1 \\
\hline R113 & electrician & 2 \\
\hline R113 & painter & 3 \\
\hline R114 & general services & 1 \\
\hline R114 & fluid jet & 2 \\
\hline R114 & crane operator & 3 \\
\hline R115 & fireman & 1 \\
\hline R115 & brazier & 2 \\
\hline R116 & welder & 1 \\
\hline R116 & safety & 3 \\
\hline R117 & fireman & 1 \\
\hline R117 & blow torched & 2 \\
\hline R117 & plumber & 3 \\
\hline R118 & dock aid & 1 \\
\hline R118 & electrician & 2 \\
\hline R118 & general services & 3 \\
\hline R119 & brazier & 1 \\
\hline R119 & quality control & 2 \\
\hline R119 & carpenter & 3 \\
\hline R120 & mechanic & 1 \\
\hline R120 & safety & 2 \\
\hline R120 & assembler scaffolding & 3 \\
\hline R121 & carpenter & 1 \\
\hline R121 & brazier & 2 \\
\hline
\end{tabular}




\begin{tabular}{|c|c|c|}
\hline R121 & welder & 3 \\
\hline R122 & safety & 1 \\
\hline R122 & mechanic & 2 \\
\hline R122 & quality control & 3 \\
\hline R123 & assembler & 1 \\
\hline R123 & blow torched & 3 \\
\hline R124 & plumber & 1 \\
\hline R124 & brazier & 2 \\
\hline R124 & carpenter & 3 \\
\hline $\mathrm{R} 125$ & machine operator & 1 \\
\hline $\mathrm{R} 125$ & carpenter & 2 \\
\hline R125 & mechanic & 3 \\
\hline R126 & brazier & 1 \\
\hline R126 & blow torched & 2 \\
\hline R126 & dock aid & 3 \\
\hline R127 & machine operator & 1 \\
\hline R127 & carrier & 2 \\
\hline $\mathrm{R} 127$ & painter & 3 \\
\hline R128 & welder & 1 \\
\hline R128 & plumber & 2 \\
\hline R128 & safety & 3 \\
\hline R129 & brazier & 1 \\
\hline R129 & assembler & 2 \\
\hline R129 & quality control & 3 \\
\hline R130 & general services & 1 \\
\hline $\mathrm{R} 130$ & dock aid & 2 \\
\hline $\mathrm{R} 130$ & brazier & 3 \\
\hline R131 & crane operator & 1 \\
\hline R131 & plumber & 2 \\
\hline R131 & brazier & 3 \\
\hline R132 & mechanic & 1 \\
\hline R132 & dock aid & 2 \\
\hline R132 & plumber & 3 \\
\hline R133 & assembler scaffolding & 1 \\
\hline R133 & dock aid & 2 \\
\hline R134 & general services & 1 \\
\hline R134 & blow torched & 2 \\
\hline R134 & safety & 3 \\
\hline R135 & fireman & 1 \\
\hline $\mathrm{R} 135$ & machine operator & 2 \\
\hline R135 & carrier & 3 \\
\hline $\mathrm{R} 136$ & dock aid & 1 \\
\hline R136 & machine operator & 2 \\
\hline R136 & support & 3 \\
\hline
\end{tabular}




\begin{tabular}{|c|c|c|}
\hline R137 & crane operator & 1 \\
\hline R137 & painter & 2 \\
\hline R137 & electrician & 3 \\
\hline $\mathrm{R} 138$ & quality control & 1 \\
\hline R138 & plumber & 2 \\
\hline R138 & assembler scaffolding & 3 \\
\hline R139 & mechanic & 1 \\
\hline R139 & safety & 3 \\
\hline R140 & blow torched & 1 \\
\hline $\mathrm{R} 140$ & assembler & 2 \\
\hline R140 & welder & 3 \\
\hline R141 & carrier & 1 \\
\hline R141 & safety & 2 \\
\hline R141 & assembler scaffolding & 3 \\
\hline R142 & carpenter & 1 \\
\hline R142 & blow torched & 2 \\
\hline R142 & machine operator & 3 \\
\hline R143 & painter & 1 \\
\hline R143 & safety & 2 \\
\hline R143 & blow torched & 3 \\
\hline R144 & general services & 1 \\
\hline R144 & brazier & 2 \\
\hline R144 & machine operator & 3 \\
\hline $\mathrm{R} 145$ & quality control & 1 \\
\hline $\mathrm{R} 145$ & general services & 3 \\
\hline $\mathrm{R} 146$ & fireman & 1 \\
\hline R146 & carpenter & 2 \\
\hline R146 & assembler & 3 \\
\hline R147 & dock aid & 1 \\
\hline R147 & painter & 2 \\
\hline R147 & carpenter & 3 \\
\hline R148 & safety & 1 \\
\hline R148 & quality control & 2 \\
\hline R148 & support & 3 \\
\hline R149 & support & 1 \\
\hline R149 & carrier & 2 \\
\hline R149 & assembler & 3 \\
\hline $\mathrm{R} 150$ & mechanic & 1 \\
\hline R150 & fireman & 2 \\
\hline $\mathrm{R} 150$ & quality control & 3 \\
\hline R151 & carrier & 1 \\
\hline $\mathrm{R} 151$ & safety & 2 \\
\hline $\mathrm{R} 151$ & mechanic & 3 \\
\hline $\mathrm{R} 152$ & crane operator & 1 \\
\hline
\end{tabular}




\begin{tabular}{|c|c|c|}
\hline $\mathrm{R} 152$ & general services & 2 \\
\hline R152 & machine operator & 3 \\
\hline R153 & quality control & 1 \\
\hline $\mathrm{R} 153$ & machine operator & 2 \\
\hline R153 & general services & 3 \\
\hline $\mathrm{R} 154$ & assembler scaffolding & 1 \\
\hline R154 & machine operator & 2 \\
\hline $\mathrm{R} 154$ & quality control & 3 \\
\hline $\mathrm{R} 155$ & machine operator & 1 \\
\hline $\mathrm{R} 155$ & mechanic & 2 \\
\hline $\mathrm{R} 155$ & fireman & 3 \\
\hline R156 & crane operator & 1 \\
\hline R156 & fluid jet & 2 \\
\hline $\mathrm{R} 156$ & electrician & 3 \\
\hline $\mathrm{R} 157$ & mechanic & 1 \\
\hline $\mathrm{R} 157$ & welder & 2 \\
\hline R157 & fluid jet & 3 \\
\hline $\mathrm{R} 158$ & assembler & 1 \\
\hline $\mathrm{R} 158$ & general services & 2 \\
\hline $\mathrm{R} 158$ & crane operator & 3 \\
\hline R159 & assembler & 1 \\
\hline R159 & blow torched & 2 \\
\hline R159 & plumber & 3 \\
\hline R160 & assembler & 1 \\
\hline R160 & dock aid & 2 \\
\hline $\mathrm{R} 160$ & blow torched & 3 \\
\hline R161 & fluid jet & 1 \\
\hline R161 & painter & 2 \\
\hline R161 & machine operator & 3 \\
\hline R162 & welder & 1 \\
\hline R162 & general services & 2 \\
\hline R162 & blow torched & 3 \\
\hline R163 & assembler scaffolding & 1 \\
\hline R163 & brazier & 2 \\
\hline R163 & plumber & 3 \\
\hline R164 & crane operator & 1 \\
\hline R164 & quality control & 2 \\
\hline R164 & electrician & 3 \\
\hline R165 & assembler scaffolding & 1 \\
\hline R165 & quality control & 2 \\
\hline R165 & fireman & 3 \\
\hline R166 & blow torched & 1 \\
\hline R166 & electrician & 2 \\
\hline R166 & fluid jet & 3 \\
\hline
\end{tabular}




\begin{tabular}{|c|c|c|}
\hline R167 & crane operator & 1 \\
\hline R167 & blow torched & 2 \\
\hline R167 & painter & 3 \\
\hline R168 & safety & 1 \\
\hline R168 & assembler & 2 \\
\hline R168 & painter & 3 \\
\hline R169 & support & 1 \\
\hline R169 & dock aid & 2 \\
\hline R169 & assembler scaffolding & 3 \\
\hline R170 & blow torched & 1 \\
\hline $\mathrm{R} 170$ & dock aid & 2 \\
\hline $\mathrm{R} 170$ & general services & 3 \\
\hline R171 & plumber & 1 \\
\hline R171 & carpenter & 2 \\
\hline R171 & painter & 3 \\
\hline R172 & carrier & 1 \\
\hline R172 & quality control & 2 \\
\hline $\mathrm{R} 172$ & fireman & 3 \\
\hline R173 & painter & 1 \\
\hline R173 & crane operator & 2 \\
\hline R173 & support & 3 \\
\hline $\mathrm{R} 174$ & blow torched & 1 \\
\hline R174 & fluid jet & 2 \\
\hline $\mathrm{R} 174$ & dock aid & 3 \\
\hline R175 & machine operator & 1 \\
\hline $\mathrm{R} 175$ & assembler scaffolding & 2 \\
\hline R175 & general services & 3 \\
\hline R176 & fireman & 1 \\
\hline R176 & carpenter & 2 \\
\hline R177 & plumber & 1 \\
\hline R177 & safety & 2 \\
\hline R177 & painter & 3 \\
\hline R178 & fireman & 1 \\
\hline R178 & electrician & 2 \\
\hline R179 & painter & 1 \\
\hline R179 & assembler & 2 \\
\hline R179 & carpenter & 3 \\
\hline R180 & fluid jet & 1 \\
\hline R180 & crane operator & 2 \\
\hline $\mathrm{R} 180$ & general services & 3 \\
\hline $\mathrm{R} 181$ & carrier & 1 \\
\hline R181 & electrician & 2 \\
\hline R181 & general services & 3 \\
\hline R182 & mechanic & 1 \\
\hline
\end{tabular}




\begin{tabular}{|c|c|c|}
\hline R182 & machine operator & 2 \\
\hline R182 & assembler scaffolding & 3 \\
\hline R183 & painter & 1 \\
\hline $\mathrm{R} 183$ & welder & 2 \\
\hline R183 & crane operator & 3 \\
\hline R184 & safety & 1 \\
\hline R184 & electrician & 2 \\
\hline $\mathrm{R} 184$ & assembler & 3 \\
\hline $\mathrm{R} 185$ & assembler scaffolding & 1 \\
\hline $\mathrm{R} 185$ & fluid jet & 2 \\
\hline $\mathrm{R} 185$ & support & 3 \\
\hline R186 & mechanic & 1 \\
\hline R186 & welder & 2 \\
\hline R186 & crane operator & 3 \\
\hline $\mathrm{R} 187$ & mechanic & 1 \\
\hline R187 & assembler & 2 \\
\hline R187 & safety & 3 \\
\hline $\mathrm{R} 188$ & plumber & 1 \\
\hline R188 & blow torched & 2 \\
\hline R188 & electrician & 3 \\
\hline R189 & carrier & 1 \\
\hline R189 & assembler scaffolding & 2 \\
\hline R189 & brazier & 3 \\
\hline R190 & crane operator & 1 \\
\hline R190 & electrician & 2 \\
\hline R191 & electrician & 1 \\
\hline R191 & assembler & 2 \\
\hline R191 & crane operator & 3 \\
\hline R192 & crane operator & 1 \\
\hline R192 & brazier & 2 \\
\hline R193 & quality control & 1 \\
\hline R193 & carpenter & 2 \\
\hline R193 & dock aid & 3 \\
\hline R194 & quality control & 1 \\
\hline R194 & safety & 2 \\
\hline R194 & plumber & 3 \\
\hline R195 & dock aid & 1 \\
\hline R195 & plumber & 2 \\
\hline R195 & quality control & 3 \\
\hline R196 & painter & 1 \\
\hline R196 & fluid jet & 2 \\
\hline R196 & welder & 3 \\
\hline R197 & plumber & 1 \\
\hline R197 & brazier & 2 \\
\hline
\end{tabular}




\begin{tabular}{|c|c|c|}
\hline R197 & fluid jet & 3 \\
\hline R198 & mechanic & 1 \\
\hline R198 & support & 2 \\
\hline R198 & general services & 3 \\
\hline R199 & carrier & 1 \\
\hline R199 & assembler scaffolding & 2 \\
\hline R199 & machine operator & 3 \\
\hline R200 & fireman & 1 \\
\hline R200 & quality control & 2 \\
\hline R200 & brazier & 3 \\
\hline R201 & machine operator & 1 \\
\hline R201 & brazier & 2 \\
\hline R201 & carpenter & 3 \\
\hline R202 & electrician & 1 \\
\hline R202 & dock aid & 2 \\
\hline R202 & painter & 3 \\
\hline R203 & crane operator & 1 \\
\hline R203 & plumber & 2 \\
\hline R203 & carrier & 3 \\
\hline R204 & machine operator & 1 \\
\hline R205 & brazier & 1 \\
\hline R205 & carpenter & 2 \\
\hline R205 & carrier & 3 \\
\hline R206 & general services & 1 \\
\hline R206 & brazier & 3 \\
\hline R207 & electrician & 1 \\
\hline R207 & blow torched & 2 \\
\hline R207 & mechanic & 3 \\
\hline R208 & quality control & 1 \\
\hline R208 & general services & 2 \\
\hline R209 & assembler scaffolding & 1 \\
\hline R209 & general services & 2 \\
\hline R209 & carpenter & 3 \\
\hline $\mathrm{R} 210$ & blow torched & 1 \\
\hline $\mathrm{R} 210$ & brazier & 2 \\
\hline $\mathrm{R} 210$ & fireman & 3 \\
\hline $\mathrm{R} 211$ & crane operator & 1 \\
\hline R211 & brazier & 2 \\
\hline R211 & welder & 3 \\
\hline R212 & general services & 1 \\
\hline $\mathrm{R} 212$ & dock aid & 2 \\
\hline R212 & blow torched & 3 \\
\hline $\mathrm{R} 213$ & crane operator & 1 \\
\hline R213 & fireman & 2 \\
\hline
\end{tabular}




\begin{tabular}{|c|c|c|}
\hline R213 & quality control & 3 \\
\hline R214 & quality control & 1 \\
\hline $\mathrm{R} 214$ & carrier & 2 \\
\hline R214 & assembler scaffolding & 3 \\
\hline R215 & fireman & 1 \\
\hline $\mathrm{R} 215$ & assembler & 2 \\
\hline R215 & fluid jet & 3 \\
\hline $\mathrm{R} 216$ & carrier & 1 \\
\hline R216 & brazier & 2 \\
\hline R216 & fluid jet & 3 \\
\hline R217 & safety & 1 \\
\hline R217 & machine operator & 2 \\
\hline R217 & mechanic & 3 \\
\hline $\mathrm{R} 218$ & support & 1 \\
\hline R218 & blow torched & 2 \\
\hline R218 & assembler scaffolding & 3 \\
\hline R219 & plumber & 1 \\
\hline R219 & fireman & 2 \\
\hline $\mathrm{R} 220$ & painter & 1 \\
\hline $\mathrm{R} 220$ & dock aid & 2 \\
\hline $\mathrm{R} 220$ & plumber & 3 \\
\hline $\mathrm{R} 221$ & dock aid & 1 \\
\hline R221 & support & 2 \\
\hline R221 & fireman & 3 \\
\hline R222 & painter & 1 \\
\hline R222 & fluid jet & 2 \\
\hline R222 & electrician & 3 \\
\hline R223 & dock aid & 1 \\
\hline R223 & general services & 2 \\
\hline $\mathrm{R} 223$ & support & 3 \\
\hline R224 & welder & 1 \\
\hline R224 & blow torched & 2 \\
\hline $\mathrm{R} 225$ & dock aid & 1 \\
\hline $\mathrm{R} 225$ & assembler & 2 \\
\hline R226 & carpenter & 1 \\
\hline R226 & plumber & 2 \\
\hline R226 & brazier & 3 \\
\hline R227 & welder & 1 \\
\hline R227 & fluid jet & 2 \\
\hline R227 & carrier & 3 \\
\hline R228 & quality control & 1 \\
\hline R228 & assembler & 2 \\
\hline R228 & crane operator & 3 \\
\hline R229 & support & 1 \\
\hline
\end{tabular}




\begin{tabular}{|c|c|c|}
\hline R229 & assembler scaffolding & 2 \\
\hline R229 & blow torched & 3 \\
\hline R230 & quality control & 1 \\
\hline $\mathrm{R} 230$ & fireman & 2 \\
\hline $\mathrm{R} 230$ & assembler & 3 \\
\hline R231 & crane operator & 1 \\
\hline $\mathrm{R} 231$ & carrier & 3 \\
\hline R232 & painter & 1 \\
\hline R232 & assembler & 2 \\
\hline $\mathrm{R} 232$ & brazier & 3 \\
\hline R233 & mechanic & 1 \\
\hline R233 & assembler & 2 \\
\hline R233 & fireman & 3 \\
\hline R234 & fluid jet & 1 \\
\hline R234 & carrier & 2 \\
\hline R234 & mechanic & 3 \\
\hline $\mathrm{R} 235$ & fireman & 1 \\
\hline $\mathrm{R} 235$ & brazier & 3 \\
\hline R236 & brazier & 1 \\
\hline R236 & painter & 2 \\
\hline R237 & crane operator & 1 \\
\hline R237 & mechanic & 2 \\
\hline $\mathrm{R} 237$ & safety & 3 \\
\hline R238 & general services & 1 \\
\hline R238 & painter & 2 \\
\hline $\mathrm{R} 238$ & welder & 3 \\
\hline R239 & general services & 1 \\
\hline R239 & welder & 2 \\
\hline R239 & brazier & 3 \\
\hline R240 & machine operator & 1 \\
\hline $\mathrm{R} 240$ & carpenter & 2 \\
\hline R241 & brazier & 1 \\
\hline R241 & general services & 2 \\
\hline $\mathrm{R} 241$ & painter & 3 \\
\hline R242 & carrier & 1 \\
\hline R242 & carpenter & 2 \\
\hline R242 & assembler & 3 \\
\hline R243 & carpenter & 1 \\
\hline R243 & crane operator & 2 \\
\hline R243 & assembler & 3 \\
\hline R244 & plumber & 1 \\
\hline R244 & blow torched & 2 \\
\hline R244 & carrier & 3 \\
\hline R245 & machine operator & 1 \\
\hline
\end{tabular}




\begin{tabular}{|c|c|c|}
\hline $\mathrm{R} 245$ & support & 2 \\
\hline R245 & fluid jet & 3 \\
\hline R246 & assembler scaffolding & 1 \\
\hline $\mathrm{R} 246$ & quality control & 2 \\
\hline R246 & carpenter & 3 \\
\hline R247 & dock aid & 1 \\
\hline R247 & brazier & 2 \\
\hline R247 & fluid jet & 3 \\
\hline R248 & fireman & 1 \\
\hline $\mathrm{R} 248$ & support & 2 \\
\hline R248 & assembler scaffolding & 3 \\
\hline R249 & brazier & 1 \\
\hline R249 & support & 2 \\
\hline R249 & machine operator & 3 \\
\hline $\mathrm{R} 250$ & quality control & 1 \\
\hline $\mathrm{R} 250$ & electrician & 2 \\
\hline $\mathrm{R} 250$ & blow torched & 3 \\
\hline $\mathrm{R} 251$ & support & 1 \\
\hline $\mathrm{R} 251$ & fireman & 2 \\
\hline $\mathrm{R} 251$ & quality control & 3 \\
\hline $\mathrm{R} 252$ & dock aid & 1 \\
\hline $\mathrm{R} 252$ & crane operator & 2 \\
\hline $\mathrm{R} 252$ & plumber & 3 \\
\hline R253 & fireman & 1 \\
\hline $\mathrm{R} 253$ & blow torched & 2 \\
\hline $\mathrm{R} 254$ & blow torched & 1 \\
\hline $\mathrm{R} 254$ & brazier & 2 \\
\hline $\mathrm{R} 254$ & painter & 3 \\
\hline $\mathrm{R} 255$ & general services & 1 \\
\hline $\mathrm{R} 255$ & quality control & 2 \\
\hline $\mathrm{R} 256$ & quality control & 1 \\
\hline $\mathrm{R} 256$ & safety & 2 \\
\hline $\mathrm{R} 256$ & assembler scaffolding & 3 \\
\hline R257 & mechanic & 1 \\
\hline $\mathrm{R} 257$ & assembler & 3 \\
\hline $\mathrm{R} 258$ & mechanic & 1 \\
\hline $\mathrm{R} 258$ & welder & 2 \\
\hline R259 & carpenter & 1 \\
\hline $\mathrm{R} 259$ & carrier & 2 \\
\hline R260 & machine operator & 1 \\
\hline R260 & safety & 3 \\
\hline R261 & dock aid & 1 \\
\hline R261 & fireman & 2 \\
\hline R261 & mechanic & 3 \\
\hline
\end{tabular}




\begin{tabular}{|c|c|c|}
\hline R262 & brazier & 1 \\
\hline R262 & quality control & 2 \\
\hline R262 & electrician & 3 \\
\hline R263 & electrician & 1 \\
\hline R263 & brazier & 2 \\
\hline R263 & carrier & 3 \\
\hline R264 & plumber & 1 \\
\hline R264 & assembler scaffolding & 2 \\
\hline R264 & assembler & 3 \\
\hline R265 & dock aid & 1 \\
\hline R265 & carrier & 2 \\
\hline R265 & support & 3 \\
\hline R266 & blow torched & 1 \\
\hline R266 & carpenter & 2 \\
\hline R266 & assembler & 3 \\
\hline R267 & dock aid & 1 \\
\hline R267 & machine operator & 2 \\
\hline R267 & plumber & 3 \\
\hline R268 & welder & 1 \\
\hline R268 & assembler scaffolding & 2 \\
\hline R268 & safety & 3 \\
\hline R269 & mechanic & 1 \\
\hline R269 & blow torched & 2 \\
\hline R269 & carrier & 3 \\
\hline $\mathrm{R} 270$ & dock aid & 1 \\
\hline $\mathrm{R} 270$ & carpenter & 2 \\
\hline $\mathrm{R} 270$ & carrier & 3 \\
\hline $\mathrm{R} 271$ & support & 1 \\
\hline $\mathrm{R} 271$ & machine operator & 2 \\
\hline $\mathrm{R} 271$ & carrier & 3 \\
\hline $\mathrm{R} 272$ & assembler & 1 \\
\hline R272 & painter & 2 \\
\hline R272 & electrician & 3 \\
\hline $\mathrm{R} 273$ & blow torched & 1 \\
\hline $\mathrm{R} 273$ & general services & 2 \\
\hline R273 & safety & 3 \\
\hline R274 & carpenter & 1 \\
\hline R274 & blow torched & 2 \\
\hline $\mathrm{R} 274$ & machine operator & 3 \\
\hline $\mathrm{R} 275$ & assembler & 1 \\
\hline $\mathrm{R} 275$ & machine operator & 2 \\
\hline $\mathrm{R} 275$ & dock aid & 3 \\
\hline $\mathrm{R} 276$ & brazier & 1 \\
\hline $\mathrm{R} 276$ & general services & 2 \\
\hline
\end{tabular}




\begin{tabular}{|c|c|c|}
\hline R276 & crane operator & 3 \\
\hline R277 & fireman & 1 \\
\hline R277 & brazier & 2 \\
\hline R277 & dock aid & 3 \\
\hline R278 & crane operator & 1 \\
\hline $\mathrm{R} 278$ & painter & 2 \\
\hline R278 & brazier & 3 \\
\hline R279 & fireman & 1 \\
\hline R279 & electrician & 2 \\
\hline R279 & crane operator & 3 \\
\hline $\mathrm{R} 280$ & carrier & 1 \\
\hline $\mathrm{R} 280$ & crane operator & 2 \\
\hline $\mathrm{R} 280$ & electrician & 3 \\
\hline R281 & brazier & 1 \\
\hline R281 & support & 2 \\
\hline R281 & crane operator & 3 \\
\hline $\mathrm{R} 282$ & assembler scaffolding & 1 \\
\hline R282 & mechanic & 2 \\
\hline R282 & carrier & 3 \\
\hline $\mathrm{R} 283$ & carrier & 1 \\
\hline R283 & assembler scaffolding & 2 \\
\hline $\mathrm{R} 283$ & support & 3 \\
\hline R284 & safety & 1 \\
\hline R284 & painter & 2 \\
\hline $\mathrm{R} 284$ & carpenter & 3 \\
\hline $\mathrm{R} 285$ & machine operator & 1 \\
\hline $\mathrm{R} 285$ & general services & 2 \\
\hline $\mathrm{R} 285$ & painter & 3 \\
\hline R286 & dock aid & 1 \\
\hline R286 & carpenter & 2 \\
\hline R286 & welder & 3 \\
\hline $\mathrm{R} 287$ & brazier & 1 \\
\hline R287 & carpenter & 2 \\
\hline R287 & assembler & 3 \\
\hline R288 & painter & 1 \\
\hline R288 & assembler scaffolding & 2 \\
\hline R288 & dock aid & 3 \\
\hline R289 & carrier & 1 \\
\hline R289 & dock aid & 2 \\
\hline R289 & plumber & 3 \\
\hline $\mathrm{R} 290$ & general services & 1 \\
\hline R290 & dock aid & 2 \\
\hline R290 & assembler & 3 \\
\hline R291 & support & 1 \\
\hline
\end{tabular}




\begin{tabular}{|c|c|c|}
\hline R291 & welder & 2 \\
\hline R291 & assembler scaffolding & 3 \\
\hline R292 & crane operator & 1 \\
\hline R292 & mechanic & 2 \\
\hline R292 & blow torched & 3 \\
\hline R293 & electrician & 1 \\
\hline R293 & fireman & 2 \\
\hline R293 & carrier & 3 \\
\hline R294 & carrier & 1 \\
\hline R294 & fireman & 2 \\
\hline $\mathrm{R} 295$ & quality control & 1 \\
\hline R295 & machine operator & 2 \\
\hline R296 & assembler scaffolding & 1 \\
\hline R296 & crane operator & 2 \\
\hline R296 & dock aid & 3 \\
\hline R297 & assembler scaffolding & 1 \\
\hline R297 & dock aid & 2 \\
\hline R297 & plumber & 3 \\
\hline R298 & fluid jet & 1 \\
\hline R298 & general services & 2 \\
\hline R298 & dock aid & 3 \\
\hline R299 & support & 1 \\
\hline R299 & carrier & 2 \\
\hline R299 & assembler scaffolding & 3 \\
\hline R300 & carrier & 1 \\
\hline R300 & brazier & 2 \\
\hline R300 & mechanic & 3 \\
\hline R301 & fluid jet & 1 \\
\hline R301 & machine operator & 2 \\
\hline R301 & quality control & 3 \\
\hline R302 & blow torched & 1 \\
\hline R302 & plumber & 2 \\
\hline R302 & general services & 3 \\
\hline R303 & fireman & 1 \\
\hline R303 & electrician & 3 \\
\hline R304 & dock aid & 1 \\
\hline R304 & support & 2 \\
\hline R304 & fluid jet & 3 \\
\hline R305 & machine operator & 1 \\
\hline R305 & general services & 2 \\
\hline R305 & painter & 3 \\
\hline R306 & machine operator & 1 \\
\hline R306 & brazier & 2 \\
\hline R306 & mechanic & 3 \\
\hline
\end{tabular}




\begin{tabular}{|c|c|c|}
\hline R307 & electrician & 1 \\
\hline R307 & carrier & 2 \\
\hline R307 & fluid jet & 3 \\
\hline R308 & quality control & 1 \\
\hline R308 & carrier & 2 \\
\hline R308 & machine operator & 3 \\
\hline R309 & general services & 1 \\
\hline R309 & blow torched & 2 \\
\hline R310 & fireman & 1 \\
\hline R310 & blow torched & 2 \\
\hline R310 & carrier & 3 \\
\hline R311 & machine operator & 1 \\
\hline R311 & plumber & 2 \\
\hline R312 & electrician & 1 \\
\hline R312 & machine operator & 2 \\
\hline R313 & electrician & 1 \\
\hline R313 & blow torched & 2 \\
\hline R313 & plumber & 3 \\
\hline R314 & blow torched & 1 \\
\hline R314 & support & 2 \\
\hline R315 & safety & 1 \\
\hline R315 & quality control & 2 \\
\hline R316 & carrier & 1 \\
\hline R316 & brazier & 2 \\
\hline R317 & crane operator & 1 \\
\hline R317 & safety & 2 \\
\hline R317 & electrician & 3 \\
\hline R318 & electrician & 1 \\
\hline R318 & brazier & 2 \\
\hline R318 & welder & 3 \\
\hline R319 & carpenter & 1 \\
\hline R319 & painter & 2 \\
\hline R319 & general services & 3 \\
\hline R320 & fireman & 1 \\
\hline R320 & machine operator & 2 \\
\hline R320 & welder & 3 \\
\hline R321 & safety & 1 \\
\hline R321 & fireman & 2 \\
\hline R321 & machine operator & 3 \\
\hline R322 & carrier & 1 \\
\hline R322 & carpenter & 2 \\
\hline R322 & general services & 3 \\
\hline R323 & assembler scaffolding & 1 \\
\hline R323 & carpenter & 2 \\
\hline
\end{tabular}




\begin{tabular}{|c|c|c|}
\hline R323 & crane operator & 3 \\
\hline R324 & general services & 1 \\
\hline R324 & quality control & 2 \\
\hline R325 & assembler scaffolding & 1 \\
\hline R325 & quality control & 2 \\
\hline R325 & electrician & 3 \\
\hline R326 & assembler scaffolding & 1 \\
\hline R326 & safety & 2 \\
\hline R326 & general services & 3 \\
\hline R327 & machine operator & 1 \\
\hline R327 & fluid jet & 3 \\
\hline R328 & fluid jet & 1 \\
\hline R328 & assembler & 2 \\
\hline R328 & dock aid & 3 \\
\hline R329 & quality control & 1 \\
\hline R329 & assembler & 2 \\
\hline R329 & machine operator & 3 \\
\hline R330 & safety & 1 \\
\hline R330 & carrier & 2 \\
\hline R330 & plumber & 3 \\
\hline R331 & carpenter & 1 \\
\hline R331 & carrier & 2 \\
\hline R331 & painter & 3 \\
\hline R332 & brazier & 1 \\
\hline R332 & quality control & 2 \\
\hline R332 & assembler scaffolding & 3 \\
\hline R333 & safety & 1 \\
\hline R333 & blow torched & 2 \\
\hline R333 & quality control & 3 \\
\hline R334 & painter & 1 \\
\hline R334 & support & 2 \\
\hline R334 & assembler & 3 \\
\hline R335 & assembler & 1 \\
\hline R335 & support & 2 \\
\hline R335 & fireman & 3 \\
\hline R336 & crane operator & 1 \\
\hline R336 & electrician & 2 \\
\hline R336 & assembler scaffolding & 3 \\
\hline R337 & safety & 1 \\
\hline R337 & blow torched & 2 \\
\hline R337 & electrician & 3 \\
\hline R338 & fluid jet & 1 \\
\hline R338 & electrician & 2 \\
\hline R338 & carrier & 3 \\
\hline
\end{tabular}




\begin{tabular}{|c|c|c|}
\hline R339 & crane operator & 1 \\
\hline R339 & blow torched & 2 \\
\hline R339 & dock aid & 3 \\
\hline R340 & fireman & 1 \\
\hline R340 & assembler & 2 \\
\hline R340 & fluid jet & 3 \\
\hline R341 & blow torched & 1 \\
\hline R341 & brazier & 2 \\
\hline R341 & machine operator & 3 \\
\hline R342 & painter & 1 \\
\hline R342 & fireman & 2 \\
\hline R342 & crane operator & 3 \\
\hline R343 & machine operator & 1 \\
\hline R343 & blow torched & 2 \\
\hline R343 & safety & 3 \\
\hline R344 & plumber & 1 \\
\hline R344 & fluid jet & 3 \\
\hline R345 & painter & 1 \\
\hline R345 & blow torched & 2 \\
\hline R345 & general services & 3 \\
\hline R346 & assembler & 1 \\
\hline R346 & fireman & 2 \\
\hline R346 & support & 3 \\
\hline R347 & quality control & 1 \\
\hline R347 & dock aid & 2 \\
\hline R348 & carrier & 1 \\
\hline R348 & crane operator & 2 \\
\hline R348 & dock aid & 3 \\
\hline R349 & welder & 1 \\
\hline R349 & mechanic & 2 \\
\hline R350 & fluid jet & 1 \\
\hline R350 & plumber & 2 \\
\hline R350 & blow torched & 3 \\
\hline R351 & assembler scaffolding & 1 \\
\hline R351 & dock aid & 2 \\
\hline R351 & carrier & 3 \\
\hline R352 & blow torched & 1 \\
\hline R352 & fireman & 2 \\
\hline R352 & plumber & 3 \\
\hline R353 & fluid jet & 1 \\
\hline R353 & carrier & 2 \\
\hline R353 & assembler scaffolding & 3 \\
\hline R354 & support & 1 \\
\hline R354 & quality control & 3 \\
\hline
\end{tabular}




\begin{tabular}{|c|c|c|}
\hline R355 & safety & 1 \\
\hline R355 & assembler & 2 \\
\hline R356 & mechanic & 1 \\
\hline R356 & welder & 2 \\
\hline R356 & dock aid & 3 \\
\hline R357 & blow torched & 1 \\
\hline R357 & fireman & 2 \\
\hline R358 & assembler scaffolding & 1 \\
\hline R358 & brazier & 2 \\
\hline R358 & mechanic & 3 \\
\hline R359 & assembler scaffolding & 1 \\
\hline R359 & general services & 2 \\
\hline R359 & plumber & 3 \\
\hline R360 & carpenter & 1 \\
\hline R360 & electrician & 2 \\
\hline R361 & machine operator & 1 \\
\hline R361 & assembler scaffolding & 2 \\
\hline R361 & welder & 3 \\
\hline R362 & carpenter & 1 \\
\hline R362 & assembler scaffolding & 2 \\
\hline R362 & safety & 3 \\
\hline R363 & machine operator & 1 \\
\hline R363 & assembler scaffolding & 2 \\
\hline R363 & mechanic & 3 \\
\hline R364 & safety & 1 \\
\hline R364 & dock aid & 2 \\
\hline R364 & welder & 3 \\
\hline R365 & machine operator & 1 \\
\hline R365 & fluid jet & 2 \\
\hline R365 & assembler scaffolding & 3 \\
\hline R366 & assembler scaffolding & 1 \\
\hline R366 & general services & 2 \\
\hline R367 & painter & 1 \\
\hline R367 & plumber & 2 \\
\hline R367 & dock aid & 3 \\
\hline R368 & fluid jet & 1 \\
\hline R368 & dock aid & 2 \\
\hline R368 & fireman & 3 \\
\hline R369 & safety & 1 \\
\hline R369 & blow torched & 2 \\
\hline R369 & quality control & 3 \\
\hline R370 & safety & 1 \\
\hline R370 & fluid jet & 2 \\
\hline R370 & machine operator & 3 \\
\hline
\end{tabular}




\begin{tabular}{|c|c|c|}
\hline R371 & blow torched & 1 \\
\hline R371 & assembler & 2 \\
\hline R371 & support & 3 \\
\hline R372 & fluid jet & 1 \\
\hline R372 & general services & 2 \\
\hline R373 & safety & 1 \\
\hline R373 & crane operator & 2 \\
\hline R373 & machine operator & 3 \\
\hline R374 & carrier & 1 \\
\hline R374 & plumber & 2 \\
\hline R374 & brazier & 3 \\
\hline R375 & support & 1 \\
\hline R375 & machine operator & 2 \\
\hline R375 & plumber & 3 \\
\hline R376 & quality control & 1 \\
\hline R376 & mechanic & 2 \\
\hline R376 & blow torched & 3 \\
\hline R377 & mechanic & 1 \\
\hline R377 & brazier & 2 \\
\hline R377 & assembler & 3 \\
\hline R378 & plumber & 1 \\
\hline R378 & dock aid & 2 \\
\hline R378 & assembler scaffolding & 3 \\
\hline R379 & quality control & 1 \\
\hline R379 & safety & 2 \\
\hline R379 & plumber & 3 \\
\hline R380 & general services & 1 \\
\hline R380 & painter & 2 \\
\hline R380 & fluid jet & 3 \\
\hline R381 & welder & 1 \\
\hline R381 & painter & 2 \\
\hline R381 & fluid jet & 3 \\
\hline R382 & assembler & 1 \\
\hline R382 & general services & 2 \\
\hline R382 & machine operator & 3 \\
\hline R383 & assembler & 1 \\
\hline R383 & assembler scaffolding & 2 \\
\hline R383 & brazier & 3 \\
\hline R384 & fireman & 1 \\
\hline R384 & general services & 2 \\
\hline R384 & electrician & 3 \\
\hline R385 & support & 1 \\
\hline R385 & blow torched & 2 \\
\hline R385 & assembler & 3 \\
\hline
\end{tabular}




\begin{tabular}{|c|c|c|}
\hline R386 & support & 1 \\
\hline R386 & carpenter & 2 \\
\hline R386 & mechanic & 3 \\
\hline R387 & fireman & 1 \\
\hline R387 & blow torched & 2 \\
\hline R388 & welder & 1 \\
\hline R388 & blow torched & 2 \\
\hline R388 & mechanic & 3 \\
\hline R389 & brazier & 1 \\
\hline R389 & dock aid & 2 \\
\hline R389 & machine operator & 3 \\
\hline R390 & painter & 1 \\
\hline R390 & safety & 2 \\
\hline R391 & mechanic & 1 \\
\hline R391 & electrician & 2 \\
\hline R392 & brazier & 1 \\
\hline R392 & support & 2 \\
\hline R392 & machine operator & 3 \\
\hline R393 & general services & 1 \\
\hline R393 & dock aid & 2 \\
\hline R393 & plumber & 3 \\
\hline R394 & general services & 1 \\
\hline R394 & machine operator & 2 \\
\hline R394 & assembler & 3 \\
\hline R395 & plumber & 1 \\
\hline R395 & fluid jet & 2 \\
\hline R395 & blow torched & 3 \\
\hline R396 & electrician & 1 \\
\hline R396 & carpenter & 2 \\
\hline R397 & mechanic & 1 \\
\hline R397 & support & 2 \\
\hline R397 & crane operator & 3 \\
\hline R398 & safety & 1 \\
\hline R398 & fluid jet & 2 \\
\hline R398 & carrier & 3 \\
\hline R399 & support & 1 \\
\hline R399 & dock aid & 2 \\
\hline R399 & carrier & 3 \\
\hline $\mathrm{R} 400$ & general services & 1 \\
\hline $\mathrm{R} 400$ & quality control & 2 \\
\hline $\mathrm{R} 400$ & fluid jet & 3 \\
\hline R401 & assembler scaffolding & 1 \\
\hline R401 & fluid jet & 2 \\
\hline $\mathrm{R} 402$ & support & 1 \\
\hline
\end{tabular}




\begin{tabular}{|c|c|c|}
\hline R402 & crane operator & 2 \\
\hline R402 & fireman & 3 \\
\hline R403 & welder & 1 \\
\hline R403 & brazier & 2 \\
\hline R403 & quality control & 3 \\
\hline R404 & assembler scaffolding & 1 \\
\hline R404 & general services & 2 \\
\hline R404 & safety & 3 \\
\hline $\mathrm{R} 405$ & crane operator & 1 \\
\hline R405 & dock aid & 2 \\
\hline R406 & fluid jet & 1 \\
\hline R406 & brazier & 2 \\
\hline R406 & assembler scaffolding & 3 \\
\hline R407 & painter & 1 \\
\hline R407 & fireman & 2 \\
\hline R407 & support & 3 \\
\hline R408 & crane operator & 1 \\
\hline R408 & assembler & 2 \\
\hline R408 & support & 3 \\
\hline R409 & safety & 1 \\
\hline R409 & dock aid & 2 \\
\hline $\mathrm{R} 410$ & crane operator & 1 \\
\hline $\mathrm{R} 410$ & blow torched & 2 \\
\hline R410 & plumber & 3 \\
\hline R411 & assembler & 1 \\
\hline R411 & support & 2 \\
\hline R411 & quality control & 3 \\
\hline R412 & assembler scaffolding & 1 \\
\hline R412 & painter & 2 \\
\hline R412 & crane operator & 3 \\
\hline R413 & machine operator & 1 \\
\hline R413 & crane operator & 2 \\
\hline R413 & plumber & 3 \\
\hline R414 & electrician & 1 \\
\hline R414 & welder & 2 \\
\hline R414 & fireman & 3 \\
\hline $\mathrm{R} 415$ & fluid jet & 1 \\
\hline $\mathrm{R} 415$ & support & 2 \\
\hline $\mathrm{R} 415$ & quality control & 3 \\
\hline R416 & welder & 1 \\
\hline R416 & assembler scaffolding & 2 \\
\hline R416 & plumber & 3 \\
\hline R417 & blow torched & 1 \\
\hline $\mathrm{R} 417$ & fluid jet & 2 \\
\hline
\end{tabular}




\begin{tabular}{|c|c|c|}
\hline $\mathrm{R} 417$ & machine operator & 3 \\
\hline R418 & fluid jet & 1 \\
\hline R418 & assembler scaffolding & 2 \\
\hline R418 & blow torched & 3 \\
\hline R419 & painter & 1 \\
\hline R419 & crane operator & 2 \\
\hline R419 & safety & 3 \\
\hline $\mathrm{R} 420$ & safety & 1 \\
\hline $\mathrm{R} 420$ & crane operator & 2 \\
\hline $\mathrm{R} 420$ & carrier & 3 \\
\hline $\mathrm{R} 421$ & quality control & 1 \\
\hline R421 & blow torched & 2 \\
\hline R421 & assembler & 3 \\
\hline $\mathrm{R} 422$ & painter & 1 \\
\hline $\mathrm{R} 422$ & electrician & 2 \\
\hline R422 & brazier & 3 \\
\hline R423 & mechanic & 1 \\
\hline R423 & assembler scaffolding & 2 \\
\hline $\mathrm{R} 423$ & blow torched & 3 \\
\hline $\mathrm{R} 424$ & painter & 1 \\
\hline R424 & brazier & 2 \\
\hline R424 & mechanic & 3 \\
\hline $\mathrm{R} 425$ & dock aid & 1 \\
\hline $\mathrm{R} 425$ & carpenter & 2 \\
\hline $\mathrm{R} 425$ & brazier & 3 \\
\hline $\mathrm{R} 426$ & machine operator & 1 \\
\hline $\mathrm{R} 426$ & blow torched & 2 \\
\hline $\mathrm{R} 427$ & machine operator & 1 \\
\hline R427 & blow torched & 2 \\
\hline R427 & assembler & 3 \\
\hline R428 & dock aid & 1 \\
\hline R428 & blow torched & 2 \\
\hline $\mathrm{R} 428$ & support & 3 \\
\hline R429 & brazier & 1 \\
\hline R429 & safety & 2 \\
\hline R429 & assembler & 3 \\
\hline $\mathrm{R} 430$ & blow torched & 1 \\
\hline $\mathrm{R} 430$ & painter & 2 \\
\hline R431 & plumber & 1 \\
\hline R431 & blow torched & 2 \\
\hline R431 & general services & 3 \\
\hline R432 & support & 1 \\
\hline $\mathrm{R} 432$ & plumber & 2 \\
\hline $\mathrm{R} 432$ & blow torched & 3 \\
\hline
\end{tabular}




\begin{tabular}{|c|c|c|}
\hline $\mathrm{R} 433$ & mechanic & 1 \\
\hline R433 & quality control & 2 \\
\hline R433 & painter & 3 \\
\hline R434 & fireman & 1 \\
\hline R434 & crane operator & 2 \\
\hline R434 & assembler scaffolding & 3 \\
\hline R435 & general services & 1 \\
\hline $\mathrm{R} 435$ & brazier & 2 \\
\hline $\mathrm{R} 435$ & safety & 3 \\
\hline R436 & plumber & 1 \\
\hline $\mathrm{R} 436$ & safety & 2 \\
\hline R436 & brazier & 3 \\
\hline $\mathrm{R} 437$ & plumber & 1 \\
\hline R437 & machine operator & 2 \\
\hline R437 & dock aid & 3 \\
\hline R438 & electrician & 1 \\
\hline R438 & mechanic & 2 \\
\hline R438 & assembler scaffolding & 3 \\
\hline R439 & quality control & 1 \\
\hline R439 & machine operator & 2 \\
\hline R439 & carpenter & 3 \\
\hline $\mathrm{R} 440$ & fluid jet & 1 \\
\hline $\mathrm{R} 440$ & blow torched & 2 \\
\hline $\mathrm{R} 440$ & general services & 3 \\
\hline R441 & general services & 1 \\
\hline R441 & carrier & 2 \\
\hline R441 & machine operator & 3 \\
\hline R442 & painter & 1 \\
\hline R442 & brazier & 2 \\
\hline R442 & electrician & 3 \\
\hline R443 & brazier & 1 \\
\hline R443 & fireman & 2 \\
\hline R443 & machine operator & 3 \\
\hline R444 & carpenter & 1 \\
\hline R444 & blow torched & 2 \\
\hline R444 & dock aid & 3 \\
\hline $\mathrm{R} 445$ & general services & 1 \\
\hline $\mathrm{R} 445$ & blow torched & 2 \\
\hline R446 & blow torched & 1 \\
\hline R446 & quality control & 2 \\
\hline R446 & safety & 3 \\
\hline R447 & crane operator & 1 \\
\hline R447 & assembler & 2 \\
\hline R447 & welder & 3 \\
\hline
\end{tabular}




\begin{tabular}{|c|c|c|}
\hline $\mathrm{R} 448$ & support & 1 \\
\hline R448 & blow torched & 2 \\
\hline R449 & assembler & 1 \\
\hline R449 & dock aid & 2 \\
\hline R449 & blow torched & 3 \\
\hline $\mathrm{R} 450$ & mechanic & 1 \\
\hline $\mathrm{R} 450$ & welder & 2 \\
\hline $\mathrm{R} 450$ & dock aid & 3 \\
\hline $\mathrm{R} 451$ & general services & 1 \\
\hline R451 & assembler & 2 \\
\hline $\mathrm{R} 452$ & welder & 1 \\
\hline $\mathrm{R} 452$ & support & 2 \\
\hline R452 & fireman & 3 \\
\hline R453 & welder & 1 \\
\hline R453 & blow torched & 2 \\
\hline R453 & fluid jet & 3 \\
\hline $\mathrm{R} 454$ & assembler scaffolding & 1 \\
\hline R454 & brazier & 2 \\
\hline $\mathrm{R} 454$ & blow torched & 3 \\
\hline $\mathrm{R} 455$ & general services & 1 \\
\hline $\mathrm{R} 455$ & crane operator & 2 \\
\hline $\mathrm{R} 455$ & quality control & 3 \\
\hline R456 & support & 1 \\
\hline R456 & quality control & 2 \\
\hline $\mathrm{R} 456$ & fluid jet & 3 \\
\hline R457 & assembler scaffolding & 1 \\
\hline R457 & carrier & 2 \\
\hline $\mathrm{R} 458$ & safety & 1 \\
\hline R458 & painter & 2 \\
\hline R459 & carpenter & 1 \\
\hline R459 & mechanic & 2 \\
\hline R459 & blow torched & 3 \\
\hline $\mathrm{R} 460$ & blow torched & 1 \\
\hline R460 & crane operator & 2 \\
\hline R460 & assembler scaffolding & 3 \\
\hline R461 & crane operator & 1 \\
\hline R461 & welder & 2 \\
\hline R461 & mechanic & 3 \\
\hline R462 & assembler & 1 \\
\hline R462 & welder & 2 \\
\hline R462 & fluid jet & 3 \\
\hline R463 & support & 1 \\
\hline R463 & fluid jet & 2 \\
\hline $\mathrm{R} 463$ & plumber & 3 \\
\hline
\end{tabular}




\begin{tabular}{|c|c|c|}
\hline R464 & quality control & 1 \\
\hline R464 & welder & 2 \\
\hline R464 & general services & 3 \\
\hline R465 & plumber & 1 \\
\hline R465 & welder & 2 \\
\hline R465 & crane operator & 3 \\
\hline R466 & carrier & 1 \\
\hline R466 & carpenter & 2 \\
\hline R466 & general services & 3 \\
\hline R467 & carpenter & 1 \\
\hline R467 & assembler scaffolding & 2 \\
\hline R467 & painter & 3 \\
\hline R468 & plumber & 1 \\
\hline R468 & welder & 3 \\
\hline R469 & blow torched & 1 \\
\hline R469 & dock aid & 2 \\
\hline R469 & safety & 3 \\
\hline R470 & dock aid & 1 \\
\hline R470 & general services & 2 \\
\hline R470 & blow torched & 3 \\
\hline R471 & carpenter & 1 \\
\hline R471 & mechanic & 2 \\
\hline R471 & blow torched & 3 \\
\hline R472 & painter & 1 \\
\hline R472 & crane operator & 2 \\
\hline R472 & carrier & 3 \\
\hline R473 & crane operator & 1 \\
\hline R473 & quality control & 2 \\
\hline R474 & electrician & 1 \\
\hline R474 & carpenter & 2 \\
\hline R474 & support & 3 \\
\hline R475 & fluid jet & 1 \\
\hline R475 & carrier & 2 \\
\hline R475 & blow torched & 3 \\
\hline
\end{tabular}




\begin{tabular}{|c|c|c|c|c|c|c|c|c|}
\hline \multicolumn{9}{|c|}{ Table AIV.4: Cost and Schedule } \\
\hline $\mathbf{T}$ & $\begin{array}{l}\text { Due } \\
\text { Date }\end{array}$ & $\begin{array}{l}\text { Start } \\
\text { Date }\end{array}$ & $\begin{array}{l}\text { End } \\
\text { Date }\end{array}$ & D. & $\begin{array}{c}\text { Fixed } \\
\text { Cost }\end{array}$ & $\begin{array}{c}\text { Variable } \\
\text { Cost }\end{array}$ & $\begin{array}{c}\text { Penalty } \\
\text { Cost }\end{array}$ & Bonus \\
\hline 1 & $6 / 3 / 2015$ & $5 / 18 / 2015$ & $5 / 20 / 2015$ & -13.29 & $\$ 446.0$ & $\$ 27,360.23$ & $\$ 0.00$ & $\$ 66,458.25$ \\
\hline 2 & $6 / 12 / 2015$ & $5 / 21 / 2015$ & $6 / 1 / 2015$ & -10.5 & $\$ 410.00$ & $\$ 128,640.27$ & $\$ 0.00$ & $\$ 52,499.85$ \\
\hline 3 & $6 / 27 / 2015$ & $6 / 1 / 2015$ & $6 / 15 / 2015$ & -11.29 & $\$ 111.00$ & $\$ 40,800.10$ & $\$ 0.00$ & $\$ 56,458.10$ \\
\hline 4 & $7 / 10 / 2015$ & $6 / 16 / 2015$ & $6 / 26 / 2015$ & -13.29 & $\$ 658.00$ & $\$ 179,280.69$ & $\$ 0.00$ & $\$ 66,458.15$ \\
\hline 5 & $7 / 10 / 2015$ & $6 / 29 / 2015$ & $6 / 29 / 2015$ & -10.63 & $\$ 54.00$ & $\$ 60.00$ & $\$ 0.00$ & $\$ 53,125.00$ \\
\hline 6 & $7 / 11 / 2015$ & $6 / 29 / 2015$ & $6 / 29 / 2015$ & -11.54 & $\$ 55.00$ & $\$ 120.01$ & $\$ 0.00$ & $\$ 57,708.30$ \\
\hline 7 & $5 / 31 / 2015$ & $5 / 18 / 2015$ & $5 / 19 / 2015$ & -11.5 & $\$ 117.00$ & $\$ 3,360.04$ & $\$ 0.00$ & $\$ 57,499.95$ \\
\hline 8 & $6 / 11 / 2015$ & $5 / 19 / 2015$ & $5 / 28 / 2015$ & -13.29 & $\$ 472.00$ & $\$ 106,080.31$ & $\$ 0.00$ & $\$ 66,458.15$ \\
\hline 9 & $6 / 25 / 2015$ & $5 / 29 / 2015$ & $6 / 12 / 2015$ & -12.5 & $\$ 111.00$ & $\$ 40,800.12$ & $\$ 0.00$ & $\$ 62,499.80$ \\
\hline 10 & $7 / 5 / 2015$ & $6 / 12 / 2015$ & $6 / 23 / 2015$ & -11.29 & $\$ 642.00$ & $\$ 192,960.40$ & $\$ 0.00$ & $\$ 56,458.15$ \\
\hline 11 & $7 / 7 / 2015$ & $6 / 24 / 2015$ & $6 / 24 / 2015$ & -12.63 & $\$ 52.00$ & $\$ 60.00$ & $\$ 0.00$ & $\$ 63,125.00$ \\
\hline 12 & $5 / 29 / 2015$ & $5 / 18 / 2015$ & $5 / 18 / 2015$ & -10.63 & $\$ 59.00$ & $\$ 60.00$ & $\$ 0.00$ & $\$ 53,125.00$ \\
\hline 13 & $6 / 10 / 2015$ & $5 / 18 / 2015$ & $5 / 27 / 2015$ & -13.42 & $\$ 448.00$ & $\$ 106,080.31$ & $\$ 0.00$ & $\$ 67,083.20$ \\
\hline 14 & $7 / 9 / 2015$ & $5 / 27 / 2015$ & $6 / 25 / 2015$ & -13.42 & $\$ 104.00$ & $\$ 83,520.20$ & $\$ 0.00$ & $\$ 67,082.85$ \\
\hline 15 & $7 / 21 / 2015$ & $6 / 25 / 2015$ & $7 / 7 / 2015$ & -13.62 & $\$ 616.00$ & $\$ 203,760.40$ & $\$ 0.00$ & $\$ 68,124.40$ \\
\hline 16 & $7 / 22 / 2015$ & $7 / 7 / 2015$ & $7 / 7 / 2015$ & -14.54 & $\$ 103.00$ & $\$ 240.02$ & $\$ 0.00$ & $\$ 72,707.70$ \\
\hline 17 & $6 / 4 / 2015$ & $5 / 18 / 2015$ & $5 / 22 / 2015$ & -12.5 & $\$ 54.00$ & $\$ 6,000.03$ & $\$ 0.00$ & $\$ 62,499.90$ \\
\hline 18 & $7 / 1 / 2015$ & $5 / 22 / 2015$ & $6 / 18 / 2015$ & -12.58 & $\$ 462.00$ & $\$ 309,600.69$ & $\$ 0.00$ & $\$ 62,916.30$ \\
\hline 19 & $7 / 16 / 2015$ & $6 / 18 / 2015$ & $7 / 17 / 2015$ & 1.42 & $\$ 60.00$ & $\$ 41,760.10$ & $\$ 14,168.10$ & $\$ 0.00$ \\
\hline 20 & $7 / 24 / 2015$ & $7 / 17 / 2015$ & $7 / 27 / 2015$ & 3.42 & $\$ 736.00$ & $\$ 201,600.40$ & $\$ 34,168.30$ & $\$ 0.00$ \\
\hline 21 & $7 / 17 / 2015$ & $6 / 19 / 2015$ & $7 / 6 / 2015$ & -10.29 & $\$ 1,112.00$ & $\$ 500,401.44$ & $\$ 0.00$ & $\$ 51,458.10$ \\
\hline 22 & $5 / 31 / 2015$ & $5 / 18 / 2015$ & $5 / 19 / 2015$ & -11.5 & $\$ 171.00$ & $\$ 5,040.06$ & $\$ 0.00$ & $\$ 57,499.95$ \\
\hline 23 & $5 / 31 / 2015$ & $5 / 18 / 2015$ & $5 / 20 / 2015$ & -10.29 & $\$ 105.00$ & $\$ 6,840.06$ & $\$ 0.00$ & $\$ 51,458.25$ \\
\hline 24 & $5 / 31 / 2015$ & $5 / 18 / 2015$ & $5 / 20 / 2015$ & -10.29 & $\$ 59.00$ & $\$ 3,420.03$ & $\$ 0.00$ & $\$ 51,458.25$ \\
\hline 25 & $6 / 9 / 2015$ & $5 / 18 / 2015$ & $6 / 1 / 2015$ & -7.5 & $\$ 548.00$ & $\$ 204,000.62$ & $\$ 0.00$ & $\$ 37,499.80$ \\
\hline 26 & $6 / 9 / 2015$ & $5 / 18 / 2015$ & $6 / 1 / 2015$ & -7.5 & $\$ 566.00$ & $\$ 204,000.62$ & $\$ 0.00$ & $\$ 37,499.80$ \\
\hline 27 & $6 / 21 / 2015$ & $6 / 1 / 2015$ & $6 / 10 / 2015$ & -10.29 & $\$ 2,232.00$ & $\$ 528,001.34$ & $\$ 0.00$ & $\$ 51,458.15$ \\
\hline 28 & $6 / 29 / 2015$ & $6 / 11 / 2015$ & $6 / 18 / 2015$ & -10.29 & $\$ 1,080.00$ & $\$ 212,400.86$ & $\$ 0.00$ & $\$ 51,458.20$ \\
\hline 29 & $7 / 7 / 2015$ & $6 / 11 / 2015$ & $6 / 26 / 2015$ & -10.29 & $\$ 571.00$ & $\$ 221,400.72$ & $\$ 0.00$ & $\$ 51,458.10$ \\
\hline 30 & $7 / 7 / 2015$ & $6 / 29 / 2015$ & $6 / 29 / 2015$ & -7.63 & $\$ 59.00$ & $\$ 60.00$ & $\$ 0.00$ & $\$ 38,125.00$ \\
\hline 31 & $7 / 12 / 2015$ & $6 / 29 / 2015$ & $7 / 1 / 2015$ & -10.29 & $\$ 110.00$ & $\$ 6,840.06$ & $\$ 0.00$ & $\$ 51,458.25$ \\
\hline 32 & $7 / 12 / 2015$ & $6 / 29 / 2015$ & $7 / 1 / 2015$ & -10.29 & $\$ 167.00$ & $\$ 10,260.09$ & $\$ 0.00$ & $\$ 51,458.25$ \\
\hline 33 & $5 / 28 / 2015$ & $5 / 18 / 2015$ & $5 / 19 / 2015$ & -8.5 & $\$ 172.00$ & $\$ 5,040.06$ & $\$ 0.00$ & $\$ 42,499.95$ \\
\hline 34 & $5 / 28 / 2015$ & $5 / 18 / 2015$ & $5 / 19 / 2015$ & -8.5 & $\$ 221.00$ & $\$ 6,720.08$ & $\$ 0.00$ & $\$ 42,499.95$ \\
\hline 35 & $6 / 11 / 2015$ & $6 / 1 / 2015$ & $6 / 2 / 2015$ & -8.29 & $\$ 1,109.00$ & $\$ 33,600.10$ & $\$ 0.00$ & $\$ 41,458.25$ \\
\hline 36 & $6 / 22 / 2015$ & $6 / 3 / 2015$ & $6 / 12 / 2015$ & -9.5 & $\$ 536.00$ & $\$ 132,000.34$ & $\$ 0.00$ & $\$ 47,499.85$ \\
\hline
\end{tabular}




\begin{tabular}{|c|c|c|c|c|c|c|c|c|}
\hline 37 & $7 / 2 / 2015$ & $6 / 12 / 2015$ & $6 / 23 / 2015$ & -8.29 & $\$ 534.00$ & $\$ 160,800.34$ & $\$ 0.00$ & $\$ 41,458.15$ \\
\hline 38 & 6/29/2015 & $6 / 12 / 2015$ & $6 / 18 / 2015$ & -10.29 & $\$ 1,080.00$ & $\$ 177,600.38$ & $\$ 0.00$ & $\$ 51,458.20$ \\
\hline 39 & $7 / 4 / 2015$ & $6 / 24 / 2015$ & $6 / 24 / 2015$ & -9.63 & $\$ 110.00$ & $\$ 120.01$ & $\$ 0.00$ & $\$ 48,125.00$ \\
\hline 40 & $7 / 5 / 2015$ & $6 / 24 / 2015$ & $6 / 24 / 2015$ & -10.54 & $\$ 167.00$ & $\$ 360.03$ & $\$ 0.00$ & $\$ 52,708.30$ \\
\hline 41 & $6 / 4 / 2015$ & $5 / 18 / 2015$ & $5 / 22 / 2015$ & -12.5 & $\$ 168.00$ & $\$ 18,000.10$ & $\$ 0.00$ & $\$ 62,499.90$ \\
\hline 42 & $6 / 14 / 2015$ & $5 / 22 / 2015$ & $6 / 2 / 2015$ & -11.29 & $\$ 110.00$ & $\$ 32,160.07$ & $\$ 0.00$ & $\$ 56,458.15$ \\
\hline 43 & $6 / 25 / 2015$ & $6 / 3 / 2015$ & $6 / 12 / 2015$ & -12.5 & $\$ 1,107.00$ & $\$ 264,000.67$ & $\$ 0.00$ & $\$ 62,499.85$ \\
\hline 44 & $7 / 5 / 2015$ & $6 / 12 / 2015$ & $6 / 23 / 2015$ & -11.29 & $\$ 553.00$ & $\$ 160,800.34$ & $\$ 0.00$ & $\$ 56,458.15$ \\
\hline 45 & $7 / 16 / 2015$ & $6 / 24 / 2015$ & 7/3/2015 & -12.5 & $\$ 533.00$ & $\$ 132,000.48$ & $\$ 0.00$ & $\$ 62,499.85$ \\
\hline 46 & $7 / 21 / 2015$ & 7/3/2015 & $7 / 8 / 2015$ & -12.5 & $\$ 172.00$ & $\$ 21,420.03$ & $\$ 0.00$ & $\$ 62,499.90$ \\
\hline 47 & $7 / 15 / 2015$ & $6 / 24 / 2015$ & $6 / 30 / 2015$ & -14.5 & $\$ 1,064.00$ & $\$ 177,600.67$ & $\$ 0.00$ & $\$ 72,499.90$ \\
\hline 48 & $5 / 31 / 2015$ & $5 / 18 / 2015$ & $5 / 19 / 2015$ & -11.5 & $\$ 515.00$ & $\$ 15,120.17$ & $\$ 0.00$ & $\$ 57,499.95$ \\
\hline 49 & $6 / 4 / 2015$ & $5 / 19 / 2015$ & $5 / 22 / 2015$ & -12.5 & $\$ 224.00$ & $\$ 17,280.06$ & $\$ 0.00$ & $\$ 62,499.90$ \\
\hline 50 & $7 / 2 / 2015$ & $6 / 4 / 2015$ & $6 / 12 / 2015$ & -19.42 & $\$ 1,114.00$ & $\$ 230,400.58$ & $\$ 0.00$ & $\$ 97,083.15$ \\
\hline 51 & $7 / 15 / 2015$ & $6 / 24 / 2015$ & 7/1/2015 & -13.29 & $\$ 539.00$ & $\$ 106,200.43$ & $\$ 0.00$ & $\$ 66,458.20$ \\
\hline 52 & $7 / 13 / 2015$ & $7 / 2 / 2015$ & $7 / 13 / 2015$ & 0.5 & $\$ 544.00$ & $\$ 160,800.48$ & $\$ 5,000.30$ & $\$ 0.00$ \\
\hline 53 & $7 / 15 / 2015$ & $7 / 13 / 2015$ & $7 / 14 / 2015$ & -0.29 & $\$ 342.00$ & $\$ 10,080.03$ & $\$ 0.00$ & $\$ 1,458.25$ \\
\hline 54 & $5 / 26 / 2015$ & $5 / 18 / 2015$ & $5 / 25 / 2015$ & -0.29 & $\$ 6,850.00$ & $\$ 1,327,505.40$ & $\$ 0.00$ & $\$ 1,458.20$ \\
\hline 55 & 6/13/2015 & $5 / 26 / 2015$ & 6/10/2015 & -2.29 & $\$ 774.00$ & $\$ 309,961.01$ & $\$ 0.00$ & $\$ 11,458.10$ \\
\hline 56 & $5 / 23 / 2015$ & $5 / 18 / 2015$ & $5 / 20 / 2015$ & -2.29 & $\$ 492.00$ & $\$ 30,780.26$ & $\$ 0.00$ & $\$ 11,458.25$ \\
\hline 57 & $5 / 23 / 2015$ & $5 / 21 / 2015$ & $5 / 21 / 2015$ & -1.58 & $\$ 54.00$ & $\$ 120.01$ & $\$ 0.00$ & $\$ 7,916.60$ \\
\hline 58 & $5 / 25 / 2015$ & $5 / 21 / 2015$ & $5 / 22 / 2015$ & -2.37 & $\$ 267.00$ & $\$ 8,700.05$ & $\$ 0.00$ & $\$ 11,874.90$ \\
\hline 59 & $5 / 31 / 2015$ & $5 / 22 / 2015$ & $5 / 29 / 2015$ & -1.58 & $\$ 2,180.00$ & $\$ 391,200.77$ & $\$ 0.00$ & $\$ 7,916.50$ \\
\hline 60 & $6 / 5 / 2015$ & $5 / 29 / 2015$ & $6 / 3 / 2015$ & -1.58 & $\$ 574.00$ & $\$ 72,000.14$ & $\$ 0.00$ & $\$ 7,916.45$ \\
\hline 61 & $6 / 26 / 2015$ & 6/30/2015 & 7/7/2015 & 11.71 & $\$ 1,107.00$ & $\$ 212,400.86$ & $\$ 117,083.60$ & $\$ 0.00$ \\
\hline 62 & 6/27/2015 & $7 / 8 / 2015$ & $7 / 8 / 2015$ & 11.38 & $\$ 60.00$ & $\$ 60.00$ & $\$ 113,750.00$ & $\$ 0.00$ \\
\hline 63 & 6/28/2015 & $7 / 8 / 2015$ & $7 / 8 / 2015$ & 10.46 & $\$ 60.00$ & $\$ 120.01$ & $\$ 104,583.40$ & $\$ 0.00$ \\
\hline 64 & $7 / 17 / 2015$ & $7 / 8 / 2015$ & $7 / 16 / 2015$ & -0.54 & $\$ 2,172.00$ & $\$ 460,801.15$ & $\$ 0.00$ & $\$ 2,708.20$ \\
\hline 65 & $7 / 18 / 2015$ & $7 / 16 / 2015$ & $7 / 17 / 2015$ & -0.33 & $\$ 342.00$ & $\$ 10,440.06$ & $\$ 0.00$ & $\$ 1,666.50$ \\
\hline 66 & $6 / 13 / 2015$ & $5 / 18 / 2015$ & $5 / 28 / 2015$ & -15.29 & $\$ 558.00$ & $\$ 149,400.58$ & $\$ 0.00$ & $\$ 76,458.15$ \\
\hline 67 & $6 / 22 / 2015$ & $5 / 29 / 2015$ & 6/9/2015 & -12.5 & $\$ 610.00$ & $\$ 176,880.53$ & $\$ 0.00$ & $\$ 62,499.85$ \\
\hline 68 & $7 / 2 / 2015$ & $6 / 8 / 2015$ & $6 / 18 / 2015$ & -13.29 & $\$ 54.00$ & $\$ 14,940.04$ & $\$ 0.00$ & $\$ 66,458.15$ \\
\hline 69 & $7 / 17 / 2015$ & $6 / 19 / 2015$ & $7 / 1 / 2015$ & -15.29 & $\$ 54.00$ & $\$ 17,820.06$ & $\$ 0.00$ & $\$ 76,458.15$ \\
\hline 70 & $7 / 20 / 2015$ & $7 / 2 / 2015$ & $7 / 6 / 2015$ & -13.29 & $\$ 54.00$ & $\$ 6,300.03$ & $\$ 0.00$ & $\$ 66,458.25$ \\
\hline 71 & $7 / 25 / 2015$ & 7/7/2015 & 7/9/2015 & -15.29 & $\$ 547.00$ & $\$ 34,200.14$ & $\$ 0.00$ & $\$ 76,458.25$ \\
\hline 72 & $7 / 25 / 2015$ & $7 / 10 / 2015$ & $7 / 22 / 2015$ & -2.29 & $\$ 550.00$ & $\$ 178,200.43$ & $\$ 0.00$ & $\$ 11,458.15$ \\
\hline 73 & $5 / 23 / 2015$ & $5 / 18 / 2015$ & $5 / 20 / 2015$ & -2.29 & $\$ 550.00$ & $\$ 34,200.29$ & $\$ 0.00$ & $\$ 11,458.25$ \\
\hline 74 & $5 / 24 / 2015$ & $5 / 21 / 2015$ & $5 / 22 / 2015$ & -1.5 & $\$ 610.00$ & $\$ 18,480.05$ & $\$ 0.00$ & $\$ 7,499.95$ \\
\hline 75 & $5 / 31 / 2015$ & $5 / 22 / 2015$ & $5 / 28 / 2015$ & -2.29 & $\$ 54.00$ & $\$ 8,940.02$ & $\$ 0.00$ & $\$ 11,458.20$ \\
\hline 76 & $6 / 6 / 2015$ & $5 / 29 / 2015$ & $6 / 4 / 2015$ & -1.5 & $\$ 54.00$ & $\$ 8,880.03$ & $\$ 0.00$ & $\$ 7,499.90$ \\
\hline 77 & $6 / 21 / 2015$ & $6 / 4 / 2015$ & $6 / 5 / 2015$ & -15.29 & $\$ 54.00$ & $\$ 1,680.00$ & $\$ 0.00$ & $\$ 76,458.25$ \\
\hline
\end{tabular}




\begin{tabular}{|c|c|c|c|c|c|c|c|c|}
\hline 78 & $6 / 21 / 2015$ & $6 / 8 / 2015$ & $6 / 8 / 2015$ & -12.58 & $\$ 541.00$ & $\$ 1,200.10$ & $\$ 0.00$ & $\$ 62,916.60$ \\
\hline 79 & $6 / 28 / 2015$ & $6 / 8 / 2015$ & $6 / 12 / 2015$ & -15.37 & $\$ 554.00$ & $\$ 60,600.24$ & $\$ 0.00$ & $\$ 76,874.85$ \\
\hline 80 & $5 / 18 / 2015$ & $5 / 18 / 2015$ & $5 / 18 / 2015$ & 0.38 & $\$ 888.00$ & $\$ 960.08$ & $\$ 3,750.00$ & $\$ 0.00$ \\
\hline 81 & $5 / 20 / 2015$ & $5 / 18 / 2015$ & $5 / 19 / 2015$ & -0.42 & $\$ 888.00$ & $\$ 27,840.15$ & $\$ 0.00$ & $\$ 2,083.30$ \\
\hline 82 & $5 / 20 / 2015$ & $5 / 19 / 2015$ & $5 / 20 / 2015$ & 0.71 & $\$ 882.00$ & $\$ 27,840.15$ & $\$ 7,083.50$ & $\$ 0.00$ \\
\hline 83 & $5 / 20 / 2015$ & $5 / 18 / 2015$ & $5 / 19 / 2015$ & -0.5 & $\$ 563.00$ & $\$ 16,800.19$ & $\$ 0.00$ & $\$ 2,499.95$ \\
\hline 84 & $6 / 23 / 2015$ & $5 / 22 / 2015$ & $6 / 2 / 2015$ & -20.29 & $\$ 546.00$ & $\$ 160,800.34$ & $\$ 0.00$ & $\$ 101,458.15$ \\
\hline 85 & $6 / 24 / 2015$ & $6 / 3 / 2015$ & $6 / 4 / 2015$ & -19.5 & $\$ 544.00$ & $\$ 16,800.19$ & $\$ 0.00$ & $\$ 97,499.95$ \\
\hline 86 & $6 / 25 / 2015$ & $6 / 4 / 2015$ & $6 / 4 / 2015$ & -20.42 & $\$ 569.00$ & $\$ 1,200.10$ & $\$ 0.00$ & $\$ 102,083.25$ \\
\hline 87 & $6 / 29 / 2015$ & $6 / 4 / 2015$ & $6 / 8 / 2015$ & -20.62 & $\$ 569.00$ & $\$ 54,600.05$ & $\$ 0.00$ & $\$ 103,124.90$ \\
\hline 88 & $7 / 12 / 2015$ & $6 / 12 / 2015$ & $6 / 22 / 2015$ & -19.5 & $\$ 4,436.00$ & $\$ 1,147,201.92$ & $\$ 0.00$ & $\$ 97,499.85$ \\
\hline 89 & $7 / 16 / 2015$ & $6 / 12 / 2015$ & $6 / 24 / 2015$ & -21.62 & $\$ 4,452.00$ & $\$ 1,358,402.69$ & $\$ 0.00$ & $\$ 108,124.70$ \\
\hline 90 & $6 / 13 / 2015$ & $5 / 18 / 2015$ & $5 / 27 / 2015$ & -16.5 & $\$ 547.00$ & $\$ 132,000.48$ & $\$ 0.00$ & $\$ 82,499.85$ \\
\hline 91 & $6 / 13 / 2015$ & $5 / 18 / 2015$ & $5 / 27 / 2015$ & -16.5 & $\$ 539.00$ & $\$ 132,000.48$ & $\$ 0.00$ & $\$ 82,499.85$ \\
\hline 92 & $6 / 16 / 2015$ & $5 / 27 / 2015$ & $6 / 1 / 2015$ & -14.5 & $\$ 545.00$ & $\$ 71,400.10$ & $\$ 0.00$ & $\$ 72,499.90$ \\
\hline 93 & $6 / 16 / 2015$ & $5 / 27 / 2015$ & $6 / 1 / 2015$ & -14.5 & $\$ 549.00$ & $\$ 71,400.10$ & $\$ 0.00$ & $\$ 72,499.90$ \\
\hline 94 & $6 / 7 / 2015$ & $5 / 18 / 2015$ & $5 / 20 / 2015$ & -17.29 & $\$ 50.00$ & $\$ 3,420.03$ & $\$ 0.00$ & $\$ 86,458.25$ \\
\hline 95 & $6 / 13 / 2015$ & $5 / 21 / 2015$ & $5 / 27 / 2015$ & -16.5 & $\$ 50.00$ & $\$ 8,880.02$ & $\$ 0.00$ & $\$ 82,499.90$ \\
\hline 96 & $6 / 16 / 2015$ & $5 / 27 / 2015$ & $6 / 1 / 2015$ & -14.5 & $\$ 50.00$ & $\$ 7,140.01$ & $\$ 0.00$ & $\$ 72,499.90$ \\
\hline 97 & $6 / 3 / 2015$ & $6 / 1 / 2015$ & $6 / 2 / 2015$ & -0.29 & $\$ 50.00$ & $\$ 1,680.00$ & $\$ 0.00$ & $\$ 1,458.25$ \\
\hline 98 & $6 / 6 / 2015$ & $6 / 3 / 2015$ & $6 / 4 / 2015$ & -1.5 & $\$ 50.00$ & $\$ 1,680.00$ & $\$ 0.00$ & $\$ 7,499.95$ \\
\hline 99 & $5 / 29 / 2015$ & $5 / 18 / 2015$ & $5 / 27 / 2015$ & -1.5 & $\$ 1,887.00$ & $\$ 448,801.63$ & $\$ 0.00$ & $\$ 7,499.85$ \\
\hline 100 & $6 / 1 / 2015$ & $5 / 27 / 2015$ & $6 / 1 / 2015$ & 0.5 & $\$ 1,869.00$ & $\$ 242,760.33$ & $\$ 5,000.20$ & $\$ 0.00$ \\
\hline 101 & $6 / 13 / 2015$ & $6 / 1 / 2015$ & $6 / 10 / 2015$ & -2.29 & $\$ 1,869.00$ & $\$ 448,801.14$ & $\$ 0.00$ & $\$ 11,458.15$ \\
\hline 102 & $6 / 19 / 2015$ & $6 / 11 / 2015$ & $6 / 17 / 2015$ & -1.5 & $\$ 1,869.00$ & $\$ 301,921.14$ & $\$ 0.00$ & $\$ 7,499.90$ \\
\hline 103 & $6 / 23 / 2015$ & $6 / 17 / 2015$ & $6 / 22 / 2015$ & -0.5 & $\$ 1,869.00$ & $\$ 242,760.33$ & $\$ 0.00$ & $\$ 2,499.90$ \\
\hline 104 & $5 / 24 / 2015$ & $5 / 18 / 2015$ & $5 / 22 / 2015$ & -1.5 & $\$ 53.00$ & $\$ 6,000.03$ & $\$ 0.00$ & $\$ 7,499.90$ \\
\hline 105 & $5 / 29 / 2015$ & $5 / 22 / 2015$ & $5 / 27 / 2015$ & -1.5 & $\$ 54.00$ & $\$ 7,140.01$ & $\$ 0.00$ & $\$ 7,499.90$ \\
\hline 106 & $5 / 18 / 2015$ & $5 / 18 / 2015$ & $5 / 18 / 2015$ & 0.38 & $\$ 51.00$ & $\$ 60.00$ & $\$ 3,750.00$ & $\$ 0.00$ \\
\hline 107 & $5 / 20 / 2015$ & $5 / 18 / 2015$ & $5 / 19 / 2015$ & -0.5 & $\$ 118.00$ & $\$ 3,360.04$ & $\$ 0.00$ & $\$ 2,499.95$ \\
\hline 108 & $6 / 1 / 2015$ & $5 / 18 / 2015$ & $6 / 1 / 2015$ & 0.5 & $\$ 113.00$ & $\$ 40,800.12$ & $\$ 5,000.40$ & $\$ 0.00$ \\
\hline 109 & $6 / 1 / 2015$ & $5 / 18 / 2015$ & $6 / 1 / 2015$ & 0.5 & $\$ 52.00$ & $\$ 20,400.06$ & $\$ 5,000.40$ & $\$ 0.00$ \\
\hline 110 & $6 / 1 / 2015$ & $5 / 18 / 2015$ & $6 / 1 / 2015$ & 0.5 & $\$ 276.00$ & $\$ 102,000.31$ & $\$ 5,000.40$ & $\$ 0.00$ \\
\hline 111 & $6 / 9 / 2015$ & $6 / 1 / 2015$ & $6 / 9 / 2015$ & 0.5 & $\$ 264.00$ & $\$ 57,300.12$ & $\$ 5,000.30$ & $\$ 0.00$ \\
\hline
\end{tabular}

